\title{
Thermodynamics of Polymer Blends
}

\author{
Evangelos Manias and Leszek A. Utracki
}

\section{Contents}

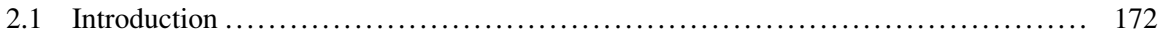

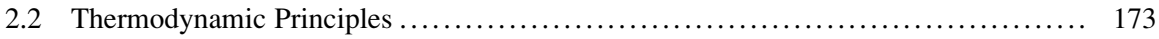

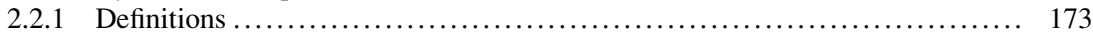

2.2.2 The Three Laws of Thermodynamics .............................. 175

2.2.3 Interrelations Between Thermodynamic Variables ...................... 177

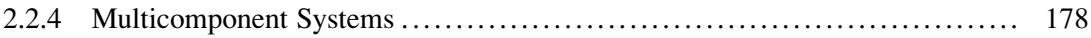

2.3 Thermodynamics of a Single-Component System .............................. 179

2.3.1 Equation of State (EoS) or PVT Relationships ...................... 179

2.3.2 Solid-Liquid and Vapor-Liquid Equilibria ............................. 187

2.3.3 Gibbs Phase Rule ............................................... 189

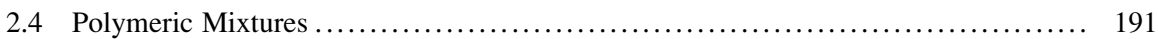

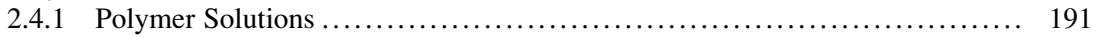

2.4.2 Polymer Blends: Definitions and Miscibility ........................... 193

2.5 Theories of Liquid Mixtures ............................................ 196

2.5.1 Lattice, Cell, and Hole Theories .................................. 196

2.5.2 Off-Lattice Theories ............................................. 209

2.5.3 Polymer Reference Interaction Site Model (PRISM) .................. 238

2.5.4 Summary of Theoretical Approaches .............................. 239

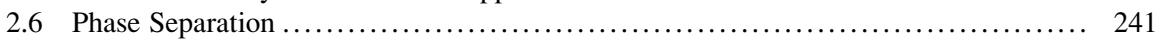

2.6.1 Thermodynamics of Phase Separation ............................. 241

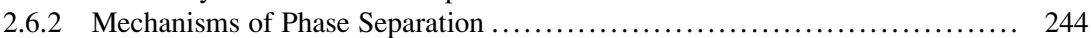

2.6.3 Phase Diagrams ............................................... 252

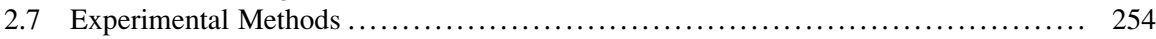

2.7.1 PVT and Related Measurements ............................... 255

2.7.2 Determination of Interaction Parameters ........................... 256

Leszek A. Utracki: deceased.

E. Manias $(\bowtie)$

Department of Materials Science and Engineering, Pennsylvania State University, University Park, PA, USA

e-mail:manias@psu.edu

L.A. Utracki

National Research Council Canada, Industrial Materials Institute, Boucherville, QC, Canada 


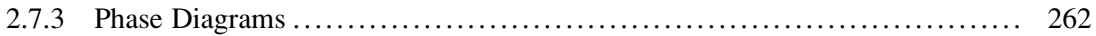

2.7.4 Indirect Methods for Polymer/Polymer Miscibility .................... 266

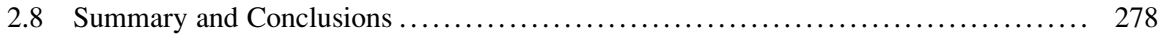

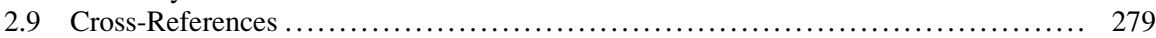

Notations and Abbreviations ................................................ 279

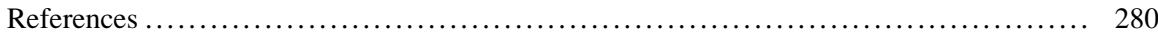

\section{Abstract}

This chapter summarizes the thermodynamics of multicomponent polymer systems, with special emphasis on polymer blends and mixtures. After a brief introduction of the relevant thermodynamic principles - laws of thermodynamics, definitions, and interrelations of thermodynamic variables and potentials - selected theories of liquid and polymer mixtures are provided: Specifically, both lattice theories (such as the Flory-Huggins model, Equation of State theories, and the gas-lattice models) and off-lattice theories (such as the strong interaction model, heat of mixing approaches, and solubility parameter models) are discussed and compared. Model parameters are also tabulated for the each theory for common or representative polymer blends. In the second half of this chapter, the thermodynamics of phase separation are discussed, and experimental methods - for determining phase diagrams or for quantifying the theoretical model parameters - are mentioned.

\subsection{Introduction}

Performance of polymer blends depends on the properties of polymeric components, as well as how they are arranged in space. The spatial arrangement is controlled by the thermodynamics and flow-imposed morphology. The word "thermodynamics" invariably brings to mind "miscibility." However, thermodynamics has a broader use for the practitioners of polymer science and technology than predicting miscibility. The aim of this chapter is to describe how to measure, interpret, and predict the thermodynamic properties of polymer blends, as well as where to find the relevant information and/or numerical values.

Determination of such thermodynamic properties as the phase diagram or the Flory-Huggins binary interaction parameter, $\chi_{12}$, is in principle difficult. The difficulties originate in high viscosity of macromolecular species, thus slow diffusion toward the equilibrium, heat generation when mixing, and thermal degradation at processing relevant temperatures.

For these reasons, there is a tendency to use values obtained from low molecular homologues or solutions. Furthermore, it is an accepted practice to purify the polymers before measuring their thermodynamic properties. However, most industrial polymers are modified by incorporating low molecular weight additives. Furthermore, they are processed under high flow rates and stresses that preclude the possibility of thermodynamic equilibrium. For these and other reasons, a direct application of the laboratory data to industrial systems may not always be appropriate or advisable. 
Another difficulty originates in the lack of theories able to predict variation of thermodynamic properties for commercially relevant systems with modifiers. Different additive compositions are used by different manufacturers of the same polymer. Some of these are even being "used up" during processing or during the products' lifetime, their content, and chemical structure change. They may significantly affect the thermodynamic properties of a polymeric mixture, by the physical, viz., that of a cosolvent, and the chemical effects. For example, additives of one polymeric component of a blend may chemically react with additives of another polymeric component, mutually neutralizing each other. In particular, these effects may be large as far as the surface and interface energies are concerned.

\subsection{Thermodynamic Principles}

\subsubsection{Definitions}

For convenience, thermodynamic systems are usually assumed closed, isolated from the surroundings. The laws that govern such systems are written in terms of two types of variables: intensive (or intrinsic) that do not depend on the mass and extensive that do. By definition, extensive variables are additive, that is, their value for the whole system is the sum of their values for the individual parts. For example, volume, entropy, and total energy of a system are extensive variables, but the specific volume (or its reciprocity - the density), molar volume, or molar free energy of mixing are intensive. It is advisable to use, whenever possible, intensive variables.

The main independent variables are the temperature $(T)$, pressure $(P)$, and composition (expressed as number of molecules, $N_{i}$, for each component molecule species, $i$, or through the respective molar fractions, $x_{i}$, or volume fractions, $\phi_{i}$ ). The principal thermodynamic terms are listed in Table 2.1, whereas Table 2.2 provides values of constants often used in thermodynamic calculations.

\subsubsection{Thermodynamic Potentials and the Concept of Free Energy}

In thermodynamics, any energy definition for a system is expressed in terms of pairs of thermodynamic variables, termed as conjugate variables. Conjugate pairs are $T$ and $S$ (with $T S$ relating to heat), $P$ and $V$ (with $P V$ related to mechanical work), $\mu$ and $N$, and so on. In each pair, one of the variables is an intensive variable (e.g., $T, P$, $\mu$, etc., that can be considered as a "generalized force"), and the other variable is extensive (e.g., $S, V, N$, respectively, that is considered as the respective "generalized displacement"). The construction of all energy expressions, i.e., thermodynamic potentials, is based on conjugate variables pairs, and all energy changes are associated with products of the absolute value of one conjugate variable multiplied by the change of the other from each pair, i.e., $T \Delta S, S \Delta T, P \Delta V, V \Delta P, \mu \Delta N$, and so on.

For every thermodynamic system, there is a finite number of $D$ conjugate pairs describing it (Alberty 2001), or, equivalently, $D$ independent variables are needed to describe the extensive state of the system (natural variables). $D$ is also called the "thermodynamic dimensionality" of the system, or, equivalently, the thermodynamic 
Table 2.1 Definition of principal thermodynamic terms

\begin{tabular}{|c|c|c|}
\hline Term & Symbol & Definition \\
\hline Internal energy & $U$ & $U=U(N, V, S)$ comprises of heat, $Q$, work, $W$ \\
\hline Entropy & $S$ & $\begin{array}{l}S=S(N, V, U) \text { with } S=k \ln \Omega ; \text { where } \Omega \text { is a measure of } \\
\text { the multiplicity of states }\end{array}$ \\
\hline Enthalpy & $H$ & $H=H(N, P, S)$ with $H=U+P V$ \\
\hline Helmholtz potential & $F$ & $F=F(N, V, T)$ with $F=U-T S=H-P V-T S$ \\
\hline Gibbs potential & $G$ & $G=G(N, P, T)$ with $G=U+P V-T S=H-T S$ \\
\hline Landau potential & $\Omega$ & $\Omega=\Omega(T, V, \mu)$ with $\Omega=U-T S-\mu N$ \\
\hline $\begin{array}{l}\text { Extensive properties of } \\
\text { a mixture }\end{array}$ & $Z_{m}$ & $Z_{m}$ can be $E_{m}$ or $S_{m}$ or $F_{m}$ or $H_{m}$, etc. \\
\hline $\begin{array}{l}\text { Change of an extensive } \\
\text { property due to mixing }\end{array}$ & $\Delta Z_{m}$ & $\Delta Z_{m}=Z_{m}-\Sigma x_{i} Z_{i}=\sum x_{i}\left(Z_{i}-Z_{i}^{0}\right)=\sum x_{i} \Delta Z_{i}$ \\
\hline Ideal solutions & $\Delta G^{I}$ & $\begin{array}{l}\Delta G^{I}=-T \Delta S^{I}=k T N \Sigma x_{i} \ln x_{i}, \text { thus no interactions, } \\
\Delta H^{I}=0, \text { and total randomness of molecular placement, } \\
\Delta V^{I}=0\end{array}$ \\
\hline Regular solutions & $\Delta G^{R}$ & $\begin{array}{l}\Delta G^{R}=\Delta H^{P}-T \Delta S^{I} ; \Delta H^{R} \propto \frac{1}{2}\left(\varepsilon_{11}+\varepsilon_{22}\right)-\varepsilon_{12} . \\
\text { The molecular interactions are nonspecific, without } \\
\text { associations, hydrogen or dipole-dipole bonding, } \\
\text { distribution, orientation, etc. }\end{array}$ \\
\hline Excess properties & $\Delta Z^{E}$ & $\Delta Z^{E}=\Delta Z-\Delta Z^{I}$ \\
\hline Athermal solutions & $\Delta G^{A}$ & $\Delta G^{A}=-T \Delta S ; \Delta H^{A}=0$ \\
\hline Combinatorial entropy & $\Delta S^{c o m b}$ & $\begin{array}{l}\text { That part that originates from the number of possible } \\
\text { placements of molecules in the lattice of an athermal } \\
\text { solution (the latter assumption usually is abandoned in the } \\
\text { following derivations) }\end{array}$ \\
\hline Equation of state (EoS) & $P V T$ & The relation between $\mathrm{P}, \mathrm{V}$, and $\mathrm{T}$ for a material \\
\hline Reducing variables & $\begin{array}{l}P^{*}, V^{*} \\
T^{*}\end{array}$ & $\begin{array}{l}\text { Reducing variables are characteristic materials' } \\
\text { parameters used to reduce the corresponding independent } \\
\text { variables, making them to follow respective } \\
\text { corresponding state equation(s) }\end{array}$ \\
\hline Corresponding states & $\tilde{P} \tilde{V} \tilde{T}$ & $\begin{array}{l}\text { Describing behavior of material by relations of its reduced } \\
\text { variables, e.g., } \tilde{P} \equiv P / P^{*}, \tilde{V} \equiv V / V^{*}, \tilde{T} \equiv T / T^{*}\end{array}$ \\
\hline Free volume & $V_{f}$ & $V_{f}=V-V_{o}$, where $V_{o} \simeq \lim _{T \rightarrow 0} V$ \\
\hline Solubility parameter & $\delta$ & $\begin{array}{l}\text { Can be calculated from the heat of vaporization } \Delta H^{V} \\
\left(\Delta H^{V}=R T^{2}(\partial \ln P / \partial T)_{\text {sat }}\right) \text { by } \delta=\sqrt{\left(\Delta H^{V}-R T\right) / V} \text { or } \\
\text { can also be calculated from the molecular structure }\end{array}$ \\
\hline
\end{tabular}

space of the system has $D$ dimensions. For example, in the simplest case of a singlephase ideal gas, there are three pairs of conjugate variables and, thus, $D=3$. By definition of the thermodynamic energy functions (thermodynamic potentials, cf. Table 2.1), this means that there will be $2^{D}$ unique thermodynamic potentials. The various thermodynamic potentials are defined considering as its independent variables one thermodynamic parameter from each conjugate pair; these are the $D$ natural variables of this thermodynamic potential (e.g., $U=U(N, V, S), F=F(N, V, T)$, $G=G(N, P, T)$, etc., see also Table 2.1). For the same example as above, the single-phase ideal gas, there will be $2^{3}=8$ thermodynamic potentials that can be 
Table 2.2 Useful constants

\begin{tabular}{lll}
\hline Constant & Symbol & Value \\
\hline Avogadro's number & $N_{A}$ & $6.02205 \times 10^{23}\left[\mathrm{~mol}^{-1}\right]$ \\
\hline Boltzmann constant & $k_{\mathrm{B}}$ & $1.38065 \times 10^{-23}\left[\mathrm{JK}^{-1}\right]$ \\
\hline Molar gas constant & $R=N_{A} k_{\mathrm{B}}$ & $8.31441\left[\mathrm{JK}^{-1} \mathrm{~mol}^{-1}\right]$ \\
\hline Molar volume at standard conditions & $V_{o}=R T_{o} / P_{o}$ & $22.41383 \times 10^{-3}\left[\mathrm{~m}^{3} \mathrm{~mol}^{-1}\right]$ \\
\hline Origin of Celsius scale & $T_{o}$ & $273.15[\mathrm{~K}]$ \\
\hline Planck's constant & $h$ & $6.62618 \times 10^{-34}[\mathrm{Js}]$ \\
\hline Standard atmospheric pressure & $P_{o}$ & $1.01325 \times 10^{5}\left[\mathrm{Nm}^{-2}\right]$ \\
\hline
\end{tabular}

defined to quantify its energy at a given state, or its energy change upon undergoing any arbitrary thermodynamic process. All $2^{D}$ thermodynamic potentials can be calculated for any system, and its corresponding differential can be calculated for any arbitrary process to quantify the corresponding energy change for this process (Alberty 2001). However, only one of these $2^{D}$ energy definitions adopts appropriate values and has appropriate behaviors/changes for each thermodynamic process, and this energy (thermodynamic potential) is the free energy of the system for this thermodynamic process. For a system undergoing a thermodynamic process, by definition:

1. The free energy adopts a minimum value at equilibrium.

2. The free energy change is negative for spontaneous processes.

Both the above are satisfied by a thermodynamic potential, when the thermodynamic process happens under conditions where its natural variables remain constant. For example, for a closed system $(N=$ constant $)$ undergoing a process under controlled temperature and volume ( $T, V$ are also constant), the Helmholtz potential $F=F(N, V, T)$ is the corresponding free energy; similarly, for the same system undergoing a process where $N P T$ are constant, the Gibbs potential $G=G(N, P, T)$ is now the free energy in this case; and so on.

New energy terms need to be added in the form of conjugate pairs when the system has additional energy contributions: e.g., if there exist more than one species of molecules, a separate $\mu_{i} N_{i}$ term is needed for each species $i$; if there are magnetic dipoles or spins, a $B M$ term is needed ( $B$ is the magnetic field, $M$ the system magnetization, and $B d M$ would be the associated energy change contribution in $d U$ ); and so on. Also, there may be additional mechanical work terms (beyond $P d V$ ), such as work associated with interfaces, $\gamma d A_{\text {inter }}$; work associated with mechanical deformation, $V \sum_{i j} \tau_{i j} d \varepsilon_{i j}$; or work associated for uniaxial deformation, $F d l$.

\subsubsection{The Three Laws of Thermodynamics}

Thermodynamics focuses on the state of material, usually in a closed system. Historically, the laws were empirically formulated using the well-accepted process of hypothesis, observation, and analysis. However, since the energetic state of a closed system is a sum of all the molecular and atomic motions, the statistical physics re-derived these laws from the first principles (Waldram 1985; Gupta 1990). 


\subsubsection{The First Law (Conservation of Energy)}

In a closed system, the total energy remains constant (also known as sine perpetuum mobile). Thus, for a closed system, the change of internal energy $(d U)$ :

$$
d U=d W+d Q=0
$$

where $d W$ represents the work done on or by the system and $d Q$ represents the change of the thermal energy content. Note that there are several forms of energy that can be classified as "work," for example, compression, friction, electromagnetic interaction, etc.

For a perfect gas at constant pressure, $P=$ const., Eq. 2.1 gives the relationship between the heat capacities at constant pressure and at constant volume, viz., $C_{P}-C_{V}=R$ ( $R$ is the gas constant). Similarly, at $T=$ const., Eq. 2.1 predicts that the external work can only be performed at a cost of the internal energy: $P d V=-d U$.

\subsubsection{The Second Law (The Principle of Entropy Increase)}

The energy always flows from a higher to a lower level; hence, the system tends to move toward a more uniform distribution of the local energetic states. Since the entropy provides a measure of randomness, in a closed system, the entropy never decreases:

$$
d S=\frac{d Q}{T} \geq 0
$$

The second law of thermodynamics is also known as the Carnot cycle principle that specifies that "heat can never pass from a colder to a warmer body without some other changes, connected therewith, occurring at the same time" (Clausius).

Equation 2.2 can also be written as:

$$
\begin{aligned}
& \left(\frac{\partial S}{\partial V}\right)_{T}=\frac{1}{T}\left(\frac{\partial Q}{\partial V}\right)_{T}=\left(\frac{\partial P}{\partial T}\right)_{V} \\
& \left(\frac{\partial S}{\partial P}\right)_{V}=\frac{1}{T}\left(\frac{\partial Q}{\partial P}\right)_{V}=\left(\frac{\partial V}{\partial T}\right)_{S}
\end{aligned}
$$

\subsubsection{The Third Law (Entropy Vanishes at Absolute Zero)}

In 1906, Nernst showed (experimentally) that the equilibrium entropy tends toward zero as the absolute temperature approaches zero:

$$
\begin{aligned}
\lim _{T \rightarrow 0} S & =\lim _{T \rightarrow 0} d S=0, \text { or equivalently } \\
S & =\int_{0}^{T} \frac{d Q_{\text {rev }}}{T}\left(\text { assuming } S_{T=0} \stackrel{\text { emp }}{=} 0\right)
\end{aligned}
$$

As a corollary, near zero $\mathrm{K}$ the change of entropy in any process is negligibly small. Thus, the third law is empirical, but so far no contradictory observation was reported. As a consequence of Eq. 2.3, near the absolute zero the coefficient of thermal expansion and the pressure coefficient are expected to vanish: 


$$
\begin{aligned}
& \lim _{T \rightarrow 0}\left(\frac{\partial S}{\partial P}\right)_{T}=\lim _{T \rightarrow 0}\left(\frac{\partial S}{\partial V}\right)_{T}=0 \\
& \lim _{T \rightarrow 0}\left(\frac{\partial V}{\partial T}\right)_{P}=-\lim _{T \rightarrow 0}\left(\frac{\partial P}{\partial T}\right)_{V}=0
\end{aligned}
$$

In summary, the first law is simply a statement of the law of conservation of energy, the second law is concerned only with differences in the entropy between (two) states, and the third law allows for the calculation of the absolute entropy of a state (assuming knowledge of the system's entropy at the absolute zero of temperature, that is at $T=0 \mathrm{~K}$ ).

\subsubsection{Interrelations Between Thermodynamic Variables}

The full differentials (or the total changes) of the principal thermodynamic potentials for a closed system $(N=$ const. $)$ cast in terms of their natural variables are

$$
\begin{array}{rr}
d F= & -S d T-P d V \\
d H= & T d S+V d P \\
d G= & -S d T+V d P
\end{array}
$$

Note that Eq. 2.4 implies that $F=F(T, V), H=H(S, P)$, and $G=G(T, P)$ and that the thermodynamic definitions of pressure, $P$, and temperature, $T$, are

$$
\begin{aligned}
P & =\frac{(\partial S / \partial V)_{U}}{(\partial S / \partial U)_{V}}=\left(\frac{\partial F}{\partial V}\right)_{T} \\
T & =\left(\frac{\partial H}{\partial S}\right)_{P}=\left(\frac{\partial U}{\partial S}\right)_{V}
\end{aligned}
$$

These relations are important when deriving an equation of state by statistical methods. In addition, they can naturally lead to another set of useful identities through the derivation of the mixed second derivatives of the thermodynamic potentials (known as Maxwell relations):

$$
\begin{aligned}
\frac{\partial^{2} U}{\partial S \partial V} & =+\left(\frac{\partial T}{\partial V}\right)_{S}=-\left(\frac{\partial P}{\partial S}\right)_{V} \\
\frac{\partial^{2} H}{\partial S \partial P} & =+\left(\frac{\partial T}{\partial P}\right)_{S}=+\left(\frac{\partial V}{\partial S}\right)_{P} \\
\frac{\partial^{2} F}{\partial T \partial V} & =-\left(\frac{\partial S}{\partial V}\right)_{T}=-\left(\frac{\partial P}{\partial T}\right)_{V} \\
\frac{\partial^{2} G}{\partial T \partial P} & =-\left(\frac{\partial S}{\partial P}\right)_{T}=+\left(\frac{\partial V}{\partial T}\right)_{P}
\end{aligned}
$$

Whereas, since the heat capacity $C$ is $\delta Q \equiv C \delta T$, using the second law of thermodynamics $(\delta S=\delta Q / T$, or $\delta Q=T \delta S)$ and Eq. 2.4 the heat capacities at 
constant pressure (or at constant volume) can directly connect the enthalpy and entropy gradients between two temperatures, for example,

$$
C_{P} \equiv T\left(\frac{\partial S}{\partial T}\right)_{P}=-\left(\frac{\partial H}{\partial T}\right)_{P} \quad \text { and } \quad C_{V} \equiv T\left(\frac{\partial S}{\partial T}\right)_{V}=-\left(\frac{\partial H}{\partial T}\right)_{V}
$$

Based on the Maxwell relations, the definitions of the isothermal compressibility $\left(K_{T}\right.$ or $\left.\beta_{T}\right)$, the isoentropic compressibility $\left(K_{S}\right.$ or $\left.\beta_{T}\right)$, and the thermal expansion coefficient $(\alpha)$ can be written with the $\ln V$ removed as, respectively,

$$
\begin{aligned}
K_{T} \text { or } \beta_{T} & \equiv\left(\frac{\partial P}{\partial \ln V}\right)_{T}=-\frac{1}{V}\left(\frac{\partial V}{\partial P}\right)_{T} \\
K_{S} \text { or } \beta_{S} & \equiv\left(\frac{\partial P}{\partial \ln V}\right)_{S}=-\frac{1}{V}\left(\frac{\partial V}{\partial P}\right)_{S} \\
\alpha & \equiv\left(\frac{\partial \ln V}{\partial T}\right)_{P}=+\frac{1}{V}\left(\frac{\partial V}{\partial T}\right)_{P}
\end{aligned}
$$

The parameter $K_{T}$ is also known as the isothermal bulk modulus and $\alpha$ as the volume expansion coefficient; these are interrelated by means of the Grüneisen constant, $\gamma$ :

$$
\gamma=-\frac{V}{C_{V}} \frac{(\partial V / \partial T)_{P}}{(\partial V / \partial P)_{T}}=\frac{\alpha V}{C_{V} \beta_{T}}
$$

Similarly, through the definition of isothermal and isoentropic compressibility, the ratio and difference of $C_{P}$ and $C_{V}$ can also be simplified to

$$
\begin{aligned}
& \frac{C_{P}}{C_{V}}=\frac{(\partial P / \partial V)_{S}}{(\partial P / \partial V)_{T}}=\frac{\beta_{T}}{\beta_{S}} \\
& C_{P}-C_{V}=T\left(\frac{\partial S}{\partial T}\right)_{P}-T\left(\frac{\partial S}{\partial T}\right)_{V}=V T \frac{\alpha^{2}}{\beta_{T}}
\end{aligned}
$$

\subsubsection{Multicomponent Systems}

In a multicomponent, closed system comprising $N_{i}$ moles of component $i$, the changes $d X$ in an extensive function of state $X$ (e.g., $U, F, G, S, H, C_{P}, C_{V}$, etc.) caused by the variation of composition are given by

$$
\begin{aligned}
& d X=\sum_{i} X_{i} d N_{i} \\
& \text { defining } X_{i} \equiv\left(\frac{\partial X}{\partial N_{i}}\right)_{N_{j \neq i}} \text { and, thus: } X=\sum_{i} X_{i} N_{i}
\end{aligned}
$$

where the functions $X_{i}$ are known as partial molar quantities. Thus, the full differentials of the state functions (viz., Eq. 2.4) can be expressed as 


$$
\begin{aligned}
d U & =T d S-P d V+\sum_{i} \mu_{i} d N_{i} \\
d F & =-S d T-P d V+\sum_{i} \mu_{i} d N_{i} \\
d H & =T d S+V d P+\sum_{i} \mu_{i} d N_{i} \\
d G & =-S d T+V d P+\sum_{i} \mu_{i} d N_{i}
\end{aligned}
$$

where $\mu_{\mathrm{i}}=\partial G / \partial \mathrm{N}_{\mathrm{i}}$ is the chemical potential of component $i$ and $N_{i}$ being a natural variable for all four above state functions, that is, $U=U(S, V, N), F=F(T, V, N)$, $H=H(S, P, N)$, and $G=G(T, P, N)$. The relationships in Eq. 2.11 indicate that in a closed multicomponent system $\left(N_{i}=\right.$ const.) any change of the independent variables must be reflected in a change of the chemical potentials $\left(\mu_{i}\right)$ :

$$
-S d T+V d P-\sum_{i} N_{i} d \mu_{i}=0
$$

Equation 2.12 is known as the Gibbs-Duhem relationship and is a depiction that the free energy of this grand canonical ensemble remains unchanged.

\subsection{Thermodynamics of a Single-Component System}

\subsubsection{Equation of State (EoS) or PVT Relationships}

\subsubsection{Equation of State (EoS)}

All theoretical equations of state suggest a corresponding state behavior of PVT properties that requires three scaling parameters $\left(P^{*}, V^{*}\right.$, and $\left.T^{*}\right)$. These define the corresponding state and are used to scale/reduce the $P V T$ toward $\tilde{P} \equiv P / P^{*}, \tilde{V} \equiv V / V^{*}, \tilde{T} \equiv T / T^{*}$. In his Ph.D. thesis of 1873 , van der Waals proposed the first EoS formulated in terms of corresponding state (reduced) variables. The relation can be written in terms of $P V T$ or in terms of reduced variables $\tilde{P} \tilde{V} \tilde{T}$, indicating expected observance of the corresponding state principle:

$$
\begin{aligned}
P & =\frac{R T}{V-b}-\frac{a}{V^{2}} \\
\frac{\tilde{P}}{\tilde{T}} & =\frac{8}{3 \tilde{V}-1}-\frac{3}{\tilde{T} \tilde{V}^{2}}
\end{aligned}
$$

$$
\begin{aligned}
& \left(\text { where } \tilde{P} \equiv P / P^{*}, \tilde{V} \equiv V / V^{*}, \tilde{T} \equiv T / T^{*},\right. \\
& \text { with } \left.V^{*}=3 b ; P^{*}=a / 27 b^{2} ; T^{*}=8 a / 27 R b\right)
\end{aligned}
$$

This approach demonstrated that the $V^{*}$ and $P^{*}$ are related to the van der Waals excluded volume $(b)$ and the cohesive energy density $(\propto a)$ of a system, a fact that has been largely used thereafter in subsequent EoS theories. Equation 2.13 also introduced the free volume concept; note that as $T \rightarrow 0, V \rightarrow b$. van der Waals 
considered that molecules move in "cells" defined by the surrounding molecules with a uniform potential. Furthermore, Eq. 2.13 allows, through the definition of the critical point $\left(P_{c}, T_{c}\right)$ in the $P-T$ phase diagram (vide infra), to connect $a$ and $b$ to the critical values of pressure and temperature, $P_{c}$ and $T_{c}$, which can be found tabulated from experimental data for various systems (viz., $a=27 R^{2} T_{c}^{2} / 64 P_{c}$, and $\left.b=R T_{c} / 8 P_{c}\right)$. This enables improved predictive capability over the ideal gas EoS. Finally, van der Walls also proposed a method to extend the single-component EoS of Eq. 2.13 to multicomponent mixtures by using the same relation with weight-averaged values of $a_{m}, b_{m}$ of the mixture calculated based on the mole compositions $\left(y_{i}\right)$ and the single-component $a_{i}, b_{i}$ of each component $i: a_{m}=\sum_{i} \sum_{j} y_{i} y_{j} \sqrt{a_{i} a_{j}}$ and $b_{m}=\sum_{i} y_{i} b_{i}$. Thus, although the van der Walls EoS is not used today for any practical purpose, it is purely pedagogical, it does clearly demonstrate the corresponding state principle, it predicts continuity of matter between gas and liquid phases, it provides a mixture rule for EoS application in multicomponent mixtures, and it laid the foundations for modern EoS theories.

The volume, within which the center of a molecule can freely move, is what defines its free volume (or, more accurately, free volume relates to the excess empty volume beyond the per-molecule unoccupied volume). Thus, one may distinguish and define:

1. Total volume, $V$.

2. Occupied volume, $V_{o}$ (usually defined as the $V$ at $T=0 \mathrm{~K}$ ).

3. Free volume, $V_{f}=V-V_{o}$.

4. Doolittle's free volume fraction, $f_{D}=V_{f} / V_{o}$.

5. Free volume fraction, $f=V_{f} / V=1 /\left(1+1 / f_{D}\right)$ (to be used here).

Detailed methods of computation of the van der Waals excluded volume (for any chemical structure) have been developed (van Krevelen 1976). Thermodynamically, the free volume is expressed in terms of the entropy of vaporization:

$$
\begin{gathered}
P=\frac{R T}{V_{f}} \exp \left(-\frac{\Delta H^{V}}{R T}\right)=\frac{R T}{V_{f}} \exp \left(-\frac{\Delta S^{V}}{R}\right)=\frac{R T}{V} \\
\text { or }: \Delta S^{V}=R \ln \left(V / V_{f}\right) \text { and } \Delta H^{V}=T^{V} \Delta S^{V}
\end{gathered}
$$

Over the years, many versions of the EoS theories have been proposed (see, e.g., Table 2.3). Several comprehensive reviews of the EoS used in polymer thermodynamics have been published. For example, one review (Curro 1974) discussed applications of EoS within a full range of materials and variables, viz., to crystals, glasses, molten polymers, and monatomic liquids. This review discusses fundamentals of the theories and it provides a list of available experimental data. The comparison between different EoS was made on two levels, first by comparing the derived expressions for physical quantities (e.g., the characteristic reducing parameters, cohesive energy density, or internal pressure) and then comparing how well the EoS describes the observed PVT dependencies for polymers. A second type of reviews focused on summarizing PVT parameters for the molten state of polymers (Zoller 1989; Rodgers 1993a, b; Cho 1999). The authors here examined and compared several EoS theories, e.g., Spencer and Gilmore (1949) (S-G), 
Table 2.3 A summary of a few EoS, mentioned in this section

\section{Cell models}

Flory-Orwoll-Vrij (FOV)

$\frac{\tilde{P} \tilde{V}}{\tilde{T}}=\frac{\tilde{V}^{1 / 3}}{\left(\tilde{V}^{1 / 3}-1\right)}-\frac{1}{\tilde{T} \tilde{V}} \quad \tilde{P} \equiv \frac{P}{P^{*}}, \tilde{V} \equiv \frac{V}{V^{*}}, \tilde{T} \equiv \frac{T}{T^{*}} \quad$ Eq. 2.15

Prigogine $(\mathrm{P})$

$\overline{\tilde{P} \tilde{V}} \frac{\tilde{V}^{1 / 3}}{\tilde{T}}-\frac{2}{\tilde{T}}\left(\frac{1.2045}{\tilde{V}^{1 / 3}-2^{-1 / 6}}-\frac{1.011}{\tilde{V}^{4}}\right) \quad \tilde{P} \equiv \frac{P}{P^{*}}, \tilde{V} \equiv \frac{V}{V^{*}}, \tilde{T} \equiv \frac{T}{T^{*}} \quad$ Eq. 2.17

Dee and Walsh (D-W)

$\frac{\tilde{P} \tilde{V}}{\tilde{T}}=\frac{\tilde{V}^{1 / 3}}{\left(\tilde{V}^{1 / 3}-2^{-1 / 6} q\right)}-\frac{2}{\tilde{T}}\left(\frac{1.2045}{\tilde{V}^{2}}-\frac{1.011}{\tilde{V}^{4}}\right) \quad \tilde{P} \equiv \frac{P}{P^{*}}, \tilde{V} \equiv \frac{V}{V^{*}}, \tilde{T} \equiv \frac{T}{T^{*}} \quad q=1.07$

Simha and Somcynsky (S-S) hole model

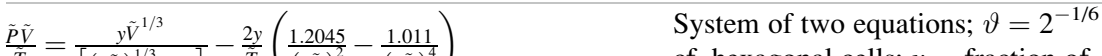

$\begin{array}{ll}\frac{\tilde{P} \tilde{V}}{\tilde{T}}=\frac{y \tilde{V}}{\left[(y \tilde{V})^{1 / 3}-\vartheta y\right]}-\frac{2 y}{\tilde{T}}\left(\frac{1.2045}{(y \tilde{V})^{2}}-\frac{1.011}{(y \tilde{V})^{4}}\right) & \text { cf. hexagonal cells; } y=\text { fraction of } \\ \frac{s}{3 c}\left[1+\frac{\ln (1-y)}{y}\right]=\frac{\frac{1}{3}(y \tilde{V})^{1 / 3}-\vartheta y}{(y \tilde{V})^{1 / 3}-\vartheta y}-\frac{y}{6 \tilde{T}}\left(\frac{2.409}{(y \tilde{V})^{2}}-\frac{3.033}{(y \tilde{V})^{4}}\right) & \text { occupied sites; } s / 3 c \simeq 1 \text { for polymers } \\ & \tilde{P} \equiv \frac{P}{P^{*}}, \tilde{V} \equiv \frac{V}{V^{*}}, \tilde{T} \equiv \frac{T}{T^{*}} \quad \text { Eq. } 2.19\end{array}$

Eq. 2.19

\section{Lattice fluid models}

Sanchez and Lacombe (S-L)

$\frac{\tilde{P} \tilde{V}}{\tilde{T}}=-\tilde{V}\left[\ln \left(1-\frac{1}{\tilde{V}}\right)-\frac{1}{\tilde{V}}\right]-\frac{1}{\tilde{T} \tilde{V}} \quad \tilde{P} \equiv \frac{P}{P^{*}}, \tilde{V} \equiv \frac{V}{V^{*}}, \tilde{T} \equiv \frac{T}{T^{*}} \quad$ Eq. 2.16

Jung

$\frac{\tilde{P} \tilde{V}}{\tilde{T}}=-\tilde{V}\left[\ln \left(1-\frac{1}{\tilde{V}}\right)+\frac{1}{\tilde{V}}\right]-\frac{4}{\tilde{T}}\left(\frac{1.2045}{\tilde{V}^{2}}-\frac{1.011}{\tilde{V}^{4}}\right) \quad \tilde{P} \equiv \frac{P}{P^{*}}, \tilde{V} \equiv \frac{V}{V^{*}}, \tilde{T} \equiv \frac{T}{T^{*}} \quad$ Eq. 2.18

\section{Empirical PVT relations}

Tait equation (4-parameter)

$\begin{array}{ll}V(P, T)=V(0, T)\left[1-C \ln \left(1+\frac{P}{B(T)}\right)\right] & \text { with } C=0.0894 ; V \\ & (0, T)=V_{o} e^{\alpha T} ;\end{array}$

Eq. 2.22

$B(T)=B_{o} e^{-B_{1} T}$

Hartmann and Haque (H-H)

$\tilde{P} \tilde{V}^{5}=\tilde{T}^{3 / 2}-\ln \tilde{V}$

$P^{*}=B_{o}, V^{*}=V_{o}, T^{*}=T_{o}$

Eq. 2.23

Sanchez and Cho (S-C)

$\tilde{V}=\frac{1}{1-\tilde{T}} \exp \left[\frac{\omega}{(1-\omega) B_{1}}\left\{1-\left(1+\frac{B_{1} \tilde{P} \exp (9 \tilde{T})}{\omega}\right)^{1-\omega}\right\}\right] \quad B_{1}=10.2 ; \omega=0.9$

Eq. 2.24

Flory et al. (1964) (FOV), Sanchez-Lacombe (1976, 1977, 1978) (S-L), Simha and Somcynsky (1969) (S-S), Prigogine et al. (1953, 1957) (P), Dee and Walsh (1988) (D-W), Hartmann and Haque (1985) (H-H), and Sanchez and Cho (1995) (S-C), and tabulated the respective corresponding state values $\left(P^{*}, V^{*}\right.$, and $\left.T^{*}\right)$ for most common polymers. These comparisons span across the different types of EoS models, from cell models (FOV, P, D-W), to lattice-fluid (S-L) and hole (S-S) models, to semiempirical approaches (H-H, S-C), comparing the validity of distinctly different EoS approaches across large numbers of different homopolymers and copolymers. All reviews seem to build a consensus on the comparative accuracy of the various EoS: Zoller (1989) reported large deviations $(\leq 0.01 \mathrm{~mL} / \mathrm{g})$ 
for $\mathrm{S}-\mathrm{G}$, the FOV and $\mathrm{S}-\mathrm{L}$ were useful only at low $P$ and over small $P$ ranges, whereas S-S and S-C consistently provided the best representation of data over extended ranges of $T$ and $P$, with average deviations in volume of $\leq 0.003 \mathrm{~mL} / \mathrm{g}$ $(\mathrm{S}-\mathrm{S})$ and $\leq 0.0004 \mathrm{~mL} / \mathrm{g}(\mathrm{S}-\mathrm{C})$, compared to the experimental values. Rodgers (1993a, b) and Cho (1999) reach similar comparative conclusions, based on additional experimental $P V T$ data, reporting that the D-H modified cell, the $\mathrm{S}-\mathrm{S}$ hole, the $\mathrm{P}$ cell models, and the semiempirical $\mathrm{H}-\mathrm{H}$ and $\mathrm{S}-\mathrm{C}$ models, were all found to provide good fits of polymer liquid PVT data over the full range of experimental pressures, whereas the FOV and the S-L EoS were both significantly less accurate when applied over wide pressure ranges.

The FOV model can be summarized as

$$
\begin{aligned}
& \frac{\tilde{P}}{\tilde{T}}=\frac{1}{\tilde{V}^{2 / 3}\left(\tilde{V}^{1 / 3}-1\right)}-\frac{1}{\tilde{T}} \cdot \frac{1}{\tilde{V}^{2}} \\
& \text { with: } \quad V^{*}=\rho_{*}^{3} ; \quad P^{*}=c k_{B} T^{*} / V^{*} ; \quad T^{*}=s_{*} \eta_{*} /\left(2 c V^{*} k_{B}\right)
\end{aligned}
$$

where $\rho_{*}$ is the "hard-sphere" radius, $s *$ the number of contacts per segment, $\eta_{*}$ the segment-segment interaction energy, and $c$ the coordination number $\left(k_{B}\right.$ is the Boltzmann constant).

The S-L model, better known as the "lattice-fluid model," introduces vacancies into the classical incompressible Flory-Huggins model (vide infra). The lattice vacancy is treated as a pseudoparticle in the system. This model can be summarized as

$$
\frac{\tilde{P}}{\tilde{T}}=-\left[\ln \left(1-\frac{1}{\tilde{V}}\right)+\frac{1}{\tilde{V}}\right]-\frac{1}{\tilde{T}} \cdot \frac{1}{\tilde{V}^{2}}
$$

with $V^{*}=M_{W} / r \rho^{*}$ (where $M_{W}$ is the weight-averaged molecular weight, while $\rho^{*}$ is the characteristic density parameter, $\left.\rho^{*}=1 / V^{*}\right), P^{*}=r N_{1} \varepsilon^{*} / V^{*}$, and $T^{*}=\varepsilon^{*} / k_{\mathrm{B}}$ (where $\varepsilon^{*}$ is the van der Walls interaction energy). The parameter $r$ represents the number of lattice sites occupied by the $r$-mer - its presence in the EoS negates the principle of corresponding states. The latter can be recovered only for $r \rightarrow \infty$.

The Prigogine simple cell model $(\mathrm{P})$ considers each monomer in the system to be trapped in the cell created by its surroundings. The general cell potential, generated by the surroundings, is simplified to be athermal (cf. free volume theory), whereas the mean potential between the centers of different cells are described by the Lennard-Jones 6-12 potential. The $\mathrm{P}$ model EoS can be summarized as

$$
\frac{\tilde{P}}{\tilde{T}}=\frac{1}{\tilde{V}^{2 / 3}\left(\tilde{V}^{1 / 3}-2^{-1 / 6}\right)}-\frac{2}{\tilde{T}} \cdot\left(\frac{1.2045}{\tilde{V}^{3}}-\frac{1.011}{\tilde{V}^{5}}\right)
$$

where the factor $2^{-1 / 6}$ originates from the hexagonal close packing lattice used as a cell geometry and the factors 1.2045 and 1.011 correct the effects of higher coordination shells on the internal energy. Another, largely unnoticed, 
EoS was proposed (Jung 1996), by employing a continuous lattice and using a Lennard-Jones 6-12 potential to modify the S-L simple lattice fluid. This method combines elements from both S-L and P models, and the obtained EoS can be summarized as

$$
\frac{\tilde{P}}{\tilde{T}}=-\left[\ln \left(1-\frac{1}{\tilde{V}}\right)+\frac{1}{\tilde{V}}\right]+\frac{4}{\tilde{T}} \cdot\left(\frac{1.2045}{\tilde{V}^{3}}-\frac{1.011}{\tilde{V}^{5}}\right)
$$

with the same definitions as the S-L simple lattice-fluid EoS. To examine the ability of this EoS to describe PVT dependencies, the author used experimental data of eight polymers and compared with the FOV, S-L, S-S, and D-W relations. The evaluation was performed computing errors in describing the volume $(\Delta V)$, as well as thermal expansivity and isothermal compressibility. As in the previous evaluations, S-S dependence performed the best. For the description of $P V T$, the new EoS performed as well as that of D-W, but for the expansivity and compressibility, it outperformed the latter EoS.

The S-S EoS derived by Simha and Somcynsky (1969) is based on the Prigogine cell model by introducing lattice imperfections (holes, unoccupied sites). In S-S, a liquid is represented as a mixture of $y$ occupied and $h(=1-y)$ unoccupied sites; thus, following the "Significant Liquid Structures" nomenclature (Eyring and Jhon 1969), the model considers a liquid as being an intermediate between solid and gas. To derive the EoS, the authors first calculated the partition function, $Z$, for all possible number of arrangements of occupied sites and empty holes in a lattice with $z$ coordination number. The Helmholtz free energy is directly given, $F=-k_{B} T \ln Z$, and its differentiation gives the pressure and, thus, the equation of state. Minimization of the Helmholtz free energy $F$ provides a second relation that must be solved simultaneously with the EoS:

$$
\begin{aligned}
& \frac{\tilde{P}}{\tilde{T}}=\frac{y}{\tilde{V}^{2 / 3}\left[(y \tilde{V})^{1 / 3}-2^{-1 / 6} y\right]}-\frac{2 y^{2}}{\tilde{T}} \cdot\left(\frac{1.2045}{(y \tilde{V})^{3}}-\frac{1.011}{(y \tilde{V})^{5}}\right) \\
& \frac{s}{3 c}\left[1+\frac{\ln (1-y)}{y}\right]=\frac{\frac{1}{3}(y \tilde{V})^{1 / 3}-2^{-1 / 6} y}{(y \tilde{V})^{1 / 3}-2^{-1 / 6} y}-\frac{y}{6 \tilde{T}}\left(\frac{2.409}{(y \tilde{V})^{2}}-\frac{3.033}{(y \tilde{V})^{4}}\right)
\end{aligned}
$$

with : $y=$ fraction of occupied sites;

$s / 3 c \simeq 1$ in normal practice for polymers

Of all the EoS used for polymeric species, the one derived by $\mathrm{S}-\mathrm{S}$ was the first to explicitly consider the hole fraction, $h=1-y$. Equation 2.19 provides a corresponding state description of $P V T$ behavior of any liquid. Once the four characteristic parameters: $P^{*}, V^{*}, T^{*}$, and $3 c / s$ are known, the specific volume and all its derivatives are known in the full range of $P$ and $T$. For linear polymers, where $3 c / s \simeq 1$, only the three usual parameters $\left(P^{*}, V^{*}\right.$, and $\left.T^{*}\right)$ are required. Values of 
$P^{*}, V^{*}$, and $T^{*}$ for selected polymers are listed in Table 2.4. At atmospheric pressure, $\tilde{P}=0$, and within the range of reduced volumes, $0.95<\tilde{V} 1.40$, Eq. 2.19 predicts that the volume expansion with $T$ should follow the dependence (Simha and Weil 1970):

$$
\ln \tilde{V}_{i}=S_{1}\left(s_{i}, c_{i}\right)+S_{2}\left(s_{i}, c_{i}\right) \tilde{T}_{i}^{3 / 2}
$$

\subsubsection{Frozen Free Volume Fraction}

The S-S EoS theory defined through Eq. 2.19 is valid for any liquid. However, upon cooling when the temperature reaches the glass transition region, part of the free volume fraction is no longer accessible for the molecular motion. The $\mathrm{S}-\mathrm{S}$ theory can also be used in this glassy region, if it can be estimated what part of the free volume is frozen as $T$ approaches the glass transition temperature, $T_{g}$. Experimentally, the frozen fraction of the free volume, $F F$, depends on the absolute value of $T_{g}$. This finding was first reported for several polymers at ambient pressure (Simha and Wilson 1973). Subsequently, the generality of this observation was confirmed by analyzing isobaric thermal expansion of PS for a wide range of pressures (Utracki and Simha 1997).

As shown in Fig. 2.1, the frozen fraction of the free volume, $F F$, follows the same dependence whether $T_{g}$ changes are caused by the polymeric chemical structure or by imposed pressure. The observed, general dependence follows the empirical relation:

$$
F F=0.997-4.75 \times 10^{-4} T_{g}-1.52 \times 10^{-6} T_{g}^{2} \quad\left(R_{\mathrm{fit}}=0.975\right)
$$

It is gratifying to see that as the glass transition temperature approaches the absolute zero, $T_{g} \rightarrow 0 \mathrm{~K}$, Eq. 2.21 predicts that all free volume should freeze, $F F\left(T_{g}=0 \mathrm{~K}\right)=1$. On the other hand, at the high-temperature range, as $T_{g}$ exceeds $669 \mathrm{~K}\left(396^{\circ} \mathrm{C}\right)$, all free volume should be accessible to thermal motion in the glassy state, i.e., $F F\left(T_{g} \geq 400{ }^{\circ} \mathrm{C}\right)=0$. Thus, it is to be expected that polymers at high temperature will have the same thermal expansion coefficient across the glass transition temperature, viz., $\alpha_{L}=\alpha_{G}$, where subscripts $L$ and $G$ indicate liquid and glassy state, respectively.

\subsubsection{Empirical PVT Relations}

Starting from a different viewpoint, one can reach empirically justified EoS by phenomenological arguments used to build universal behaviors (functions) and fit them to experimental $P V T$ data. The most common such approach for polymers is an isothermal compressibility $V$ - $P$ model (Tait 1888) known as the Tait equation:

$$
\begin{aligned}
& V(P, T)=V(0, T)[1-C \ln (1+P / B(T))] \\
& \text { with } V(0, T)=V_{o} \exp (\alpha T) ; \quad B(T)=B_{o} \exp \left(-B_{1} T\right)
\end{aligned}
$$

where $C=0.0894$ is treated as a universal constant, $\alpha$ is the thermal expansion coefficient, and $B(T)$ is known as the Tait parameter. This renders the Tait 
Table 2.4 A list of polymers (homopolymers and random copolymers), the S-S characteristic parameters $\left(P^{*}, V^{*}\right.$, and $\left.T^{*}\right)$, as well as the difference between the measured and computed volumes $(\Delta V)$ averaged over the data's temperature range $(\Delta T)$ and pressure range $(\Delta P)$ (Rodgers 1993a, b)

\begin{tabular}{|c|c|c|c|c|c|}
\hline Polymer & & $P^{*}(\mathrm{bar})$ & $T^{*}(\mathrm{~K})$ & $V^{*}(\mathrm{~mL} / \mathrm{g})$ & $\begin{array}{l}\Delta V(\Delta T, \Delta P)(\mu \mathrm{L} / \mathrm{g}) \\
\left({ }^{\circ} \mathrm{C}, \text { bar }\right)\end{array}$ \\
\hline \multicolumn{6}{|c|}{ Homopolymers } \\
\hline PDMS & Poly(dimethyl siloxane) & 5014 & 7864 & 0.9592 & $0.48(25-70,0-1000)$ \\
\hline PS & Polystyrene & 7159 & 12840 & 0.9634 & $0.35(115-196,0-2000)$ \\
\hline PoMS & Poly(o-methylstyrene) & 7461 & 13080 & 0.9814 & $0.46(139-198,0-1800)$ \\
\hline PMMA & Poly(methyl methacrylate) & 9264 & 11940 & 0.8369 & $0.10(114-159,0-2000)$ \\
\hline PcHMA & $\begin{array}{l}\text { Poly(cyclohexyl } \\
\text { methacrylate) }\end{array}$ & 7722 & 12030 & 0.9047 & $0.66(123-198,0-2000)$ \\
\hline PnBMA & Poly(n-butyl methacrylate) & 8560 & 10310 & 0.9358 & $1.31(34-200,0-2000)$ \\
\hline PMA & Poly(methyl acrylate) & 9691 & 10460 & 0.8431 & $0.93(37-220,0-1960)$ \\
\hline PVAc & Poly(vinyl acetate) & 9474 & 9348 & 0.8126 & $0.14(35-100,0-800)$ \\
\hline PTFE & Poly(tetrafluoroethylene) & 6581 & 8126 & 0.4339 & $1.36(330-372,0-390)$ \\
\hline PSF & Polysulfone & 11160 & 12770 & 0.7903 & $0.36(202-371,0-1960)$ \\
\hline PEG & Poly(ethylene oxide) & 9145 & 10150 & 0.8812 & $0.41(88-224,0-685)$ \\
\hline PTHF & Poly(tetrahydrofuran) & 7255 & 10280 & 1.0087 & $0.40(62-166,0-785)$ \\
\hline $\mathrm{BPE}$ & Branched polyethylene & 6923 & 10390 & 1.1674 & $0.99(125-198,0-2000)$ \\
\hline LPE & Linear polyethylene & 7864 & 9793 & 1.1406 & $0.90(142-200,0-2000)$ \\
\hline HMLPE & $\begin{array}{l}\text { High MW linear } \\
\text { polyethylene }\end{array}$ & 9213 & 9207 & 1.1278 & $1.10(137-200,0-2000)$ \\
\hline PIB & Polyisobutylene & 6866 & 11360 & 1.0940 & $0.20(53-110,0-1000)$ \\
\hline PMP & Poly(4-methyl-1-pentene) & 5453 & 11030 & 1.2050 & $1.79(241-319,0-1960)$ \\
\hline PBD & cis-1,4-polybutadiene & 8150 & 9225 & 1.0766 & $0.36(4-55,0-2835)$ \\
\hline HDPE & High-density polyethylene & 5063 & 12290 & 1.2190 & $1.43(140-203,0-1960)$ \\
\hline LDPE & Low-density polyethylene & 7936 & 9595 & 1.1380 & $2.26(121-175,0-1960)$ \\
\hline LDPE-A & $\begin{array}{l}\text { Low-density } \\
\text { polyethylene "A", }\end{array}$ & 7162 & 10580 & 1.1664 & $0.65(112-225,0-1960)$ \\
\hline LDPE-B & $\begin{array}{l}\text { Low-density } \\
\text { polyethylene "B" }\end{array}$ & 7036 & 10860 & 1.1734 & $0.59(112-225,0-1960)$ \\
\hline LDPE-C & $\begin{array}{l}\text { Low-density } \\
\text { polyethylene "C", }\end{array}$ & 7188 & 10660 & 1.1679 & $0.61(112-225,0-1960)$ \\
\hline $\mathrm{a}-\mathrm{PP}$ & a-Polypropylene & 6277 & 9494 & 1.1274 & $0.74(80-120,0-1000)$ \\
\hline i-PP & i-Polypropylene & 5730 & 11060 & 1.1884 & $1.26(170-297,0-1960)$ \\
\hline $\mathrm{i}-\mathrm{PB}$ & i-Poly(1-butene) & 6037 & 10920 & 1.1666 & $0.75(133-246,0-1960)$ \\
\hline PET & Poly(ethylene terephthalate) & 11940 & 11800 & 0.7426 & $0.57(274-342,0-1960)$ \\
\hline PPE & $\begin{array}{l}\text { Poly(2,6-dimethyl } \\
\text { phenylene oxide) }\end{array}$ & 9294 & 10580 & 0.8602 & $0.91(203-320,0-1765)$ \\
\hline $\mathrm{PC}$ & Bisphenol-A polycarbonate & 10200 & 11830 & 0.8156 & $0.37(151-340,0-1765)$ \\
\hline $\mathrm{PAr}$ & Polyarylate (Ardel) & 10030 & 12390 & 0.8091 & $0.26(177-310,0-1765)$ \\
\hline Phenoxy & Phenoxy & 11390 & 11730 & 0.8529 & $0.35(68-300,0-1765)$ \\
\hline PEEK & Poly(ether ether ketone) & 10860 & 12580 & 0.7705 & $0.85(346-398,0-2000)$ \\
\hline PVME & Poly(vinyl methyl ether) & 8481 & 10360 & 0.9632 & $0.68(30-198,0-2000)$ \\
\hline PA-6 & Polyamide 6 & 5499 & 16870 & 0.8327 & $0.44(236-296,0-1960)$ \\
\hline
\end{tabular}


Table 2.4 (continued)

\begin{tabular}{|c|c|c|c|c|c|}
\hline Polymer & & $P^{*}$ (bar) & $T^{*}(\mathrm{~K})$ & $V^{*}(\mathrm{~mL} / \mathrm{g})$ & $\begin{array}{l}\Delta V(\Delta T, \Delta P)(\mu \mathrm{L} / \mathrm{g}) \\
\left({ }^{\circ} \mathrm{C}, \text { bar }\right)\end{array}$ \\
\hline PA-66 & Polyamide 6,6 & 7069 & 12640 & 0.8195 & $0.52(246-298,0-1960)$ \\
\hline PEA & Poly(ethyl acrylate) & 8308 & 10040 & 0.8773 & $0.88(37-217,0-1960)$ \\
\hline PEMA & Poly(ethyl methacrylate) & 9870 & 10190 & 0.8710 & $0.81(113-161,0-1960)$ \\
\hline TMPC & Tetramethyl bisphenol-A PC & 8192 & 11540 & 0.8794 & $0.96(218-290,0-1600)$ \\
\hline HFPC & Hexafluoro bisphenol-A PC & 8510 & 10550 & 0.6317 & $0.66(159-280,0-2000)$ \\
\hline $\mathrm{BCPC}$ & Bisphenol chloral PC & 9878 & 12190 & 0.6975 & $0.47(155-284,0-2000)$ \\
\hline PECH & Poly(epichlorohydrin) & 9131 & 11370 & 0.7343 & $0.31(60-140,0-2000)$ \\
\hline PCL & Poly( $\varepsilon$-caprolactone $)$ & 7845 & 10870 & 0.9173 & $0.30(100-148,0-2000)$ \\
\hline $\mathrm{PVC}$ & Poly(vinyl chloride) & 8495 & 12350 & 0.7230 & $0.42(100-150,0-2000)$ \\
\hline \multicolumn{6}{|c|}{ Random copolymers } \\
\hline EP50 & Ethylene/propylene $50 \%$ & 5720 & 12220 & 1.2227 & $1.58(140-250,0-625)$ \\
\hline EVAc18 & Ethylene/vinyl acetate $18 \%$ & 7056 & 10630 & 1.1341 & $0.47(112-219,0-1765)$ \\
\hline EVAc25 & Ethylene/vinyl acetate $25 \%$ & 6978 & 10440 & 1.1040 & $0.56(94-233,0-1765)$ \\
\hline EVAc28 & Ethylene/vinyl acetate $28 \%$ & 7472 & 10310 & 1.0949 & $0.67(94-233,0-1765)$ \\
\hline EVAc40 & Ethylene/vinyl acetate $40 \%$ & 7539 & 10360 & 1.0446 & $0.54(75-235,0-1765)$ \\
\hline SAN3 & Styrene/acrylonitrile $2.7 \%$ & 7642 & 12070 & 0.9416 & $0.81(105-266,0-2000)$ \\
\hline SAN6 & Styrene/acrylonitrile $5.7 \%$ & 8238 & 11490 & 0.9352 & $0.70(96-267,0-2000)$ \\
\hline SAN15 & Styrene/acrylonitrile $15.3 \%$ & 7792 & 12360 & 0.9299 & $0.50(132-262,0-2000)$ \\
\hline SAN18 & Styrene/acrylonitrile $18 \%$ & 7853 & 12380 & 0.9255 & $0.42(104-255,0-2000)$ \\
\hline SAN40 & Styrene/acrylonitrile $40 \%$ & 8118 & 12900 & 0.9124 & $0.56(100-255,0-2000)$ \\
\hline SAN70 & Styrene/acrylonitrile $70 \%$ & 8747 & 13790 & 0.8906 & $0.36(100-270,0-2000)$ \\
\hline SMMA20 & $\begin{array}{l}\text { Styrene/methyl } \\
\text { methacrylate } 20 \%\end{array}$ & 7640 & 11800 & 0.9186 & $0.42(110-270,0-2000)$ \\
\hline SMMA60 & $\begin{array}{l}\text { Styrene/methyl } \\
\text { methacrylate } 60 \%\end{array}$ & 7911 & 11780 & 0.8739 & $0.55(110-270,0-2000)$ \\
\hline
\end{tabular}

Fig. 2.1 Frozen fraction of free volume $(F F)$ versus glass transition temperature $\left(T_{g}\right)$. Triangles: values for different polymers at ambient pressures (Simha and Wilson 1973). Squares: PS data at pressures P: 0-400 MPa (Data: Rehage 1980; calculations Utracki and Simha 1997)

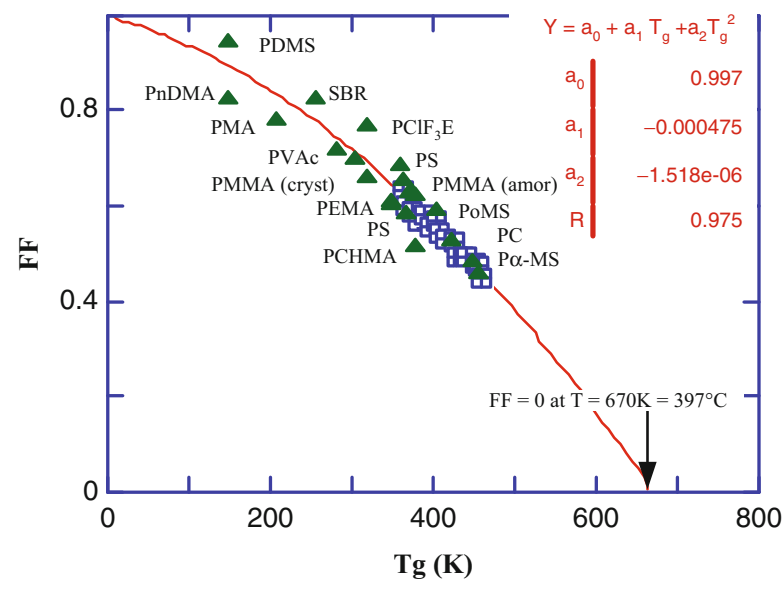


equation as a 4-parameter $\left(V_{o}, \alpha, B_{o}, B_{1}\right)$ representation of the $P V T$ behavior. About a century later, the H-H empirical PVT relation was proposed (Hartmann and Haque 1985), which is a three-parameter empirical EoS approach, that is, it has the form of a dimensionless EoS connecting $\tilde{P} \tilde{V} \tilde{T}$, with the usual definition of $\tilde{P} \equiv P / P^{*}, \tilde{V} \equiv V / V^{*}, \tilde{T} \equiv T / T^{*}$ and by using characteristic state values $\left(P^{*}, V^{*}\right.$, and $T^{*}$ ) for a given polymer. The $\mathrm{H}-\mathrm{H}$ relation is noteworthy due to the simplicity of its form and respectable agreement with the experimental data:

$$
\begin{aligned}
& \tilde{P} \tilde{V}^{5}=\tilde{T}^{3 / 2}-\ln \tilde{V} \\
& \text { with: } \quad V^{*}=V_{o} ; \quad P^{*}=B_{o} ; \quad T^{*}=T_{o}
\end{aligned}
$$

In Eq. 2.23, the characteristic pressure-reducing parameter, $B_{o}$, has been identified as the isothermal bulk modulus extrapolated to $T=0$ and $P=0$. Subsequently, Sanchez et al. (Sanchez 1993; Sanchez and Cho 1995) used a temperature-pressure (T-P) superposition which allows for the compressional strain, $\ln \left(V / V_{o}\right)$, to be written as a function of the reduced pressure, $\Delta P=\left(P-P_{o}\right) / B_{o}$, following the same general curve independent of T (here, $B$ is again the bulk modulus, and $B_{o}$ is its isothermal value evaluated at a reference pressure, $\left.P_{o}\right)$. The Sanchez-Cho $(\mathrm{S}-\mathrm{C})$ relation seems to provide the most faithful reproduction of experimental data among empirical three-parameter models. The S-C relation can be summarized as

$$
\begin{aligned}
& \tilde{V}=\frac{1}{1-\tilde{T}} \exp \left[\frac{\omega}{(1-\omega) B_{1}}\left\{1-\left(1+\frac{B_{1} \tilde{P}}{\omega} \exp (9 \tilde{T})\right)^{1-\omega}\right\}\right] \\
& \text { with }: \quad B_{1}=10.2 ; \quad \omega=0.9
\end{aligned}
$$

the corresponding $P^{*}, V^{*}$, and $T^{*}$ are tabulated for various polymers in Table 2.5. An excellent agreement with experimental data was achieved, indicating validity of the relation.

\subsubsection{Solid-Liquid and Vapor-Liquid Equilibria}

For a single component, the phase diagram provides a map of the solid, liquid, and vapor states, as well as their coexistence regions. It is customary to construct such single-component phase diagrams as $\log$-log plots of $P$ versus $V$ relation at constant $T$. Fig. 2.2 shows a phase diagram of argon. Dividing the three variables by the corresponding reducing parameters, the dependence can be cast in a general plot of reduced pressure versus reduced volume, $\tilde{P}$ versus $\tilde{V}$, at constant reduced temperature, $\tilde{T}$. For simple low molecular weight liquids, the van der Waals equation, Eq. 2.13, provides a reasonable description.

Guggenheim further demonstrated that near the critical point the coexistence curve of liquid and vapor follows a simple proportionality:

$$
\tilde{T}-1 \propto\left|(1-\widetilde{\rho})^{3}\right| / 3
$$

This dependence is shown in Fig. 2.3 as a solid line - the dashed line represents the van der Waals parabolic prediction. 
Table 2.5 Characteristic parameters $\left(P^{*}, V^{*}\right.$, and $\left.T^{*}\right)$ for the Sanchez-Cho empirical EoS model for various polymers (Cho 1999)

\begin{tabular}{|c|c|c|c|c|}
\hline Polymer & (Monomer or description) & $P^{*}($ bar $)$ & $T^{*}(\mathrm{~K})$ & $V^{*}(\mathrm{~mL} / \mathrm{g})$ \\
\hline PDMS & Dimethyl siloxane & 62129 & 1375.1 & 0.8071 \\
\hline PS & Styrene & 78676 & 2277.2 & 0.8165 \\
\hline PoMS & $o$-Methylstyrene & 77721 & 2380.6 & 0.8368 \\
\hline PMMA & Methyl methacrylate & 98730 & 2184.2 & 0.7139 \\
\hline PBMA & rc-Butyl methacrylate & 90253 & 1855.9 & 0.7963 \\
\hline PCHMA & Cyclohexyl methacrylate & 85151 & 2195.1 & 0.7710 \\
\hline PEA & Ethyl acrylate & 91318 & 1747.4 & 0.7398 \\
\hline PEMA & Ethyl methacrylate & 112570 & 1771.2 & 0.7332 \\
\hline PMA & Methyl acrylate & 106239 & 1829.0 & 0.7121 \\
\hline PVAC & Vinyl acetate & 103999 & 1696.9 & 0.6918 \\
\hline LPE & Linear polyethylene (PE) & 99435 & 1655.0 & 0.9491 \\
\hline $\mathrm{BPE}$ & Branched-PE & 86307 & 1751.9 & 0.9723 \\
\hline LDPE-A & Low-density PE-A & 82145 & 1865.4 & 0.9852 \\
\hline LDPE-B & Low-density PE-B & 79838 & 1923.8 & 0.9937 \\
\hline LDPE-C & Low-density PE-C & 82253 & 1880.5 & 0.9869 \\
\hline PBD & Butadiene & 94432 & 1633.8 & 0.9115 \\
\hline PBD8 & Butadiene with $8 \% 1,2$ content & 91363 & 1798.6 & 0.9308 \\
\hline PBD24 & Butadiene with $24 \% 1,2$ content & 87080 & 1819.0 & 0.9359 \\
\hline PBD40 & Butadiene with $40 \% 1,2$ content & 83520 & 1842.9 & 0.9357 \\
\hline PBD50 & Butadiene with $50 \% 1,2$ content & 77573 & 1892.0 & 0.9408 \\
\hline PBD87 & Butadiene with $87 \% 1,2$ content & 72418 & 1905.6 & 0.9498 \\
\hline $\mathrm{PB}$ & 1-Butene & 68911 & 1924.1 & 0.9854 \\
\hline PAr & Arylate & 115573 & 2243.9 & 0.6839 \\
\hline PCL & Caprolactone & 95301 & 1849.0 & 0.7671 \\
\hline $\mathrm{PC}$ & Carbonate (PC) & 121061 & 2070.3 & 0.6871 \\
\hline $\mathrm{BCPC}$ & Bisphenol chloral PC & 99313 & 2249.1 & 0.5971 \\
\hline HFPC & Hexafluoro bisphenol-A PC & 101731 & 1788.2 & 0.5264 \\
\hline TMPC & Tetramethyl bisphenol-A PC & 105730 & 1908.0 & 0.7261 \\
\hline PET & Ethylene terephthalate & 152788 & 2022.2 & 0.6199 \\
\hline PIB & Isobutylene & 70453 & 2130.2 & 0.9382 \\
\hline PI8 & Isoprene with $8 \% 3,4$ content & 76696 & 1921.0 & 0.9453 \\
\hline PI14 & Isoprene with $14 \% 3,4$ content & 82199 & 1911.3 & 0.9366 \\
\hline PI41 & Isoprene with $41 \% 3,4$ content & 81123 & 1912.7 & 0.9370 \\
\hline PI56 & Isoprene with $56 \% 3,4$ content & 86001 & 1854.5 & 0.9330 \\
\hline i-PP & Isotactic polypropylene & 61181 & 1991.5 & 1.0116 \\
\hline $\mathrm{a}-\mathrm{PP}$ & Atactic polypropylene & 62028 & 1776.2 & 0.9690 \\
\hline Phenoxy & Phenoxy & 127281 & 2103.4 & 0.7242 \\
\hline PSO & Sulfone & 132864 & 2232.2 & 0.6655 \\
\hline PEO & Ethylene oxide & 108054 & 1789.1 & 0.7441 \\
\hline PVME & Vinyl methyl ether & 92705 & 1861.3 & 0.8187 \\
\hline
\end{tabular}


Table 2.5 (continued)

\begin{tabular}{|c|c|c|c|c|}
\hline Polymer & (Monomer or description) & $P^{*}$ (bar) & $T^{*}(\mathrm{~K})$ & $V^{*}(\mathrm{~mL} / \mathrm{g})$ \\
\hline PEEK & Ether ether ketone & 143355 & 2126.5 & 0.6395 \\
\hline PTFE & Tetrafluoroethylene & 97575 & 1400.7 & 0.3638 \\
\hline PTHF & Tetrahydrofuran & 81602 & 1843.0 & 0.8561 \\
\hline PMP & 4-Methyl- $i$-pentene & 64525 & 1885.0 & 1.0089 \\
\hline PA6 & Amide 6 & 57184 & 3140.3 & 0.7130 \\
\hline PA66 & Amide 66 & 83919 & 2195.2 & 0.6887 \\
\hline PECH6 & Epichlorohydrine & 88993 & 2068.9 & 0.6269 \\
\hline PVC & Vinyl chloride & 75512 & 2395.4 & 0.6252 \\
\hline PPO & Phenylene oxide & 117769 & 1810.4 & 0.7181 \\
\hline EP50 & Ethylene/propylene $50 \%$ & 64210 & 2384.7 & 1.0582 \\
\hline EVAc18 & Ethylene/vinyl acetate $18 \%$ & 81075 & 1878.9 & 0.9585 \\
\hline EVAc25 & Ethylene/vinyl acetate $25 \%$ & 79575 & 1848.3 & 0.9338 \\
\hline EVAc28 & Ethylene/vinyl acetate $28 \%$ & 86221 & 1812.5 & 0.9241 \\
\hline EVAc40 & Ethylene/vinyl acetate $40 \%$ & 84419 & 1856.1 & 0.8864 \\
\hline SAN3 & Styrene/acrylonitrile $2.7 \%$ & 78785 & 2185.7 & 0.8030 \\
\hline SAN6 & Styrene/acrylonitrile $5.7 \%$ & 88968 & 2010.7 & 0.7896 \\
\hline SAN15 & Styrene/acrylonitrile $15.3 \%$ & 86020 & 2170.4 & 0.7860 \\
\hline SAN18 & Styrene/acrylonitrile $18 \%$ & 84956 & 2208.4 & 0.7854 \\
\hline SAN40 & Styrene/acrylonitrile $40 \%$ & 77726 & 2435.2 & 0.7853 \\
\hline SAN70 & Styrene/acrylonitrile $70 \%$ & 91770 & 2546.6 & 0.7616 \\
\hline SMMA20 & Styrene/methyl methacrylate $20 \%$ & 85313 & 2105.6 & 0.7789 \\
\hline SMMA60 & Styrene/methyl methacrylate $60 \%$ & 88589 & 2099.5 & 0.7408 \\
\hline
\end{tabular}

\subsubsection{Gibbs Phase Rule}

The state variables are those intensive or extensive quantities that describe a system, for example, by means of the "equation of state." The total number of variables required to describe a system with $i$ number of components is $i+2$ (cf. Eq. 2.11, $i$ accounts for the $i$ composition variables, $N_{i}$, and 2 accounts for, e.g., $P$ and $T$ ). For the discussions of phase diagrams, it is important to know how many of the state variables can be varied without going through a phase transition. For a closed system with $i$ number of components and $\Pi$ number of phases, the number of intensive variables (cf. thermodynamic degrees of freedom, $\# f$ ) is given by the "Gibbs phase rule":

$$
\# f=i+2-\Pi
$$

For example, for a phase equilibrium of a two-component blend $(i=2)$, in order to follow the two-phase $(\Pi=2)$ coexistence, Eq. 2.26 predicts $\# f=2$, i.e., two 

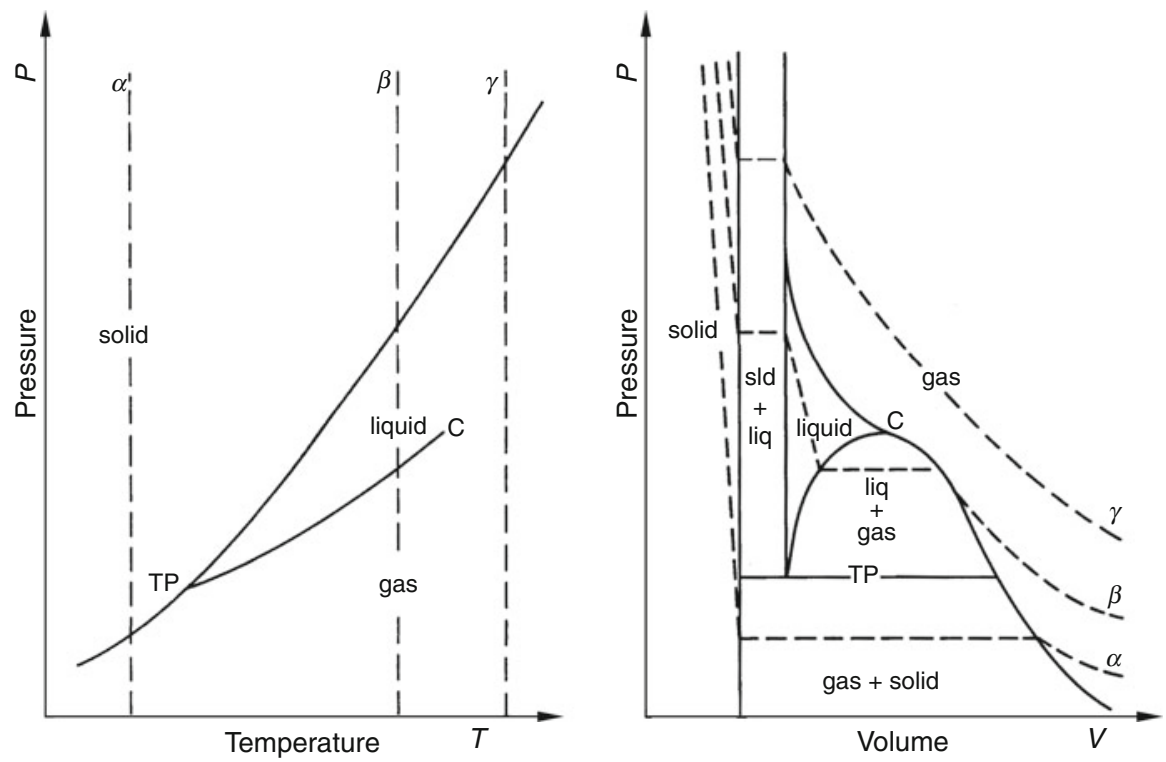

Fig. 2.2 The van der Waals-type phase diagram in T-P (left) and P-V (right) axes. $C$ is the critical point; $T P$ is the triple point of the coexistence between solid, liquid, and vapor (TP is a line in the P-V phase diagram). Solid lines are the phase boundaries, whereas the dashed lines are isotherms at different temperatures $\left(\alpha\right.$ below $T_{C}, \beta$ slightly below $\mathrm{T}_{C}$, and $\gamma$ above $T_{C}$ ). The liquid-gas, solid-liquid, and solid-vapor coexistence regions are also shown (After Flowers and Mendoza 1970)

Fig. 2.3 The liquid-vapor coexistence curves, $\tilde{T}$ versus $\widetilde{\rho}$, as predicted by Guggenheim (solid line) and by van der Waals

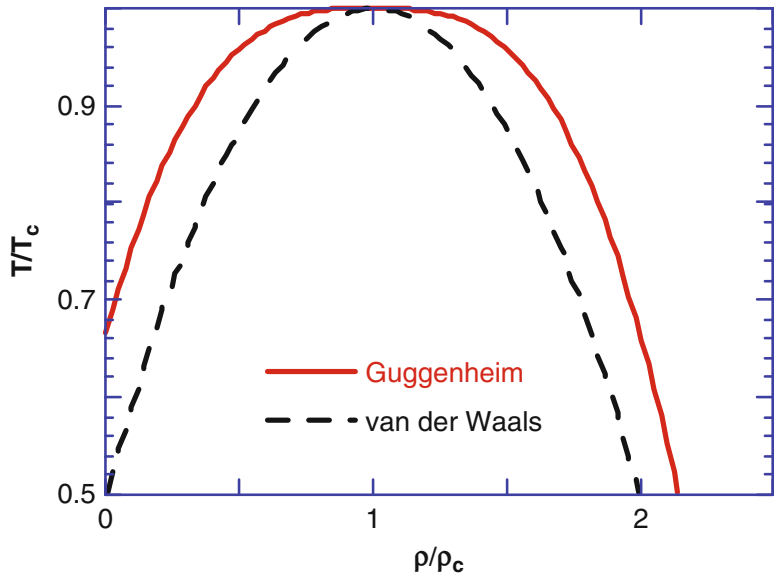

variables must be simultaneously changed (e.g., $T$ and composition), whereas, for the same system within the single-phase $(\Pi=1)$ miscible region, three variables are available (e.g., composition, $T$ and $P$ ). For a discussion based on general phase equilibria conditions, see Sect. 1.2.3 (Klenin 1999). 


\subsection{Polymeric Mixtures}

Polymeric mixtures are conveniently divided into polymer solutions (containing one or more low molecular weight liquids, termed as solvent) and polymer blends (containing only macromolecular species). They will be briefly described in the following Sects. 2.5.1 and 2.5.2, respectively. More detailed discussion of the theoretical foundations is given in Sect. 2.6.

\subsubsection{Polymer Solutions}

In contrast to the mixtures of low molecular weight species, where composition is customarily expressed as a concentration or mole fraction, $x_{i}$, in the case of polymer solutions, composition must be given as volume fraction, $\phi_{i}$, to correctly account for the much larger space (volume) occupied by a given concentration of a polymer, cf. for a polymer with degree of polymerization $n, \phi_{i} \sim x_{i} n$. For dilute solutions, the $\mathrm{wt} / \mathrm{vol}$ concentration, $c$, can be also used accounting for weight of polymer per volume of solvent (e.g., in g/100 mL). As in mixtures of small molecules, also in polymer solutions, the solubility originates mainly from entropic reasons (Yamakawa 1971).

Traditionally, solutions have been used in polymer characterization, e.g., to measure its molecular weight averages (number, weight, and z-averaged molecular weight, $M_{n}, M_{w}$, and $M_{z}$ ), or the size of its macromolecular coil. The latter may be expressed as the unperturbed end-to-end distance $\left(R_{o}\right)$, or through the related radius of gyration $\left(R_{g}, o\right)$, viz.,

$$
\left\langle R_{o}^{2}\right\rangle=6\left\langle R_{g, o}^{2}\right\rangle=\sigma^{2} n l^{2} \frac{1+\langle\cos \theta\rangle}{1-\langle\cos \theta\rangle}
$$

In Eq. 2.31, $\sigma$ is the steric hindrance factor of the macromolecular chain, $n$ is the number of statistical segments (each statistical segment having a length $l$ and the bond angle between two consecutive segments is equal to $\theta$ ).

Solutions can also be used to measure of the thermodynamic interactions between polymer segments and solvent molecules. These intermolecular interactions are best discussed in terms of the virial coefficients, $A_{i}$. The change of the solvent chemical potential upon dissolution of a polymer is given by

$$
\Delta \mu=\left(\frac{\partial G_{m}}{\partial N_{2}}\right)_{T, P, N_{1}}=R T \ln a_{o}=-V_{1} \Pi
$$

where $a_{o}$ is the activity and $\Pi$ the osmotic pressure, usually expressed as

$$
\frac{\Pi}{c}=R T\left(\frac{1}{M_{n}}+A_{2} c+A_{3} c^{2}+\ldots\right) \quad \text { with } \quad A_{2}=\frac{4 \pi^{3 / 2} N_{A}}{M_{n}^{2}}\left\langle R_{g}^{2}\right\rangle^{3 / 2} \Psi(z)
$$

The second virial coefficient in Eq. 2.29 contains two functionals that depend on the binary interactions, the perturbed radius of gyration $R_{g}$, and the function $\Psi(z)$, which is 


$$
\Psi=\frac{1-\exp (-5.73 z)}{5.73} \quad \text { with } \quad z=\frac{4}{3^{3 / 2}} C_{M}\left(\frac{1}{2}-\chi\right) M_{n}^{1 / 2}
$$

As $z$ increases from 0 (theta conditions) to 2 (good solvent), the $\Psi$ function also increases from 0 to about 0.2 . For simplicity, dilute solutions are used to avoid the need for determination of higher-order virial coefficients. In this case, $A_{2}$ provides a direct measure of the intermolecular interactions in polymer solutions and can be directly related to the respective Flory-Huggins parameter ( $\chi$, vide infra, Sect. 2.6.1.1). However, since $A_{3} \propto A_{2} M_{n}$, Eq. 2.29 can be written as

$$
\left(\frac{\Pi M_{n}}{R T}\right)^{1 / 2}=1+\frac{M_{n} A_{2}}{2}
$$

In another approach, light scattering also makes it possible to determine $A_{2}$, viz.,

$$
\frac{K c}{R_{\theta}}=\frac{1}{M_{w}\left\langle P_{1}(\theta)\right\rangle}+2 A_{2}^{\prime} c+3 A_{3}^{\prime} c^{2}+\ldots
$$

where $K$ is an experimental quantity $\left(K=4 \pi^{2}(d n / d c)^{2} \eta_{o}^{2}\left(1+\cos ^{2} \theta\right) / N_{A} \lambda^{4}\right)$, $c$ is the concentration of the solution, $R_{\Theta}(c)$ is the Rayleigh ratio, and $\left\langle P_{1}(\theta)\right\rangle$ is the intramolecular interference factor, i.e., the angular dependence of the scattered light. Equation 2.32 provides the polymer's molecular weight, $M_{w}$, from the dilute solution limit where all higher-order terms of the virial expansion become negligible: $\lim _{c \rightarrow 0, \theta \rightarrow 0}\left(K c / R_{\theta}\right)=1 / M_{w}$. Similar to the osmotic pressure (Eq. 2.29), light scattering (Eq. 2.32) also allows to calculate virial coefficients, with the second virial coefficient again being related to the solvent-solute interactions.

The thermodynamic interactions and the size of polymer coil also enter dependencies that describe the transport behavior of polymer solutions, viz., viscosity, diffusion, sedimentation, etc. To complete this short summary, the viscosity relations should be mentioned. Defining $\eta$ as the solution viscosity and $\eta_{o}$ as the solvent viscosity, the following, relative $\left(\eta_{r}\right)$, specific $\left(\eta_{s p}\right)$, and intrinsic $([\eta])$ viscosities, are typically expressed as

$$
\begin{aligned}
& \eta_{r} \equiv \eta / \eta_{o} \\
& \eta_{s p} \equiv\left(\eta-\eta_{o}\right) / \eta_{o}=\eta_{r}-1 \\
& {[\eta] \equiv \lim _{c \rightarrow 0}\left(\eta_{s p} / c\right)=\lim _{c \rightarrow 0} \frac{\ln \eta_{r}}{c}} \\
& \eta_{s p} / c \cong[\eta]+k_{H}[\eta]^{2} c \quad \text { (Huggins equation) }
\end{aligned}
$$

which can also be fitted to a virial-type expansion (e.g., $\left.\eta_{r}=1+[\eta] c+k^{\prime}([\eta] c)^{2}+\cdots\right)$. Many relations have been proposed connecting the intrinsic viscosity, $[\eta]$, to the polymer/solvent interaction parameters. One of the better known is that credited to Inagaki et al. (1966): 


$$
[\eta]^{4 / 5} / M_{v}^{2 / 5}=0.786 K^{4 / 5}+0.454 K^{2 / 15} \Phi_{o}^{2 / 3} B^{2 / 3} M_{v}^{1 / 3}
$$

where $K=2.5 \times 10^{23}\left[\left\langle R_{o}^{2}\right\rangle / M_{v}\right]^{3 / 2}$ and $B=\left(2 \rho_{s}^{2} / V_{o}\right)(0.5-\chi)$, with $\rho_{s}$ and $V_{o}$ being the segmental density and the molar volume of the solvent.

It is worth noting that the three methods of evaluation of the solution behavior, osmometry, light scattering, and intrinsic viscosity, provide different molecular weight averages, respectively, number average, $M_{n}$, weight average $M_{w}$, and "viscosity" average, $M_{v}$. Knowing at least two of them, one can also estimate the width of the molecular weight distribution through polydispersity factors, e.g., through $M_{w} / M_{n}$.

\subsubsection{Polymer Blends: Definitions and Miscibility}

In contrast to solutions, polymer blends are mostly immiscible. As shown in Appendix 2 by Krause and Goh, over 1,000 cases of miscibility have been found; however, these are so infrequent and poorly defined that one may consider them as exceptions to the general rule of polymer/polymer immiscibility.

While the thermodynamic definition of miscibility is unambiguous (see Table 2.6), there is a significant amount of discussion as to the methods of miscibility detection and the size of heterogeneity in miscible blends.

The methods of miscibility detection (sometimes wrongly labeled as "compatibility" experiments) will be discussed later in this chapter. Opacity (turbidity) can provide limited only information on miscibility - since light scattering manifests when the size of heterogeneity becomes larger than $100 \mathrm{~nm}$ and the difference in refractive index is greater than about 0.01; further complications arise when one or both polymer phases are semicrystalline. The most widely used tool for the "detection of miscibility" relates to measurement of the glass transition temperature, $T_{g}$. There is a widely accepted belief that blends which display a single $T_{g}$ are miscible. The glass transition temperature is relatively simple to measure, but there are inherent uncertainties of the measurements that need to be carefully examined (Utracki 1989). For example, $T_{g}$ is insensitive when the amount of one component is less than about $10 \mathrm{wt} \%$ or when the component $T_{g}$ 's occur at similar temperatures. On the latter, the $T_{g}$ method should not be used for blends containing polymers whose $T_{g}$ 's differ by less than $10{ }^{\circ} \mathrm{C}$ from each other.

Along these lines, it has been shown, first by Schultz and Young (1980) and then by many others, that $T_{g}$ is not sensitive to the thermodynamic miscibility of the components, but rather to the degree of dispersion. For example, in solvent-mixed PS/PMMA blends that were not allowed to phase separate, a single $T_{g}$ has been detected, but when the specimens were annealed, double peaks were observed. In another example, solvent cast blends of PVC with caprolactone-grafted lignin showed a single $T_{g}$, while the measured domain size ranged between 10 and $30 \mathrm{~nm}$, indicating immiscibility (De Oliveira and Glasser 1994). 
Table 2.6 Terms related to polymer blend miscibility (see also $>$ Chap. 1, "Polymer Blends: Introduction")

Miscible polymer blend: polymer blend, homogenous down to the molecular level, in which the domain size is comparable to the macromolecular dimension; associated with negative value of the free energy of mixing, $\Delta G_{m} \simeq \Delta H_{m} \leq 0$, and within the phase stability condition $\partial^{2} \Delta G_{m} / \partial^{2} \phi>0$

Immiscible blends: polymer blends whose free energy increases upon mixing, i.e., $\Delta G_{m} \simeq$ $\Delta H_{m}>0$

Polymer alloy: immiscible but compatibilized polymer blend; implies a modified interphase and, thus, morphology

Interphase: a nominal third phase in binary polymer alloys, engendered by interdiffusion or compatibilization at the interfaces between the two polymer domains. The interphase thickness $\Delta l$ varies between 1 and $60 \mathrm{~nm}$ depending on polymers' miscibility and compatibilization

Compatibilization: process of modification of the interphase in immiscible polymer blends, resulting in reduction of the interfacial energy, development, and stabilization of a desired morphology, leading to the creation of a polymer alloy with enhanced performance

Fig. 2.4 The glass transition temperature versus composition for PEK/N-TPI blends. The two black squares, connected by a straight line, are the $T_{g}$ 's of the two polymers; open circles are data for blends prepared at $455{ }^{\circ} \mathrm{C}$; solid circles are for the same blends prepared at $400{ }^{\circ} \mathrm{C}$. The lines are only guides to the eye (Data from Sauer et al. 1996)

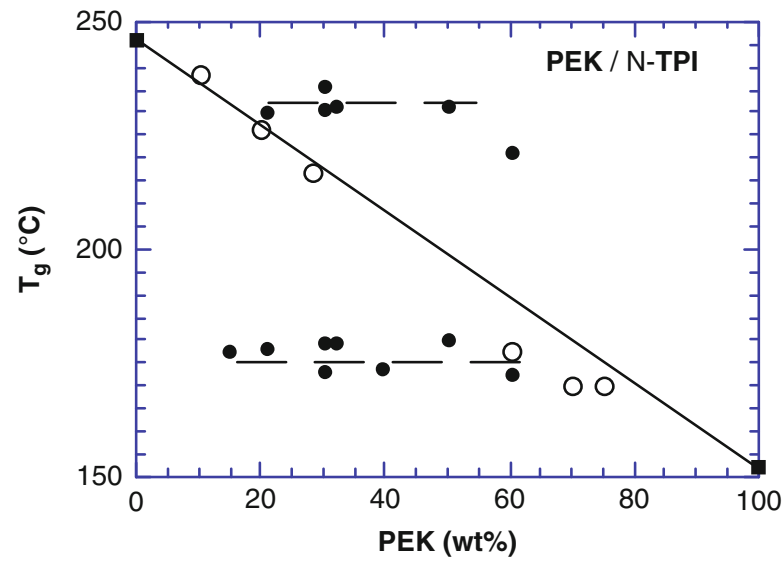

For PEK/PI blends, the $T_{g}$ 's of the neat components are separated by about $90{ }^{\circ} \mathrm{C}$; however, depending on the chemical nature of the components, as well as on the blend composition and blending conditions, three types of the glass transition behavior were observed: Specifically, three $T_{g}$ behaviors for PEK blended with N-TPI were obtained, as shown in Fig. 2.4 after (Sauer et al. 1996) (N-TPI stands for: "new thermoplastic polyimide," obtained from condensation of 4,4-bis (3-aminophenoxy)biphenyl with pyromellitic dianhydride). At "low" blending temperature, of $400{ }^{\circ} \mathrm{C}$, the mixture showed two $T_{g}$ 's, but when the same polymers were blended at $T=440{ }^{\circ} \mathrm{C}$, the blend showed a single $T_{g}$; interestingly, when the two polymers were blended at intermediate temperatures, $400{ }^{\circ} \mathrm{C}<T<440{ }^{\circ} \mathrm{C}$, a single, broad, overlapping $T_{g}$ was obtained. Clearly, the glass transition behavior reflected different degrees of dispersion for the PEK/PI system, rather than phase miscibility (the degradation temperature is $410{ }^{\circ} \mathrm{C}$ ). Finally, it is frequent that blends of rubber-containing copolymers (such as SBS or ABS type), e.g., PPE/SBS, 
PC/ABS, or PPE/MBS, are identified as miscible, on the basis of $T_{g}$ measurements. Obviously, this $T_{g}$ measurement probes the mixing of the viscoelastic components of the blend, effectively neglecting the elastomeric (rubber) domains. The elastomeric domains remain immiscible in the blends, much like they were in the copolymers, and may show (or not, depending on their content) another $T_{g}$ at much lower temperatures.

In any liquid, either single or multicomponent one, there is a significant amount of heterogeneity, usually expressed by density/composition fluctuation functions. Thus, along the above discussion, it is justified to ask two questions:

1. What is the maximum size, $d_{d}$, of heterogeneity in a polymer blend that fulfills the conditions of the thermodynamic miscibility (viz., $\Delta G_{m} \leq 0$ and $\left.\partial^{2} \Delta G_{m} / \partial^{2} \phi \geq 0\right)$ ?

2. At what level of heterogeneity $T_{g}$ is a monotonic function of composition?

Various answers have been given to the first question. On the basis of the size of the cooperative segmental motion required at $T_{g}$, it was estimated that $d_{d}$ is $2-3 \mathrm{~nm}$ (Boyer 1966; Warfield and Hartmann 1980). The local segmental dynamics of a flexible polymer chain was found to be affected by the neighboring chains lying within 2-7 nm (Callaghan and Paul 1994a, b). Other authors consider that miscibility is achieved when the heterogeneity diameter becomes comparable to the unperturbed radius of gyration of the macromolecule, thus $d_{d}=\left\langle R_{g}^{2}, o\right\rangle^{1 / 2}$ typically 3-10 nm (Silberberg and Kuhn 1952; Wolf 1980, 1984). Many others' estimates fall within these limits (e.g., Kaplan 1976; Bair and Warren 1980; Cowie 1989). Thus, it seems that the thermodynamic miscibility is associated with a size of compositional heterogeneity $d_{d}$ smaller than about $10 \mathrm{~nm}$, cf. smaller than the polymer size, an intuitively expected answer for intimate mixing of chaintype objects.

The answer to the second question has been given as well. Depending on the chemical nature of the system and its morphology, double peak of $T_{g}$ has been reported to appear for domain sizes $d_{d}$ as small as 15-20 nm (Frisch et al. 1982; Utracki 1989; De Oliveira and Glasser 1994).

From a fundamental point of view, the glass transition reflects a change in the molecular mobility upon cooling and can be viewed as associated with "freezing" of a portion of the free volume. However, the frozen fraction depends on the absolute value of $T_{g}$-as the $T_{g}$ absolute value increases, more free volume becomes accessible to the thermal motion in the glassy state. Judging by data presented in Fig. 2.1, for organic macromolecules, the fundamental mechanism of the glass transition is not expected to be valid for $T_{g}$ larger than about $400{ }^{\circ} \mathrm{C}$. In short, even under the most favorable conditions, $T_{g}$ should be unable to discriminate between the presence and absence of thermodynamic miscibility, i.e., when a system is miscible, a single $T_{g}$ will indeed be found, but also a single $T_{g}$ will also be manifested for immiscible systems having finely dispersed phases. In many cases, $T_{g}$ may be able to detect the "technological miscibility," i.e., to identify systems that are so well homogenized that the phase domains will not be affected by the processing conditions. The method may be used as a pragmatic scan for industrially useful blends, or evaluation of a compatibilization scheme. 
It is noteworthy that "homogeneity at fairly fine level is necessary for optimum performance, but some degree of microheterogeneity is usually desirable to preserve the individual properties of respective polymer components" (Hess et al. 1993). Note that nearly all commercial polymer blends (with the notable exception of the PVDF/PMMA blend) are immiscible. One tends to study miscibility not so much as to develop single-phase commercial blends, but mainly to design better compatibilizers and compatibilization strategies.

Since the standard test methods for detecting miscibility, viz., $T_{g}$ measurements, microscopy, small-angle X-ray scattering, etc., are limited to $d_{d} \geq 15 \mathrm{~nm}$, other methods should be used for studies of true thermodynamic miscibility. Such approaches mostly capitalize on advanced scattering methods, such as high-resolution or multidimensional nuclear magnetic resonance (NMR) techniques, spin-diffusion NMR measurements, non-radiative energy transfer, excimer fluorescence, thermally stimulated depolarization current, small-angle neutron scattering (SANS), Fourier transfer infrared spectroscopy (FTIR), etc. Even simple NMR measurement of spin-lattice relaxation times, $T_{1}$, is capable to distinguish down to domain sizes of 2-3 nm. The method can be used for either molten or solidified mixture specimens. For example, these methods produced the following results:

a-PVC/PMMA: Homogenous at $20 \mathrm{~nm}$, but heterogeneous at $2 \mathrm{~nm}$. After deuteration of PMMA, the miscibility extended down to $1-2 \mathrm{~nm}$.

PS/PVME, PnBMA/PS (2 mol\% -OH): Homogenous at $20 \mathrm{~nm}$.

SAN/PMMA: Homogenous at $20 \mathrm{~nm}$, but heterogeneous at 2-15 nm (McBrierty et al. 1978).

a-PET/PC, a-PVDF/PMMA, a-PCL/PVC: Homogenous at $2 \mathrm{~nm}$ (Douglass and McBrierty 1978; Douglass 1979).

PS/PPE: Only about $30 \mathrm{wt} \%$ blend components participate in intimate interactions on the scale of less than $2 \mathrm{~nm}$. The rest show the same nuclear resonance pattern as the one recorded for the two homopolymers (Stejskal et al. 1981; Takahashi et al. 1990).

\subsection{Theories of Liquid Mixtures}

\subsubsection{Lattice, Cell, and Hole Theories}

The statistical mechanics methods that use a pseudocrystalline model of regularly placed elements on a "lattice" are known as lattice theories. Many theories, known under the names of free volume, cell-hole, tunnel, Monte Carlo, or molecular dynamics belong here. Of these, only two will be mentioned. The first, and the best known, was originally developed by Huggins (1941) and, independently, by Flory (1941), then extended by many authors (Utracki 1962; Koningsveld 1967). The second is the cell-hole Simha and Somcynsky (1969) theory that has been incessantly evolving during the intervening years. The theory makes it possible to interpret and predict different material's behavior in a wide range of 
states and independent variables, viz., gases, low molecular weight organic liquids, metals, and polymers in a glassy or molten state, to determine miscibility of gases or liquids in polymers, to compute the phase diagrams of polymer blends, etc.

\subsubsection{Flory-Huggins Theory}

For binary systems that contain two components denoted as $i$ (i.e., $i=1$ or 2 and, traditionally, for polymer solutions the subscript 1 indicates solvent, and 2 polymer) the Flory-Huggins, FH, relation has been expressed in several equivalent forms:

$$
\begin{aligned}
\frac{\Delta G_{m}}{R T} & =\frac{\phi_{1}}{V_{1}} \ln \phi_{1}+\frac{\phi_{2}}{V_{2}} \ln \phi_{2}+\chi_{12}^{\prime} \phi_{1} \phi_{2} \quad\left[\text { with } \quad \chi_{12}^{\prime} \equiv \chi_{12} / V_{r e f}\right] \\
\Delta G_{m} & =R T V \quad\left[\frac{\phi_{1}}{V_{1}} \ln \phi_{1}+\frac{\phi_{2}}{V_{2}} \ln \phi_{2}\right]+B \phi_{1} \phi_{2} \quad\left[\text { with } \quad B \equiv \chi_{12} R T\left(V / V_{r e f}\right)\right]
\end{aligned}
$$

In Eq. 2.35, $\phi_{i}$ is the volume fraction and $V_{i}$ is the molar volume of component " $i$." The first two logarithmic terms give the combinatorial entropy of mixing, which are by definition of $\phi$ negative and always promote mixing, while the third term is the enthalpy of mixing. For polymer blends $V_{i}$ are both large; thus the combinatorial entropy becomes vanishingly small, and, therefore, the miscibility or immiscibility of the system is determined by the value of the last term, $\chi_{12}^{\prime} \phi_{1} \phi_{2}$. Using $\phi_{2}+\phi_{1}=1$ and the monomeric volume as a reference volume, the free energy of mixing $\Delta G_{m}$, expressed now in a per monomer basis, can be rewritten as

$$
\frac{\Delta G_{m}}{k T}=\frac{\phi}{N_{1}} \ln \phi+\frac{1-\phi}{N_{2}} \ln (1-\phi)+\chi_{12}^{\prime} \phi(1-\phi)
$$

where $N_{i}$ is the degree of polymerization of the $i$ component $\left(N_{i}=1\right.$ for $i$ being a solvent). For the purposes of determining phase behavior or miscibility, it does not matter if one uses the change in free energy of mixing expressed per unit volume, per mole of lattice sites, or per monomeric volume. Due to the assumption of the FH model, in its unaltered original form, the model predicts UCST only behavior (Fig. 2.5).

Applying to Eq. 2.35 the critical point conditions (the critical point is located on the spinodal, thus, $\partial^{2} \Delta G_{m} / \partial^{2} \phi=0$, and is the extremum of the spinodal curve, thus, $\left.\partial^{3} \Delta G_{m} / \partial^{3} \phi=0\right)$ and treating the so-called binary interaction parameter, $\chi_{12}$ or $B$, as composition independent, the critical conditions for phase separation can be expressed as

$$
\begin{aligned}
& \chi_{12, c r}^{\prime}=\frac{1}{2}\left(\frac{1}{\sqrt{V_{1}}}+\frac{1}{\sqrt{V_{2}}}\right)^{2}=\frac{1}{2}\left(\frac{1}{\sqrt{N_{1}}}+\frac{1}{\sqrt{N_{2}}}\right)^{2} \\
& B_{c r}=\frac{R T V}{2}\left(\frac{1}{\sqrt{V_{1}}}+\frac{1}{\sqrt{V_{2}}}\right)^{2}=\frac{R T}{2}\left(\frac{1}{\sqrt{N_{1}}}+\frac{1}{\sqrt{N_{2}}}\right)^{2}
\end{aligned}
$$




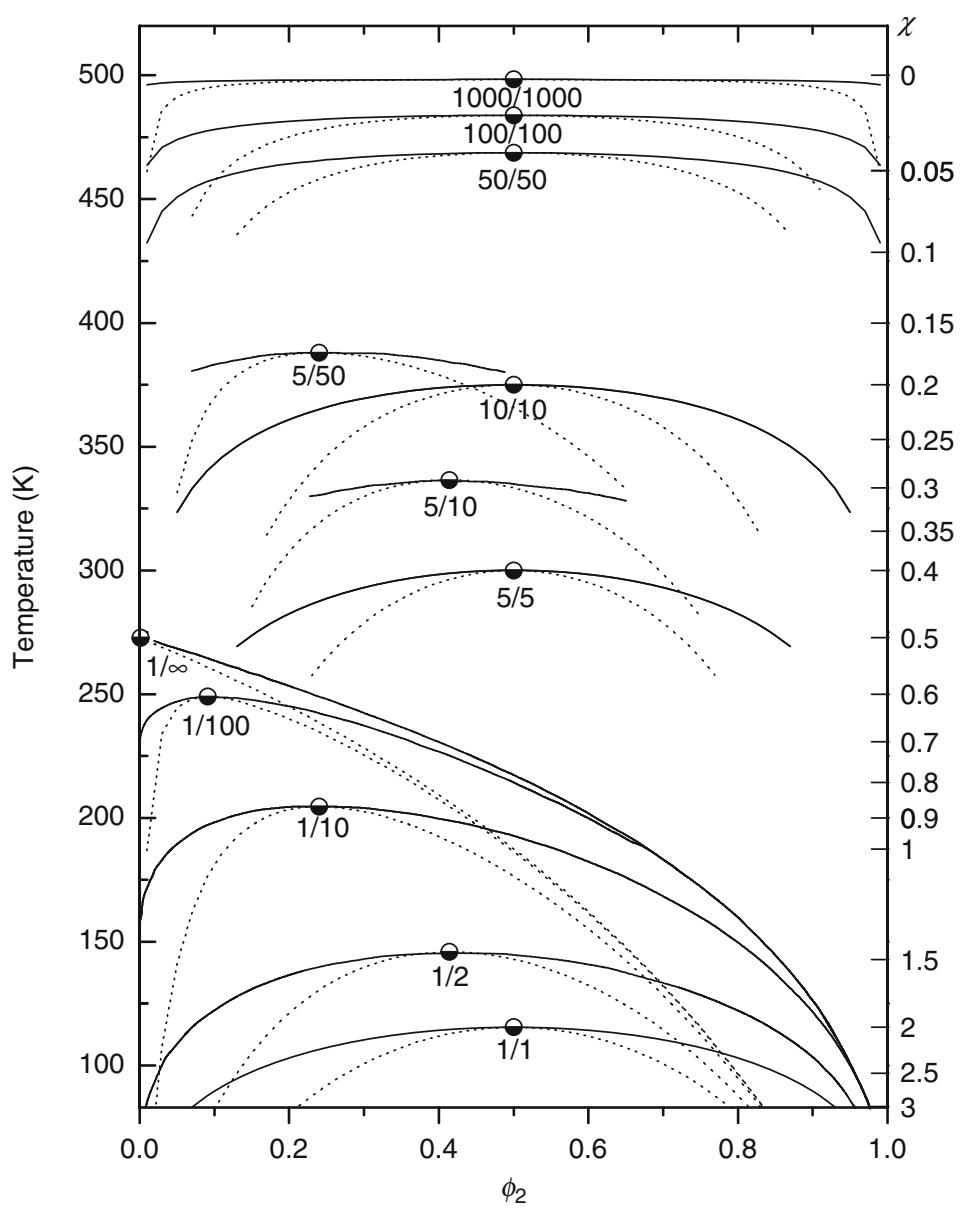

Fig. 2.5 Phase diagrams predicted by the Flory-Huggins $(F H)$ model for various ratios of molar sizes $\left(N_{1} / N_{2}\right.$, as indicated): solid lines are binodals and dashed lines are spinodals; for all phase diagrams, a generic $\chi_{12}=-0.6+300 / T$ was assumed; see right-side y-axis. Three groups of phase diagrams are distinguished: the group in the bottom corresponds to solutions, with component 1 being the solvent $\left(N_{1}=1\right)$; the middle group corresponds to mixtures of oligomeric molecules; and only for the top three phase diagrams correspond to mixtures that resemble polymer blends $\left(N_{1} N_{2} \gg 1\right)$. All spinodals, UCST critical points, and the binodals of the symmetric, $N_{1}=N_{2}$, mixtures were analytically calculated from the derivatives of Eq. 2.35 (critical points from Eq. 2.36), and the rest of the binodals are from numerical solutions

Equation 2.36 gives the miscibility conditions for systems with species of different molecular weight. The relations are rather accurate, as they are markedly insensitive to the FH assumptions and approximations (Fig. 2.6). Three special cases can be distinguished:

1. Small molecule mixtures $\left(V_{1} \simeq V_{2}\right.$ or $\left.N_{1}=N_{2} \approx 1\right)$ are miscible when $\chi_{12}<2$, viz., $\chi_{12}<\chi_{12, c r}$ and from Eq. $2.36 \chi_{12, c r}=2$ (for $N_{1}=N_{2}=1$ ). 
Fig. 2.6 Critical temperatures, both UCST and LCST, as a function of polymer $M_{w}$, for polystyrene solutions in methylcyclohexane. In the inset, the critical temperatures are fitted to Eqs. 2.36 and 2.39 (vide infra) showing an excellent agreement over the whole ensemble of data (Data from Wohlfarth (2008); collected from 71 systems, from 18 independent studies published from 1963 to 2002)

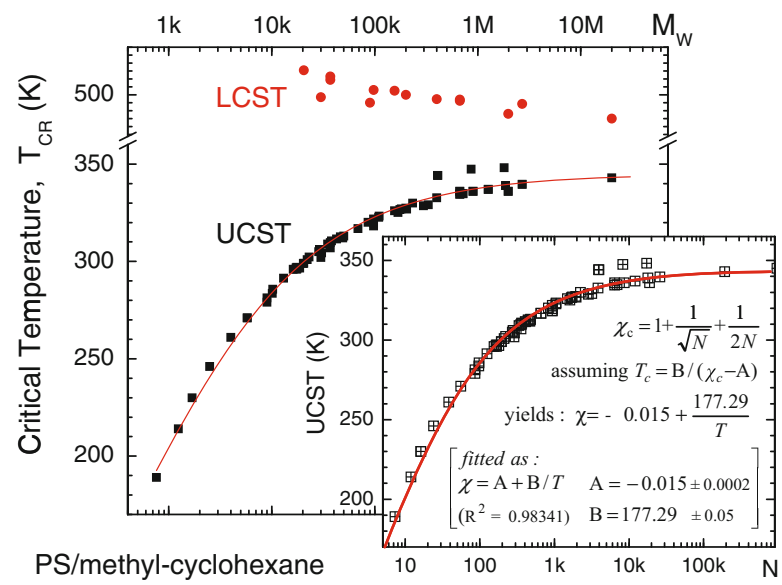

2. Polymer solutions $\left(V_{1} \gg V_{2}\right.$ or $\left.N_{2} \gg N_{2} \approx 1\right)$ are miscible when $\chi_{12}<1 / 2$, viz., $\chi_{12}<\chi_{12, c r}$ and from Eq. $2.36 \chi_{12, c r}=1 / 2+1 / \sqrt{N}+1 / 2 N \approx 1 / 2\left(\right.$ for $\left.N=N_{2}\right)$.

3. Polymer blends $\left(V_{1}\right.$ and $V_{2} \gg 1$, or $\left.N_{1} \approx N_{2} \gg 1\right)$ are miscible when $\chi_{12}<0$, viz., $\chi_{12}<\chi_{12, c r}$ and from Eq. $2.36 \chi_{12, c r}=2 / N \approx 0$ (for $N=N_{1}=N_{2}$ ). Originally, after Hildebrand, the parameter $\chi_{12}$ was assumed to have a single, characteristic value for a given mixture. However, it was soon found that even for polymer solutions $\chi_{12}$ is a complex function of many independent variables, viz., concentration, temperature, pressure, molecular weight, molecular weight distribution, etc. For calculating isobaric phase diagram, the influence of the first two variables should be expressed as (Koningsveld 1967) $\chi_{12}=\sum_{j=0}^{j \leq 2} a_{j} \phi_{2}^{j}$ with $a_{j}=\sum_{k=1}^{m \leq 2} a_{j k} T^{k}$. Thus, at constant pressure, it takes nine parameters to describe variation of $\chi_{12}$ with concentration and temperature.

In a first approximation, the temperature dependence of $\chi_{12}$ can be simplified by keeping only one temperature term, i.e., $\chi_{12}=A+B / T$, which for polymer solutions most often gives a rather good estimation. For example, in Fig. 2.6, we fitted tabulated UCST's PS/methyl-cyclohexane solutions from 71 different PS $M_{w}$ 's from 22 different studies, obtaining $\chi_{12}=-0.015+117.29 / T$ with a pretty good accuracy across multiple works and experiments. For polymer blends and for less demanding thermodynamic calculations, the concentration dependence must also be included, and most often $\chi_{12}$ is simplified by keeping one composition term and one temperature term. Thus, to express conditions of miscibility in PS blends with poly(styrene-co-4-bromostyrene), the binary interaction parameter per mer of styrene (when $T$ within the 440-500 K region) was expressed as $\chi_{12} / N=-0.02208+$ $20 / T-0.01482 \phi_{2}$ (Strobl et al. 1986). Similarly, for PS blends, the following relation was proposed (Ahn et al. 1997a, b): $B_{12}\left(\phi_{2}, T\right)=B_{0}+B_{1} \phi_{2}+B_{2} T / 1,000$ where experimental/fitted or calculated values can be used for the $B_{i}$ parameters. However, in polymer blends, there is typically a nonnegligible concentration dependence of the binary interaction parameter, i.e., $\chi=\chi(T, \phi)$, and in order to model blends by $\mathrm{FH}$ a relation of the form $\chi(T, \phi)=D(T) \cdot B(\phi)$ is used (the 
separation of variables is for simplifying the fittings to experimental data series). For example, for PS/PVME, setting

$$
\chi(T, \phi)=(1-0.4 \phi)(0.02215-8.0 / T)
$$

provided reasonable prediction of the phase behavior across various $M_{w}$ 's (Qian et al. 1991); more accurate prediction of the spinodals, for the same PS/PVME blends, necessitated the concentration term to be expanded to $\left(1-1.5 \phi+0.815 \phi^{2}\right)$ and the $T$ term to be adjusted for each polystyrene $M_{w}$ (Qian et al. 1991):

$$
\begin{aligned}
& \chi(T, \phi)=\left(1-1.5 \phi+0.815 \phi^{2}\right)(0.02754-9.0 / T) \text { low } M_{W} \\
& \chi(T, \phi)=\left(1-1.5 \phi+0.815 \phi^{2}\right)(0.0436-18.0 / T) \text { medium } M_{W} \\
& \chi(T, \phi)=\left(1-1.5 \phi+0.815 \phi^{2}\right)(0.00644-2.5 / T) \text { high } M_{W}
\end{aligned}
$$

The above compare well with results from a prior experimental approach, which employed SANS to map out the spinodal for $d$-PS/PVME, albeit parameterized using different functionals, with a linear $\phi$ dependence for both the $\chi_{H} \propto 1 / T$ and the $\chi_{S}$ terms (Schwahn et al. 1987), which are based on a EoS model.

By redefining the $T$ dependence of $\chi(T)$, e.g., to include $1 / T^{2}$ or $\ln T$ terms, one can use the FH equation to predict other type of phase diagrams, such as LCST, closed-loop, chimney, etc. (Qian et al. 1991; Eitouni and Balsara 2007). In fact, a number of reasonably accurate, within the applicable $T$ range, $\mathrm{FH}$ model $\chi(T)$ relations exist in literature for a number of polymer blends (often including a second $T$ term (i.e., $C / T^{2}$ ), see Table 2.8).

More generally, the interaction parameter dependencies on $T$ and $\phi$ can be written as

$$
\begin{aligned}
& \chi_{12}\left(\phi_{2}, T, M_{1}, M_{2}, \ldots\right)=\chi_{H}\left(\phi_{2}, M_{1}, M_{2}, \ldots\right)+\chi_{S}\left(\phi_{2}, M_{1}, M_{2}, \ldots\right) / T \\
& B_{12}\left(\phi_{2}, T, M_{1}, M_{2}, \ldots\right)=B_{H}\left(\phi_{2}, M_{1}, M_{2}, \ldots\right) T+B_{S}\left(\phi_{2}, M_{1}, M_{2}, \ldots\right)
\end{aligned}
$$

In this notation, the $T$ dependence is explicitly provided using the standard second virial coefficient functionality, i.e., $\chi \propto 1 / T$, and $\chi_{12}$ is now expressed in terms of enthalpic and entropic parts, i.e., $\chi_{H}$ and $\chi_{S}$, respectively, each being a function of concentration, molecular weight of both polymers, and other independent variables. In other words, Eq. 2.38 attempts to account for nonrandom mixing, i.e., contribution of the non-combinatorial entropy to the interactions. This idea is particularly important for polymer blends. Here, the miscibility mainly originates from strong interactions that are expected to cause changes of intersegmental orientation, hence nonrandom mixing that entails strong entropic effects. Table 2.7 gives a few example values of Eq. 2.38 parameters for selected, simple polymer blends.

As one would expect from the definition of $\chi_{12}$ (cf. viewed as the excess enthalpy/interaction between monomers in a mixed pair vs. in their single phase) 
Table 2.7 Enthalpic, $\chi_{H}$, and entropic, $\chi_{S}$, contributions to the Flory-Huggins binary interaction parameter, $\chi_{12}$, see Eq. 2.38

\begin{tabular}{llllll}
\hline Polymer- 1 & Polymer-2 & $\phi_{2}$ & $\chi_{H} \times 10^{4}$ & $\chi_{S}(\mathrm{~K})$ & Reference \\
\hline PE, $N=2,538$ & $d$-PE, $N=2,464$ & 0.221 & -0.012 & 0.132 & 1 \\
\hline PE, $N=3,308$ & $d$-PE, $N=3,275$ & 0.087 & 2.761 & 0.069 & 1 \\
\hline PE, $N=3,308$ & $d$-PE, $N=3,275$ & 0.457 & 0.242 & 0.089 & 1 \\
\hline PE, $N=4,598$ & $d$-PE, $N=4,148$ & 0.044 & 1.628 & 0.325 & 1 \\
\hline PE, $N=4,598$ & $d$-PE, $N=4,148$ & 0.087 & 0.759 & 0.138 & 1 \\
\hline PE, $N=4,598$ & $d$-PE, $N=4,148$ & 0.131 & 0.808 & 0.100 & 1 \\
\hline PE, $N=4,598$ & $d$-PE, $N=4,148$ & 0.221 & -0.907 & 0.139 & 1 \\
\hline PE, $N=4,598$ & $d$-PE, $N=4,148$ & 0.457 & -0.843 & 0.127 & 1 \\
\hline PE, $N=4,598$ & $d$-PE, $N=4,148$ & 0.708 & -0.859 & 0.133 & 1 \\
\hline PS, $N=15,400$ & $d$-PS, $N=8,700$ & 0.500 & -2.900 & 0.200 & 1 \\
\hline PpMS, $N=498$ & $d$-PS, $N=291$ & 0.250 & -0.011 & 70 & 2 \\
\hline PpMS, $N=498$ & $d$-PS, $N=291$ & 0.500 & -0.0081 & 57 & 2 \\
\hline PpMS, $N=498$ & $d$-PS, $N=291$ & 0.749 & -0.0081 & 61 & 2 \\
\hline PpMS, $N=1,108$ & $d$-PS, $N=291$ & 0.249 & -0.0119 & 72 & 2 \\
PpMS, $N=835$ & $d$-PS, $N=3,123$ & 0.5 & -2 & 2.16 & 3
\end{tabular}

Notes: $N$ is the degree of polymerization, d-PE deuterated PE, d-PS deuterated PS, PpMS poly( $p$-methylstyrene)

References: 1. Londono et al. 1994; 2. Londono and Wignall 1997; 3. Jung and Fischer 1988

there is marked independence of $\chi_{12}$ on the molecular weight of the polymers, $N$. However, large variability of $\chi_{12}$ has been observed with the concentration of the polymer blends, showing often linear and, in some cases, quadratic dependencies of $\chi_{12}$ on $\phi$ (e.g., Han et al. 1988; Krishnamoorti et al. 1994a) and on $T$ (e.g., Eitouni and Balsara 2007, and references therein).

A compilation of $\chi_{12}(T)$ parameters, spanning numerous polymer blends, showed that it is often necessary that a second $T$ term is added to Eq. 2.38 to obtain satisfactory accuracy; thus, the following the empirical dependence of $\chi_{12}$ on $T$

$$
\chi_{12}(T)=A+B / T+C / T^{2}
$$

is obtained, and a compilation of $\chi_{12}(T)$ such functions for about 120 polymer blends can be found in the second edition of "Physical Properties of Polymers Handbook" (Eitouni and Balsara 2007) (see also Table 2.8). Most of these data were obtained by applying RPA (random phase approximation) to small-angle neutron scattering (SANS) profiles measured from homogenous homopolymer blends. This approach was pioneered by Hadziioannou and Stein (1983, 1984), Murray et al. (1985), and Herkt-Maetzky and Schelten (1983). In these cases, one needs to consider also the dependence of $\chi_{12}$ on the deuteration effects of polymers, whereby there can be appreciable changes in $\chi$ depending on deuteration (see Tables 2.7, 2.8); indicatively, appreciable interaction parameters can manifest even between the hydrogenated and deuterated homologues of the same polymer, 
Table 2.8 Temperature dependence of $\chi_{12}(T)$ parameterized as $\chi_{12}(T)=A+B / T+C / T^{2}$ (Eq. 2.39), including the temperature range of parameter validity. Selected polystyrene (PS) and polypropylene (PP) blends are tabulated here. A much more extensive compilation can be found in (Eitouni and Balsara 2007)

\begin{tabular}{|c|c|c|c|c|c|}
\hline Polymer-1 & Polymer-2 & $A$ & $B(\mathrm{~K})$ & $C\left(\mathrm{~K}^{2}\right)$ & $T$ range $\left({ }^{\circ} \mathrm{C}\right)$ \\
\hline$d$-PS & PMMA & 0.0174 & 2.39 & - & $120-180$ \\
\hline PS & $d$-PMMA & 0.0180 & 1.96 & - & $170-210$ \\
\hline PS & PMMA & 0.0129 & 1.96 & - & $100-200$ \\
\hline$d$-PS & $d$-PMMA & 0.0154 & 1.96 & - & $130-210$ \\
\hline$d$-PS & PVME & 0.0973 & -41.6 & - & $60-150$ \\
\hline PS & PVME & 0.103 & -43.0 & - & $60-150$ \\
\hline PS & $d$-PXE & 0.058 & -37.7 & - & $100-280$ \\
\hline$d$-PS & PXE & 0.059 & -32.5 & - & $180-330$ \\
\hline PS & $\mathrm{PI}_{(7)}$ & 0.00785 & 17.6 & - & $100-180$ \\
\hline$d$-PS & PCHA & 0.067 & -35 & - & $120-155$ \\
\hline PS & P2VP & 0.018 & 35 & - & $155-230$ \\
\hline$d$-PS & PPMA & 0.0515 & -27.2 & 5127 & $80-130$ \\
\hline$d$-PS & PBMA & 0.107 & -60.4 & 9807 & $20-130$ \\
\hline PS & P4MS & 0.0046 & 3.2 & - & $160-230$ \\
\hline $\mathrm{PP}$ & $d_{3}-\mathrm{SPB}_{(97)}$ & 0.00454 & -4.71 & 1364 & $30-130$ \\
\hline$d_{4}-\mathrm{PP}$ & $\mathrm{SPB}_{(97)}$ & 0.00244 & -3.27 & 1051 & $30-130$ \\
\hline $\mathrm{PP}$ & $\mathrm{SPB}_{(97)}$ & 0.00349 & -3.99 & 1208 & $30-130$ \\
\hline $\mathrm{PP}$ & $d_{3}-\mathrm{SPB}_{(78)}$ & 0.00747 & -6.38 & 1426 & $50-170$ \\
\hline$d_{4}-\mathrm{PP}$ & $\mathrm{SPB}_{(78)}$ & 0.00381 & -3.50 & 895 & $50-170$ \\
\hline $\mathrm{PP}$ & $\mathrm{SPB}_{(78)}$ & 0.00564 & -4.94 & 1161 & $50-170$ \\
\hline $\mathrm{PP}$ & $d_{5}-\mathrm{SPI}_{(7)}$ & 0.00302 & 4.59 & 944 & $30-170$ \\
\hline$d_{4}-\mathrm{PP}$ & $\operatorname{SPI}_{(7)}$ & 0.00392 & 5.39 & 969 & $30-170$ \\
\hline $\mathrm{PP}$ & $\operatorname{SPI}_{(7)}$ & 0.00347 & 4.99 & 957 & $30-170$ \\
\hline HHPP & $d_{2}-\mathrm{SPB}_{(78)}$ & 0.00153 & 1.24 & - & $110-170$ \\
\hline$d_{4}$-HHPP & $\mathrm{SPB}_{(78)}$ & 0.00220 & 1.40 & - & $30-170$ \\
\hline HHPP & $\mathrm{SPB}_{(78)}$ & 0.00187 & 1.32 & - & $110-170$ \\
\hline HHPP & $d_{3}-\mathrm{SPB}_{(66)}$ & 0.00716 & -6.17 & 1338 & $30-170$ \\
\hline$d_{4}$-HHPP & $\mathrm{SPB}_{(66)}$ & 0.00675 & -5.84 & 1280 & $30-170$ \\
\hline HHPP & $\mathrm{SPB}_{(66)}$ & 0.00696 & -6.01 & 1309 & $30-170$ \\
\hline HHPP & $d_{3}-\mathrm{PEB}$ & 0.00127 & -0.96 & 282 & $30-170$ \\
\hline$d_{4}$-HHPP & PEB & 0.00243 & -1.86 & 457 & $30-170$ \\
\hline HHPP & PEB & 0.00185 & -1.41 & 370 & $30-170$ \\
\hline HHPP & $d_{5}-\mathrm{SPI}_{(7)}$ & 0.00806 & -5.71 & 1046 & $30-170$ \\
\hline$d_{4}$-HHPP & $\mathrm{SPI}_{(50)}$ & 0.00220 & 1.24 & - & $30-170$ \\
\hline HHPP & $d_{5}-\mathrm{SPI}_{(50)}$ & 0.00174 & 1.29 & - & $50-170$ \\
\hline HHPP & $\operatorname{SPI}_{(50)}$ & 0.00197 & 1.27 & - & $50-170$ \\
\hline HHPP & $d_{4}-\mathrm{PP}$ & 0.00427 & 2.13 & - & $30-130$ \\
\hline
\end{tabular}


Table 2.8 (continued)

\begin{tabular}{lllccc}
\hline Polymer-1 & Polymer-2 & $A$ & $B(\mathrm{~K})$ & $C\left(\mathrm{~K}^{2}\right)$ & $T$ range $\left({ }^{\circ} \mathrm{C}\right)$ \\
\hline$d_{4}$-HHPP & PP & 0.00301 & 1.54 & - & $30-130$ \\
HHPP & PP & 0.00364 & 1.84 & - & $30-130$ \\
\hline$d_{4}$-HHPP & PIB & 0.0180 & -7.74 & - & $30-170$ \\
\hline
\end{tabular}

Notes: The values of $A, B$, and $C$ and thus of $\chi$ are based on a reference volume $V_{\text {ref }}=0.1 \mathrm{~nm}^{3}$ Polymer notation: A $d$ - label preceding the polymer acronym indicates a per-deuterated polymer; partially deuterated polymers are labeled as $d_{3^{-}}, d_{4^{-}}$, etc., for selective deuteration of 3 , 4 , etc., hydrogens. Numbers in subscripted parentheses after the polymer name indicate the primary comonomer fraction, e.g., $\mathrm{SPB}_{(66)}$ is a saturated polybutadiene with $66 \mathrm{~mol} \%$ butadiene

Polymer acronyms: $P 2 V P$ poly(2-vinyl pyridine), $P 4 M S$ poly(4-methylstyrene), $P B M A$ poly( $n$-butyl methacrylate), PCHA poly(cyclohexyl acrylate), $P E B$ poly(ethyl butylene), PIB polyisobutylene, $P I$ polyisoprene, $P M M A$ poly(methyl methacrylate), $P P M A$ poly ( $n$-pentyl methacrylate), $P P$ polypropylene, $H H P P$ head-to-head polypropylene, $P S$ polystyrene, $P V M E$ poly(vinyl methyl ether), $P X E$ poly(2,6-dimethyl-1,4-phenylene oxide), $S P B$ saturated polybutadiene, $S P I$ saturated polyisoprene

Fig. 2.7 The binary interaction parameter for blends of PS with $d$-PS at $T=433 \mathrm{~K}$ and for PE with $d$-PE at $T=443 \mathrm{~K}$ (Londono et al. 1994)

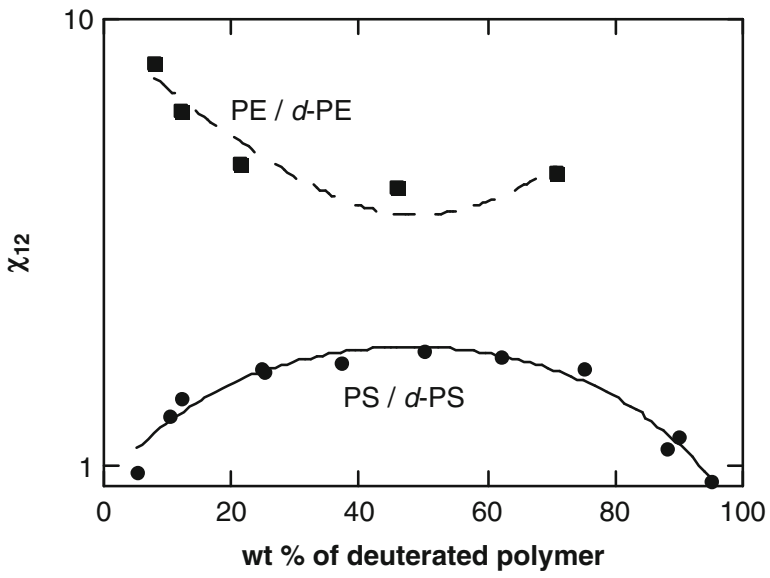

oftentimes leading to phase separation of such, especially at large molecular weights. For example, in Fig. 2.7, the experimental values of this parameter are shown for blends of a hydrogenated polymer with its deuterated homologue, i.e., PE with $d$-PE at $T=443 \mathrm{~K}$ and PS with $d$-PS at $T=433 \mathrm{~K}$ (Londono et al. 1994). The data can be described in terms of Eq. 2.38. A formal analysis of these data was published by Bidkar and Sanchez (1995).

Even at a superficial view, it is rather obvious that the FH theory has limitations, even when all its restrictive assumptions (weak interactions, entropy-independent enthalpy, etc.) are satisfied. For example, the $\chi$ for polymers, as defined by FH $(\chi=z \Delta \varepsilon / k T ; z$ is coordination number and $\Delta \varepsilon$ the excess enthalpy of interaction for one mixed pair) allows for $z$ solvent neighbors around each monomer, neglecting that 
Table 2.9 Structural parameters for model polyolefins (Dudowicz and Freed 1996a, b)

\begin{tabular}{lllll}
\hline Polyolefin & & $\mathrm{r}$ & $\mathrm{p}$ & $\mathrm{q}$ \\
\hline PE & Polyethylene & 1 & 0 & 0 \\
\hline PEP & Poly(ethylene propylene) & 1.2 & 0.2 & 0.25 \\
\hline PPE & Poly-1-pentene [poly(propyl ethylene)] & 1.2 & 0.2 & 0.25 \\
\hline PEE & Poly-1-butene [poly(ethyl ethylene)] & 1.25 & 0.25 & 0.33 \\
\hline PP & Polypropylene & 1.33 & 0.33 & 0.5 \\
\hline P2B & Poly-2-butene & 1.5 & 0.5 & 1 \\
\hline PDMB & Poly(4,4-dimethyl 1-butene) & 1.67 & 0.5 & 1 \\
\hline PIB & Polyisobutylene & 1.75 & 0.5 & 1 \\
\hline
\end{tabular}

there are always (at least) two other monomers of either side of an internal monomer; this leads to a gross overestimation of the nearest neighbor heterocontacts, which, however, can be addressed by a simple correction, replacing $z$ by $z-2$ (Guggenheim 1944, 1952). A number of other extensions of the FH theory also address the type and geometry of monomer, the stiffness of the backbone, the existence of unsaturated carbons, etc.; the reader is referred to a recent review (Freed and Dudowicz 2005, and references cited therein). More arguments along these same lines led to correction terms or extensions in the FH theory that can address, beyond chain connectivity, also monomer size, monomer geometry including pending groups, restricted bond rotations, etc., details that can become very important when comparing to sensitive experimental data, such as SANS measurements of $\chi$. The details of such corrections go beyond the scope of this chapter; the interested reader is again referred to Freed and Dudowicz (2005); in most cases, such extensions retain the FH equation for the free energy of mixing, Eq. 2.35, and redefine the $\chi^{\prime}$ parameter as an appropriate function, rather than a system-specific constant. Thus, such corrections lead to a binary interaction parameters with functionals such as

$$
\chi^{\prime}(\phi, T)=a+\frac{b+c \phi}{T} \quad \text { or } \quad \chi^{\prime}(\phi, T)=a^{\prime}+\frac{b^{\prime}}{T}+c^{\prime} \phi
$$

where $a, b$, and $c$ are corrections due to monomer geometry, packing (see also Table 2.9ff.), and other $\left(b^{\prime}, c^{\prime}\right)$ considerations, often rather involved in their definition (e.g., Eq. 11a vs. Eq. 2.10 in Freed and Dudowicz 2005). Finally, another drawback of the FH theory is the assumption of a fully occupied lattice, i.e., the assumption that all space is occupied by units, either solvent molecules or polymeric segments, of equal and constant size. As a consequence, the free volume contributions are largely neglected. It was pointed out that dissolution of polymer is associated with volume changes (Maron 1959), leading to a modification of the FH theory extended to account for ternary systems comprising of polydispersed polymers (Utracki 1962).

\subsubsection{Equation of State Theories}

Starting in the early 1960 s considerable effort was made to develop what become known as the equation of state theories (Flory et al. 1964; Eichinger and Flory 1968; 
Simha and Somcynsky 1969; Patterson 1969, 1982; Patterson and Robard 1978; Sanchez and Lacombe 1976, 1977; Sanchez 1983, 1984). The equation of state (EoS) theories of mixtures are based on the principles discussed in Sect. 2.4.1. Formally, the computation of the partition function for a single component or for a mixture of components is similar, yielding the Helmholtz free energy of mixing.

Different EoS use different measures of the binary interactions between components. For example, in Flory's extension of the FH approach, the binary interaction parameter, $\chi_{12}$, is still present (Flory 1970). In S-L EoS (see Eq. 2.16), the characteristic pressures for the mixture of species are assumed to be pairwise additive:

$$
P^{*}=\sum_{i} \phi_{i} P_{i}^{*}-\sum_{i<j} \sum_{j} \phi_{i} \phi_{j} \Delta P^{*}
$$

For such systems, the difference of the interaction energy density, $\Delta P^{*}$, has been considered a measure of the binary interactions between polymeric segments, proportional to either $\chi_{12}$ or $B$ (Sanchez 1989).

The most successful statistical thermodynamic theory of liquids is that proposed by Simha and Somcynsky, S-S (Simha and Somcynsky 1969; Jain and Simha 1980, 1981, 1982, 1984). From the corresponding partition function, the Helmholtz free energy and then the pressure were computed using the general derivatives, Eq. 2.5. For a single-component system, the S-S yielded $P V T$ relationships (Eq. 2.19). Initially, the theory has been used to compute $P V T$ behavior of homopolymers. Later, it was extended to full thermodynamic description of multicomponent systems, viz., thermodynamics of mixtures, gas-liquid and liquid-liquid phase equilibria, etc. Thus, using the derived expression for the free energy of neat components, the molar Helmholtz free energy was derived for a binary mixture (Jain and Simha 1980; 1984; Stroeks and Nies 1988):

$$
\begin{aligned}
\frac{F_{m}}{R T}= & x_{1} \ln x_{1}+x_{2} \ln x_{2}+\frac{\langle s\rangle}{y}(1-y) \ln (1-y)-(\langle s\rangle-1) \ln \frac{z-1}{e} \\
& -\langle c\rangle\left[\ln \frac{\left\langle V^{*}\right\rangle(1-\eta)^{3}}{Q}-\left(\frac{y Q^{2}}{2 T^{*}}\right)\left(A Q^{2}-2 B\right)\right] \\
& -\frac{3}{2}\left[x_{1} c_{1} \ln \left(2 \pi \frac{\left\langle M_{o 1}\right\rangle}{\left(N_{A} h\right)^{2}}\right)+x_{2} c_{2} \ln \left(2 \pi \frac{\left\langle M_{o 2}\right\rangle}{\left(N_{A} h\right)^{2}}\right)\right]
\end{aligned}
$$

where $N_{A}$ and $h$ are Avogadro's and Planck's constants, respectively and the nomenclature follows the typical variables of the S-S EoS, such as $y$ the fraction of occupied lattice cells; $Q$ the dimensionless quantity $1 /\left(y V^{*}\right) ; \eta$ the dimensionless quantity $2^{-1 / 6} y Q^{1 / 3} ; s_{i}$ the number of segments per chain of molar mass $M_{i} ; M_{o i}$ the segmental molar mass, $M_{o i}=M_{i} / s_{i}$; as well as the three principal reducing variables $P_{i}^{*}\left(=z q_{i} \epsilon_{i i}^{*} / s_{i} v_{i i}^{*}\right), T_{i}^{*}\left(=z q_{i} \epsilon_{i i}^{*} / R c_{i}\right)$, and $V_{i}^{*}\left(=v_{i i}^{*} / M_{o i}\right)$, which relate to the component's maximum molar intermolecular attraction energy per segment $\left(\epsilon_{i i}^{*}\right)$ and the number of intermolecular contacts $\left(q_{i} z=s_{i}(z-2)+2\right)$, with the subscripts $i$, in all 
Fig. 2.8 The binary interaction parameters $S_{\varepsilon}$ and $S_{v}$ of the Simha-Somcynsky (S-S) EoS for PS/PPE blends ( $T$ range, $T_{g}$ to $330^{\circ} \mathrm{C}$; $P$ range, $0-180 \mathrm{MPa})(\mathrm{Jain}$ et al. 1982)

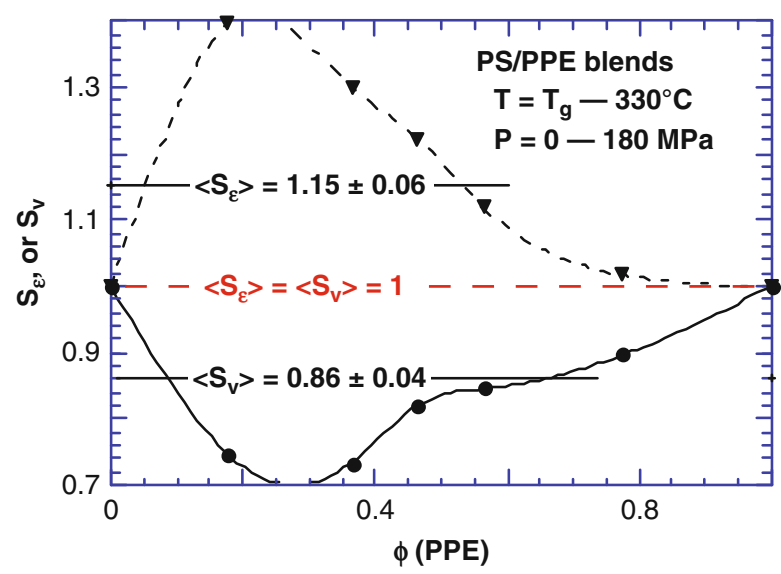

cases, indicating the value for component $i$. And the mixture variables, the values in angle brackets, \langle\rangle , which are compositional averages based on the components' properties and their molar fractions, $x_{i}$, in the mixture:

$$
\begin{aligned}
& \langle s\rangle=x_{1} s_{1}+x_{2} s_{2} \quad \text { and }\langle c\rangle=x_{1} c_{1}+x_{2} c_{2} \\
& \left\langle M_{o}\right\rangle=\left(x_{1} s_{1} M_{o 1}+x_{2} s_{2} M_{o 2}\right) /\left(x_{1} s_{1}+x_{2} s_{2}\right) \\
& \left\langle\epsilon^{*}\right\rangle\left\langle v^{*}\right\rangle^{k}=X_{1}^{2} \epsilon_{11}^{*} v_{11}^{* k}+X_{2}^{2} \epsilon_{22}^{*} v_{22}^{* k}+2 X_{1} X_{2} \epsilon_{12}^{*} v_{12}^{* k} \quad(k=2,4) \\
& \text { where } X_{1}=1-X_{2}=x_{1}\left[s_{1}(z-2)+2\right] /\langle q z\rangle
\end{aligned}
$$

In Eq. 2.43, there are two cross-interaction parameters, quantified through weight-averaged mixing rules, that characterize the binary system:

1. The interaction energy, $\epsilon_{12}^{*}=S_{\epsilon} \sqrt{\epsilon_{11}^{*} \epsilon_{22}^{*}}$

2. The repulsion volume, $v_{12}^{*}=S_{v}\left[\left(v_{11}^{*}{ }^{1 / 3}+v_{22}^{*}{ }^{1 / 3}\right) / 2\right]^{3}$

For nearly athermal systems, the proportionality factors, $S_{\varepsilon}$ and $S_{v}$, are taken as equal to 1 . Thus, for the systems without strong interactions, the binary parameters are well approximated by the geometric and algebraic averages. For example, for PS/PVME blends, the assumption $S_{\varepsilon}=S_{v}=1$ resulted in $0.1 \%$ deviation for the experimental values of the cross-parameters (Xie et al. 1992; Xie and Simha, 1997, "private communication"). In contrast, it is to be expected that for systems with strong intermolecular interactions such mixture rules may fail and experimental values for the cross-factors may have to be found. However, least squares fit of Eqs. 2.42 and 2.43 to experimental values of $\mathrm{CO}_{2}$ miscibilities in PS (in a wide range of $P$ and $T$ ) yielded values for $S_{\varepsilon}$ and $S_{v}$ close to 1 (Xie et al. 1997).

The S-S equations were used for the description of $P V T$ dependencies for PS, PPE, and their blends (Jain et al. 1982). The data are presented in Fig. 2.8, where the best-fit values for each composition: $S_{\varepsilon}(P, T, \phi)$ and $S_{v}(P, T, \phi)$ are plotted versus $\mathrm{PPE}$ volume fraction, $\phi(\mathrm{PPE})$. The plot shows concentration, a nontypical dependence for these two parameters, i.e., a behavior not previously observed for mixtures of solvents or for polymer solutions; the variation is not large - the 
averages of $S_{\varepsilon}$ and $S_{v}$ are, respectively, $1.15 \pm 0.06$ and $0.86 \pm 0.04$. From a theoretical point of view, it is highly satisfying that these values are not far from unity. Furthermore, the larger (than 1) value of $S_{\varepsilon}$ and the smaller (than 1) value of $S_{v}$ indicate the presence of specific interactions between PS and PPE, larger than the geometric average energetic interactions, and a contraction of the binary volume contribution, smaller than the algebraic mean. It should be stressed that, as shown by the original authors, the $S_{\varepsilon}$ and $S_{v}$ values provided excellent description of the $P V T$ dependencies over the full range of variables. The observed compositional variability may, thus, be caused by inadequacies of the theory, or the computational method, or the experimental data. Analysis of these and other blends by means of the S-S EoS is continuously being pursued.

\subsubsection{Gas-Lattice Model}

The gas-lattice model considers liquids to be a mixture of randomly distributed occupied and vacant sites. $P$ and $T$ can change the concentration of holes, but not their size. A molecule may occupy $m$ sites. Binary liquid mixtures are treated as ternary systems of two liquids (subscripts " 1 " and " 2 ") with holes (subscript " 0 "). The derived equations were used to describe the vapor-liquid equilibrium of $n$-alkanes; they also predicted well the phase behavior of $n$-alkanes/PE systems. The gas-lattice model gives the non-combinatorial Helmotz free energy of mixing expressed in terms of composition and binary interaction parameters, quantified through interaction energies per unit contact area (Kleintjens 1983; Nies et al. 1983):

$$
\begin{aligned}
\Delta F_{m} / N_{\phi} k T= & \phi_{0} \ln \phi_{0}+\left(\phi_{1} / m_{1}\right) \ln \phi_{1}+\left(\phi_{2} / m_{2}\right) \ln \phi_{2}+ \\
& {\left[\alpha_{11}+g_{11}^{L}\left(1-\gamma_{1}\right) /\left(1-\gamma_{1} \phi_{1}-\gamma_{2} \phi_{2}\right)\right] \phi_{0} \phi_{1}+} \\
& {\left[\alpha_{22}+g_{22}^{L}\left(1-\gamma_{2}\right) /\left(1-\gamma_{1} \phi_{1}-\gamma_{2} \phi_{2}\right)\right] \phi_{0} \phi_{2}+} \\
& {\left[\alpha_{12}+g_{12}^{L}\left(1-\gamma_{1}\right) /\left(1-\gamma_{1} \phi_{1}-\gamma_{2} \phi_{2}\right)\right] \phi_{1} \phi_{2} }
\end{aligned}
$$

with $N_{\phi}=n_{0}+n_{1} m_{1}+n_{2} m_{2}$ and $\gamma_{i}=1-\sigma_{i} / \sigma_{0}$; the superscript $L$ is used to indicate that the relation was derived from the gas-lattice model; the parameter $s_{i}$ is the interacting surface area of species " $i$ "; the binary interaction parameters include two terms, as usual, the $\alpha_{i j}$ empirical entropy corrections and the $g_{i j}$ temperature dependencies $\left(g_{i j}=-w_{i j} / 2 \sigma_{0} / k T\right.$, with $w_{i j}$ is the interaction energy per unit contact surface area involved in $i-j$ contact, $i=j$ corresponds to same-species interactions). This approach is rather versatile, and, among other, it has been extended and adapted to describe the empirical parameters through a molecular basis (Koningsveld et al. 1987), as well as orientation-dependent interactions (Besseling and Scheutjens 1994). According to the gas-lattice theory, four factors determine the polymer/ polymer miscibility (Koningsveld et al. 1982: Koningsveld 1986):

1. Interacting surface areas of segments

2. Coil dimensions (dependent on $T, \phi$, and $M_{W}$ )

3. Molecular weight polydispersity

4. Free volume fraction

In an effort to address some of the FH and gas-lattice models simplifications, the lattice cluster theory (LCT) was developed (Dudowicz et al. 1991; Dudowicz 
and Freed 1991, 1993, 1995, 1996a, b; Freed and Dudowicz 1995; 1996a, b; 2005). LCT is a mean-field lattice-based model, but in comparison to FH theory, it incorporates two modifications: (1) It includes local packing and interactions, and (2) it distinguishes different structures of the monomeric units, allowing the monomeric units to occupy different adjacent sites, as dictated by their structure (e.g., pending side groups). The theory represents polymer chains as strings of beads, called united atoms, freely jointed by flexible bonds. For example, it considers $\mathrm{CH}_{\mathrm{x}}$ $(\mathrm{x}=0,1,2$, or 3$)$ as a single bead (or group) that occupies one lattice site. LCT also incorporates vacant sites (free volume) and uses the nonrandom mixing principle. Thus, the free energy of mixing is given by

$$
\frac{\Delta F_{m}}{N k T}=\sum_{i} \frac{\phi_{i}}{M_{i}} \ln \phi_{i}+\phi_{v} \ln \phi_{v}+\sum_{i} g_{i}\left(\phi_{i}\right) \phi_{i} \phi_{v}+\sum_{i \neq j} g_{i j}\left(\phi_{i}, \phi_{j}\right) \phi_{i} \phi_{j}
$$

The first two terms of Eq. 2.45 are the combinatorial entropy contributions, one for each species $i$ and one, the second term, for the free volume contribution to the entropy of mixing (where the subscript $v$ indicates the free volume fraction). The third term represents the non-combinatorial contribution $\left(g_{i}\left(\phi_{i}\right)\right.$ is the non-combinatorial energy of a molten state of polymer $i$ having the free volume fraction $\phi_{v}$ ). The fourth term represents the energetic contribution originating from interaction between unlike species, $i \neq j$. Here, $g_{i j}\left(\phi_{i}, \phi_{j}\right)$ is the interaction term expressed as a polynomial with coefficients that depend on the structure of the polymer chains; these coefficients are computed as double expansions in $1 / z$ ( $z$ is the lattice coordination number) and $\varepsilon_{i j} / k T$ ( $\varepsilon_{i j}$ is the van der Waals interaction energies between groups $i$ and $j$ ). Through curve fitting to experimental data, it has been shown that the binary interaction parameter, Flory-Huggins' $\chi_{12}$ or $g_{12}$ above, is a thermodynamic function of such independent variables as $T, P, \phi_{i}$, molecular weight, and others. LCT shows that the binary interaction parameter has strong sensitivity also to composition, monomeric structure, and local correlation. The theory was quite successful describing observed dependencies for PS/PVME blends using four parameters: $\varepsilon_{\mathrm{S} / \mathrm{S}}, \varepsilon_{\mathrm{VME} / \mathrm{VME}}, \varepsilon_{\mathrm{S} / \mathrm{VME}}$, and the cell volume. Except for the heterocontact parameter $\varepsilon_{\mathrm{S} / \mathrm{VME}}$ (which must be determined by a fitting procedure), the values of the other three parameters are determined from the $P V T$ dependencies of neat resins and are not adjustable in the blend phase calculations.

LCT, originally developed for di-block copolymers, was found to be particularly useful to explain miscibility of polyolefin blends, where the two resins differ in the type and size of short-chain branching. These polymers comprise of structural units with two carbons in the main chain (backbone), i.e., polyethylene, $\mathrm{PE}=\left[\mathrm{CH}_{2}-\mathrm{CH}_{2}\right]_{\text {n }}$; polypropylene, $\mathrm{PP}=\left[\mathrm{CH}_{2}-\mathrm{CH}\left(\mathrm{CH}_{3}\right)\right]_{\mathrm{n}}$; poly-2-butene, $\mathrm{P} 2 \mathrm{~B}=\left[\mathrm{CH}\left(\mathrm{CH}_{3}\right)-\mathrm{CH}\right.$ $\left.\left(\mathrm{CH}_{3}\right)\right]_{\mathrm{n}}$; polyisobutylene, PIB $=\left[\mathrm{CH}_{2}-\mathrm{C}\left(\mathrm{CH}_{3}\right)_{2}\right]_{\mathrm{n}}$; poly(4,4-dimethyl 1-butene), $\mathrm{PDMB}=\left[\mathrm{CH}_{2}-\mathrm{CH}\left(\mathrm{C}_{4} \mathrm{H}_{9}\right)\right]_{\mathrm{n}}$; etc. Structural parameters (e.g., ratio of end to interior groups, number of bonds, volume of submonomer units, etc.) are used to distinguish between different monomer structures and geometries and account for differences in the blend phase behavior by redefining $\chi(\phi, T)$ (see Eq. 2.40). For example, three such 
Table 2.10 Binary interaction parameters, $\chi=\chi_{s}+\chi_{H} / T$, for model polyolefin 1:1 blends at $500 \mathrm{~K}$ (Freed and Dudowicz 1996a, b). See also Tables 2.9 and 2.8

\begin{tabular}{llllll}
\hline Blends & $T_{c}(\mathrm{~K})$ & $r$ & $1,000 \chi_{s}$ & $\chi_{H}(\mathrm{~K})$ & $100 \chi$ \\
\hline PE/PIB & 488 & 0.75 & -0.3248 & 5.8188 & 1.13 \\
\hline PEP/PIB & 477 & 0.55 & 0.4560 & 2.8443 & 0.615 \\
\hline PE/P2B & 432 & 0.5 & 1.877 & 1.6352 & 0.510 \\
\hline PP/PIB & 395 & 0.417 & 1.840 & 0.9062 & 0.354 \\
\hline PP/PE & 383 & 0.333 & 1.356 & 0.5150 & 0.229 \\
\hline PEP/P2B & 365 & 0.3 & 1.327 & 0.3259 & 0.187 \\
\hline P2B/PIB & 351 & 0.25 & 1.049 & 0.2119 & 0.135 \\
\hline PE/PEP & 340 & 0.2 & 0.6486 & 0.1249 & 0.083 \\
\hline PP/P2B & 328 & 0.167 & 0.5463 & 0.0569 & 0.061 \\
\hline PEP/PP & 311 & 0.133 & 0.3249 & 0.0424 & 0.037 \\
\hline
\end{tabular}

parameters, $r, p$, and $q$, were used for the polyolefins above (values for model polyolefin macromolecules are summarized in Table 2.9). A number of derivative models (simplified-LCT, basic-LCT, etc.) were also developed by the same scientists (Freed and Dudowicz 2005).

Miscibility is expected for blends of polyolefins having similar values of these structural parameters. In Table 2.10, examples of the computed binary interaction parameters for 1:1 composition polyolefin blends at $500 \mathrm{~K}$ are shown (Freed and Dudowicz 1996a, b). The experimental values of these parameters have also been measured (Balsara et al. 1992, 1994; Graessley et al. 1993, 1994a, b, 1995; Krishnamoorti et al. 1994a, b, 1995, 1996; Lin et al. 1996; Schipp et al. 1996; Reichart et al. 1997). Experimental data were determined using either small-angle neutron scattering (SANS), cloud-point curve determination (CPC), or PVT measurements. The experimental results will be discussed later, vide infra SANS measurements.

\subsubsection{Off-Lattice Theories}

\subsubsection{Strong Interactions Model}

For incompressible systems having strong interactions, e.g., acid-base type, the directional-specific model of segmental interactions may be used (Walker and Vause 1982; ten Brinke and Karasz 1984). By appropriate definition of $\chi(T)$, the familiar FH expression was derived for a symmetric $\left(N_{1}=N_{2}=N\right)$ blend:

$$
\begin{aligned}
& \frac{\Delta G_{m}}{R T}=\frac{\phi_{1}}{N} \ln \phi_{1}+\frac{\phi_{2}}{N} \ln \phi_{2}+\chi(T) \phi_{1} \phi_{2} \\
& \text { with } \chi=\frac{U_{2}}{R T}+\ln (1-\lambda)+\ln \left(1+\frac{1}{q}\right) \text { and } \lambda=\left[1+q e^{\left(U_{1}-U_{2}\right) / R T}\right]^{-1}
\end{aligned}
$$

where $U_{1}$ and $U_{2}$ are the attractive and repulsive energies, respectively, and $q$ is the degeneracy number; the familiar FH expression was obtained by splitting the binary interaction parameter $\chi$ in an enthalpic and an entropic term: 


$$
\begin{aligned}
& \chi_{H} / z=\left[\lambda U_{1}+\left(1-\lambda U_{2}\right)\right] / R T \text { and } \\
& \chi_{S} / z=\ln (1-\lambda)+\ln (1+1 / q)-\lambda\left(U_{1}-U_{2}\right) / R T
\end{aligned}
$$

respectively, with $q$ and $\lambda$ as above. Depending on the relative magnitudes of $U_{1}$ and $U_{2}$, Eq. 2.46 predicts either UCST or LCST.

\subsubsection{Heat of Mixing Approach}

For most polymers, $N \gg 1$ and the configurational entropy of a polymer blend become vanishingly small; thus, to a very good approximation $\Delta G_{m} \approx \Delta H_{m}$ (the enthalpic effects dominate) and, hence, adiabatic calorimetry should be able to predict polymer/polymer miscibility (Cruz et al. 1979).

$$
\Delta G_{m} \approx \Delta H_{m}=B \phi_{1} \phi_{2}=\chi_{12} R T\left(V / V_{1}\right) \phi_{1} \phi_{2}
$$

After experimentally confirming the validity of this idea, the principal authors attempted to use this approach for explanation of the so-called miscibility windows (Paul and Barlow 1984). The latter term refers either to polymer/copolymer blends that show miscibility only within a limited range of copolymer compositions (e.g., Balazs et al. 1985; Fernandes et al. 1986; Goh and Lee 1987) or to blends of two copolymers having a common monomer (Shiomi et al. 1986). As earlier, in Koningsveld's treatment of $\chi_{12}$, here also the parameter $B$ has an enthalpic contribution and a non-combinatorial entropic contribution. For multicomponent systems, Eq. 2.47 can be generalized (Barlow and Paul 1987):

$$
\frac{\Delta G_{m}}{V} \approx \frac{\Delta H_{m}}{V}=\sum_{i} \sum_{j \neq i} B_{i j} \phi_{i} \phi_{j}-\sum_{k} \Psi_{k} \sum_{i} \sum_{j \neq i} B_{i j} \phi_{i}^{k} \phi_{j}^{k}
$$

where $\Psi_{k}$ is the volume fraction of polymer $k$ and the usual constraints for the component volume fractions are extended to $\sum \phi_{i} \equiv 1$ and $\sum \phi_{i}^{k} \equiv 1$. For simple systems, containing a copolymer A (mers \#1 and \#2) and either a homopolymer B (mers \#3) or a copolymer B (mers \#1 and \#3), Eq. 2.48 can be simplified to read:

$$
\frac{\Delta G_{m}}{V} \approx \frac{\Delta H_{m}}{V}=B \Psi_{1} \Psi_{2}
$$

where $B$ is now, respectively,

$$
\begin{aligned}
& B=B_{13} \phi_{1}^{\prime}+B_{23} \phi_{2}^{\prime}-B_{12} \phi_{1}^{\prime} \phi_{2}^{\prime} \text { or } \\
& B=B_{12} \phi_{2}\left(\phi_{2}^{\prime}-\phi_{3}^{\prime \prime}\right)+B_{13} \phi_{3}\left(\phi_{3}^{\prime}-\phi_{2}^{\prime \prime}\right)+B_{23} \phi_{2}^{\prime} \phi_{3}^{\prime \prime}
\end{aligned}
$$

where $\phi_{i}^{\prime}$ and $\phi_{i}^{\prime \prime}$ denote the volume fraction of $i$-type monomer in copolymers $\mathrm{A}$ and $\mathrm{B}$, respectively. Over the years, values of the parameter B for many polymer mixtures have been published. As shown in Table 2.11, similar to $\chi_{12}$, also the binary $B$ varies with composition, temperature, and other blend variables. 
Table 2.11 Binary interaction parameters: $B, \Delta P^{*}$, or $\chi_{12}$ (see earlier data in Utracki 1989). To convert $\mathrm{cal} / \mathrm{mL}$ to $\mathrm{J} / \mathrm{m}^{3}$, multiply the listed values by $4.187 \times 10^{6}$

\begin{tabular}{|c|c|c|c|c|c|}
\hline Polymer-1 & Polymer-2 & $T\left({ }^{\circ} \mathrm{C}\right)$ & $B(\mathrm{cal} / \mathrm{mL})$ & $\Delta P^{*}(\mathrm{cal} / \mathrm{mL}) \quad \chi_{12}$ & References \\
\hline PS & TMPC & 30 & -0.13 & $-0.17 \pm 0.01$ & 1 \\
\hline PS & TMPC & 300 & 0.11 & $-0.17 \pm 0.01$ & 1 \\
\hline PS & $\mathrm{P} \alpha-\mathrm{MS}$ & 50 & $0.012-0.025$ & $0.011-0.025$ & 2 \\
\hline $\mathrm{PS}_{(50)}$ & $\mathrm{P} \alpha-\mathrm{MS}$ & & $\begin{array}{l}-0.0833+ \\
0.001034 \mathrm{~T}\end{array}$ & & 2 \\
\hline PS & PMMA & 160 & 0.542 & 0.620 & 2 \\
\hline PS & PMMA & 245 & 0.464 & 0.532 & 2 \\
\hline PS & PMMA & 153 & 0.457 & 0.520 & 2 \\
\hline PS & PMMA & 250 & 0.392 & 0.455 & 2 \\
\hline PS & PMMA & 195 & $0.21 \pm 0.02$ & $0.24 \pm 0.05$ & 2 \\
\hline $\mathrm{PS}_{(50)}$ & PMMA & & $0.542+10^{-4}$ & & 2 \\
\hline $\mathrm{P} \alpha-\mathrm{MS}$ & PMMA & 150 & 0.354 & & 2 \\
\hline $\mathrm{P} \alpha-\mathrm{MS}$ & PMMA & 250 & 0.458 & & 2 \\
\hline PS & $\mathrm{PC}$ & 50 & 0.43 & 0.44 & 3 \\
\hline PS & DMPC & 50 & $0.20-0.49$ & - & 3 \\
\hline PS & TMPC & 240 & 0.036 & -0.17 & 3 \\
\hline PS & TCPC & 50 & $>0.72$ & - & 3 \\
\hline PS & PCZ & 50 & 0.28 & - & 3 \\
\hline PS & HFPC & 300 & $>14$ & $>1.6$ & 3 \\
\hline PS & $\mathrm{BCPC}$ & 50 & 1.5 & $>0.33$ & 3 \\
\hline PS & TMPC-P & 180 & 0.16 & 0.023 & 3 \\
\hline $\mathrm{P} \alpha-\mathrm{MS}$ & $\mathrm{PC}$ & 50 & $0.39-0.44$ & $0.42-0.49$ & 3 \\
\hline $\mathrm{P} \alpha-\mathrm{MS}$ & DMPC & 50 & $>0.18$ & - & 3 \\
\hline $\mathrm{P} \alpha-\mathrm{MS}$ & TMPC & 180 & 0.26 & 0.068 & 3 \\
\hline $\mathrm{P} \alpha-\mathrm{MS}$ & TCPC & 300 & $>0.31$ & - & 3 \\
\hline $\mathrm{P} \alpha-\mathrm{MS}$ & PCZ & 200 & $>0.24$ & - & 3 \\
\hline $\mathrm{P} \alpha-\mathrm{MS}$ & HFPC & 50 & $0.22-0.72$ & $0.12-1.4$ & 3 \\
\hline $\mathrm{P} \alpha-\mathrm{MS}$ & $\mathrm{BCPC}$ & 300 & $>0.33$ & $>0.44$ & 3 \\
\hline $\mathrm{P} \alpha-\mathrm{MS}$ & TMPC-P & 180 & $0.21-0.29$ & $0.001-0.006$ & 3 \\
\hline PMMA & $\mathrm{PC}$ & 50 & $0.057-0.066$ & 0.043 & 3 \\
\hline PMMA & DMPC & 196 & 0.16 & - & 3 \\
\hline PMMA & TMPC & 214 & 0.29 & 0.40 & 3 \\
\hline PMMA & TCPC & 300 & $0.45-0.77$ & - & 3 \\
\hline PMMA & PCZ & 50 & 0.17 & - & 3 \\
\hline PMMA & HFPC & 160 & -0.072 & -0.30 & 3 \\
\hline PMMA & $\mathrm{BCPC}$ & 150 & 0.01 & -0.077 & 3 \\
\hline PMMA & TMPC-P & 235 & 0.22 & 0.31 & 3 \\
\hline PS & PSF & 248 & 0.85 & 1.18 & 4 \\
\hline PS & DMPSF & 300 & $>0.67$ & - & 4 \\
\hline PS & TMPSF & 228 & 0.31 & 0.33 & 4 \\
\hline PS & HMBIPSF & 300 & $>1.25$ & - & 4 \\
\hline PS & PES & 300 & -1.27 & - & 4 \\
\hline
\end{tabular}


Table 2.11 (continued)

\begin{tabular}{|c|c|c|c|c|c|c|}
\hline Polymer-1 & Polymer-2 & $T\left({ }^{\circ} \mathrm{C}\right)$ & $B(\mathrm{cal} / \mathrm{mL})$ & $\Delta P^{*}(\mathrm{cal} / \mathrm{mL})$ & $\chi_{12}$ & References \\
\hline PS & HFPSF & 300 & $>136$ & - & & 4 \\
\hline PS & TMHFPSF & 50 & 0.63 & - & & 4 \\
\hline PS & TMHFPSF & 240 & 1.12 & - & & 4 \\
\hline PS & TMPSF-P & 174 & 0.34 & & & 4 \\
\hline $\mathrm{P} \alpha-\mathrm{MS}$ & PSF & 50 & 0.32 & 0.37 & & 4 \\
\hline $\mathrm{P} \alpha-\mathrm{MS}$ & PSF & 300 & $>0.43$ & $>0.53$ & & 4 \\
\hline $\mathrm{P} \alpha-\mathrm{MS}$ & DMPSF & 300 & $>0.30$ & - & & 4 \\
\hline $\mathrm{P} \alpha-\mathrm{MS}$ & TMPSF & 50 & $>0.35$ & $>0.36$ & & 4 \\
\hline $\mathrm{P} \alpha-\mathrm{MS}$ & HMBIPSF & 300 & $>0.29$ & - & & 4 \\
\hline $\mathrm{P} \alpha-\mathrm{MS}$ & PES & 300 & $>0.31$ & - & & 4 \\
\hline $\mathrm{P} \alpha-\mathrm{MS}$ & HFPSF & 50 & $>0.20$ & - & & 4 \\
\hline $\mathrm{P} \alpha-\mathrm{MS}$ & TMHFPSF & 300 & $>0.30$ & - & & 4 \\
\hline $\mathrm{P} \alpha-\mathrm{MS}$ & TMPSF-P & 300 & $>0.29$ & - & & 4 \\
\hline PMMA & PSF & 50 & $0.25-0.34$ & $0.19-0.27$ & & 4 \\
\hline PMMA & DMPSF & 300 & $>0.77$ & - & & 4 \\
\hline PMMA & TMPSF & 231 & 0.39 & 0.44 & & 4 \\
\hline PMMA & HMBIPSF & 300 & $>0.76$ & - & & 4 \\
\hline PMMA & PES & 300 & $>0.78$ & - & & 4 \\
\hline PMMA & HFPSF & 50 & $0.10-0.15$ & - & & 4 \\
\hline PMMA & TMHFPSF & 50 & 0.25 & - & & 4 \\
\hline PMMA & TMHFPSF & 293 & 0.77 & - & & 4 \\
\hline PMMA & TMPSF-P & 300 & $>0.76$ & - & & 4 \\
\hline PAN & PSF & 50 & 3.6 & 4.1 & & 4 \\
\hline PET & PETG & 280 & & & -0.12 & 5 \\
\hline PBT & $20 \% \mathrm{PAr}$ & 249 & & & -0.65 & 6 \\
\hline PBT & $40 \%$ PAr & 246 & & & -0.40 & 6 \\
\hline PBT & $60 \% \mathrm{PAr}$ & 236 & & & -0.31 & 6 \\
\hline PBT & $80 \% \mathrm{PAr}$ & 232 & & & -0.22 & 6 \\
\hline PBT & $\begin{array}{l}65 \mathrm{wt} \% \\
\text { PEE }\end{array}$ & & & & 0.13 & 7 \\
\hline PBT & $\begin{array}{l}70 \mathrm{wt} \% \\
\text { PEE }\end{array}$ & & & & 0.10 & 7 \\
\hline PBT & $\begin{array}{l}75 \mathrm{wt} \% \\
\text { PEE }\end{array}$ & & & & 0.07 & 7 \\
\hline PBT & $\begin{array}{l}80 \mathrm{wt} \% \\
\text { PEE }\end{array}$ & & & & 0.048 & 7 \\
\hline PBT & $\begin{array}{l}85 \mathrm{wt} \% \\
\text { PEE }\end{array}$ & & & & 0.032 & 7 \\
\hline $\mathrm{PBT}$ & $\begin{array}{l}90 \mathrm{wt} \% \\
\text { PEE }\end{array}$ & & & & 0.012 & 7 \\
\hline PS & $\begin{array}{l}75 \% \mathrm{P}_{p^{-}} \\
\mathrm{MS}\end{array}$ & 140 & & & -0.008 & 8 \\
\hline
\end{tabular}


Table 2.11 (continued)

\begin{tabular}{|c|c|c|c|c|c|c|}
\hline Polymer-1 & Polymer-2 & $T\left({ }^{\circ} \mathrm{C}\right)$ & $B(\mathrm{cal} / \mathrm{mL})$ & $\Delta P^{*}(\mathrm{cal} / \mathrm{mL})$ & $\chi_{12}$ & References \\
\hline PS & $\begin{array}{l}50 \% \mathrm{P}_{p^{-}} \\
\mathrm{MS}\end{array}$ & 140 & & & -0.006 & 8 \\
\hline PS & $\begin{array}{l}25 \% \mathrm{P}_{p^{-}} \\
\mathrm{MS}\end{array}$ & 140 & & & -0.006 & 8 \\
\hline $\mathrm{PB}$ & $50 \% d-\mathrm{PB}$ & 130 & & & 0.00053 & 9 \\
\hline $\mathrm{PB}$ & $50 \% d-\mathrm{PB}$ & 121 & & & 0.00055 & 9 \\
\hline $\mathrm{PB}$ & $43 \% d-\mathrm{PB}$ & 130 & & & 0.00032 & 9 \\
\hline PMMA & $\begin{array}{l}50 \% \\
\text { PnBMA }\end{array}$ & 25 & & & 0.081 & 10 \\
\hline PMMA & $\begin{array}{l}50 \% \\
\text { PiBMA }\end{array}$ & 25 & & & 0.068 & 10 \\
\hline PiBMA & $\begin{array}{l}50 \% \\
\text { PnBMA }\end{array}$ & 25 & & & 0.0019 & 10 \\
\hline PS & PCSt & 30 & & & $0.07 \pm 0.02$ & 11 \\
\hline PVDF & PMMA & 170 & -2.93 & & & 12 \\
\hline PVDF & $\begin{array}{l}\text { MMA- } \\
\text { GMA }_{(8)}\end{array}$ & 170 & -4.1 & & & 12 \\
\hline PVDF & $\begin{array}{l}\text { MMA- } \\
\text { GMA }_{(14)}\end{array}$ & 170 & -4.5 & & & 12 \\
\hline PVDF & $\begin{array}{l}\text { MMA- } \\
\text { GMA }_{(28)}\end{array}$ & 170 & -3.7 & & & 12 \\
\hline $\mathrm{PC}$ & PNP & 200 & & & 0.175 & 13 \\
\hline $\mathrm{PC}$ & PMS & 200 & & & 0.031 & 13 \\
\hline PCEMA & PCL & & & & -0.99 & 14 \\
\hline PCEMA & PHS & & & & -0.48 & 14 \\
\hline PS & PAN & & 4.59 & & & 15 \\
\hline $\mathrm{P} \alpha-\mathrm{MS}$ & PAN & & 6.02 & & & 15 \\
\hline $\mathrm{P} \alpha-\mathrm{MS}$ & PS & & $0.022 \pm 0.001$ & & & 16 \\
\hline $\mathrm{BR}$ & PAN & & 8.60 & & & 17 \\
\hline $\mathrm{BR}$ & PVC & & $0.72 \pm 0.07$ & & & 17 \\
\hline PVC & PAN & & $3.84 \pm 0.43$ & & & 17 \\
\hline PVAl & PAA & & -19.9 & & -1.24 & 18 \\
\hline PA-6 & Zn-SPS & 220 & & & -1.3 & 19 \\
\hline PA-6 & Li-SPS & 240 & -215 & & & 20 \\
\hline PA-6 & PS & 240 & 28.7 & & & 20 \\
\hline PVDF & PMMA & 160 & -4.43 & & & 21 \\
\hline PVDF & PEMA & 160 & -2.66 & & & 21 \\
\hline PEMA & PMMA & 160 & 3.25 & & & 21 \\
\hline PA-6 & $\operatorname{Mn}^{-S P S}(10)$ & 190 & -1.9 & & & 22 \\
\hline PA-6 & $\operatorname{Mn}-\mathrm{SPS}_{(20)}$ & 180 & -2.0 & & & 22 \\
\hline PA-6 & Mn-SPS $(30)$ & 175 & -1.8 & & & 22 \\
\hline PA-6 & $\mathrm{Mn} \mathrm{SPS}_{(50)}$ & 166 & -1.5 & & & 22 \\
\hline
\end{tabular}


Table 2.11 (continued)

\begin{tabular}{|c|c|c|c|c|c|c|}
\hline Polymer-1 & Polymer-2 & $T\left({ }^{\circ} \mathrm{C}\right)$ & $B(\mathrm{cal} / \mathrm{mL})$ & $\Delta P^{*}(\mathrm{cal} / \mathrm{mL})$ & $\chi_{12}$ & References \\
\hline PS & PPE & 150 & & & -0.2 & 23 \\
\hline PVC & $\mathrm{PCL}_{(50)}$ & 250 & & & -0.5 & 23 \\
\hline PI & $d-\mathrm{PB}_{(50)}$ & 150 & & & 0.0023 & 24 \\
\hline PHS & PCEMA & & -4.67 & & -0.76 & 25 \\
\hline PHS & PCMMA & & -8.39 & & -1.2 & 25 \\
\hline PHS & PVC & & -3.8 & & -0.76 & 26 \\
\hline PCL & $\operatorname{SAN}_{(25)}$ & & -0.61 & & & 27 \\
\hline PCL & $\mathrm{PC}$ & & -0.39 & & & 27 \\
\hline $\mathrm{PC}$ & $\operatorname{SAN}_{(25)}$ & & $0.2 \pm 0.3$ & & & 27 \\
\hline PVDC & PDPS & & -0.2 & & Fig. 2.7 & 28 \\
\hline PVDC & PDPA & & -1.1 & & Fig. 2.7 & 28 \\
\hline PVDC & PCL & & -2.0 & & Fig. 2.7 & 28 \\
\hline PVDC & PCDS & & -3.1 & & Fig. 2.7 & 28 \\
\hline PVDF & PBA & 175 & -1.0 & & -0.19 & 29 \\
\hline PVME & $d-\mathrm{PS}_{(50)}$ & $100-150$ & & & $0.0702-30.9 / \mathrm{T}$ & 30 \\
\hline PVME & $d-\mathrm{PS}_{(70.6)}$ & $100-150$ & & & $0.0817-36.8 / \mathrm{T}$ & 30 \\
\hline $\mathrm{PB}$ & $d$-PB & $-50-80$ & & & $0.5-1.29$ & 30 \\
\hline PSi $\alpha M S$ & $\mathrm{PS}_{(50)}$ & $100-200$ & & & $0.0032-5.46 / \mathrm{T}$ & 31 \\
\hline $\mathrm{P} \alpha-\mathrm{MS}$ & $\mathrm{PS}_{(50)}$ & $180-300$ & & & $0.0044-0.0046$ & 31 \\
\hline PMMA & PS & & & & $0.006-0.022$ & 32 \\
\hline $\mathrm{P} 4 \mathrm{VP}$ & PS & 165 & & & $7.5 \pm 2.5$ & 33 \\
\hline $\mathrm{P} 4 \mathrm{VP}$ & PS & 180 & & & $3.5 \pm 1.5$ & 33 \\
\hline $\mathrm{P} 4 \mathrm{VP}$ & PS & 183 & & & 0.4 & 33 \\
\hline $\mathrm{PIB}_{[82 k]}$ & $\mathrm{EB}_{[85 k]}$ & & & Fig. 2.8 & $0.0194-6.36 / \mathrm{T}$ & 34 \\
\hline $\mathrm{PIB}_{[82 k]}$ & $\mathrm{EB}_{[114 k]}$ & & & Fig. 2.8 & $\begin{array}{l}0.0232-8.306 / \\
\mathrm{T}\end{array}$ & 34 \\
\hline $\mathrm{PIB}_{[160 k]}$ & $\mathrm{EB}_{[114 k]}$ & & & Fig. 2.8 & $0.0228-8.14 / \mathrm{T}$ & 34 \\
\hline $\mathrm{PIB}_{[82 k]}$ & $\mathrm{EB}_{[73 k]}$ & & & Fig. 2.8 & $\begin{array}{l}0.0151-5.149 / \\
\mathrm{T}\end{array}$ & 34 \\
\hline $\mathrm{PIB}_{[82 k]}$ & $\operatorname{HHPP}_{[27.5 k]}$ & & & Fig. 2.8 & $0.0194-6.36 / \mathrm{T}$ & 34 \\
\hline$d$-PP & HHPP & & & Fig. 2.9 & $\begin{array}{l}-0.00639+ \\
3.305 / \mathrm{T}\end{array}$ & 35 \\
\hline$d$-PP & $\mathrm{EB}_{(97)}$ & & & Fig. 2.9 & $\begin{array}{l}-0.00883+ \\
4.200 / \mathrm{T}\end{array}$ & 35 \\
\hline$d$-PP & $\mathrm{EB}_{(78)}$ & & & Fig. 2.9 & $\begin{array}{l}-0.00320+ \\
1.685 / \mathrm{T}\end{array}$ & 35 \\
\hline$d$-HНPP & PEB & & & Fig. 2.9 & $\begin{array}{l}-0.00137+ \\
1.011 / \mathrm{T}\end{array}$ & 35 \\
\hline$d$-HНPР & PEP & & & Fig. 2.9 & $\begin{array}{l}-0.00036+ \\
0.517 / \mathrm{T}\end{array}$ & 35 \\
\hline PVDF & PMA & 160 & & & $\begin{array}{l}-0.221 \pm \\
0.002\end{array}$ & 36 \\
\hline
\end{tabular}


Table 2.11 (continued)

\begin{tabular}{|c|c|c|c|c|c|c|}
\hline Polymer-1 & Polymer-2 & $T\left({ }^{\circ} \mathrm{C}\right)$ & $B(\mathrm{cal} / \mathrm{mL})$ & $\Delta P^{*}(\mathrm{cal} / \mathrm{mL})$ & $\chi_{12}$ & References \\
\hline $\begin{array}{l}\mathrm{P}\left(\mathrm{VF}_{2^{-}}\right. \\
\left.\text {co- }-\mathrm{VF}_{4}\right)\end{array}$ & PMA & 160 & & & $\begin{array}{l}-0.005 \pm \\
0.0005\end{array}$ & 36 \\
\hline HDPE & LDPE & 150 & & & $\begin{array}{l}0.000402 \pm \\
4 \times 10^{-5}\end{array}$ & 37 \\
\hline HDPE & LDPE & 180 & & & $\begin{array}{l}0.000390 \pm \\
4 \times 10^{-5}\end{array}$ & 37 \\
\hline HDPE & LDPE & 190 & & & $\begin{array}{l}0.000387 \pm \\
4 \times 10^{-5}\end{array}$ & 37 \\
\hline $\mathrm{PEP}_{(25)}$ & PEB & $27-167$ & & & $\begin{array}{l}-0.00167+ \\
0.954 / T\end{array}$ & 38 \\
\hline $\operatorname{PEP}_{(57.5)}$ & PEB & $27-167$ & & & $\begin{array}{l}-0.00143+ \\
0.883 / \mathrm{T}\end{array}$ & 38 \\
\hline $\mathrm{PEP}_{(89.1)}$ & PEB & $27-167$ & & & $\begin{array}{l}-0.00219+ \\
1.138 / \mathrm{T}\end{array}$ & 38 \\
\hline $\operatorname{PEMA}_{(70)}$ & $\mathrm{CR}$ & 42 & & & -0.122 & 39 \\
\hline $\mathrm{PEMA}_{(50)}$ & $\mathrm{CR}$ & 40 & & & -0.053 & 39 \\
\hline $\operatorname{PEMA}_{(30)}$ & $\mathrm{CR}$ & 39 & & & -0.030 & 39 \\
\hline PCL & P4HS & 50 & & & -1.1 & 40 \\
\hline PVP & $\begin{array}{l}\text { CDA } 90 \mathrm{wt} \\
\%\end{array}$ & 24.5 & & & -4.20 & 41 \\
\hline PVP & $\begin{array}{l}\text { CDA } 65 \mathrm{wt} \\
\%\end{array}$ & 24.5 & & & -1.64 & 41 \\
\hline PVP & $\begin{array}{l}\text { CDA } 40 \mathrm{wt} \\
\%\end{array}$ & 24.5 & & & -0.60 & 41 \\
\hline PVP & $\begin{array}{l}\text { CDA } 15 \mathrm{wt} \\
\%\end{array}$ & 24.5 & & & -0.36 & 41 \\
\hline PMMA & Phenoxy & 170 & & & -0.61 & 42 \\
\hline PMMA & PEG & & & & -0.35 & 43 \\
\hline PEG & Phenoxy & & & & -1.90 & 44 \\
\hline PA-6 & MXD & 275 & & & $\begin{array}{l}-0.185 / \\
-0.194\end{array}$ & 45 \\
\hline PEEK & PEI & 180 & & & -0.3 & 46 \\
\hline $\mathrm{PP}$ & SEBS + oil & 160 & & & -0.043 & 47 \\
\hline $\left.\mathrm{PCl}_{(\text {high } \phi}\right)$ & PVDC & & & & -0.02 & 48 \\
\hline $\left.\mathrm{PCl}_{(\text {low } \phi}\right)$ & PVDC & & & & -0.21 & 48 \\
\hline $\left.\mathrm{PCl}_{\text {(high } \phi)}\right)$ & $\begin{array}{l}\mathrm{P}\left(\mathrm{VCl}_{2^{-}}\right. \\
\mathrm{VAc})\end{array}$ & & & & -0.01 & 48 \\
\hline $\mathrm{PCl}_{(\text {low } \phi)}$ & $\begin{array}{l}\mathrm{P}\left(\mathrm{VCl}_{2^{-}}\right. \\
\mathrm{VAc})\end{array}$ & & & & -0.28 & 48 \\
\hline PS & PPE & 210 & $-0.89 \pm 0.04$ & & & 49 \\
\hline PS & PPE & 210 & $-0.31 \pm 0.15$ & & & 50 \\
\hline PS & PPE & 232 & $-1.62 \pm 0.07$ & & & 51 \\
\hline PS & PPE & 210 & $\begin{array}{l}-1.46+ \\
0.00238 \mathrm{~T}\end{array}$ & & $0.121-77.9 / \mathrm{T}$ & 52 \\
\hline
\end{tabular}


Table 2.11 (continued)

\begin{tabular}{lllllll}
\hline Polymer-1 & Polymer- & $T\left({ }^{\circ} \mathrm{C}\right)$ & $B(\mathrm{cal} / \mathrm{mL})$ & $\Delta P^{*}(\mathrm{cal} / \mathrm{mL})$ & $\chi_{12}$ & References \\
\hline PS & P1,4PE & 30 & $-0.005 \pm 0.43$ & & 53 \\
PS & PC & 30 & $0.41 \pm 0.13$ & & 54 \\
PS & TMPC & 30 & $0.19 \pm 0.34$ & & 54 \\
PS & PAN & 30 & $7.63 \pm 0.12$ & & 54 \\
\hline PES-C & PEG & 65 & 4.65 & & 55 \\
\hline
\end{tabular}

Abbreviations: $B C P C$ bisphenol chloral polycarbonate, $C D A$ cellulose diacetate, $C R$ polychloroprene, $D M P C$ dimethyl bisphenol-A polycarbonate, DMPSF dimethyl bisphenol-A polysulfone, $E B$ ethylene-butene copolymer (with indicated, in subscripts, butene content, or MW in $\mathrm{g} / \mathrm{mol}$ ), HFPC hexafluoro bisphenol-A polycarbonate, HFPSF hexafluoro polysulfone, $H M B I P S F$ hexamethyl biphenol polysulfone, $M X D$ poly $(m$-xylene adipamide), $P 1,4 P E$ poly (1,4-phenylene ether), $P 4 V P$ poly(4-vinylpyridine), $P A N$ polyacrylonitrile, $P B A$ poly (1,4-butylene adipate), $P B$ polybutadiene, $d-P B$ deuterated polybutadiene; $P C$ bisphenol-A polycarbonate, $P C D S$ poly(1,4-cyclohexanedimethylene succinate), $P C E M A$ poly-1-chloroethyl methacrylate, $P C M M A$ poly-1-chloromethyl methacrylate, $P C Z$ bisphenol-Z polycarbonate, $P D P A$ poly(2,2-dimethyl-1,3-propylene adipate), $P D P S$ poly(2,2-dimethyl-1,3-propylene succinate), $P E B$ polyethylene-butene, $P E E$ polyester-ether aromatic block copolymer, $P E P$ polyethylenepropylene, $P E S$ - $C$ phenolphthalein polyetherether sulfone, $P E S$ polyethersulfone, $P H S$ polyhexamethylene sebacate, $P I$ polyisoprene, $P M S$ poly(methylmethacrylate-co- $p$-methylstyrene), $P N P$ poly(methylmethacrylate- $c o-N$-phenylmaleimide), $d-P P$ deuterated amorphous polypropylene, HHPP head-to-head amorphous polypropylene, $d$-HHPP deuterated head-to-head amorphous polypropylene, $P P E$ poly(2,6-dimethyl-1,4-phenylene ether), $P S F$ bisphenol-A polysulfone, $P 4 H S$ poly(4-hydroxy styrene), $P S$ polystyrene, $P C S t$ poly-p-chlorostyrene, $P \alpha-M S$ poly( $\alpha$-methylstyrene), $P S i \alpha M S$ poly(sila- $\alpha$-methylstyrene), $P p-M S$ poly-para-methylstyrene, $S A N_{(x)}$ copolymer of styrene with $x \%$ acrylonitrile, Li-SPS Li-sulfonated PS, Mn-SPS Mn-sulfonated PS, Zn-SPS Zn-sulfonated PS, TCPC tetrachloro bisphenol-A polycarbonate, $T M P C$ - $P$ tetramethyl bisphenol-P polycarbonate, $T M P C$ tetramethyl bisphenol-A polycarbonate, TMHFPSF tetramethyl hexafluoro polysulfone, TMPSF-P tetramethyl bisphenol-P polysulfone, TMPSF tetramethyl bisphenol-A polysulfone

References: 1. Kim and Paul 1992; 2. Callaghan and Paul 1993; 3. Callaghan and Paul 1994a; 4. Callaghan and Paul 1994b; 5. Papadopoulou and Kalfoglu 1997; 6. Huo and Cebe 1993; 7. Gallagher et al. 1993; 8. Londono and Wignall 1997; 9. Alamo et al. 1997; 10. Sato et al. 1996a, b; 11. Ogawa et al. 1986; 12. Gan and Paul 1995; 13. Ikawa and Hosoda 1991; 14. Peng et al. 1994; 15. Cowie et al. 1992a; 16. Cowie et al. 1992b; 17. Cowie and Harris 1992; 18. Dinililuc et al. 1992; 19. Lu and Weiss 1992; 20. Molnar and Eisenberg 1992; 21. Goh and Siow 1988; 22. Lu and Weiss 1991; 23. Lu and Weiss 1992; 24. Hasegawa et al. 1991; 25. Neo and Goh 1992; 26. Woo et al. 1985; 27. Shah et al. 1986; 28. Woo et al. 1983; 29. Pennings and Manley 1996; 30. Takeno et al. 1996; 31. Maier et al. 1996; 32. Pinder 1997; 33. Clarke et al. 1997; 34. Krishnamoorti et al.; 35. Graessley et al. 1995; 36. Maiti and Nandi 1995; 37. Schipp et al. 1996; 38. Lin et al. 1995; 39. Kundu et al. 1996; 40. Lezcano et al. 1996; 41. Jinghua et al. 1997; 42. Hong et al. 1997; 43. Martuscelli et al. 1984; 44. Iriarte et al. 1989; 45. Shibayama et al. 1995; 46. Goodwin and Simon 1996; 47. Ohlsson and Tørnell 1996; 48. Aubin et al. 1983; 49. ten Brinke et al. 1983; 50. Kambour et al. 1980; 51. Plans et al. 1984; 52. Maconnachie et al. 1984; 53. Ziaee and Paul 1996; 54. Ziaee and Paul 1997; 55. Zheng et al. 1997

Determination of $B$ for a series of blends made it possible to establish empirical rules for the observed miscibilities. Thus, for example, Prud'homme (1982) reported a systematic variation of miscibility in a series of halogenated polymer blends with aliphatic polyesters. The highest miscibility was observed for 
Fig. 2.9 The binary interaction parameter $B$ for PVDC/aliphatic polyester blends plotted as a function of the number of methylene groups $\left(-\mathrm{CH}_{2}-\right)$ per ester group (-COO-) in the second polymer (Data from Woo et al. 1986)
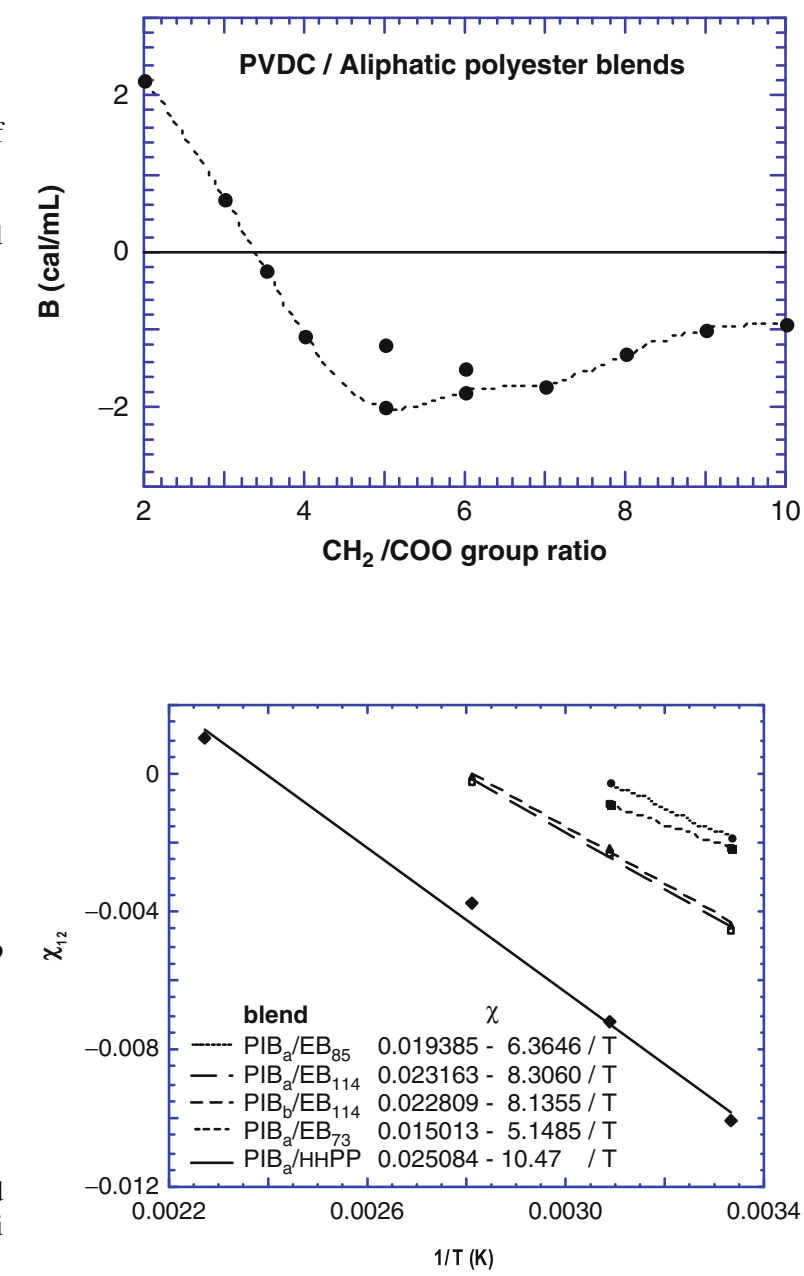

Fig. 2.10 The binary interaction parameters for two PIB resins $\left(M_{w}=81.6\right.$ and $114 \mathrm{~kg} / \mathrm{mol}$ ) with either ethylene-butene copolymers (of different $M_{w}$ and composition) or with an atactic head-to-head polypropylene (HHPP), based on SANS data (Krishnamoorti et al. 1995)

10

.


Fig. 2.11 Temperature dependence of the binary interaction parameter, $\chi$, for blends of deuterated amorphous polypropylene (either head-to-tail or head-tohead) with polyethylene copolymers. The values were determined from SANS data (Graessley et al. 1995)
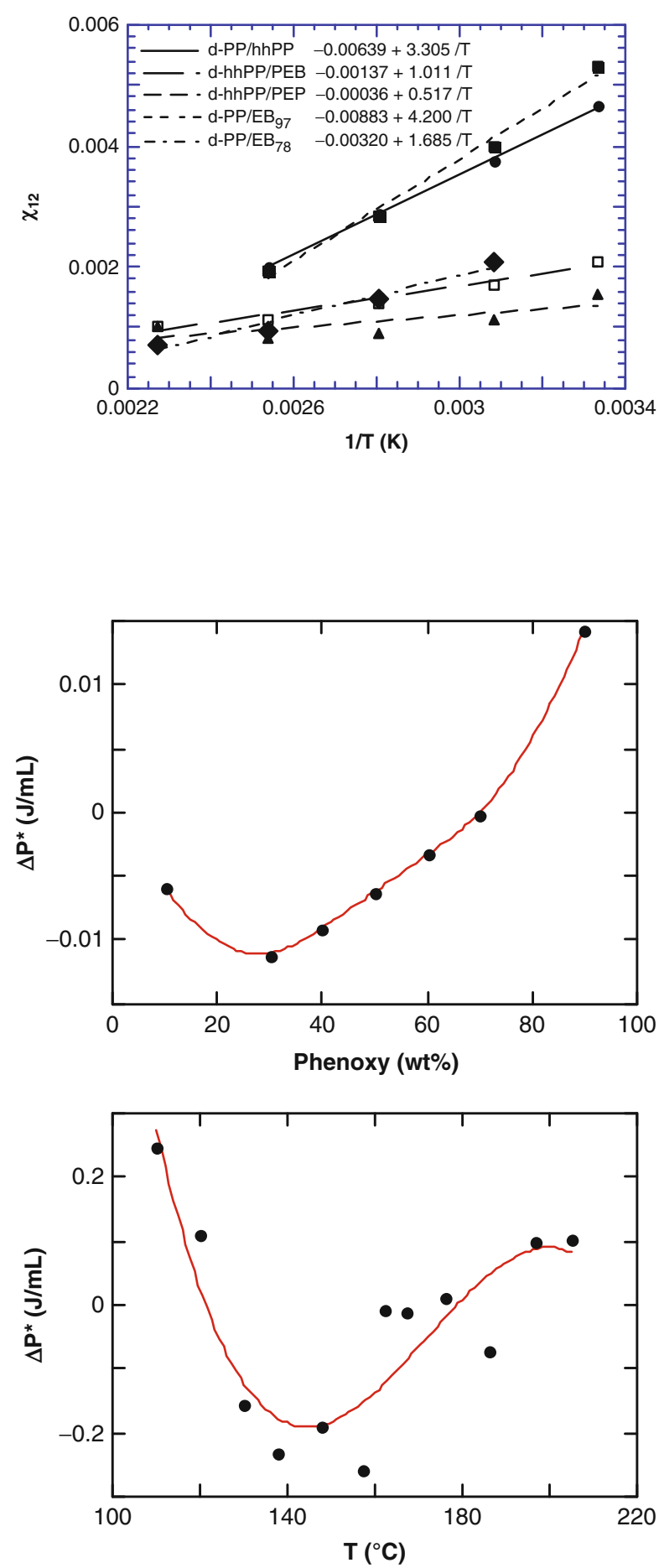

Fig. 2.12 (Top)

Compositional variation of the interaction parameter $\Delta P^{*}$ in phenoxy/polyvinyl methyl ether blends at the spinodal temperature, $T_{s}$. (bottom) Temperature dependence of $\Delta P *$ (Data after Etxeberria et al. 1997)$$
0
$$ 
Fig. 2.13 Compositional variation of the interaction parameter, $B$, in a copolymer (mers \#1 and \#2)/

homopolymer (mers \#3) blend. Even when all three binary interaction parameters are positive

$\left(B_{23}=1, B_{13}=2\right.$, and $B_{12}=0-10$ ), for a copolymer with strong repulsive interactions, $B_{12}=10$, a "window of miscibility" is predicted
Copolymer-A (mers \#1 \& \#2) with polymer-B (mers \#3) blends:

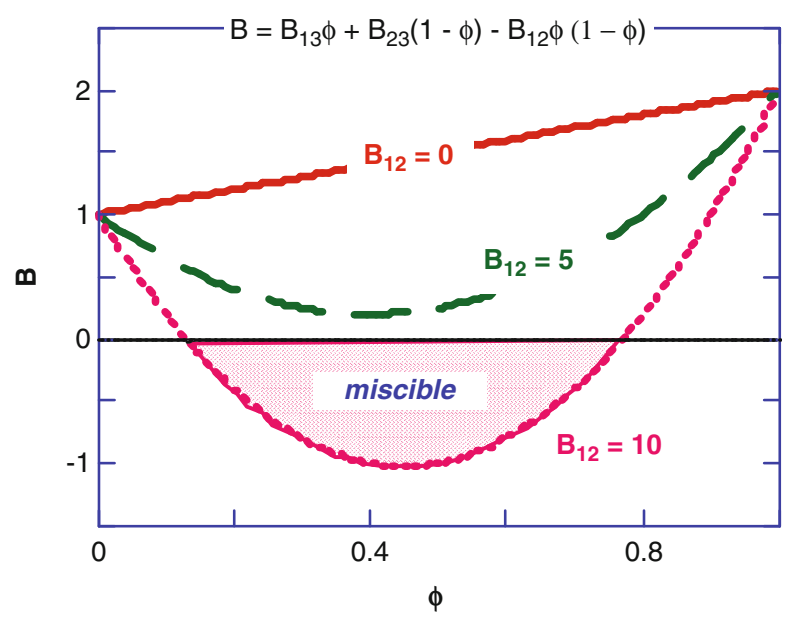

The observed regularity in the miscibility behavior of polymers can be understood considering the polymers as composed of individual interacting groups. For example, taking the case illustrated in Fig. 2.9, one may consider that PVDC is an alternating copolymer of units, $-\mathrm{CH}_{2}-$ and $-\mathrm{CCl}_{2}-$, whereas the aliphatic polyester is composed of $-\mathrm{CH}_{2}-$ and $-\mathrm{COO}-$ units. Equations 2.48 and 2.49 predict that even systems with all positive values of the binary interaction parameter, $B_{i j}>0$, (repulsive interactions) may have a window of miscibility, where the overall parameter $B$ becomes negative. The magnitude of this effect depends primarily on the value of the repulsive interactions within the copolymer molecule, $B_{12}>0$; a schematic representation of Eq. 2.49 is shown in Fig. 2.13.

Since $B_{i j}$ characterizes the enthalpic and non-configurational entropy of interaction between segments $i$ and $j$, they should be pairwise and additive, to a good approximation; thus, one could tabulate these parameters per group and, in turn, use them for predicting miscibility in any arbitrary system composed of polymers and/or copolymers built from these groups/segments (Paul and Barlow 1984). This idea is similar in concept with the group-contribution approach of calculating solubility parameters. Table 2.12 provides examples of $B_{i j}$ values published over the years, as well as $\chi_{i j}$. The $\chi_{i j}$ dimensionless parameter is directly proportional to $B_{i j}$ (Eq. 2.47), whereas $\Delta P^{*}$ is the binary interaction energy density calculated from S-L EoS. Since $\Delta P^{*}$ is also proportional to $B_{i j}$ (Sanchez 1989 ), one may apply S-L theory to experimental data and compute the segmental contributions, $\Delta P_{i j}^{*}$, in analogy to $B_{i j}$ :

$$
\Delta P^{*}=\Delta P_{12}^{*} \phi_{2}^{\prime}\left(\phi_{2}^{\prime}-\phi_{3}^{\prime \prime}\right)+\Delta P_{13}^{*} \phi_{3}^{\prime \prime}\left(\phi_{3}^{\prime \prime}-\phi_{2}^{\prime}\right)+\Delta P_{23}^{*} \phi_{2}^{\prime} \phi_{3}^{\prime \prime}
$$

The segmental interaction parameters have been also used to explain the "miscibility window" or "miscibility chimney" in polymer/copolymer or 
Table 2.12 Binary segmental interaction parameters, $B_{i j}$ or $\chi_{i j}$

\begin{tabular}{|c|c|c|c|c|}
\hline Unit 1 & Unit 2 & $B_{i j}(\mathrm{cal} / \mathrm{mL})$ & $\chi_{i j}$ & Reference \\
\hline$-\mathrm{CH}_{2-}$ & Phenoxy & 8.34 & & 1 \\
\hline$-\mathrm{CH}_{2-}$ & $-\mathrm{COO}-$ & 250.3 & & 1 \\
\hline$-\mathrm{COO}-$ & Phenoxy & 154.9 & & 1 \\
\hline$-\mathrm{CH}_{2}-$ & $\mathrm{S}$ & 5.72 & & 2 \\
\hline$-\mathrm{CH}_{2-}$ & AN & 97.5 & & 2 \\
\hline$-\mathrm{CH}_{2-}$ & $-\mathrm{COO}-$ & 136.5 & & 2 \\
\hline $\mathrm{S}$ & AN & 122 & & 2 \\
\hline$S$ & $-\mathrm{COO}-$ & 103 & & 2 \\
\hline $\mathrm{AN}$ & $-\mathrm{COO}-$ & 104 & & 2 \\
\hline$-\mathrm{CH}_{2-}$ & $-\mathrm{CH}\left(\mathrm{C}_{6} \mathrm{H}_{5}\right)-$ & 8.07 & & 2 \\
\hline$-\mathrm{CH}_{2}-$ & $-\mathrm{CH}(\mathrm{CN})-$ & 234 & & 2 \\
\hline$-\mathrm{CH}\left(\mathrm{C}_{6} \mathrm{H}_{5}\right)-$ & $-\mathrm{CH}(\mathrm{CN})-$ & 277 & & 2 \\
\hline$-\mathrm{CH}\left(\mathrm{C}_{6} \mathrm{H}_{5}\right)-$ & $-\mathrm{COO}-$ & 96.8 & & 2 \\
\hline$-\mathrm{CH}(\mathrm{CN})-$ & $-\mathrm{COO}-$ & 170 & & 2 \\
\hline$-\mathrm{CH}_{x}-$ & $\left(\mathrm{C}_{6} \mathrm{H}_{5}\right)_{-}$ & 10.4 & & 2 \\
\hline$-\mathrm{CH}_{x}-$ & $-\mathrm{CN}$ & 508 & & 2 \\
\hline$-\mathrm{CH}_{x}-$ & $-\mathrm{COO}-$ & 136 & & 2 \\
\hline$\left(\mathrm{C}_{6} \mathrm{H}_{5}\right)_{-}$ & $-\mathrm{CN}$ & 579 & & 2 \\
\hline$\left(\mathrm{C}_{6} \mathrm{H}_{5}\right)_{-}$ & $-\mathrm{COO}-$ & 93.0 & & 2 \\
\hline$-\mathrm{CN}$ & $-\mathrm{COO}-$ & 351 & & 2 \\
\hline VAc & $\mathrm{C}(\mathrm{VC})$ & & 0.17 & 3 \\
\hline VAc & $\mathrm{VC}$ & & 0.27 & 3 \\
\hline VAc & $-\mathrm{CH}_{2-}$ & & 1.01 & 3 \\
\hline $\mathrm{VC}$ & $\mathrm{C}(\mathrm{VC})$ & & 0.042 & 3 \\
\hline $\mathrm{VC}$ & $-\mathrm{CH}_{2-}$ & & 0.15 & 3 \\
\hline $\mathrm{S}$ & MMA & 0.22 & & 4 \\
\hline $\mathrm{S}$ & MA & 7.32 & & 4 \\
\hline MA & MMA & 9.30 & & 5 \\
\hline MA & EMA & 10.4 & & 5 \\
\hline MA & $\mathrm{nPNM}$ & 11.9 & & 5 \\
\hline MA & $S$ & 14.9 & & 5 \\
\hline MA & AN & -0.381 & & 5 \\
\hline $\mathrm{AN}$ & MMA & 5.00 & & 5 \\
\hline $\mathrm{AN}$ & EMA & 5.33 & & 5 \\
\hline $\mathrm{AN}$ & nPMA & 5.85 & & 5 \\
\hline$\overline{\mathrm{AN}}$ & $\mathrm{S}$ & 8.14 & & 5 \\
\hline $\mathrm{S}$ & MMA & 0.181 & & 5 \\
\hline $\mathrm{S}$ & EMA & -0.0361 & & 5 \\
\hline $\mathrm{S}$ & nPMA & -0.0309 & & 5 \\
\hline $\mathrm{S}$ & MMA & 0.26 & & 6 \\
\hline$\alpha-\mathrm{MS}$ & MMA & 0.26 & & 6 \\
\hline$\alpha-\mathrm{MS}$ & $\mathrm{S}$ & 0.018 & & 6 \\
\hline TMPC & $\mathrm{S}$ & -0.15 & & 7 \\
\hline
\end{tabular}


Table 2.12 (continued)

\begin{tabular}{|c|c|c|c|}
\hline Unit 1 & Unit 2 & $B_{i j}(\mathrm{cal} / \mathrm{mL}) \quad \chi_{i j}$ & Reference \\
\hline TMPC & MMA & 0.26 & 7 \\
\hline HFPC & $\mathrm{S}$ & 1.5 & 7 \\
\hline HFPC & MMA & $\begin{array}{l}-0.07 \\
-0.73\end{array}$ & 7 \\
\hline HFPC & TMPC & $0.25 \pm 0.04$ & 7 \\
\hline$S$ & MMA & $0.18,0.26$ & 7 \\
\hline $\mathrm{S}$ & MMA & 0.22 & 8 \\
\hline MA & MMA & 7.18 & 8 \\
\hline $\mathrm{S}$ & MA & 10.7 & 8 \\
\hline$S$ & AN & 6.8 & 8 \\
\hline MA & AN & -0.31 & 8 \\
\hline $\mathrm{S}$ & TMPC & -0.14 & 8 \\
\hline MA & TMPC & 11.5 & 8 \\
\hline $\mathrm{S}$ & PPE & $\leq-0.37$ & 8 \\
\hline MA & PPE & $14.6 \pm 0.5$ & 8 \\
\hline MMA & $\mathrm{VC}$ & 0.54 & 9 \\
\hline $\mathrm{S}$ & $\mathrm{VC}$ & 2.85 & 9 \\
\hline MMA & $\mathrm{S}$ & 2.93 & 9 \\
\hline $\mathrm{AN}$ & $\mathrm{S}$ & 95.52 & 9 \\
\hline TMPAr & PAr & 5.36 & 10 \\
\hline TMPAr & $\mathrm{S}$ & 2.18 & 10 \\
\hline TMPAr & AN & 99.04 & 10 \\
\hline $\mathrm{PAr}$ & $\mathrm{S}$ & 10.52 & 10 \\
\hline $\mathrm{PAr}$ & AN & 89.09 & 10 \\
\hline $\mathrm{S}$ & AN & 117.89 & 10 \\
\hline$-p \mathrm{C}_{6} \mathrm{H}_{4}-\mathrm{O}-$ & $-p \mathrm{C}_{6} \mathrm{H}_{4}-\mathrm{CO}-$ & 1.44 & 11 \\
\hline $\mathrm{t}-\mathrm{BMA}$ & $\mathrm{S}\left(130^{\circ} \mathrm{C}\right)$ & 0.34 & 12 \\
\hline $\mathrm{t}-\mathrm{BMA}$ & $\mathrm{S}\left(150^{\circ} \mathrm{C}\right)$ & 0.50 & 12 \\
\hline $\mathrm{t}-\mathrm{BMA}$ & $\mathrm{S}\left(180^{\circ} \mathrm{C}\right)$ & 3.03 & 12 \\
\hline $\mathrm{t}-\mathrm{BMA}$ & MAN $\left(130^{\circ} \mathrm{C}\right)$ & 1.47 & 12 \\
\hline $\mathrm{t}-\mathrm{BMA}$ & $\operatorname{MAN}\left(150^{\circ} \mathrm{C}\right)$ & 2.29 & 12 \\
\hline $\mathrm{t}-\mathrm{BMA}$ & MAN $\left(180^{\circ} \mathrm{C}\right)$ & 7.40 & 12 \\
\hline $\mathrm{t}-\mathrm{BMA}$ & $\operatorname{MMN}\left(130^{\circ} \mathrm{C}\right)$ & 0.76 & 12 \\
\hline $\mathrm{t}-\mathrm{BMA}$ & $\operatorname{MMN}\left(150^{\circ} \mathrm{C}\right)$ & 1.00 & 12 \\
\hline $\mathrm{t}-\mathrm{BMA}$ & $\operatorname{MMN}\left(180^{\circ} \mathrm{C}\right)$ & 4.37 & 12 \\
\hline $\mathrm{S}$ & MAN $\left(130^{\circ} \mathrm{C}\right)$ & 1.20 & 12 \\
\hline $\mathrm{S}$ & MAN $\left(150^{\circ} \mathrm{C}\right)$ & 2.02 & 12 \\
\hline $\mathrm{S}$ & MAN $\left(180^{\circ} \mathrm{C}\right)$ & 2.15 & 12 \\
\hline $\mathrm{S}$ & $\operatorname{MMA}\left(130^{\circ} \mathrm{C}\right)$ & 0.221 & 12 \\
\hline $\mathrm{S}$ & $\operatorname{MMA}\left(150^{\circ} \mathrm{C}\right)$ & 0.229 & 12 \\
\hline $\mathrm{S}$ & MMA $\left(180^{\circ} \mathrm{C}\right)$ & 0.242 & 12 \\
\hline $\mathrm{S}$ & CHMA $\left(130^{\circ} \mathrm{C}\right)$ & -0.15 & 12 \\
\hline$S$ & CHMA $\left(150^{\circ} \mathrm{C}\right)$ & -0.43 & 12 \\
\hline
\end{tabular}


Table 2.12 (continued)

\begin{tabular}{|c|c|c|c|c|}
\hline Unit 1 & Unit 2 & $B_{i j}(\mathrm{cal} / \mathrm{mL})$ & $\chi_{i j}$ & Reference \\
\hline $\mathrm{S}$ & $\mathrm{CHMA}\left(180^{\circ} \mathrm{C}\right)$ & -0.10 & & 12 \\
\hline MMA & $\operatorname{MAN}\left(130^{\circ} \mathrm{C}\right)$ & 0.28 & & 12 \\
\hline MMA & MAN $\left(150^{\circ} \mathrm{C}\right)$ & 0.84 & & 12 \\
\hline MMA & $\operatorname{MAN}\left(180^{\circ} \mathrm{C}\right)$ & 0.91 & & 12 \\
\hline CHMA & $\operatorname{MAN}\left(130^{\circ} \mathrm{C}\right)$ & 1.40 & & 12 \\
\hline CHMA & $\operatorname{MAN}\left(150^{\circ} \mathrm{C}\right)$ & 2.65 & & 12 \\
\hline CHMA & $\operatorname{MAN}\left(180^{\circ} \mathrm{C}\right)$ & 2.11 & & 12 \\
\hline CHMA & $\operatorname{MMA}\left(130^{\circ} \mathrm{C}\right)$ & 0.75 & & 12 \\
\hline CHMA & $\operatorname{MMA}\left(150^{\circ} \mathrm{C}\right)$ & 0.64 & & 12 \\
\hline CHMA & MMA $\left(180^{\circ} \mathrm{C}\right)$ & 0.50 & & 12 \\
\hline $\mathrm{S}\left(140-170^{\circ} \mathrm{C}\right)$ & MMA & & $-0.635+287 / \mathrm{T}$ & 13 \\
\hline $\mathrm{S}\left(140-170^{\circ} \mathrm{C}\right)$ & $\mathrm{AN}$ & & $-11.0+4,940 / \mathrm{T}$ & 13 \\
\hline $\mathrm{S}\left(140-170^{\circ} \mathrm{C}\right)$ & $\mathrm{CL}$ & & $-0.913+412 / \mathrm{T}$ & 13 \\
\hline $\mathrm{AN}\left(140-170^{\circ} \mathrm{C}\right)$ & MMA & & $-4.44+2,000 / \mathrm{T}$ & 13 \\
\hline $\mathrm{AN}\left(140-170^{\circ} \mathrm{C}\right)$ & $\mathrm{CL}$ & & $-4.76+2,140 / \mathrm{T}$ & 13 \\
\hline $\begin{array}{l}\text { MMA } \\
\left(130-200^{\circ} \mathrm{C}\right)\end{array}$ & nBMA & & $-0.216+95.7 / \mathrm{T}$ & 14 \\
\hline $\begin{array}{l}\text { MMA } \\
\left(130-200^{\circ} \mathrm{C}\right)\end{array}$ & iBMA & & $-0.157+73.1 / \mathrm{T}$ & 14 \\
\hline $\begin{array}{l}\text { iBMA } \\
\left(130-200^{\circ} \mathrm{C}\right)\end{array}$ & nBMA & & $0.0179-10-7(846 \mathrm{~T}-\mathrm{T} 2)$ & 14 \\
\hline $\mathrm{S}\left(80-130^{\circ} \mathrm{C}\right)$ & $\mathrm{AN}$ & & 0.12 & 15 \\
\hline $\mathrm{S}\left(80-130^{\circ} \mathrm{C}\right)$ & $\mathrm{CL}$ & & 0.0077 & 15 \\
\hline $\mathrm{CL}\left(80-130^{\circ} \mathrm{C}\right)$ & $\mathrm{AN}$ & & 0.049 & 15 \\
\hline $\mathrm{CL}$ & DTC & & 0.001 & 16 \\
\hline $\mathrm{S}$ & DTC & & 0.02 & 16 \\
\hline $\mathrm{AN}$ & DTC & & 0.042 & 16 \\
\hline VCVAc-90 & iBMA & -5.49 & & 17 \\
\hline VCVAc-90 & iBMA-nBMA50 & -2.22 & & 17 \\
\hline VCVAc-90 & iBMA-nBMA13 & -0.11 & & 17 \\
\hline VCVAc-90 & nBMA & -1.73 & & 17 \\
\hline iBMA & nBMA & 0.00002 & & 17 \\
\hline $\mathrm{SAN}_{(75 \% \mathrm{~S})}$ & $\begin{array}{l}\operatorname{SMMA}_{(90 \%} \% \\
\text { MMA) }\end{array}$ & & -0.0134 & 18 \\
\hline $\operatorname{MAN}_{(90 \%} \%$ MMA $)$ & $\begin{array}{l}\operatorname{SMMA}_{(90 \%} \% \\
\text { MMA) }\end{array}$ & & 0.0083 & 18 \\
\hline $\mathrm{MAN}_{(90 \%} \%$ MMA) & $\mathrm{SAN}_{(75 \% \mathrm{~S})}$ & & -0.0108 & 18 \\
\hline $\mathrm{SAN}_{(80 \% \mathrm{~S})}$ & $\begin{array}{l}\operatorname{SMMA}_{(95} \% \\
\text { MMA) }\end{array}$ & & -0.0154 & 18 \\
\hline $\operatorname{MAN}_{(95} \%$ MMA) & $\begin{array}{l}\operatorname{SMMA}_{(95} \% \\
\text { MMA) }\end{array}$ & & 0.0021 & 18 \\
\hline $\operatorname{MAN}_{(95} \%$ MMA) & $\mathrm{SAN}_{(80 \% \mathrm{~S})}$ & & -0.011 & 18 \\
\hline$-\mathrm{CH}_{2}-$ & -NHCO- & & 8.534 & 19 \\
\hline$-\mathrm{CH}_{2}-$ & $-\mathrm{COO}-$ & & 2.233 & 19 \\
\hline
\end{tabular}


Table 2.12 (continued)

\begin{tabular}{lllll}
\hline Unit 1 & Unit 2 & $B_{i j}(\mathrm{cal} / \mathrm{mL})$ & $\chi_{i j}$ & Reference \\
\hline$-\mathrm{CH}_{2}-$ & $-\mathrm{CHCl}-$ & & 0.500 & 19 \\
\hline$-\mathrm{COO}-$ & $-\mathrm{NHCO}-$ & & 3.880 & 19 \\
\hline$-\mathrm{CHCl}-$ & $-\mathrm{NHCO}-$ & & 6.750 & 19 \\
\hline$-\mathrm{COO}-$ & $-\mathrm{CHCl}-$ & & 0.038 & 19 \\
\hline $\mathrm{S}$ & VME & 0.0167 & & 20 \\
$\mathrm{~S}$ & DNS & 1.79 & & 20 \\
\hline DNS & VME & 1.50 & & 20 \\
DNS & PPE & 2.936 & & 20 \\
\hline
\end{tabular}

Abbreviations for the polymeric units: $\left(\mathrm{C}_{6} \mathrm{H}_{5}\right)-$ phenyl ring, $\alpha-M S$ alpha-methylstyrene, $A N$ acrylonitrile, BMA butylmethacrylate, CHMA cyclohexyl methacrylate, $C L$ caprolactone, $C(V C)$ unit of chlorinated PVC, DNS 2,4-dinitrostyrene-co-styrene, DTC 2,2-dimethyltrimethylenecarbonate, $H F P C$ hexafluoro bisphenol-A carbonate, $M A$ maleic anhydride, $M M A$ methylmethacrylate, $P A r$ unit of polyarylate, Phenoxy unit of poly(hydroxy ether) of bisphenol-A, $P P E$ unit of poly(2,6-dimethyl-1,4-phenylene ether), $S$ styrene, TMPAr unit of tetramethyl bisphenol-A polyarylate, TMPC unit of tetramethyl bisphenol-A polycarbonate, $V A c$ vinyl acetate, $V C$ vinyl chloride, $V C V A c 90$ VC-co-VAc copolymer with $90 \%$ VC, $V M E$ vinyl methyl ether

References: 1. Paul and Barlow 1984; 2. Fernandes et al. 1986; 3. Shiomi et al. 1986; 4. Kim et al. 1989; 5. Brannock et al. 1991; 6. Callaghan and Paul 1993; 7. Takakuwa et al. 1994; 8. Gan and Paul 1994a; 9. Dompas et al. 1997; 10. Ahn et al. 1997a, b; 11. Harris and Robeson 1987; 12. Nishimoto et al. 1995; 13. Higashida et al. 1995; 14. Sato et al. 1996a, b; 15. Schulze et al. 1993; 16. Kammer and Kumerlowe 1996; 17. Sato et al. 1997; 18. Cowie et al. 1992c; 19. van Ekenstein et al. 1997; 20. Fernandez et al. 1997

copolymer/copolymer blends (Lath and Cowie 1988). These parameters have been found useful to predict miscibility of blends containing one component whose structure is systematically varied, e.g., polyesters with either halogenated polymers or phenoxy (Prud'homme 1982; Harris et al. 1983; Woo et al. 1985, 1986), polyamide blends (Ellis 1989), ternary blends (Shah et al. 1986), and other systems, viz., SAN/PMMA, SAN/PC, polyethyloxazoline/polyester, PPE with a mixture of $\mathrm{PoClS}$ and $\mathrm{P} p \mathrm{ClS}, \mathrm{PC} / \mathrm{PCL} /$ phenoxy, and many more.

Ellis $(1988,1989,1990 \mathrm{a}, \mathrm{b})$ used the same approach to evaluate miscibility of polyamide blends. He treated the polyamide molecules as copolymers, viz., comprised of units $\mathrm{A}, \mathrm{B}$, and $\mathrm{C}$ in the form $\mathrm{A}_{\mathrm{x}} \mathrm{B}_{\mathrm{y}} \mathrm{C}_{1-\mathrm{x}-\mathrm{y}}$, where $\mathrm{A},-\mathrm{CH}_{2}-; \mathrm{B},-\mathrm{NHCO}-$; and $\mathrm{C},-\mathrm{C}_{6} \mathrm{H}_{4}-$ (phenyl). The analysis made it possible to systematize the experimental observations and predict conditions of miscibility for aliphatic and semi-aromatic polyamides. The method was applied to a 1:1 composition of blends, and $\chi_{12}$ values were assumed to be temperature independent. Examples of the segmental interaction parameters, $\chi_{i j}$, used are given in Table 2.13. For binary mixtures of aliphatic polyamides $A_{x} B_{1-x}$ with $A_{y} B_{1-y}$ (where $A$ and $B$ groups were as defined above), the binary interaction parameter of the blend was expressed as $\chi_{12}=7.984(\mathrm{x}-\mathrm{y})^{2}$, a positive number; thus, these polymers are expected to be immiscible. The miscibility, if observed, may be explained by either the presence of hydrogen bonding or trans-reactions (transamidation) resulting in rapid homogenization of the system (e.g., PA-6/PA-46 at $310^{\circ} \mathrm{C}$ becomes homogenous in $4 \mathrm{~min}$ ) (Ellis 1992). 
Table 2.13 Segmental interaction parameters, $\chi_{i j}$, for polyamide blends (Ellis 1990b, 1993, 1995, 1997)

\begin{tabular}{|c|c|c|}
\hline Segment-1 & Segment-2 & $\chi_{12}$ \\
\hline$-\mathrm{CH}_{2-}$ & -NHCO- & $7.984 ; 8.534$ \\
\hline$-\mathrm{CH}_{2-}$ & $-\mathrm{CH}_{2}-\mathrm{NHCO}-\mathrm{CH}_{2}-$ & 1.479 \\
\hline$-\mathrm{CH}_{2}-$ & $-\mathrm{COO}-$ & 2.233 \\
\hline$-\mathrm{CH}_{2-}$ & $-\mathrm{C}_{6} \mathrm{H}_{4}-$ & $-0.288 ;-0.308$ \\
\hline$-\mathrm{CH}_{2}-$ & $-m \mathrm{C}_{6} \mathrm{H}_{4}-$ & 0.1 \\
\hline$-\mathrm{CH}_{2}-$ & $-\mathrm{NHCO}-\mathrm{C}_{6} \mathrm{H}_{4}-\mathrm{NHCO}-$ & 1.571 \\
\hline$-\mathrm{CH}_{2-}$ & $-\mathrm{NHCO}-p \mathrm{C}_{6} \mathrm{H}_{4}-\mathrm{NHCO}-$ & 1.680 \\
\hline$-\mathrm{CH}_{2-}$ & $-\mathrm{NHCO}-m \mathrm{C}_{6} \mathrm{H}_{4}-\mathrm{NHCO}-$ & 1.693 \\
\hline$-\mathrm{NHCO}-$ & $-\mathrm{C}_{6} \mathrm{H}_{4-}$ & $7.460 ; 7.974$ \\
\hline -NHCO- & $-\mathrm{COO}-$ & 3.880 \\
\hline$-\mathrm{NHCO}-$ & $-m \mathrm{C}_{6} \mathrm{H}_{4}-$ & 8.000 \\
\hline$-\mathrm{NHCO}-$ & $-\mathrm{NHCO}-\mathrm{C}_{6} \mathrm{H}_{4}-\mathrm{NHCO}-$ & 2.275 \\
\hline$-\mathrm{NHCO}-$ & $-\mathrm{NHCO}-p \mathrm{C}_{6} \mathrm{H}_{4}-\mathrm{NHCO}-$ & 2.432 \\
\hline$-\mathrm{NHCO}-$ & $-\mathrm{NHCO}-m \mathrm{C}_{6} \mathrm{H}_{4}-\mathrm{NHCO}-$ & 2.445 \\
\hline$-\mathrm{CH}_{2}-\mathrm{NHCO}-\mathrm{CH}_{2}-$ & $-\mathrm{NHCO}-\mathrm{C}_{6} \mathrm{H}_{4}-\mathrm{NHCO}-$ & -0.083 \\
\hline$-\mathrm{CH}_{2-}$ & $-\mathrm{COO}-$ & 2.233 \\
\hline$-\mathrm{NHCO}-$ & $-\mathrm{COO}-$ & 3.880 \\
\hline$-\mathrm{C}_{6} \mathrm{H}_{4-}$ & $-\mathrm{COO}-$ & 1.692 \\
\hline$-m \mathrm{C}_{6} \mathrm{H}_{4}-$ & $-\mathrm{COO}-$ & 1.500 \\
\hline$-m \mathrm{C}_{6} \mathrm{H}_{4-}$ & $-\mathrm{NHCO}-m \mathrm{C}_{6} \mathrm{H}_{4}-\mathrm{NHCO}-$ & 1.680 \\
\hline
\end{tabular}

It has been known since the early days that behavior of the aromatic polyamides (aramids) depends critically on the type of isomeric substitutions - para-substitutions result in crystalline, while meta-substitutions in amorphous polymers (Kwolek et al. 1962). Similarly, the two aramids: poly( $m$-xylene adipamide) and poly (hexamethylene isophthalamide), MXD6 and PA-6I, respectively, show different miscibility, e.g., with aliphatic polyamides. Clearly, blind application of the segmental interaction strategy to aromatic or semi-aromatic polyamides leads to conflicts. However, the problem can be resolved considering $p$ - and $m$-substituted phenyl as two different statistical segments (Ellis 1995). This idea is indeed evident in the segmental contributions listed in Table 2.14.

The segmental interaction values of $\chi_{i j}$ from Table 2.13 were used to compute the binary interaction parameters, $\chi_{12}$, for blends of a semi-aromatic polyamide, PA-3Me6T (Trogamid ${ }^{\mathrm{TM}} \mathrm{T}$ ) with aliphatic polyamides, PA-4 to PA-12. These values are listed in Table 2.14, along with the observations of miscibility, in good agreement (Ellis 1989). The data of Table 2.14 were used to construct Fig. 2.14. Similar to the case for the miscibility of halogenated polymers with polyesters, here also the miscibility depends on the $\mathrm{CH}_{2} / \mathrm{NHCO}$ ratio - with the best miscibility found when the group ratio was 7, e.g., for PA-410.

Later, this analysis was extended to PA blends with polyesters (Ellis 1993, 1995; 1997). Thus, in the simplest case of PA blends with aliphatic polyesters, i.e., blends of $\mathrm{A}_{\mathrm{x}} \mathrm{B}_{1-\mathrm{x}}$ with $\mathrm{A}_{\mathrm{y}} \mathrm{D}_{1-\mathrm{y}}$, where the units are $\mathrm{A},-\mathrm{CH}_{2}-\mathrm{B},-\mathrm{NHCO}-$; and 
Table 2.14 Calculated binary interaction parameters, $\chi_{12}$, based on segmental contributions values (from Table 2.13) for blends of aliphatic polyamides with PA-3Me6T and experimental observation of miscibility (Ellis 1989)

\begin{tabular}{llrl}
\hline Aliphatic PA & Aromatic PA & \multicolumn{1}{c}{$\chi_{12}$} & Observation \\
\hline PA-4 & PA-3Me6T & 0.0400 & Immiscible \\
\hline PA-46 & PA-3Me6T & 0.0065 & Immiscible \\
\hline PA-6 & PA-3Me6T & -0.0050 & Miscible \\
\hline PA-66 & PA-3Me6T & -0.0050 & Miscible \\
\hline PA-69 & PA-3Me6T & -0.0069 & Miscible \\
\hline PA-610 & PA-3Me6T & -0.0058 & Miscible \\
\hline PA-612 & PA-3Me6T & -0.0023 & Miscible \\
\hline PA-11 & PA-3Me6T & 0.0061 & Immiscible \\
\hline PA-12 & PA-3Me6T & 0.0104 & Immiscible \\
\hline
\end{tabular}

Fig. 2.14 The calculated binary interaction parameter for blends of PA-3Me6T with aliphatic polyamides as a function of the $-\mathrm{CH}_{2}-$ - $-\mathrm{NHCO}-$ group ratio of the aliphatic polyamide (Data from Ellis 1989)

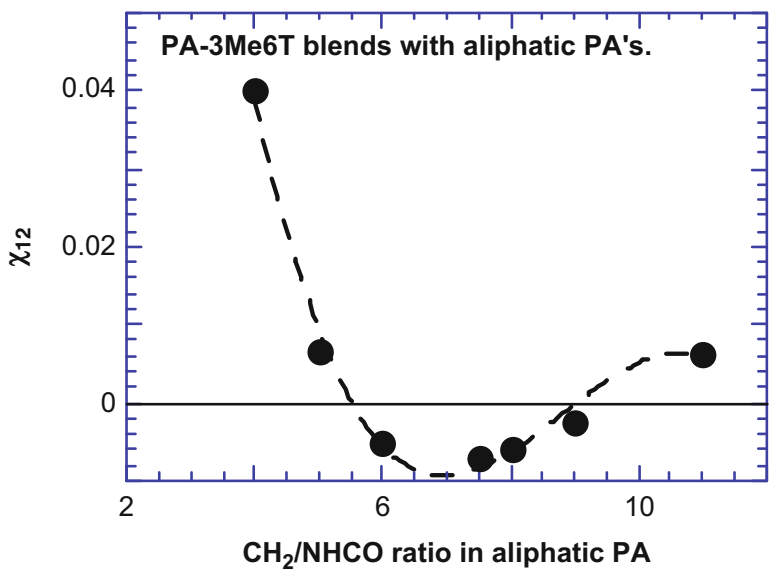

$\mathrm{D},-\mathrm{COO}-$ (ester) and the subscripts $\mathrm{x}, \mathrm{y}$ refer to the polymers' mer volume fractions, the binary $\chi$ based on segmental contributions is (Ellis 1993) $\chi_{12}=(\mathrm{y}-\mathrm{x})(1-\mathrm{x}) \chi_{A B}+(\mathrm{x}-\mathrm{y})(1-\mathrm{y}) \chi_{A D}+(1-\mathrm{x})(1-\mathrm{y}) \chi_{B D}$. The values of $\chi_{i j}$ are also listed in Table 2.13. A similar principle was used to apply the LCT to polyolefin copolymer blends (Freed and Dudowicz 2005).

After 30 years since its conception, the heat of mixing (or analog calorimetry) method for the determination of polymer/polymer miscibility is becoming increasingly sophisticated. The low molecular weight analogs are selected on the basis of detailed calculations of the electrostatic charges on the atoms and molecules, using molecular orbital theories. The following principles have been formulated (Sandler and Wu 1991; Ziaee and Paul 1996; 1997):

The geometry of a functional group (in the polymer and selected analog molecule) should be the same.

The electrostatic charge of each atom in a functional group should be approximately the same. 
Table 2.15 Segmental binary interaction parameters, $B_{i j}$, at 0.5 volume fraction, for polar groups related to bisphenol-A carbonates and acrylonitriles (Ziaee and Paul 1996, 1997)

\begin{tabular}{|c|c|c|c|}
\hline Group-1 & Group-2 & $T\left({ }^{\circ} \mathrm{C}\right)$ & $B_{i j}(\mathrm{cal} / \mathrm{mL})$ \\
\hline$-\mathrm{C}_{6} \mathrm{H}_{5}$ & $-\mathrm{CH}_{2-}$ & 30 & $8.74 \pm 0.16$ \\
\hline$-\mathrm{C}_{6} \mathrm{H}_{5}$ & $-\mathrm{CH}_{2-}$ & 80 & $7.74 \pm 0.16$ \\
\hline$-\mathrm{C}_{6} \mathrm{H}_{5}$ & $-\mathrm{CH}_{2-}$ & 130 & $5.93 \pm 0.20$ \\
\hline$-\mathrm{C}_{6} \mathrm{H}_{5}$ & $\mathrm{C}_{6} \mathrm{H}_{5}-\mathrm{O}-$ & 80 & $-2.11 \pm 0.30$ \\
\hline$-\mathrm{CH}_{2-}$ & $\mathrm{C}_{6} \mathrm{H}_{5}-\mathrm{O}-$ & 80 & $5.44 \pm 0.15$ \\
\hline$-\mathrm{C}_{6} \mathrm{H}_{4}-\mathrm{O}-$ & $\mathrm{C}_{6} \mathrm{H}_{5-}$ & 80 & $-0.60 \pm 0.45$ \\
\hline$-\mathrm{C}_{6} \mathrm{H}_{4}-\mathrm{O}-$ & $-\mathrm{CH}_{2-}$ & 80 & $7.24 \pm 0.26$ \\
\hline$-\mathrm{C}_{6} \mathrm{H}_{5}$ & $-m \mathrm{XO}-$ & 80 & $-0.91 \pm 0.14$ \\
\hline$-\mathrm{CH}_{2-}$ & $-m \mathrm{XO}-$ & 80 & $3.13 \pm 0.18$ \\
\hline$-\mathrm{C}_{6} \mathrm{H}_{5}$ & $-\mathrm{CO}-$ & 80 & $-36.9 \pm 18$ \\
\hline$-\mathrm{C}_{6} \mathrm{H}_{5}$ & $-\mathrm{CO}-$ & 90 & 15.1 \\
\hline$-\mathrm{CH}_{2-}$ & $-\mathrm{CO}-$ & 80 & $-19.1 \pm 15$ \\
\hline$-\mathrm{CH}_{2-}$ & $-\mathrm{CO}-$ & 90 & 41.4 \\
\hline $\mathrm{C}_{6} \mathrm{H}_{5}-\mathrm{O}-$ & $-\mathrm{CO}-$ & 90 & -8.97 \\
\hline$-\mathrm{C}_{6} \mathrm{H}_{5}$ & $-\mathrm{Ph}_{2} \mathrm{OCOO}-$ & 90 & $0.55 \pm 0.21$ \\
\hline$-\mathrm{CH}_{2-}$ & $-\mathrm{Ph}_{2} \mathrm{OCOO}-$ & 90 & $10.1 \pm 0.2$ \\
\hline$-\mathrm{C}_{6} \mathrm{H}_{5}$ & $-m \mathrm{X}_{2} \mathrm{OCOO}-$ & 130 & $0.40 \pm 0.06$ \\
\hline$-\mathrm{CH}_{2}-$ & $-m \mathrm{X}_{2} \mathrm{OCOO}-$ & 130 & $4.56 \pm 0.06$ \\
\hline$-\mathrm{C}_{6} \mathrm{H}_{5}$ & $-\mathrm{CH}_{2}-\mathrm{CN}$ & 30 & $19.1 \pm 0.2$ \\
\hline$-\mathrm{CH}_{2-}$ & $-\mathrm{CH}_{2}-\mathrm{CN}$ & 30 & $60.8 \pm 0.5$ \\
\hline
\end{tabular}

Notes: Most groups are the obvious common compounds (methylene, $-\mathrm{CH}_{2}-$ (cf. alkyl); phenyl, $-\mathrm{C}_{6} \mathrm{H}_{5}$; carbonyl, $-\mathrm{CO}-$; phenol, $\mathrm{C}_{6} \mathrm{H}_{5}-\mathrm{O}-$; etc.). The rest of the groups relate to the segments of bisphenol-A polycarbonate and tetramethyl bisphenol-A polycarbonate: $m \mathrm{X}$ is $m$-xylyl: $-\mathrm{C}_{6} \mathrm{H}_{2}\left(\mathrm{CH}_{3}\right)_{2}-$ (cf. di(2,6-dimethyl)phenyl); $m$ XO, $m$-xylyl ether: $-\mathrm{C}_{6} \mathrm{H}_{2}\left(\mathrm{CH}_{3}\right)_{2}-\mathrm{O}$-; $\mathrm{Ph}_{2} \mathrm{OCOO}$, diphenyl carbonate: $-\mathrm{C}_{6} \mathrm{H}_{4}-\mathrm{O}-\mathrm{CO}-\mathrm{O}-\mathrm{C}_{6} \mathrm{H}_{4-} ; \quad m \mathrm{X}_{2} \mathrm{OCOO}$, di $(m$-xylyl $)$ carbonate, viz., di (2,6-dimethyl)phenyl carbonate, $-\left[\mathrm{C}_{6} \mathrm{H}_{2}\left(\mathrm{CH}_{3}\right)_{2}\right]-\mathrm{O}-\mathrm{CO}-\mathrm{O}-\left[\mathrm{C}_{6} \mathrm{H}_{2}\left(\mathrm{CH}_{3}\right)_{2}\right]-$, i.e., a diphenyl carbonate with each of the two phenyls being a 2,6-dimethyl substituted phenyl ( $m$-xylyl)

The functional group should be approximately electroneutral.

The functional group should be the smallest entity, identified by dividing the analog molecule into a collection of electroneutral groups.

Going beyond effectively apolar polymers - those whose miscibility is determined by van der Walls interactions (dispersion forces) - for polar polymer, the partial charges can also be accounted for and incorporated in segmental binary interaction parameters. A careful analysis of such data makes it possible to determine binary interaction parameters, $B_{i j}$, as those listed in Table 2.15. For these data, the authors calculated the (enthalpic) binary interaction parameters for several polymer/oligomer pairs, from Eq. 2.49 using the $B_{i j}$ values, where the heat of mixing was determined at $T=80^{\circ} \mathrm{C}$ for $1: 1 \mathrm{blend} / \mathrm{mixure}$ compositions (Ziaee and Paul 1996; 1997). The calculated values were in good agreement with the measured ones, based mostly on polystyrene blends with bisphenol-A polycarbonate and tetramethyl bisphenol-A polycarbonate, as well as with acrylonitrile-containing polymers. 
In summary, the heat of mixing approach and the corresponding segmental binary interaction parameters entail several simplifying assumptions. The numerical values of the parameters do vary depending on method of evaluation, selected system/pair, concentration, temperature, etc. However, the method has been found useful for the identification of potentially miscible systems and conditions. Furthermore, the approach provided a valid tool for interpretation of the blends' behavior at higher concentration, viz., 1:1 compositions, where the interaction parameters are relatively insensitive to the variability of concentration. Under these conditions, the segmental binary interaction parameters have been successfully employed to describe:

Miscibility in systems without strong specific interactions

Behavior of blends of a homopolymer with copolymer

Miscibility of polymer series (chemically similar polymers, e.g., polyamides, polycarbonates)

Window of miscibility in two- or three-component systems

Chimney of miscibility in two- or three-component systems

\subsubsection{Solubility Parameter Approach}

The concept of the solubility parameter originates from Hildebrand's work on enthalpy of regular solutions (Hildebrand and Scott 1950, 1962; Shinoda 1978) and was defined as the square root of the cohesive energy density $(\delta \equiv \sqrt{E / V})$. Accordingly, in a strict sense, the molecular interactions should be nonspecific, without forming associations or orientation, hence not polar or hydrogen-bonding interactions. Another fundamental assumption was that the intermolecular interactions 1-2 are geometric mean of the intramolecular interactions, 1-1 and 2-2:

$$
\begin{aligned}
& \Delta H_{m} \approx \Delta G_{m}^{\text {non comb }}=\phi_{1} \phi_{2} V_{m}\left(\delta_{1}-\delta_{2}\right)^{2} \geq 0 \\
& \Delta H_{m} \approx \phi_{1} \phi_{2} V_{m}\left[\sqrt{\frac{E_{1}^{V}}{V_{1}}}-\sqrt{\frac{E_{2}^{V}}{V_{2}}}\right]^{2}=\left[\frac{E_{1}^{V}}{V_{1}}-\frac{2 E_{12}^{V}}{\sqrt{V_{1} V_{2}}}+\frac{E_{2}^{V}}{V_{2}}\right]
\end{aligned}
$$

where $E_{i}^{V}$ is the molar energy of vaporization of substance $i$ and $\delta_{i}$ is its solubility parameter. Comparing Eq. 2.51 to Eq. 2.35 makes it evident that the binary interaction parameter chi can be written in solubility parameter terms:

$$
\chi_{12}^{\prime} \approx\left(V_{r e f} / R T\right)\left(\delta_{1}-\delta_{2}\right)^{2}
$$

where the reference volume is usually taken as $V_{\text {ref }}=100 \mathrm{~mL} / \mathrm{mol}$ (viz., the liquid density is 1). It is important to note that, as stated by Eq. 2.51, the above interaction parameter is limited to the enthalpic part of binary interaction parameter, i.e.,

$$
\chi_{12}^{\prime}=\chi_{S}+\chi_{H}=\chi_{S}+\left(V_{r e f} / R T\right)\left(\delta_{1}-\delta_{2}\right)^{2}
$$

The entropic term in Eq. $2.53, \chi_{S}$, originates from local configurational effects, as well as combinatorial entropy contributions. When Eq. 2.52 is used, then $\chi_{S}$ must be accounted for through other means. 
For molecules without polar groups, the solubility parameter $\delta_{i}$ may be determined or approximated:

1. From the $\delta_{i}$ definition (see Eq. 2.51):

$$
\delta_{i}^{2}=E_{i}^{V} / V=\left(\Delta H_{i}^{V}-P V\right) / V \approx\left(\Delta H_{i}^{V}-R T\right) / V
$$

2. From empirical correlation, as, for example, with the surface tension coefficient $\left(\gamma_{i}\right)$ or the dipole moment $\left(\mu_{i}\right)$ :

$$
\delta_{i}^{2}=14 \gamma_{i} / V^{1 / 3} \text { or } \delta_{i}=37.4 \mu_{i} / V^{1 / 2}
$$

3. By solving Eq. 2.51 for $\delta_{1}$, knowing the experimental values of $\Delta H_{m}$ for material 1 in a series of solvents with known values of $\delta_{2}$

For small molecules without strong interactions, the values of the solubility parameter vary from 5.9 (for $\mathrm{C}_{6} \mathrm{~F}_{14}$ ) to 14.1 (for $\mathrm{I}_{2}$ ), whereas it is about 30 for $\mathrm{H}_{2} \mathrm{O}$ (Hansen 2000); the standard error of these estimates is \pm 0.2 , much larger for water. Given that polymer (melt) evaporation experiments are impossible, the solubility parameter of a polymer is usually determined by measurements of its oligomeric liquids or by indirect measurements of its behavior in a solvent of known solubility parameter. The solvent approach allows for the polymer to be cross-linked (the degree of swelling $\left(D_{s}\right)$ is measured) or simply dissolved in the solvent (the intrinsic viscosity ([ $\eta]$ ) is usually measured). From the plot of either $\mathrm{D}_{\mathrm{s}}$ or $[\eta]$ versus $\delta_{\text {solvent }}$, the value of $\delta_{\text {polymer }}$ is determined as the value that corresponds to the peak location (Utracki 1972), or by appropriately fitting the whole curve (Hansen 2000). For copolymers, the volume additivity of the monomeric solubility parameters is assumed, i.e., $\delta=\sum \phi_{i} \delta_{i}$. This assumption also follows from the group-contribution method used to compute $\delta$ from the chemical and structural characteristics of polymeric chain, vide infra (Grulke 1989; van Krevelen 1992; Coleman et al. 1992, 2006). Correlation between the solubility parameter theory and the EoS based on the Flory model was also explored (Biros et al. 1971). The authors demonstrated that $\delta=P^{* 1 / 2} V^{*} / V$, where $P^{*}$ and $V^{*}$ are the pressure-reducing and volume-reducing parameters, respectively.

Given the definition of $\delta$, the temperature and pressure gradients of the solubility parameter can be approximated by

$$
\left.\frac{\partial \ln \delta}{\partial T}\right|_{P} \cong-\alpha_{P} \quad \text { and }\left.\quad \frac{\partial \ln \delta}{\partial P}\right|_{T} \cong+\beta_{T}
$$

where $\alpha_{P}$ is the isobaric thermal expansion coefficient and $\beta_{T}$ is the isothermal compressibility. These relations can be used to correct/extrapolate the value of $\delta$ to any temperature and pressure of interest ( $\delta$ values are usually given at $298 \mathrm{~K}$ and ambient pressure).

For completeness of the above discussion, a few examples of solubility parameters for selected polymers are given in Table 2.16. More extensive listings can be found in the source literature (Shinoda 1978; Van Krevelen 1976; Grulke 1989; 
Table 2.16 Solubility parameters, experimental and calculated, for various common polymers at $298 \mathrm{~K}$. All $\delta$ values are in $(\mathrm{J} / \mathrm{mL})^{1 / 2}$ the calculated values have a nominal error of $\pm 0.8(\mathrm{~J} / \mathrm{mL})^{1 / 2}$

\begin{tabular}{lllll}
\hline Polymer & Experimental $\delta$ & & & Calculated $\delta$ \\
\hline PTFE & Shinoda 1978 & van Krevelen 1976 & Grulke 1989 & Coleman 1990 \\
\hline PDMS & 12.69 & 12.7 & - & - \\
\hline PE & 14.94 & - & $14.90-15.59$ & - \\
\hline PP & 16.17 & 15.8 & $15.76-17.09$ & 16.4 \\
\hline PPG & - & $16.8-18.8$ & $18.80-19.20$ & 15.2 \\
\hline PIB & 16.58 & $15.4-20.3$ & 19.20 & 17.4 \\
\hline PS & 18.63 & $16.0-16.6$ & $14.50-16.47$ & 14.8 \\
\hline PVAc & 19.24 & $17.4-19.0$ & $17.40-19.80$ & 19.5 \\
\hline PMMA & 19.44 & $19.1-22.6$ & $18.00-22.61$ & 19.7 \\
\hline PVC & 19.65 & $18.6-26.2$ & $18.58-26.27$ & 18.5 \\
\hline PET & 21.90 & $19.2-22.1$ & $19.20-22.10$ & 20.3 \\
\hline PMAN & 21.90 & $19.9-21.9$ & 21.54 & 23.6 \\
\hline CA & 22.31 & 21.9 & - & 24.4 \\
\hline PA-66 & 27.84 & - & $22.30-23.22$ & - \\
\hline PAN & 31.52 & 27.8 & $22.87-23.37$ & - \\
\hline
\end{tabular}

Note: The calculated values of $\delta$ in the last column can be reproduced by the CD-ROM tool provided in (Coleman and Painter 2006a, b). Although the tool allows for the input of almost any arbitrary polymer, and even provides a calculated $\delta$ value for these, care must be given to the uncertainty associated with certain groups' contributions, e.g., $-\mathrm{CF}_{2}-,>\mathrm{Si}<$, etc., whose attraction values are denoted as "rough estimates"; rather inaccurate numbers for $\delta$ are obtained for polymers that are comprised primarily by such groups, e.g., using $-\mathrm{CF}_{2}-$ group contributions for PTFE above would result in $\delta=10.2$, but this value is with \pm 5 uncertainty. Calculated $\delta$ 's with large uncertainties are omitted above. These limitation are outlined in the accompanying booklet (Coleman and Painter 2006a, b)

Coleman et al. 1990). As evident, by comparing data from different studies, cf. Table 2.16, one complication of the solubility parameter method is poor reproducibility of the measured values. Selection of different commercial polymers and solvents, or using different sets of solvents, may significantly change the value of the measured $\delta$. Also, more recent and arguably more accurate calculated $\delta$ values exist (Hansen 2000; Coleman and Painter 2006a, b), vide infra.

The biggest drawback of the solubility parameter approach, as described above, is the omission of the entropic and specific interactions' effects. Furthermore, the fundamental dependencies do not take into account either the structural (isomeric), orientation, or the neighboring group effects (e.g., steric effects, intermolecular screening, functional group accessibility) (Coleman and Painter 2006a, b). However, solubility parameters can provide a guide toward miscibility: Since the contributions that are included in the solubility parameter calculation are indeed detrimental to miscibility, minimizing their value must but help the miscibility.

In the simplest approach, the solubility parameter of a polymer can be calculated by a summation of group contributions (Coleman and Painter 1988, 1989, 1990, $2006 a, b)$. The essence of this approach is to assume pairwise additivity for the interaction of sub-monomeric building blocks, "groups," which can be added to 
Table 2.17 Selected group contributions for the calculation of solubility parameters based on Eq. 2.57; molar volume $V_{i}^{g}\left[\mathrm{~cm}^{3} /\right.$ mole $]$ and molar attraction $F_{i}^{g}\left[\left(\mathrm{cal} \cdot \mathrm{cm}^{3}\right)^{1 / 2} / \mathrm{mole}\right]($ Coleman and Painter 2006a, b). The source contains additional groups and important instructions of how, and when, meaningful solubility parameters for polymers can be obtained

\begin{tabular}{lrr}
\hline Group & \multicolumn{1}{c}{$V_{i}^{g}$} & \multicolumn{1}{c}{$F_{i}^{g}$} \\
\hline$-\mathrm{CH}_{3}$ & 31.8 & 218 \\
\hline$-\mathrm{CH}_{2}-$ & 16.5 & 132 \\
\hline$>\mathrm{CH}-$ & 1.9 & 23 \\
$>\mathrm{C}<$ & -14.8 & -97 \\
\hline $\mathrm{C}_{6} \mathrm{H}_{3}-$ & 41.4 & 562 \\
\hline$-\mathrm{C}_{6} \mathrm{H}_{4}-$ & 58.5 & 652 \\
\hline$-\mathrm{C}_{6} \mathrm{H}_{5}$ & 75.5 & 735 \\
\hline$-\mathrm{CH}_{2}$ & 29.7 & 203 \\
\hline$-\mathrm{CH}=$ & 13.7 & 113 \\
\hline$>\mathrm{C}=$ & -2.4 & 18 \\
\hline$-\mathrm{OCO}-$ & 19.6 & 298 \\
\hline$-\mathrm{CO}-$ & 10.7 & 262 \\
\hline$-\mathrm{O}-$ & 5.1 & 95 \\
\hline$-\mathrm{Cl}$ & 23.9 & 264 \\
\hline$-\mathrm{CN}$ & 23.6 & 426 \\
\hline$-\mathrm{NH} \mathrm{H}_{2}$ & 18.6 & 275 \\
\hline$>\mathrm{NH}$ & 8.5 & 143 \\
\hline$>\mathrm{N}-$ & -5.0 & -3 \\
\hline
\end{tabular}

form the monomer units. For example, a simple hydrocarbon, such as $n$-octane, is assumed to consist of six $-\mathrm{CH}_{2}-$ and two $-\mathrm{CH}_{3}$ groups; using the energy of vaporization for a series of such paraffins with varied length, Coleman and Painter estimated molar attraction constants for $-\mathrm{CH}_{2}-$ and $-\mathrm{CH}_{3}$.

Further, by including branched hydrocarbons, and molecules containing other functional groups (ether oxygens, esters, nitriles, etc.), a table of constants was obtained (Table 2.17) and, subsequently, used to calculate the solubility parameter for various polymers, using the relationship:

$$
\delta=\frac{\sum_{i} F_{i}^{g}}{\sum_{i} V_{i}^{g}}
$$

In the late 1980s, a new approach to the solubility parameter concept was developed (Painter et al. 1988, 1989a, b, 1990, Coleman et al. 1988, 1989, 1990, 1991, 1995), which was later further refined to address some of the drawbacks mentioned above (Coleman and Painter 2006a, b). The authors start by recasting Flory-Huggins Eq. 2.35 into the form (Painter et al. 1988):

$$
\frac{\Delta G_{m}}{R T}=\frac{V}{V_{\text {ref }}}\left[\frac{\phi_{1}}{N_{1}} \ln \phi+\frac{\phi_{2}}{N_{2}} \ln \phi_{2}+\chi_{12}^{\prime} \phi_{1} \phi_{2}\right]+\frac{\Delta G_{H}}{R T}
$$


where $V$ is the total molar volume of the two components and $V_{r e f}=100 \mathrm{~mL} / \mathrm{mol}$ is the reference volume. Originally, the new term, $\Delta G_{H}$, was introduced to express the effects of hydrogen bonding in blends (where one polymer self-associates, the other does not - but it is capable to hydrogen bond to the first one). Since then, this term has been extended to describe all specific interactions (hydrogen bonding, ion-ion, ion-dipole, charge transfer, $\pi-\pi$ electron interactions, etc.) that provide negative contributions to the free energy of mixing. Equation $2.35 \mathrm{~b}$ distinguishes three contributions to the free energy of mixing: the configurational entropy (given by the two logarithmic terms), the dispersive or van der Waals interactions (given by the positive $\chi_{12}^{\prime} \phi_{1} \phi_{2}$ term), and the strong interaction term, $\Delta G_{H}$. For systems with no specific interactions $\left(\Delta G_{H} \approx 0\right)$, Eq. $2.35 \mathrm{~b}$ becomes the FH equation. The interactions of the van der Waals type are accounted for by the $\chi_{12}^{\prime} \phi_{1} \phi_{2} \geq 0$ term, with $\chi_{12}^{\prime}$ quantified through Hildebrand's solubility parameters via Eq. 2.52. The degree of polymerization dependence of miscibility was assumed to follow Eq. 2.36.

The novelty of the Coleman-Painter theory is the calculation of the hydrogenbonding contributions in the free energy of mixing (Painter et al. 1988, 1995, 2000). Previous attempts to describe blends with specific interactions, including hydrogen bonds, usually employed the FH theory and allowed the $\chi$ parameter to become negative. However, such an approach is rather unsatisfactory because:

1. The hydrogen-bonded contacts are not random (i.e., the interaction term cannot take the usual $\chi \phi_{1} \phi_{2}$ form, because only for strictly random mixing the term $\phi_{1} \phi_{2}$ provide the probability of a 1-2 contact).

2. The formation of hydrogen bonds results in a high loss of degrees of rotational freedom in the molecules or segments involved and, hence, introduces significant entropic as well as enthalpic changes in $\Delta G_{m}$.

3. It is rather unreasonable to lump both specific and nonspecific interactions into one overall interaction parameter, not only because they are very different in character (composition dependence, temperature dependence, etc.), but also because it is often the balance between the two, i.e., specific and nonspecific interactions, that determines the blend phase behavior.

In contrast, the Coleman-Painter theory quantified the hydrogen-bonding contributions implicitly by re-enumerating an "equilibrium distribution" of the various species in the mixture after accounting for hydrogen-bonding formation (in pairs, hydrogen-bonded dimers, or longer sequences $h$-mers). Specifically, starting from Eq. $2.35 \mathrm{~b}$, the final result for the specific interaction term $\Delta G_{H}$ can be written as

$$
\begin{aligned}
\frac{\Delta G_{H}}{R T}= & \frac{\phi_{1}}{r} \ln \phi_{01}+\phi_{2} \ln \frac{\phi_{21}}{\phi_{21}^{0}} \\
& +K \phi_{2}\left(\phi_{21}-\phi_{21}^{0}\right)+\phi_{2}\left(1-K \phi_{21}\right) \frac{X}{1+X} \\
& -\left[\frac{\phi_{2}}{n_{H}^{0}} \ln \phi_{2}+\frac{\phi_{1}}{r} \ln \phi_{1}\right]
\end{aligned}
$$


with

$$
\left.r=V_{1} / V_{2} \quad \text { (equiv. } V_{r e f}=V_{2}\right) \quad \text { and } \quad X=K_{1} \phi_{01} / r
$$

where the volume fractions $\phi_{1}$ and $\phi_{2}$ are the usual volume fraction of polymer 1 and 2, respectively, in the blend; $\phi_{01}$ is the volume fraction of polymer 1 that remains unassociated; $\phi_{21}$ and $\phi_{21}^{0}$ represent the nonbonded monomers of polymer 2 in the blend and in the neat state, respectively; and $n_{H}^{0}$ is the equilibrium length of the hydrogen-bonded sequence of monomers. Note that a term describing the energy of hydrogen bond formation does not appear explicitly in the result (Eq. 2.58). To compute the thermodynamic contribution of the strong interactions to the overall thermodynamic behavior of a blend, one must first determine the three principal constants: $K_{A}, K_{B}$, and $K_{2}$; these are defined as association equilibrium constants for the formation of hydrogen bond between $B$ and $A$ units (of polymer 2 and 1), respectively, the formation of self-association between sequences of multiple B units, and the formation of doublets of the hydrogen-bonded B units (Coleman and Painter 1995; Painter and Coleman 2000). The $K_{A}, K_{B}$, and $K_{2}$ can be quantified experimentally, e.g., by IR spectroscopy, they are interrelated (only two of the three $K$ s need to be determined independently) and are constrained by stoichiometry (the total number of $i$-type mers is the sum of the associated and non-associated mers); the fraction of hydrogen-bonded $A$ groups, $f_{A}^{H B}$ which can be determined experimentally, can yield

$$
\begin{aligned}
& f_{A}^{H B}=1-\frac{\phi_{0 A}}{\phi_{A}}=1-\left[\frac{1}{1+K_{A} \phi_{B} \Gamma_{1}}\right] \quad \text { with } \Gamma_{1}=1-\frac{K_{2}}{K_{B}}+\frac{K_{2} / K_{B}}{1-K_{B} \phi_{0 B}} \\
& \text { and } \begin{aligned}
\phi_{B} & =\phi_{0 B} \Gamma_{2}\left[1+\frac{K_{A} \phi_{0 A}}{r}\right] \quad \text { with } \Gamma_{2}=1-\frac{K_{2}}{K_{B}}+\frac{K_{2} / K_{B}}{\left(1-K_{B} \phi_{0 B}\right)^{2}}
\end{aligned}
\end{aligned}
$$

For the computation of an isobaric phase diagram, the temperature dependence of the association constants has to be known: $K_{A}(T), K_{B}(T)$, and $K_{2}(T)$; the polymerspecific $T$ dependence follows an Arrhenius-type dependence:

$$
K_{i}=K_{i}^{o} \exp \left(\frac{-h_{i}}{R}\left[\frac{1}{T}-\frac{1}{T^{o}}\right]\right)
$$

where $h_{i}$ is the enthalpy of formation of a hydrogen bond, which can be determined from experimental data (Painter and Coleman 2000). When the pressure influence on blend miscibility is of interest, the $K_{i}$ functions must be evaluated within the appropriate range of pressures as well: $K_{A}(T, P), K_{B}(T, P)$, and $K_{2}(T, P)$.

As mentioned, one of the strong points of this theory is that, since the three equilibrium association constants are defined in terms of chemical repeating units, these constants can, thus, be measured by spectroscopic means (Painter et al. 1989a, b, 2000). Infrared spectroscopy is sensitive to hydrogen bonding, but selection of the 
Table 2.18 Association equilibrium constants for polyvinylphenol $(\mathrm{PVPh})$, blends at $25{ }^{\circ} \mathrm{C}$ (Coleman et al. 1989; Xu et al. 1991)

\begin{tabular}{llrrrr}
\hline Polymer & & \multicolumn{1}{c}{$N$} & \multicolumn{1}{c}{$K_{A}$} & \multicolumn{1}{c}{$K_{B}$} & \multicolumn{1}{c}{$K_{2}$} \\
\hline PVPh & & 60 & 37.1 & 66.8 & \multicolumn{1}{c}{1.0} \\
\hline StVPh & {$[75 \% \mathrm{VPh}]$} & 371 & 27.5 & 49.6 & 15.6 \\
\hline StVPh & {$[43 \% \mathrm{VPh}]$} & 223 & 16.5 & 29.8 & 9.4 \\
\hline StVPh & {$[25 \% \mathrm{VPh}]$} & 131 & 9.7 & 17.5 & 5.5 \\
\hline StVPh & {$[8 \% \mathrm{VPh}]$} & 37 & 2.8 & 5.0 & 1.6 \\
\hline StVPh & {$[2 \% \mathrm{VPh}]$} & 11 & 0.8 & 1.4 & 0.5 \\
\hline PMA & & 350 & 53.2 & - & - \\
\hline PEA & & 700 & 46.8 & - & - \\
\hline PVAc & & 3,000 & 64.0 & - & - \\
\hline EVAc & {$[70 \% \mathrm{VAc}]$} & 3,000 & 61.6 & - & - \\
\hline PCL & & 3,000 & 66.2 & - & - \\
\hline$h_{i}(\mathrm{kcal} / \mathrm{mol})$ & & - & 3.8 & 5.2 & 5.6 \\
\hline
\end{tabular}

Note: $\mathrm{StVPh}[x \% \mathrm{VPh}]$ stands for poly(styrene-co-vinylphenol) with $x \%$ of vinylphenol comonomer

most appropriate vibration band is essential. For example, instead of $\mathrm{N}-\mathrm{H}$ or $\mathrm{O}-\mathrm{H}$ stretching, the carbonyl group $(\mathrm{C}=\mathrm{O})$ may be a better choice. Examples of the association constants for blends of poly(4-vinyl phenol), $\mathrm{PVPh}$, with acrylic polymers are given in Table 2.18. These values were used to compute the phase diagrams for blends of styrene-co-vinyl phenol (comprising of 2-100\% VPh), with either acrylic polymers, poly(alkyl ${ }_{i}=1 . .6$-methacrylates), or polyethers (Serman et al. 1989, 1991; $\mathrm{Xu}$ et al. 1991). The experimental data confirmed the theoretical prediction, thus corroborating the validity of the model. Since then, more blends, as well as polymer solutions, have been investigated experimentally and compared favorably against the theoretical predictions (Painter et al. 2000).

As stated before, the Hildebrand solubility parameter concept was developed for nonpolar, low molecular weight liquids at room temperature. For polar molecules, the method did not provide consistent information. To avoid trouble, initially all liquids were divided into three categories for poorly, moderately, and strongly interacting systems. Another route was taken by Hansen (1967) who postulated that all intermolecular forces:

- London dispersion forces between nonpolar molecules

- Repulsive forces between nonpolar molecules

- Coulombic ion/ion interactions

- Dipole/dipole interactions between the permanent dipoles

- Permanent dipole/ion interactions

- Induced dipole/ion interactions

- Permanent dipole/induced dipole interactions

- Charge-transfer forces

- Hydrogen bonding

- Coordination bonding

- Metallic bonding, etc. 
Fig. 2.15 Schematic representation of Hansen's miscibility sphere, Eq. 2.60

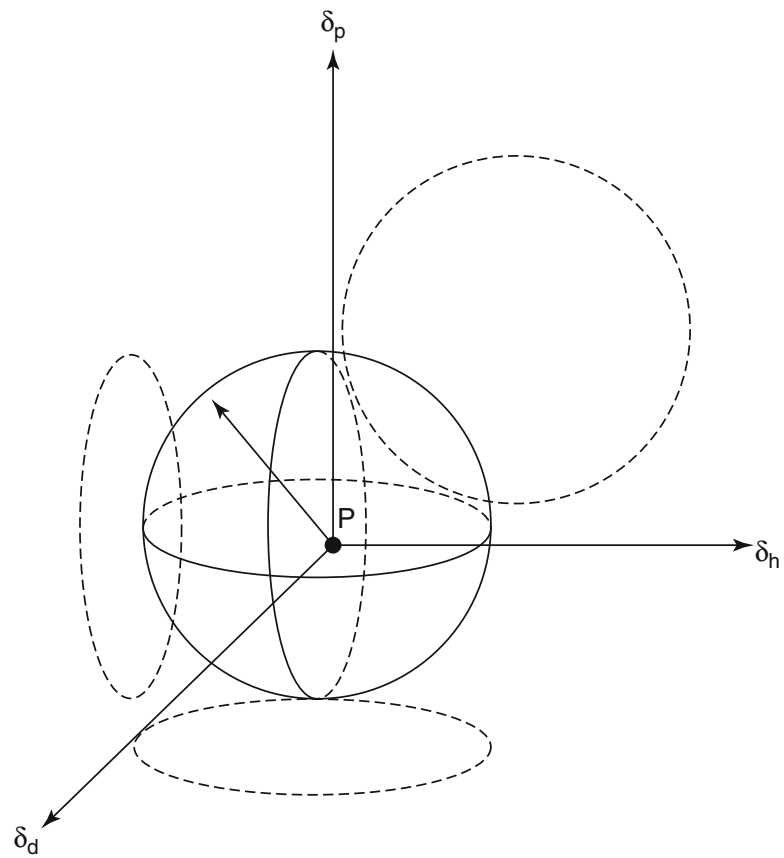

can be combined and grouped into three types of interactions, dispersive, polar, and hydrogen bonding, and a substance's total solubility parameter can thus be written as

$$
\delta_{i}^{2}=\delta_{i d}^{2}+\delta_{i p}^{2}+\delta_{i h}^{2}
$$

where the subscripts $d, p$, and $h$ represent the dispersive, polar, and hydrogenbonding interactions, respectively. Accordingly, two substances would be miscible only when their solubility parameters place them within the critical radius of a spheroid, defined as (Hansen 1967, 1995)

$$
R_{12}^{2} \mathrm{crit}=\Theta\left(\delta_{1 d}-\delta_{2 d}\right)^{2}+\left(\delta_{1 p}-\delta_{2 p}\right)^{2}+\left(\delta_{1 h}-\delta_{2 h}\right)^{2}=\chi_{12} \geq 0
$$

where the semiempirical fudge parameter: $\Theta$ assumes values of 4-5 and accounts for the dominant role that the dispersion forces play in binary solubility. The concept is illustrated in Fig. 2.15. Examples of the numerical value of the Hansen's parameters are given in Table 2.16 (Hansen 1967, 1994, 1995; Hansen and Beerbower 1971; Grulke 1989; Luciani et al. 1996b), whereas a comprehensive collection of values has been compiled in a handbook (Hansen 2000). As reported by Hansen $(1995,2000)$, values of these parameters may greatly vary from one commercial polymer resin to another, reflecting diversity of molecular weights, 
molecular weight distribution, the presence or absence of catalyst, and a great diversity of additives (caution is advised for their use). The values of the solubility parameters for solvents are considered more dependable.

Much like the Coleman-Painter approach, the solubility method also allows for the values of the Hansen's partial solubility parameters, $\delta_{i d}, \delta_{i p}$, and $\delta_{i h}$, to be calculated from the molecular structure of a polymer by using additive group contributions. van Krevelen (1976) demonstrated usefulness of the method using contributions from individual atoms, structural groups, and configurations. However, care must be given when such solubilities are employed in mixture that contain dissimilar in interactions polymers. For example, comparing to the calculations of interfacial surface tensions based on dispersive and polar components, it becomes necessary for the polar component to be further broken down in an electron-donor and an electron-acceptor component, e.g., $\delta_{i p} \propto \delta_{i p}^{+} \delta_{i p}^{-}$, and enter Eq. 2.60 as $\left(\delta_{1 p}^{+}-\delta_{2 p}^{+}\right)\left(\delta_{1 p}^{-}-\delta_{2 p}^{-}\right)$, rather than as $\left(\delta_{1 p}-\delta_{2 p}\right)^{2}$, otherwise one is led to rather unreasonable predictions (van Oss et al. 1988); thus, it becomes obvious that Eq. 2.60 is a simplification, which works well for polar substances 1 and 2 that are similar in polarity, but it becomes problematic when, for example, an electronegative and an electropositive substance are considered (Table 2.19).

While the tabulated data for the group contributions are given for amorphous materials at room temperature, $T=25{ }^{\circ} \mathrm{C}(298 \mathrm{~K})$, miscibility at processing temperatures $\left(200-300^{\circ} \mathrm{C}\right)$ is most often of interest; thus, it is necessary to correct the solubility parameter values for any temperature effects. The solubility parameters are, in principle, insensitive to temperature. However, although interaction energies are not expected to be a function of $T$, the corresponding interaction volumes, either for the polymers or for the corresponding groups, are indeed $T$ dependent. To account for the $T$ dependence, either Eq. 2.20 or 2.56 can be used. The calculated values of $\delta_{i d}, \delta_{i p}$, and $\delta_{i h}$ at $150{ }^{\circ} \mathrm{C}(423 \mathrm{~K})$ for selected polymers are listed in Table 2.20.

Equation 2.60 was also used to calculate the interfacial (interphasial) tension coefficients, $\gamma_{12}$, for two polymers forming an immiscible blend, based on their chemical structures.

$$
\begin{aligned}
\gamma_{12} & =k_{1}(\rho R T)^{n-1}\left[\Theta\left(\delta_{1 d}-\delta_{2 d}\right)^{2}+\left(\delta_{1 p}-\delta_{2 p}\right)^{2}+\left(\delta_{1 h}-\delta_{2 h}\right)^{2}\right]^{n} \\
& =k\left[\Theta\left(\delta_{1 d}-\delta_{2 d}\right)^{2}+\left(\delta_{1 p}-\delta_{2 p}\right)^{2}+\left(\delta_{1 h}-\delta_{2 h}\right)^{2}\right]
\end{aligned}
$$

where $k, k_{1}$ are constants and $\rho$ is the density. Good agreement was found between the computed and experimental values of the coefficient for 46 polymer blends (Fig. 2.16). The best correlation was found for the values $0.3 \leq \Theta \leq 0.5$. Thus, contrary to the dissolution processes dominated by the dispersive forces $(\Theta=4 \sim 5)$, for interphasial phenomena, the dispersive forces seem less important than the polar ones (Luciani et al. 1996a, 1997). A more detailed experimental approach, providing also $M_{w}$ and $T$ dependencies of the interfacial 
Table 2.19 Hansen solubility parameters for selected polymers at $25{ }^{\circ} \mathrm{C}$. All values are in $(\mathrm{J} / \mathrm{mL})^{2}$. For some polymers, more than one set of $\delta$ values are provided (a, b, etc.), indicating variability in experiments/fittings or polymer structure effects (comonomer, $M_{w}$, additives, etc.). A much more comprehensive collection of such values can be found in the relevant handbook (Hansen 2000)

\begin{tabular}{|c|c|c|c|c|}
\hline Polymer & & $\delta_{d}$ & $\delta_{p}$ & $\delta_{h}$ \\
\hline $\mathrm{ABS}$ & (a) & 18.60 & 8.80 & 4.20 \\
\hline ABS & (b) & 16.30 & 2.70 & 7.10 \\
\hline $\mathrm{ABS}$ & (c) & 17.60 & 8.60 & 6.40 \\
\hline $\mathrm{CA}$ & & 18.60 & 12.73 & 11.01 \\
\hline $\mathrm{CR}$ & & 19.00 & 8.00 & 0 \\
\hline HDPE & & 18.01 & 0 & 0 \\
\hline LLDPE & & 17.35 & 0 & 0 \\
\hline PA-6 & (a) & 17.39 & 12.71 & 11.14 \\
\hline PA-6 & (b) & 17.00 & 3.40 & 10.60 \\
\hline PA-66 & (a) & 18.62 & 5.11 & 12.28 \\
\hline PA-66 & (b) & 17.40 & 9.80 & 14.60 \\
\hline PAN & & 21.70 & 14.10 & 9.10 \\
\hline $\mathrm{PB}$ & & 16.98 & 0 & 1.02 \\
\hline $\mathrm{PC}$ & (a) & 19.10 & 10.90 & 5.10 \\
\hline $\mathrm{PC}$ & (b) & 18.10 & 5.90 & 6.90 \\
\hline PIB & & 14.53 & 2.52 & 4.66 \\
\hline PDMS & & 16.60 & 1.90 & 8.00 \\
\hline PES & & 19.60 & 10.80 & 9.20 \\
\hline PET & (a) & 19.44 & 3.48 & 8.59 \\
\hline PET & (b) & 19.10 & 6.30 & 9.10 \\
\hline PET & (Mylar) & 18.00 & 6.20 & 6.20 \\
\hline PMA & & 15.22 & 11.54 & 7.63 \\
\hline PnBA & & 16.38 & 8.97 & 5.77 \\
\hline PMMA & & 18.64 & 10.52 & 7.51 \\
\hline $\mathrm{PP}$ & & 17.19 & 0 & 0 \\
\hline PPS & & 18.70 & 5.30 & 3.70 \\
\hline PS & & 21.28 & 5.75 & 4.30 \\
\hline PSF & & 19.03 & 0 & 6.96 \\
\hline PTFE & & 16.20 & 1.80 & 3.40 \\
\hline PVAc & & 20.93 & 11.27 & 9.66 \\
\hline $\mathrm{PVC}$ & & 18.82 & 10.03 & 3.07 \\
\hline PVDF & & 9.65 & 5.87 & 6.66 \\
\hline PVP & & 21.40 & 11.60 & 21.60 \\
\hline SBR & & 17.55 & 3.36 & 2.70 \\
\hline
\end{tabular}

tension in immiscible blends, scattering or pendant drop approaches can be applied (Anastasiadis et al. 1988); this work showed good agreement with interfacial tension theoretical approaches, based on lattice models in the spirit of the Flory-Huggins approach (Helfand 1975a, b, c, Roe 1975). For more details 
Table 2.20 Calculated partial solubilities at $298 \mathrm{~K}$ and at $423 \mathrm{~K}$ (Luciani et al. 1996b)

\begin{tabular}{lllllllll}
\hline Polymer & $T^{*}(\mathrm{~K})$ & $V_{298} / V_{423}$ & $\delta_{d}^{298 K}$ & $\delta_{p}^{298 K}$ & $\delta_{h}^{298 K}$ & $\delta_{d}^{423 K}$ & $\delta_{p}^{423 K}$ & $\delta_{h}^{423 K}$ \\
\hline HDPE & 11,560 & 0.934 & 18.01 & 0 & 0 & 15.54 & 0 & 0 \\
\hline LLDPE & 9,710 & 0.915 & 17.35 & 0 & 0 & 14.32 & 0 & 0 \\
\hline PVAc & 9,389 & 0.911 & 15.22 & 11.54 & 7.63 & 12.44 & 9.43 & 6.23 \\
\hline PMA & 10,360 & 0.923 & 15.22 & 11.54 & 7.63 & 12.78 & 9.69 & 6.41 \\
\hline PS & 12,680 & 0.942 & 16.72 & 8.25 & 5.15 & 14.71 & 7.26 & 4.53 \\
\hline PEA & 9,929 & 0.918 & 15.75 & 10.45 & 6.83 & 13.08 & 8.68 & 5.67 \\
\hline PnBA & 8,590 & 0.899 & 16.38 & 8.97 & 5.78 & 13.00 & 7.12 & 4.59 \\
\hline PiBA & 8,590 & 0.899 & 16.12 & 8.99 & 4.61 & 12.80 & 7.14 & 3.66 \\
\hline PtBA & 8,590 & 0.899 & 14.37 & 8.16 & 8.34 & 11.41 & 6.48 & 6.62 \\
\hline PMMA & 11,880 & 0.937 & 13.59 & 9.25 & 10.30 & 11.79 & 8.03 & 8.94 \\
\hline PEHA & 8,160 & 0.891 & 16.81 & 7.27 & 3.69 & 13.10 & 5.67 & 2.87 \\
\hline PCP & 10,752 & 0.927 & 15.95 & 10.52 & 6.98 & 13.53 & 8.92 & 5.92 \\
\hline PET & 11,740 & 0.936 & 15.03 & 13.13 & 10.54 & 13.01 & 11.36 & 9.12 \\
\hline PA-6 & 15,290 & 0.956 & 17.39 & 12.71 & 11.14 & 15.78 & 11.53 & 10.11 \\
\hline PA-66 & 11,980 & 0.937 & 17.39 & 12.71 & 11.14 & 15.12 & 11.05 & 9.69 \\
\hline PEG & 10,170 & 0.921 & 16.74 & 10.14 & 8.74 & 14.00 & 8.48 & 7.31 \\
\hline PTMG & 10,300 & 0.922 & 17.33 & 7.51 & 6.24 & 14.53 & 6.30 & 5.23 \\
\hline PP & 11,260 & 0.932 & 16.70 & 0 & 0 & 14.32 & 0 & 0 \\
\hline PnBMA & 10,080 & 0.920 & 15.00 & 7.75 & 8.52 & 12.51 & 6.46 & 7.10 \\
\hline PtBMA & 13,340 & 0.946 & 13.45 & 7.17 & 9.58 & 11.94 & 6.36 & 8.50 \\
\hline PVDF & 10,440 & 0.924 & 9.65 & 5.87 & 6.66 & 8.13 & 4.94 & 5.61 \\
\hline PDMS* & 7,825 & 0.885 & 16.60 & 1.90 & 8.00 & 12.73 & 1.46 & 6.14 \\
\hline
\end{tabular}

Fig. 2.16 Interfacial tension coefficient at $150{ }^{\circ} \mathrm{C}$ for 46 polymer blends plotted versus the solubility parameter contributions. $R$ is the correlation coefficient

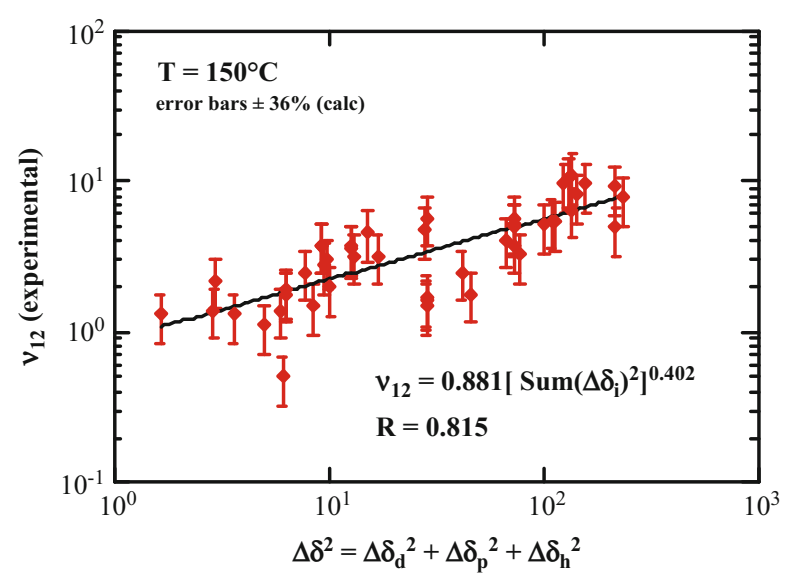

on the thermodynamics of interfacial tension in polymer blends, see Anastasiadis (2011).

A pragmatic modification of the solubility parameter approach was proposed by Bush et al. (1996). Recognizing that $\Delta H_{m}$ can be negative, the authors wrote 


$$
\Delta G_{m} \approx \Delta H_{m}=\phi_{1} \phi_{2}\left[\left(\delta_{1}-\delta_{2}\right)^{2}-e_{h}\right]
$$

where the correction $e_{h}$ is a positive number, associated with the energy density that originates from specific interactions. In other words, Eq. 2.62 implies that, in the presence of favorable specific interactions, in order to maximize miscibility the solubility parameters of the two blend components should be the same.

\subsubsection{Polymer Reference Interaction Site Model (PRISM)}

Thermodynamic properties of a system can also be obtained from atomistic considerations. Molecular dynamics or Monte Carlo methods have been successfully used to study polymers. The success stems from the fact that many properties can be projected from dynamics of relatively simple, oligomeric models. Unfortunately, miscibility strongly depends on the molecular weight, and so far it cannot be examined by these methods.

Another similar route that considers interactions between individual elements of a system is the reference interaction site model (RISM). The theory involves computations of the system structure by means of the probability density function, which describes location of all $N$ particles of the system. The binary interactions define the pair-density function:

$$
\rho_{N}^{(2)}=\frac{N !}{(N-2) !} \int P_{N} d r^{(N-2)}=\rho^{2} g(r)
$$

$P_{N}$ is the probability density of $N$ particles, $r$ is the particle coordinate, and $g(r)$ is the radial distribution function. The potential energy of the system is given as a product of the potential energy of a single particle with all others, multiplied by a factor $N / 2$ :

$$
U=\frac{N \rho}{2} \int_{0}^{\infty} 4 \pi r^{2} u(r) g(r) d r
$$

Thus, the total description of the thermodynamic state hinges on accurate quantification of $g(r)$. Ornstein and Zernike suggested separating the influence of this parameter into direct and indirect parts. The first describes direct interaction of the reference particle 1 with particle 2 , while the indirect one that of particle 1 with particle 3 , which in turn interacts with particle 2 :

$$
g_{12}(r)=c_{12}(r)+\rho \int c_{13}(r)\left[g_{13}(r)-1\right] d r_{3}
$$

where $c(r)$ is the direct correlation function. The advantage of the Ornstein-Zernike equation is that it can be readily generalized to more complex systems with inter- and 
intramolecular interactions. RISM has been applied to progressively more complex liquids, from monatomic to diatomic, $\mathrm{CCl}_{4}, \mathrm{C}_{6} \mathrm{H}_{6}$, etc. (Chandler and Andersen 1972). In the late 1980s, RISM has been applied to polymers, termed PRISM (Curro and Schweizer 1987; Schweizer and Curro 1989). The PRISM requires less computing time than atomistic simulations, but still makes it possible to incorporate structural details of polymeric molecules that the lattice models have been unable to account for. For example, bond lengths, their angles, chain conformation statistics, and different interaction potentials can be included in the mathematical simulations (Honeycutt 1992a, b, Curro 1994).

For polymer blends, PRISM provides good correlation with the experimental data obtained by SANS and light scattering (Eitouni and Balsara 2007). The agreement very much depends on selection of the "closure" approximation. Initial selection of the most appropriate closure for a given system can be quite difficult. Nevertheless, the method gave encouraging results, predicting details of phase separation in PO blends (Schweizer 1993). The deuteration effects as well as these related to structural differences between homopolymer and copolymers were well described (Schweizer 1993; Eitouni and Balsara 2007). The PRISM approach for polymer blends is still undergoing development and expansion; thus, more details here would simply be a snapshot at the moment of writing; the interested reader is therefore referred to seek a recent review or book chapter on PRISM.

\subsubsection{Summary of Theoretical Approaches}

Three theoretical approaches to the thermodynamics of polymer blends were briefly discussed: (1) the lattice theories, including the newer equation of state theories, (2) the off-lattice theories, and (3) the computational methods.

The lattice theories are the oldest and most frequently used to interpret and to predict the thermodynamic properties of multicomponent systems containing polymers. The Flory-Huggins theory is the best known. To use the theory, one must know the temperature, pressure, and concentration dependence of the enthalpic and entropic contributions to the binary interaction parameter, $\chi_{12}=\chi_{H}(T, P, \phi, \ldots)+$ $\chi_{s}(P, \phi, \ldots) / T$. Two types of extension of the FH theory were discussed, the first that evolved from Paul and Barlow is a heat of mixing approach, and the second was a solubility approach developed by Coleman and Painter. The first of these two makes it possible to treat a homopolymer as a copolymer composed of subunits of the chain, thus to generalize and predict behavior of a great number of polymer blends. The second divides all thermodynamic influences into three groups: the configurational entropy, the van der Waals interactions, and the specific interactions. The novelty of this modification is the method of treatment of the latter interactions that combines the FTIR-measured kinetics of associations with the FH theory. All these approaches suffer from the fundamental drawbacks of the FH theory: inability to take into account the fine structure of polymeric chains, nonrandomness, orientation, and free volume. The interaction parameters depend on many variables, and the reported numerical values vary widely. 
Fig. 2.17 Pressure dependence of the binodal and spinodal temperatures for the three $d$-PB/PS blends, with varied butadiene monomer structure. All phase boundaries increase with $P$, as expected from reduced free volume effects, but those for $d$-PB(1,2)/PS blends increase with a parabolic shape, while the increase is linear for the other two blends (Schwahn 2005)

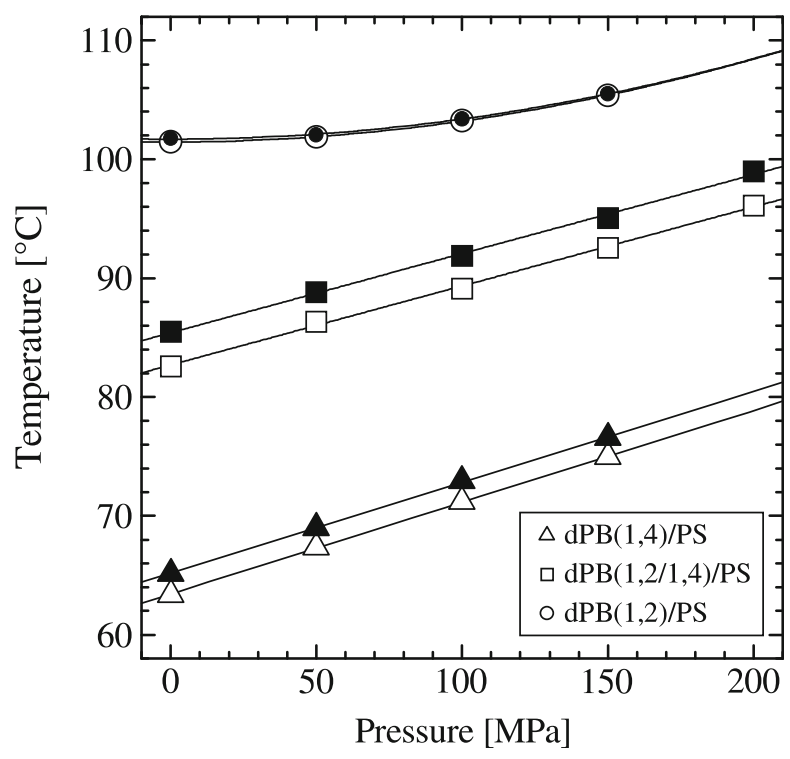

The theories based on the equation of state are more versatile. The model developed by Simha and many of his collaborators is most useful. By contrast with the FH theory, it leads to two binary interaction parameters, one energetic, the other volumetric, that are constant in the full range of independent variables. Furthermore, it has been found that the numerical values of these two parameters can be approximated by the geometric and algebraic averages, respectively. The nonrandom mixing can easily be incorporated into the theory. The non-lattice approaches, viz., strong interactions, heat of mixing, and solubility parameter approaches have more limited use. Their application should be confined to cases where the assumptions used in the derivations are well fulfilled.

The influence of pressure, $P$, on the miscibility needs a comment. Since pressure reduces the effects of the free volume contributions, for most blends the miscibility increase with $P$ (Walsh and Zoller 1987; Schwahn 2005). The effects are very sensitive to the monomer structure, as one would expect from free volume considerations, as, for example, in PB/PS blends (Fig. 2.17): In the case of $d$-PB/PS blends, the general trend of an increase of the phase boundaries with pressure is observed for all systems (viz., increased binodal and spinodal temperatures with $P$, due to the reduction of free volume), but the shapes of $T_{\text {binodal }}(P)$ and $T_{s}(P)$ are linear for $d$ - $\mathrm{PB}(1,4) / \mathrm{PS}$ and $d-\mathrm{PB}(1,4-c o-1,2) / \mathrm{PS}$ blends and are more parabolic for the blend with $d$-PB $(1,2) / \mathrm{PS}$; also the compatibility of PS is best for $d$ - $\mathrm{PB}(1,4)$ and worst for $d$ - $\mathrm{PB}(1,2)$, with the $d-\mathrm{PB}(1,4-c o-1,2)$ copolymer being in between the two, as expected (Fig. 2.17). The $P$ effect generally depends on the magnitude of the heat of mixing: For systems with $\Delta H_{m}<0$, the miscibility is enhanced by compression, whereas for those with $\Delta H_{m}>0$ it is reduced (Rostami and Walsh, 1984, 1985; Walsh and Rostami 1985). For PS solutions, the pressure gradient of 
the critical solution temperature was found to be a function of the molecular weight - the higher the $M_{w}$, the more negative the gradient value (Stroeks and Nies 1990). For polymer blends, the gradient is usually positive: for PPE blends with a random copolymer of $o$ - and $p$-fluorostyrene, the gradient $d(U C S T) /$ $d P=64$ to $108{ }^{\circ} \mathrm{C} / \mathrm{GPa}$ (Maeda et al. 1986), and for PS/PVME, $d(L C S T) /$ $d P=300{ }^{\circ} \mathrm{C} / \mathrm{GPa}$ (Hiramatsu et al. 1983), whereas for blends of PEA/PVF the LCST showed a complex dependence (Suzuki et al. 1982). The prediction of the pressure effects on solubility poses great challenges for the precision of the theoretical description. It should be noted that, according to the conditions for phase separation (vide infra, e.g., Eq. 2.66, Fig. 2.20), the critical point is given by third partial derivative of the free energy of mixing, and its pressure gradient - the stability condition - by the forth partial derivative.

\subsection{Phase Separation}

\subsubsection{Thermodynamics of Phase Separation}

The thermodynamic conditions for phase separation and, also, the definitions of the respective phase diagram are given by

$$
\begin{aligned}
& \text { binodal : }\left(\partial \Delta G_{m} / \partial \phi_{i}\right)_{P, T, n_{j}}^{\text {phase } 1}=\left(\partial \Delta G_{m} / \partial \phi_{i}\right)_{P, T, n_{j}}^{\text {phase } 2}\left(c f . \mu_{i}^{\text {phase } 1}=\mu_{i}^{\text {phase } 2}\right) \\
& \text { spinodal : }\left(\partial^{2} \Delta G_{m} / \partial \phi_{2}^{2}\right)_{P, T}=0 \\
& \text { critical point : }\left(\partial^{3} \Delta G_{m} / \partial \phi_{2}^{3}\right)_{P, T}=0 \\
& \text { stability condition : }\left(\partial^{4} \Delta G_{m} / \partial \phi_{2}^{4}\right)_{P, T}>0
\end{aligned}
$$

Schematic of a phase diagram of a binary system is shown in Fig. 2.18. There are three regions of different degree of miscibility: (1) the single-phase miscible region between the two binodals, (2) the four fragmented metastable regions between binodals and spinodals, and (3) the two-phase separated "spinodal" regions of immiscibility bordered by the spinodals. The diagram also shows two critical solution temperatures, the lower, LCST (at higher temperature), and the upper, UCST (at lower temperature). The phase diagram with two critical points is a rule for measurements of mixtures with low molecular weight component(s). Whereas for polymer blends usually only one critical point is accessible for normal conditions, e.g., $P$, and typically shows either the LCST (most often) or the UCST. A few blends having UCST are PS blends with SBS, PoClS, PBrS, or poly(methyl-phenyl siloxane), and BR blends with SBR, SAN with NBR (Utracki 1989).

The origin of the critical point can be traced to the temperature effects on miscibility: In a first approach, one can distinguish three principal contributions to the binary interaction parameter, $\chi_{12}$ (Patterson 1982), with rather distinct $T$ dependences: in general, the dispersive forces contributions in $\chi_{12}$, with a $1 / T$ 
Fig. 2.18 A schematic of the phase diagram for liquid mixtures with the upper and lower critical solution temperature, UCST and LCST, respectively. The placement of the critical compositions at about $\phi_{c r}=0.5$ denotes that this is a symmetric blend $\left(N_{1}=N_{2}\right)$. In the general case, the phase diagram is qualitatively the same, but much less symmetric with respect to $\phi$, with a $\phi_{c r}$ appearing in very small polymer concentrations, e.g., $\phi_{c r} \approx 1 / \sqrt{N}$, cf. FH theory

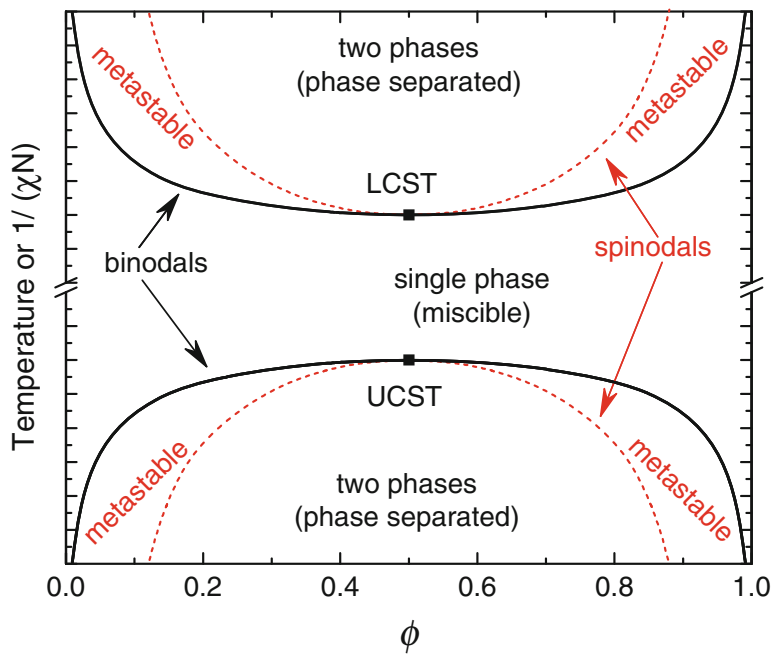

dependence; the free volume contributions, increasing with $T$; and the specific interaction contributions in $\chi_{12}$, typically increasing with $T$ (e.g., Arrhenius). As schematically illustrated in Fig. 2.19, the different temperature dependencies of these contributions affect $\chi_{12}$ in very different manner. For low molecular weight systems, where the dispersion interactions and free volume effects dominate the $\chi_{12}$, the sum of these two has a U-shape, intersecting the critical value of the binary interaction parameter in two places - hence, two critical points, UCST and LCST (Fig. 2.19a). In contrast, most polymer blends owe their miscibility to the presence of specific interactions that contribute a negative value in the interaction parameter, but increase with $T$ until they are balanced out by the free volume contributions (as well as by the ubiquitous dispersion forces, which in this case can be ignored). The sum of the two most important contributions in $\chi_{12}$ reaches the critical value at one temperature (e.g., an LCST, Fig. 2.19b).

To predict the phase behavior for a given system, the following steps are typically taken:

1. Select the most appropriate theoretical model for the free energy of mixing, $\Delta G_{m}$.

2. Determine values of the characteristic material parameters required by the selected theory.

3. Solve Eq. 2.66 for the selected theory.

4. Readjust the fitting parameters of the theory to optimize the fit.

5. Make predictions of the thermodynamic behavior and then verify experimentally.

The most important step is the selection of the theoretical model, i.e., the form of $\Delta G_{m}$. The balance between the complexity of its form and the adequacy of the description of experimental behavior must be preserved, also its applicability to the relevant $P$ and $T$ ranges must be checked, e.g., the existence of parameters with 
Fig. 2.19 Interactions in polymer solutions and blends usually comprise of dispersive forces, the free volume effects, and specific interactions. (a) The $\chi_{12}$ of polymer solutions are typically dominated by the contributions from dispersive forces and free volume, whose $T$ dependence can result in a UCST and LCST. (b) In polymer blends, the contributions from the free volume and the contributions from specific interactions usually control the $T$ dependence of $\chi_{12}$, giving rise to an LCST
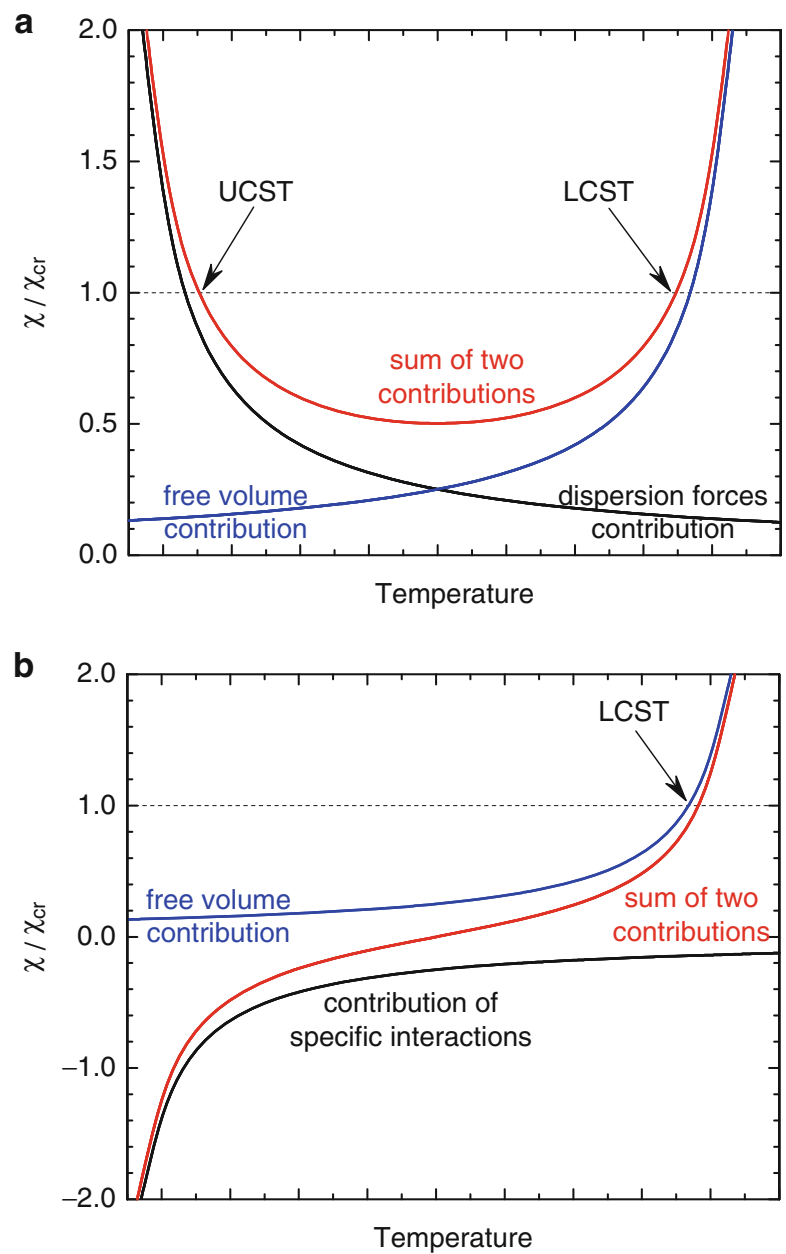

values of confidence in these ranges. One fundamental question is whether the pressure dependence of the phase diagram is important or not. Most data in literature deal with ambient pressures, whereas most industrial applications, viz., processing, compounding, and forming, are done at high pressures (e.g., extrusion $P \sim 70 \mathrm{MPa}$, injection molding $P \sim 500 \mathrm{MPa}$, etc.). The accuracy demanded from the theory to account for large variations of pressure is severe. It suffices to note that according to Eq. 2.66 the critical point is given by the third derivative of the free energy of mixing - its pressure dependence (stability criterion) is given by the fourth derivative!

The sometimes complicated forms of the free energy equations require, in most cases, that Eq. 2.66 are solved numerically, especially for the binodal determination (Jain and Simha 1984; Nies et al. 1990; Kisselev and Manias 2007). Often authors described the computational procedures, e.g., Nies et al. (1990), for the modified 
Fig. 2.20 Calculated phase diagrams for $n$-hexane/PE systems at varied pressures $(P=1,50$, and 100 bar $) ; x_{2}$ is the mole fraction of PE with $M_{w}=8 \mathrm{~kg} / \mathrm{mol}(\mathrm{Nies}$ et al. 1990)

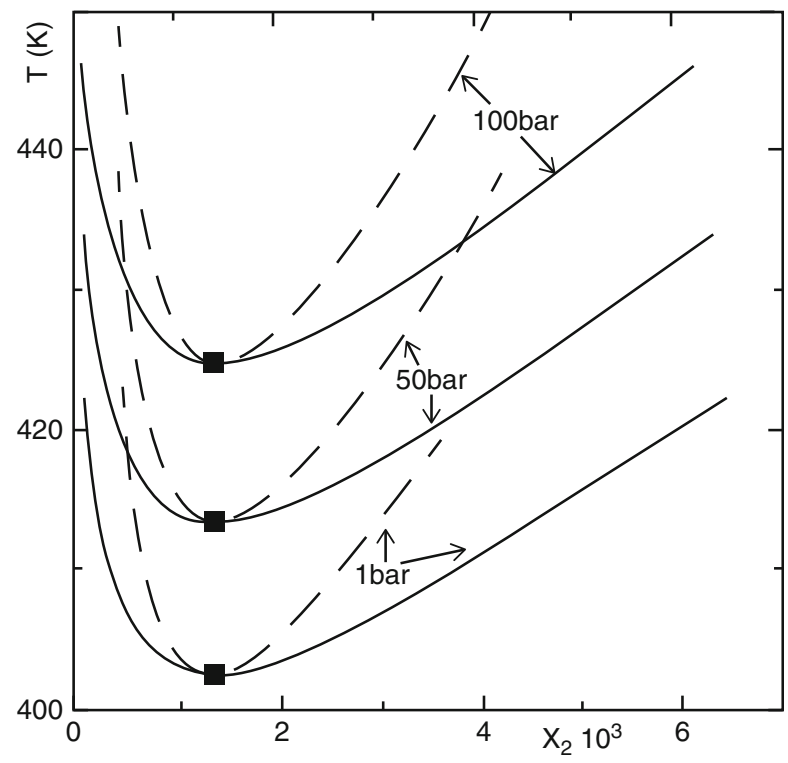

S-S theory giving Eqs. 2.42 and 2.43, and Kisselev and Manias (2007), for the LF theory with specific interactions. In the case of the S-S theory, the Helmholtz free energy is explicitly provided, which, in turn, can easily be converted into Gibbs free energy, viz., $G=F+P V$, or $\Delta G_{m}=\Delta F_{m}+P \Delta V_{m}$, where the terms with $\Delta$ represent the excess values due to mixing (differences from the single-phase values for each component, i.e., the function values of the mixture less the contributions of the pure components). Results of these computations are exemplified by the three isobaric phase diagrams of PE solutions in $n$-hexane, shown in Fig. 2.14, whereas a number of examples of polymer phase diagrams are available in literature (e.g., Koningsveld et al. 2001) (Fig. 2.20).

\subsubsection{Mechanisms of Phase Separation}

Phase separation takes place when a single-phase system undergoes either a change of composition or, more usually, a change in $T$ or $P$ that forces it to move from the single-phase region and enter either the metastable or the two-phase/spinodal region (Fig. 2.18). There is a substantial difference between the phase separation mechanisms that take place for moving from single phase to metastable and for moving from single phase to two phase. When the system enters from the singlephase region into the metastable region, phase separation typically occurs by mechanisms resembling crystallization, i.e., slow nucleation followed by growth of the phase separated domains. Thus, this process is known as the "nucleation and growth," or NG for short. In contrast, when the system is forced to jump from a single phase into the spinodal/two-phase immiscible region, the phases separate 


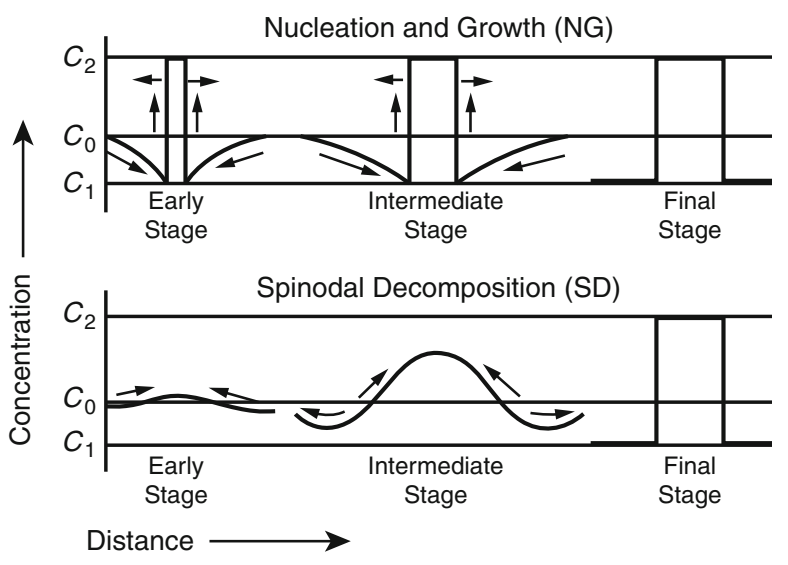

Fig. 2.21 Schematic representation of the density fluctuations during the spinodal decomposition mechanism $(S D$, bottom) and the nucleation and growth $(N G, t o p)$. Three stages are shown: early, where in SD the wavelength is constant but the amplitude increases; intermediate, where both the wavelength and the amplitude change; and final, where the concentration amplitude is at maximum and the wavelength increases only due to coarsening processes

spontaneously: This process starts with instantaneous segmental density fluctuation that progressively increases in amplitude and later in wavelength. The process is known as the "spinodal decomposition," or SD for short.

These two processes are schematically illustrated in Fig. 2.21, as composition versus distance. Three stages of the phase separation process are distinguished: early, intermediate, and late/final. The upper and lower limits of the concentration are given by the tie-line limits intersecting the binodal at $T=$ constant, for the temperature of the experiment (Fig. 2.18). Following the late stage of phase separation, the process continues into a series of coalescence steps, which increase the lateral dimension $d$ of the phases: Coalescence starts with Ostwald ripening ( $d \propto t^{1 / 3}$; see Eq. 2.67), followed by surface tension-controlled coarsening $(d \propto t$; see Eq. 2.68), ending with gravitational or divergent coalescence that leads to formation of large size (macroscopic) separate phases.

$$
d V_{d} \propto \frac{\gamma_{12} \phi_{c} V D_{t}}{R T} d t \Rightarrow\left(d / d_{o}\right)^{n_{c}}=1+K_{c} t
$$

where $V_{d}$ is the drop volume, $\gamma_{12}$ is the interfacial tension, $\phi_{c}$ is the equilibrium concentration of the phase separated system (binodal concentration at $T$ ), $V$ is the molar volume of the drop fluid, $n_{c}$ is the coarsening exponent, and $K_{c}$ is the coarsening rate constant. For a steady-state coarsening, $n_{c}=3$, whereas for sheared systems $n_{c}=3 / 2$ (Ratke and Thieringer 1985). Subsequently,

$$
\frac{R T}{\gamma_{12}} \leq d^{2} \leq \frac{\gamma_{12}}{g \Delta \rho} \Rightarrow d \propto t
$$


where $g$ is the gravitational constant (acceleration) and $\Delta \rho$ is the density difference between the two phases.

For fundamental studies of phase separation, to ensure that phase separation will proceed by the SD mechanism, a composition near the critical point is usually selected. The blend is then quenched (temperature jump) from the miscible to the spinodal region passing through or near the critical point. For this reason, SD studies are often called "critical quenching." Conversely, to study the NG mechanism (a significantly less popular subject), compositions for temperature jumps are selected away from the critical concentration; these studies are then known as "off critical." Reviews on phase separation are continuously published (the reader is strongly encouraged to do a fresh literature search); some such reviews were used next to highlight phase separation behaviors (Kwei and Wang 1978; Olabisi et al. 1979; Herkt-Maetzky 1984; Aifantis 1986, 1987; Hashimoto 1987; Nose 1987; Binder 1987; Hashimoto 1988; Han et al. 1988; Utracki 1989, 1994; etc.).

\subsubsection{Spinodal Decomposition (SD)}

Early theories of the phase separation dynamics are based on a mean-field approach developed for metallurgical applications (Cahn and Hilliard 1958; Cahn 1978). In the spinodal region, the concentration fluctuations are delocalized, leading to long-range spontaneous phase separation by SD. This type of mean-field theory is not adequate to describe the phase dynamics of small molecule liquids (especially near the critical point), but it has been successful in describing phase separation in polymeric systems, due to the slow diffusion rates owing to the large-chain dimension. The time-dependent probability distribution function for concentration can be determined directly by digital image analysis method (Tanaka and Nishi 1987).

For SD, three stages and three mechanisms of domain growth are traditionally identified (Siggia, 1979): diffusion, liquid flow, and coalescence. The earliest diffusion stage follows the Ostwald equation, Eq. 2.67, and is limited to the period when $d_{o} \leq d \leq 5 d_{o}$, where $d_{o}$ is the initial diameter of the segregated region $\left(d_{o} \approx\right.$ 2-9 nm Voigt-Martin et al. 1986). The subsequent flow region dominates when $5 d_{o} \leq d \approx 1 \mu \mathrm{m}$. Within these two regions, the SD structure is regular and the growth can be observed by scattering methods. At the last, coalescence, stage of SD, diffusion becomes bimodal and then irregular. Thus, at this stage, the kinetics of phase separation has been studied using time-resolved scattering techniques, with light, neutrons, or other irradiation sources. There is a direct relation between the virtual structure function, $S(q, t)$, and the scattering intensity function, $I(q, t)$ :

$$
\begin{aligned}
I(q, t) & =I_{b}+K S^{2}(q, t) \\
& =\left(I_{b}+K S_{\infty}\right)+K\left(S_{o}-S_{\infty}\right) \exp [2 R(q) t] \\
& =I_{\infty}+\left(I_{o}+I_{\infty}\right) \exp [2 R(q) t]
\end{aligned}
$$

where $I_{b}$ is the background scattering intensity, $K$ is a constant, and $S_{o}$ and $S$ are the values of the structure function at time 0 and at time $t$. To extract the concentration 
fluctuation function $R(q)$, a semilogarithmic plot of $\ln \left(I-I_{\infty}\right)$ versus $t$ is used. However, in many cases, since the scattering intensity at equilibrium is low, the concentration fluctuation function is determined as

$$
R(q) \cong \frac{1}{2} \frac{d \ln I(q, t) / I_{o}(q, t)}{d t}
$$

As Eq. 2.69 indicates, the scattering intensity $I(q, t)$ is proportional to $S^{2}(q, t)$. For this reason, the plot of $I(q, t)$ versus $q$ (at constant decomposition time and temperature) already provides evidence of the dynamics of phase separation in polymer blends.

The mechanism of phase separation is analyzed from the $R$ versus $q$ dependence. The dynamics of phase separation within the SD domain starts with a balance between the thermodynamics and material flux. The mean-field theory of phase separation leads to the following simple form of the structure function, $S(q)$ (Cahn and Hilliard 1958):

$$
\begin{aligned}
& S(q)=S_{o} \exp [R(q) t] \\
& R(q)=-M q^{2}\left[\frac{\partial^{2} G}{\partial \phi^{2}}+2 q^{2} k\right] \\
& q=\frac{4 \pi}{\lambda} \sin (\Theta / 2)=\frac{2 \pi}{\Lambda}
\end{aligned}
$$

where $M$ is the Onsager mobility factor, $G$ is the free energy for the homogenous system, $\phi$ is the segmental volume fraction, and $\kappa$ is the gradient energy coefficient arising from local composition fluctuations; the wavevector, $q$, is a function of the wavelength, $\lambda$, and the scattering angle, $\Theta$. It has been shown that near the spinodal $\partial^{2} G / \partial \phi^{2} \propto 1-\left(T / T_{s}\right)$, where $T_{s}$ is the spinodal temperature, whereas the maximum wavelength for SD phase separation is $\lambda_{\max } \propto\left[1-\left(T / T_{s}\right)\right]^{-1 / 2}$ (van Aarsten 1970). According to Eq. 2.71, the concentration fluctuation function, $R(q)$, can be linearized by plotting $R(q) / q^{2}$ versus $q^{2}$. The linearity provides evidence of the SD mechanism independently of the scale of the phase separation.

From the intersection at $q=0$, the mutual diffusion coefficient $D_{M}$ is obtained:

$$
\begin{aligned}
& \lim _{q \rightarrow 0}\left(\frac{R(q}{q^{2}}\right)=D_{M}=-M \frac{\partial^{2} G}{\partial \phi^{2}} \\
& D_{M}=2 \phi_{1} \phi_{2}\left(\chi_{12 s}-\chi_{12}\right)\left(N_{1} D_{1} \phi_{2}+N_{2} D_{2} \phi_{1}\right) \\
& \chi_{12 s}=\left(N_{1} \phi_{1}\right)^{-1}+\left(N_{2} \phi_{2}\right)^{-1}
\end{aligned}
$$

Evidently, $D_{M}$ depends on the second derivative of the free energy, which in turn can be expressed in terms of the self-diffusion coefficients of polymers, $D_{1}$ and $D_{2}$, and the $\chi(T)$-distance from spinodal. The method of determining $D_{M}$ is presented in Fig. 2.22, whereas its dependence on $T$ across the spinodal is shown in Fig. 2.23. From $D_{M}$, one may calculate the binary interaction parameter and, 

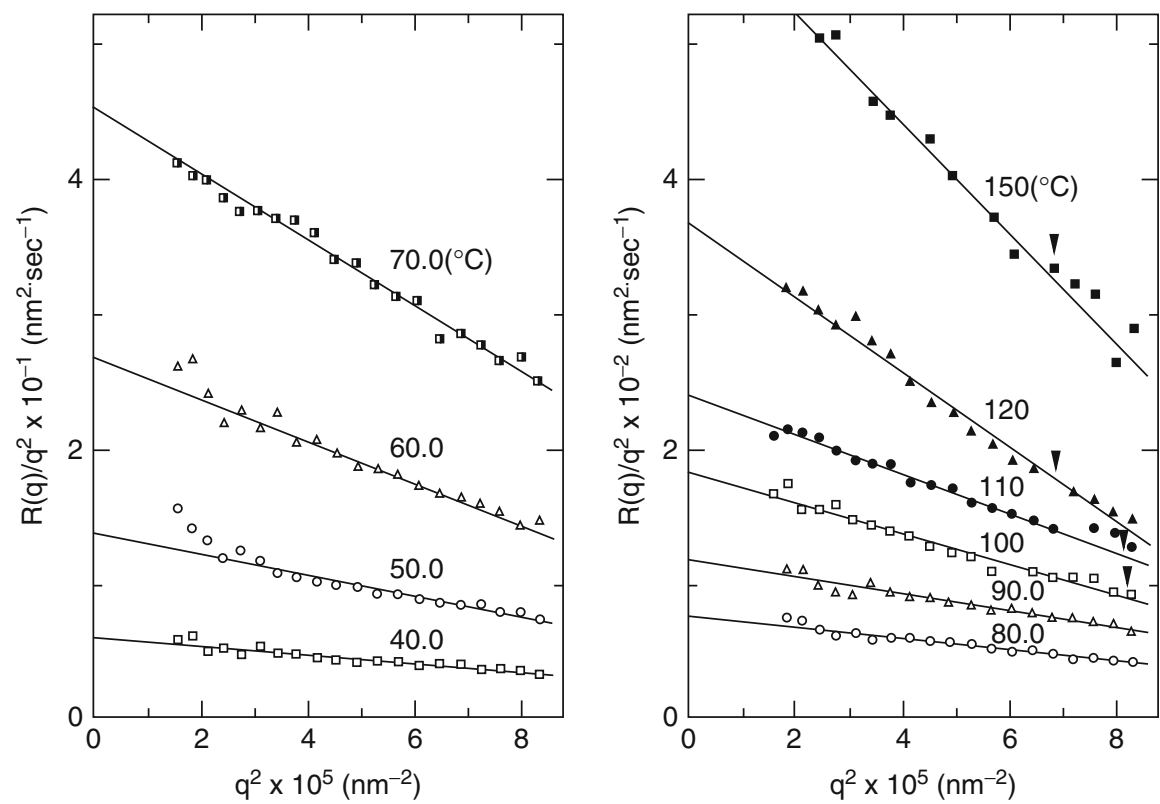

Fig. 2.22 Determination of the mutual diffusion coefficient, $D_{M}$, from the early stage data of the spinodal decomposition, SD, in SBS/PBD blends (Izumitani and Hashimoto 1985)

Fig. 2.23 Mutual diffusion coefficient $D_{M}$ versus $T$ for a PS/PVME blend undergoing spinodal decomposition. $T$-jumps, across the spinodal temperature $T_{s}=151.2^{\circ} \mathrm{C}$, were performed in both directions, quenching into SD region, and reverse, quenching from the $\mathrm{SD}$ region (Sato and Han 1988)

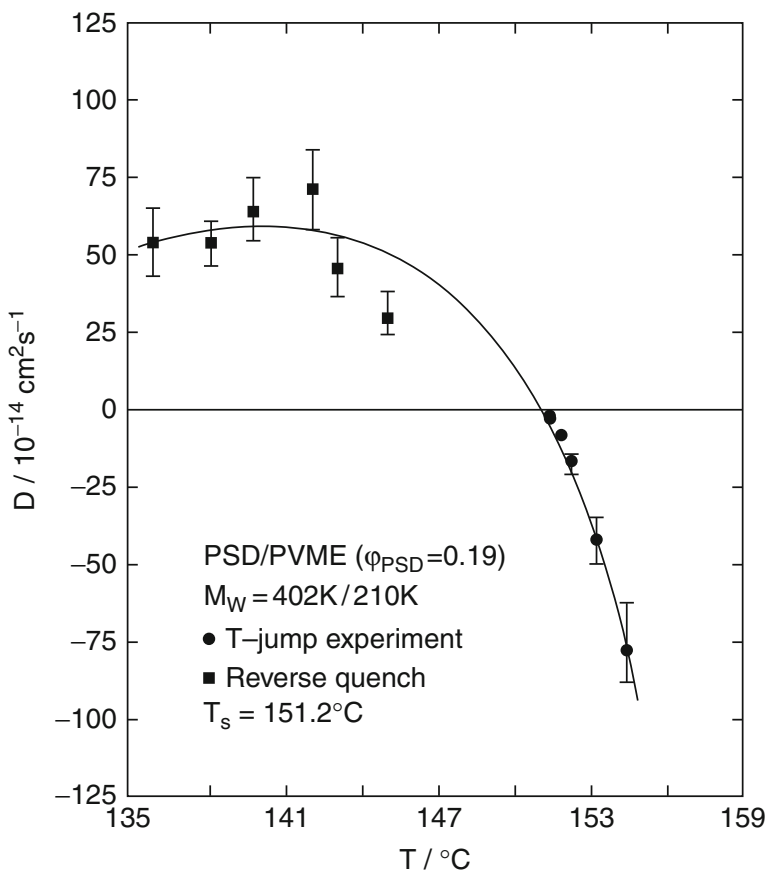


Fig. 2.24 Binary interaction parameters $\chi_{12}$ versus temperature in PVME/d-PS blends; scattering data from three different blend compositions $\phi$ are shown, the dashed lines correspond to the respective spinodal points at each composition $\phi$ (Herkt-Maetzky 1983)

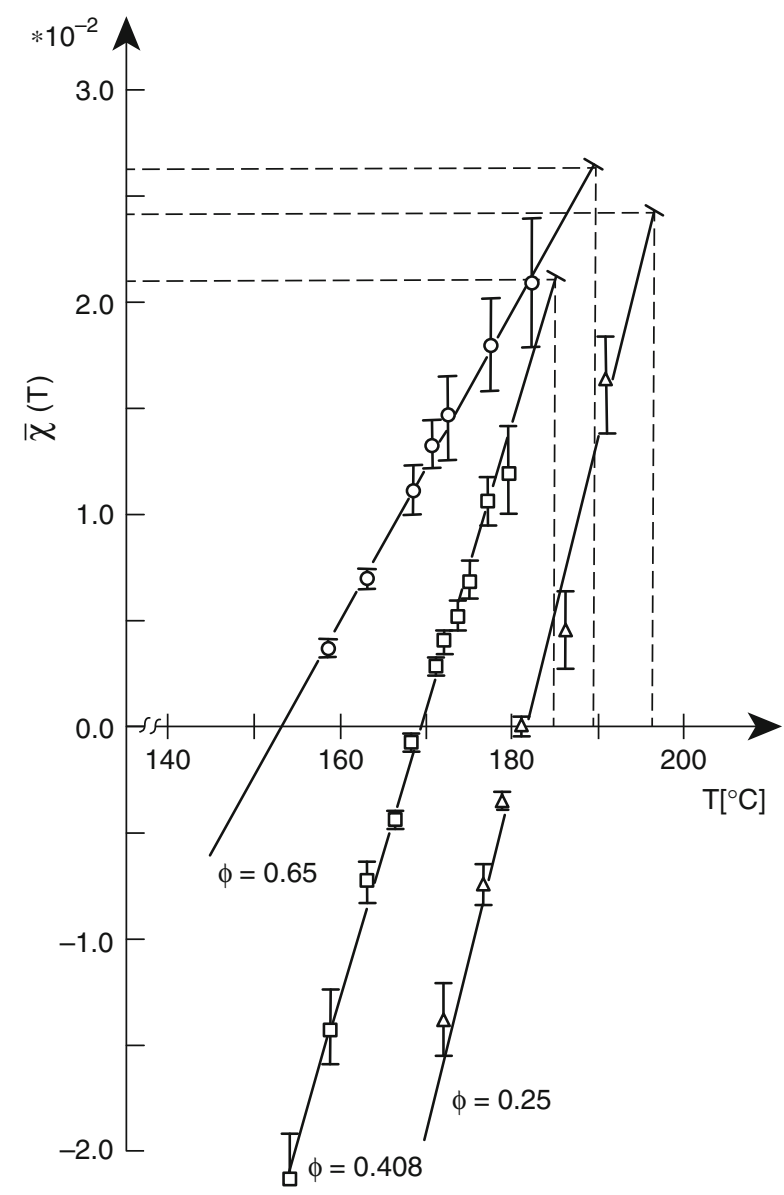

hence, determine conditions for the thermodynamic miscibility. Fig. 2.24 shows the $\chi_{12}$ dependence on $T$ and $\phi$.

Coarsening of the structure continues through a series of steps that lead to a gross (macroscopic) phase separation. However, under certain conditions, the coarsening progresses only up to a certain stage, where the structure becomes fixed or "pinned." The pinning originates in a transition from the percolation to cluster formation then freezing-in of the molecular diffusion. In simple term, the generated structure is relatively regular; thus, there is little energetic incentive for the molecules to diffuse from one drop, through "unfriendly" territory of the other phase, to another drop. An example of this has been given for blends of PI containing 20, 30, and $50 \mathrm{wt} \%$ of SBR (Takenaka et al. 1989). Evidently, stirring the mixture disrupts the fine balance of forces that make the pinning process possible; thus, pinning is not expected to take place during processing.

Most work on SD focuses on the effects of temperature and composition on phase equilibria in binary polymer mixtures. However, in industrial processes, other 
variables may be of equal importance, e.g., the shear stress, shear stress rate, and pressure. It is known that these variables are important for miscibility and, hence, for the morphology and performance. For example, during extrusion of $\mathrm{PC} / \mathrm{PBT}$ blends the LCST was increased by at least $60{ }^{\circ} \mathrm{C}$, causing miscibility; the blend, upon exit from the extruder, phase separated by the SD mechanism, which resulted in co-continuity of phases and excellent performance. Solvent casting of polymer blends and controlled evaporation can also lead to SD. This technology has been used for industrial production of semipermeable, selective membranes. The product characterized by co-continuity of phases also showed excellent mechanical performance. The type of solvent, concentration, temperature, and method of casting are used to control the blend morphology and its final performance (Inoue et al. 1985, 1987; Nauman et al. 1986).

Phase separation was computer simulated using finite-difference in time and space Runge-Kutta and Monte Carlo with a Hamiltonian methods (Petschek and Metiu 1983; Meakin and Reich 1982; Meakin et al. 1983). Both methods were found equivalent, reproducing the observed pattern of phase separation in both NG and SD regions. The unity of the phase separation dynamics on both sides of the spinodal has been emphasized (Leibler 1980; Yerukhimovich 1982).

\subsubsection{Nucleation and Growth (NG)}

As shown in Fig. 2.25, there is a significant difference in the scattering pattern evolution for NG and SD mechanisms, especially during the early stages of phase separation: SD follows a semilogarithmic time dependence (see Eq. 2.71), whereas NG follows a linear time dependence.

When the concentration of the minor phase is above 10-15\%, SD occurs by rapid growth of regularly spaced concentration waves, while NG is a slower and more random process. On the other hand, at low concentrations of the minor phase, neglecting the fine structure of the dispersed phase, phase separation by NG and SD mechanism looks similar. Also the reverse quenching of SD and NG morphologies is similar, both being controlled by (Kumaki and Hashimoto 1986):

$$
\left(\frac{\partial^{2} G}{\partial \phi^{2}}\right)_{\text {spinodal }}=-\lim _{q \rightarrow 0} \frac{R(q)}{M q^{2}}=\mathrm{constant}
$$

The relation shows that the rate depends on the conditions for SD, the same for systems on both sides of the spinodal.

The NG is an activated process with a linear growth rate (Matsuda 1991):

$$
\begin{array}{ll}
\text { Nucleation : } & \frac{d N}{d t}=k_{N} \exp \left[-\Delta E_{a N} / k_{B} T\right] \\
& \begin{array}{ll}
\Delta E_{a N}=\frac{4}{3} \pi r_{N, c r}^{3} \Delta G_{m}+4 \pi r_{N, c r}^{3} \gamma_{12}=\frac{16 \pi}{3} \gamma_{12}^{3} \Delta G_{m}^{2} \\
\text { Growth : } \quad & \frac{\partial \phi}{\partial t}=D \nabla^{2} \phi
\end{array}
\end{array}
$$


Fig. 2.25 A plot of the scattering intensity versus time for $70 \%$ PVME $/ 30 \%$ PS blends (SE-70), at wavevector $q=5.6 \times 10^{4} \mathrm{~cm}^{-1}$ (the spinodal temperature was estimated at $T_{s}=99.2^{\circ} \mathrm{C}$ ); the data at higher $T\left(99.7^{\circ} \mathrm{C}\right.$, $100{ }^{\circ} \mathrm{C}$, and $101^{\circ} \mathrm{C}$ ) are for the $\mathrm{SD}$ regime, and the data for $99{ }^{\circ} \mathrm{C}$ are for the ND regime (Hashimoto et al. 1983)

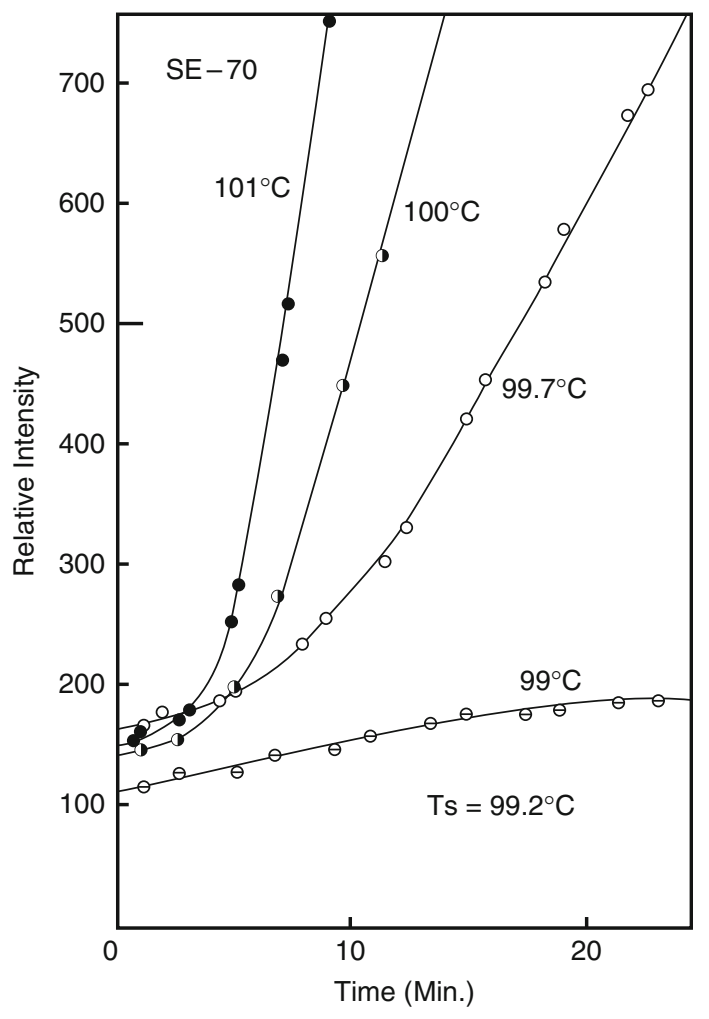

where $r_{N, c r}$ is the critical radius of the nucleating particle and $D$ is the diffusion constant. Accordingly, the nucleation process requires activation energy for nucleation $\left(\Delta E_{a N}\right)$. However, once nucleated, the phases separate and grow spontaneously. The nucleation is initiated by the local fluctuations of density. The activation energy $\Delta E_{a N}$ depends on the local gradient of the free energy of mixing $\left(\Delta G_{m}\right)$ and the interfacial tension $\left(\gamma_{12}\right)$. Once formed, the drops grow by diffusion of macromolecules into the nucleated domains, with the rate given by the Ostwald ripening. The diffusion stage is followed by coalescence (Hashimoto et al. 1986; Hashimoto 1988).

In principle, during the initial stage of phase separation the NG mechanism leads to a drop/matrix morphology over the full range of concentrations. However, the morphology at the later stages depends on the volume fraction of the dispersed phase, as well as the method and extent of stabilization. Furthermore, since nucleation depends on the local density fluctuations, whose amplitude depends on the distance from the critical conditions, near the spinodal, phase separation can occur either by the NG or SD mechanism (Langer 1977, 1980). This ambiguity is further exacerbated in applications by compounding and by processing stresses. 
Table 2.21 Phase equilibrium studies for polymer/solvent systems, including mixed solvents

\begin{tabular}{|c|c|}
\hline Polymer & Solvent \\
\hline $\mathrm{PE}$ & $\begin{array}{l}\text { Diphenyl-ether, } n \text {-hexane, ethylene, nitrobenzene, amyl acetate, xylene, } 1,2,4,5- \\
\text { tetrachlorobenzene (TCB), xylene/glycol, toluene, petroleum ether, bromonaphthalene }\end{array}$ \\
\hline PP & Benzene, $o$-dichlorobenzene, isopropyl ether, $n$-heptane, tetralin/butyl carbitol, TCB \\
\hline PDMS & Oligo-isobutylene, carbon tetrachloride/cyclohexane/methanol \\
\hline PS & $\begin{array}{l}\text { Cyclohexane, methyl-cyclohexane, acetone, tert-butyl acetate, ethyl acetate, ethyl } \\
\text { oxalate, vinyl acetate, ethyl malonate, methyl acetate, methyl succinate, octene, } \\
\text { polyvinyl acetal/chloroform, rubber/benzene, toluene/ethanol }\end{array}$ \\
\hline $\mathrm{PVC}$ & Tetrahydrofuran/water, cyclohexanone/methanol, chlorobenzene, cyclohexane/acetone \\
\hline PVP & Water/acetone \\
\hline PVAc & Acetone, water, methyl-isobutyl ketone, dioxane/isopropanol \\
\hline PVA1 & Water, water/acetone, water/propanol \\
\hline PMMA & $\begin{array}{l}\text { Benzene, toluene, } 3 \text {-heptanone, } n \text {-propanol, } p \text {-cymene, MEK/isopropanol, acetone/ } \\
\text { hexane, butanone/cyclohexane }\end{array}$ \\
\hline PBMA & Benzene \\
\hline PCHMA & Dioxane/methanol \\
\hline PEG & Water, chloroform/hexane \\
\hline PPG & Water, isopropanol/water, isooctane \\
\hline PIB & $\begin{array}{l}\text { Di-isobutyl ketone, benzene/acetone, tri-methyl pentene, 2-methylheptane, toluene/ } \\
\text { methanol }\end{array}$ \\
\hline $\mathrm{CR}$ & Benzene, benzene/acetone, benzene/methanol \\
\hline PIP & Acetone/ethanol \\
\hline PAN & Dimethyl formamide, dimethyl sulfoxide/toluene \\
\hline $\mathrm{PC}$ & Methylene chloride/methanol \\
\hline PET & Phenol/cyclohexane, dimethyl formamide, trifluoroacetic acid/chloroform \\
\hline PA-6 & $m$-Cresol/cyclohexane, phenol, formic acid \\
\hline $\mathrm{NC}$ & Acetone, butyl acetate, acetone/water, ethyl acetate/heptane \\
\hline SBR & Benzene \\
\hline
\end{tabular}

\subsubsection{Phase Diagrams}

There is a large body of literature on phase diagrams for binary and ternary polymer solutions (Flory 1953; Tompa 1956; Cantow 1967; Utracki 1989) and extensive compilations of data (phase diagrams, cloud points, critical temperatures) for numerous systems (e.g., Wohlfarth 2004, 2008; Koningsveld et al. 2001). A few examples of such systems are listed in Table 2.21.

Phase diagrams of polymer blends, including binary polymer/polymer systems and ternary polymer/polymer/solvent systems, are scarcer (Koningsveld 2001). Furthermore, owing to the recognized difficulties in determination of the equilibrium properties, the diagrams are oftentimes either partial or approximate or built using low molecular weight polymers. Examples are listed in Table 2.22. 
Table 2.22 Phase equilibrium studies for polymer/polymer blend systems. CST stands for critical solution temperature: $L$ indicates lower CST, while $U$ indicates upper CST (see Fig. 2.18)

\begin{tabular}{|c|c|c|c|c|c|}
\hline \multicolumn{2}{|l|}{ Polymer-1 } & \multicolumn{2}{|l|}{ Polymer-2 } & \multirow{2}{*}{$\begin{array}{l}\mathrm{CST}\left({ }^{\circ} \mathrm{C}\right) \\
L=120\end{array}$} & \multirow{2}{*}{$\begin{array}{l}\text { References } \\
1\end{array}$} \\
\hline PS & $\left(M_{w}=237\right)$ & PVME & $\left(M_{w}=13.3\right)$ & & \\
\hline PS & & TMPC & & $L<220$ & 2 \\
\hline PS & $\left(M_{w}=29\right)$ & PoClS & & $L=350$ & 3 \\
\hline PS & $\left(M_{w}=22\right)$ & $\mathrm{P}(\mathrm{S}-c o-\mathrm{BrS})$ & $\left(M_{w}=22\right)$ & $U=218$ & 4 \\
\hline PS & $\left(M_{w}=10\right)$ & PMPS & $\left(M_{w}=2.8\right)$ & $U=103$ & 5 \\
\hline PS & $\left(M_{w}=2.7\right)$ & PIP & $\left(M_{w}=2.7\right)$ & $U=173$ & 6 \\
\hline SAN & $\left(M_{w}=223\right)$ & PMMA & $\left(M_{w}=92\right)$ & $L=150$ & 7 \\
\hline SAN & $\left(M_{w}=223\right)$ & PCL & $\left(M_{w}=35\right)$ & $L=90$ & 8 \\
\hline SAN & $\left(M_{w}=194\right)$ & NBR & $\left(M_{w}=297\right)$ & $L=52$ & 9 \\
\hline $\mathrm{PVC}$ & $\left(M_{w}=55\right)$ & PMMA & $\left(M_{w}=60\right)$ & $L=190$ & 10 \\
\hline $\mathrm{PVC}$ & $\left(M_{w}=160\right)$ & NBR & $\left(M_{w}=340\right)$ & $L>180$ & 11 \\
\hline PVDF & $\left(M_{w}=100\right)$ & PEMA & $\left(M_{w}=332\right)$ & $L=200$ & 12 \\
\hline$\alpha \mathrm{MSAN}$ & $\left(M_{w}=160\right)$ & PMMA & $\left(M_{w}=126\right)$ & $L=185$ & 13 \\
\hline $\mathrm{CPE}$ & $\left(M_{w}=190\right)$ & PMMA & $\left(M_{w}=42\right)$ & $L=100$ & 14 \\
\hline PES & & PEG & $\left(M_{w}=20-200\right)$ & $L=80$ & 15 \\
\hline $\mathrm{BR}$ & $\left(M_{w}=390\right)$ & SBR & $\left(M_{w}=480\right)$ & $U=103$ & 16 \\
\hline PHMA & $\left(M_{n}=55\right)$ & StVPh & $\left(M_{n}=11\right)$ & $L=159$ & 17 \\
\hline PHMA & $\left(M_{n}=55\right)$ & StVPh & $\left(M_{n}=14\right)$ & $L=84$ & 17 \\
\hline PPE & & PFSt copolyr & & $U=270$ & 18 \\
\hline PVME & & $\mathrm{StVPh}(0-0$. & $-\mathrm{OH})$ & $L=153-193$ & 19 \\
\hline PEK & & N-TPI & & $U=445$ & 20 \\
\hline PC-co-TMPC & $\left(M_{w}=72\right)$ & PS & $\left(M_{w}=330\right)$ & $L=175$ & 21 \\
\hline PC-co-TMPC & $\left(M_{w}=72\right)$ & SAN & $\left(M_{w}=160\right)$ & $U=290$ & 21 \\
\hline PC-co-TMPC & $\left(M_{w}=72\right)$ & SMMA & $\left(M_{w}=160\right)$ & $U=230$ & 21 \\
\hline P- $n$-BMA & $\left(M_{w}=270\right)$ & PMMA & $\left(M_{w}=100\right)$ & $U=160$ & 22 \\
\hline SMAN & $\left(M_{w}=101\right)$ & PMMA & & $L=135$ & 23 \\
\hline PVME & $\left(M_{w}=99\right)$ & PS & $\left(M_{w}=114\right)$ & $L=119$ & 24 \\
\hline PVME & $\left(M_{w}=389\right)$ & PS & $\left(M_{w}=230\right)$ & $L=152$ & 25 \\
\hline PEG & $\left(M_{w}=300\right)$ & PMMA & $\left(M_{w}=130\right)$ & $L=227$ & 26 \\
\hline PI & $\left(M_{w}=101\right)$ & $d$-PB & $\left(M_{w}=53\right)$ & $L=85$ & 27 \\
\hline PVDC & $\left(M_{w}=101\right)$ & PHEDO & & $L=174$ & 28 \\
\hline PVDF & $\left(M_{w}=428\right)$ & PMA & $\left(M_{w}=257\right)$ & $L=332$ & 29 \\
\hline PVDF & $\left(M_{w}=736\right)$ & PMA & $\left(M_{w}=257\right)$ & $L=300$ & 29 \\
\hline PVDF & $\left(M_{w}=140\right)$ & PBA & $\left(M_{w}=14\right)$ & $L=233$ & 30 \\
\hline PIB & $\left(M_{w}=1,000\right)$ & LLDPE & $\left(M_{w}=125\right)$ & $L=85$ & 31 \\
\hline $\mathrm{PC}$ & $\left(M_{w}=58\right)$ & PMMA & $\left(M_{w}=87\right)$ & $L=240$ & 32 \\
\hline PVC & $\left(M_{w}=170\right)$ & PPrA & $\left(M_{w}=87\right)$ & $L=129$ & 33 \\
\hline $\mathrm{PVC}$ & $\left(M_{w}=170\right)$ & PPeA & $\left(M_{w}=415\right)$ & $L=107$ & 33 \\
\hline PVC & $\left(M_{w}=170\right)$ & PBA & $\left(M_{w}=383\right)$ & $L=126$ & 33 \\
\hline TMPC & $\left(M_{w}=33\right)$ & SMMA & $\left(M_{w}=281\right)$ & $L=250$ & 34 \\
\hline
\end{tabular}


Table 2.22 (continued)

\begin{tabular}{llllll}
\hline Polymer-1 & \multicolumn{3}{c}{ Polymer-2 } & CST $\left({ }^{\circ} \mathrm{C}\right)$ & References \\
\hline TMPC & $\left(M_{w}=33\right)$ & SMMA & $\left(M_{w}=106\right)$ & $L=264$ & 34 \\
\hline PMMA & $\left(M_{w}=94\right)$ & SMMA & $\left(M_{w}=81\right)$ & $L=150$ & 35 \\
GMA/MMA & $\left(M_{w}=471\right)$ & SAN & $\left(M_{w}=152\right)$ & $U=350$ & 36 \\
PPE & $\left(M_{w}=39\right)$ & P $\alpha$ MS & $\left(M_{w}=55\right)$ & $L=253$ & 37 \\
\hline PVC & $\left(M_{w}=213\right)$ & $\alpha$ MSAN & $\left(M_{w}=55\right)$ & $U=261$ & 37 \\
PVC & $\left(M_{w}=213\right)$ & SAN $(20 \% \mathrm{AN})$ & $\left(M_{w}=178\right)$ & $U=150$ & 37 \\
\hline
\end{tabular}

Polymer abbreviations: $P H M A$ poly- $n$-hexyl methacrylate, $S t V P h$ polystyrene-co-vinylphenol, PFSt poly(o-fluorostyrene-co-p-fluorostyrene), $\mathrm{P}(\mathrm{S}$-co- $\mathrm{BrS})$ poly(styrene-co-4-bromostyrene), $N$-TPI "new thermoplastic polyimide" (see reference), PPrA poly-n-propyl acrylate, PPeA poly$n$-pentyl acrylate; the rest of the polymer acronyms as usual

References: 1. Voigt-Martin et al. 1986; 2. Shaw 1974; 3. Takahashi et al. 1985; 4. Strobl et al. 1986; 5. Nojima et al. 1982; 6. Koningsveld et al. 1974; 7. McBrierty et al. 1978; 8. McMaster and Olabisi 1975; 9. Ougizawa and Inoue 1986; 10. Jagger et al. 1983; 11. Inoue et al. 1985; 12. Saito et al. 1987; 13. Goh et al. 1982; 14. Walsh et al. 1982; 15. Walsh and Rostami 1985; 16. Ougizawa et al. 1985; 17. Bhagwagar et al. 1994; 18. Kambour et al. 1980; 19. Hoy 1970; 20. Sauer et al. 1996; 21. Kim and Paul 1992; 22. Sato et al. 1996a, b; 23. Nishimoto et al. 1995; 24. Ougizawa et al. 1991; 25. Han et al. 1988; 26. Fernandes et al. 1986; 27. Hasegawa et al. 1992; 28. Woo et al. 1986; 29. Maiti and Nandi 1995; 30. Pennings and Manley 1996; 31. Krishnamoorti et al. 1995; 32. Kyu et al. 1991; 33. Sham and Walsh 1987; 34. Kim and Paul 1992; 35. Andradi and Hellmann 1993; 36. Gan and Paul 1994b; 37. Gan et al. 1994

\subsection{Experimental Methods}

The thermodynamic properties of a polymer blend determine its performance. Many authors have focused on miscibility - this aspect has been extensively discussed in the literature (Olabisi et al. 1979; Solc 1982; Utracki 1989; Coleman et al. 1991; Paul and Bucknall 2000; Koningsveld et al. 2001), and few selected results, with the emphasis of what is not covered in details in the following chapters, are briefly mentioned here. The characterization methods of the thermodynamic behavior of polymer blends can be divided into groups:

Thermodynamic studies not directly related to miscibility (e.g., PVT measurements)

Determination of the interaction parameter $\left(\chi_{12}\right.$ quantified through SAXS, SANS, melting point depression, from the Hess cycle, etc.)

Direct methods of miscibility determination (turbidity measurements, microscopy, combinatorial approaches, etc.)

Studies of the phase equilibria (determined by scattering methods, viz., turbidity, PICS, SAXS, and SANS; fluorescence techniques; ultrasonic measurements; etc.).

Indirect methods of miscibility determination (e.g., from the glass transition temperature, viz., $T_{g}$ from thermal, dielectric, or mechanical tests, NMR, spectroscopic methods, ODT through rheology, etc.) 


\subsubsection{PVT and Related Measurements}

Equilibrium thermodynamics controls the PVT behavior of any system and its thermal expansion coefficient, compressibility, bulk modulus, hardness, etc. The thermodynamic pressure, which can be defined as a partial derivative of the Helmholtz free energy (see Eq. 2.5), for multicomponent systems, comprises of two interaction parameters, e.g., $\left\langle\varepsilon^{*}\right\rangle\left\langle v^{*}\right\rangle^{k} ; k=2,4$. These values can as easily be determined from dilatometric measurements as from the phase diagram (Jain et al. 1982). With the advance of other methods, dilatometry has been largely neglected: It is still being used to characterize the compressibility of neat resins, but rarely nowadays to study the behavior of polymeric blends (Plochocki 1982, 1983, 1986; Zoller 1989; Steller and Zuchowska 1990; Zoller and Walsh 1995).

The interaction parameters can be calculated from the PVT data of polymer blends (Jain et al. 1982; Privalko et al. 1985); however, due to the need for subtraction of two large numbers, the resulting values were often erratic, with errors up to $\pm 6 \%$. Such errors may be acceptable for some applications, but not for the construction of phase diagrams. A more straightforward experimental route is the computation of the reducing parameters, $P^{*}, V^{*}$, and $T^{*}$, by, e.g., two possible approaches:

1. Experimental values are compared with theoretical predictions, assuming an EoS and an averaging scheme, e.g., Eq. 2.43. This approach was used, for example, to interpret the thermodynamic behavior of PS/PVME blends, for $T$, 25-200 ${ }^{\circ} \mathrm{C}$, and $P, 0-200 \mathrm{MPa}$ (Ougizawa et al. 1991), and for a series of polyolefins (EPR, a-PP, PP, PEP, PEB, i-PB, PIB, etc.) whose miscibility was studied using PVT and SANS measurements (Krishnamoorti et al. 1996). The results were interpreted using Hildebrand's solubility parameter formalism. For regular blends, a close agreement was found.

2. From $P^{*}, V^{*}$, and $T^{*}$, the molar attractive energy, $\varepsilon_{12}$, and repulsion volume, $v_{12}$, can be calculated and, subsequently, compared with theoretically derived averages, viz., the geometric mean for the former and the algebraic mean for radius for the latter (see Sect. 2.4.1). For miscible systems, the dependence of these values on composition can be easily derived. Usually, these compare reasonably well with the experimental data, since immiscibility causes large variations from the monotonic dependence.

Several interesting observations relate to such thermodynamic measurements. For example, the exothermic effects, associated with phase separation in LCSTtype polymer blends, showed a correlation between the exothermic enthalpy and the interactions between the components (Natansohn 1985); however, the specific interaction parameter $\chi_{12}$ was not calculated. In another example, there are definitive correlations between the thermodynamic and the transport properties (see Chap. 7, "Rheology of Polymer Alloys and Blends"). Thermodynamic properties of multiphase polymeric systems affect the flow, and vice versa. As discussed in - Chap. 7, "Rheology of Polymer Alloys and Blends", the effects of stress can engender significant shift of the spinodal temperature, $\Delta T_{s}=16^{\circ} \mathrm{C}$. While at low stresses the effects can vary, i.e., the miscibility can either increase or decrease, 
at higher stress values an enhancement of miscibility is expected. Flow has also been used to establish whether the molten blends are miscible or not (Schlund and Utracki 1987a, b, Utracki and Schlund 1987a, b).

Finally, thermodynamics also plays a dominant role in interfacial phenomena, viz., value of interfacial tension, thickness of the interphase, $\Delta l$, rheological properties, adhesion, compatibilization, etc. It is worth recalling that most lattice theories directly relate the magnitudes of $\gamma_{12}$ and $\Delta l$ to the value of the binary interaction parameter, $\chi_{12}$ (Helfand and Tagami 1971a, b, 1972; Helfand 1975a, b, c; Helfand and Sapse 1975; Roe 1975; Joanny and Leibler 1978; Broseta et al. 1990; Anastasiadis 2011). For example, the interfacial tension, as measured from pedant drop or scattering experiments, can be used to obtain binary interaction parameters, $\chi_{i j}$ (Anastasiadis 2011). In another example, the equilibrium interfacial thickness, $\Delta l$, in PMMA blends with PS and SAN was determined by ellipsometry (Higashida et al. 1995); from the $\Delta l$ values, the authors computed the temperature dependence of the binary interaction parameter, $\chi_{i j}(T)$, and then the phase diagrams. However, since these topics are of prime importance for Chap. 4, they will not be discussed here.

\subsubsection{Determination of Interaction Parameters}

\subsubsection{Binary Systems}

All types of radiation scattering techniques, viz., light, X-ray, and neutron, have been used to measure the interaction parameters and study the phase equilibria in polymer blends and solutions. Using the relations derived for polymer solutions (see Eqs. 2.27, 2.28, 2.29, 2.30, 2.31, and 2.32), scattering methods can be used to measure molecular weight, $M_{w}$; end-to-end distance or radius of gyration, $\left\langle s_{o}^{2}\right\rangle^{1 / 2}$; and the second virial coefficient, $A_{2}$, thus $\chi_{12}$.

The relation between the characteristic dimension of the scattering phase, $d_{a v}$; the wavelength of irradiation, $\lambda$; and the scattering angle, $\Theta$, is given by (see Eq. 2.71)

$$
d_{a v}=\Lambda=\frac{\lambda}{2} \sin (\Theta / 2)
$$

Depending on the experimental values of $\lambda$ and $\Theta$, the experimentally accessible values of $d_{a v}$ vary accordingly. Approximate ranges of the dimensions of scattering phases for the light, neutron, and X-ray techniques are given in Table 2.23.

The great majority of polymer blends have domain sizes in the range of $50 \mathrm{~nm}$ to $5 \mu \mathrm{m}$. Thus, LS and SAXS methods albeit more proliferated have limited use for studies of phase morphology, while SANS is much better suited. SANS has been used to study macromolecular size and conformation, morphology, in a single or multicomponent system, in molten or solid state. Since the contrast, phase discrimination, is based on the mass number, it is very useful to replace the hydrogen atoms in one polymer, or parts of a polymer, by deuterium. The deuterated polymer is mixed with its hydrogenated homologue at a selected low concentration, usually $\sim 0.1 \%$, providing a means to control contrast. The mixture can be then used as one of the 
Table 2.23 Spatial ranges for the scattering phase dimensions accessible to various scattering methods

\begin{tabular}{lll}
\hline Method & Origin of contrast & Scattering domain size $(\mu \mathrm{m})$ \\
\hline Light scattering (LS) & Refractive index & $1-100$ \\
\hline Small-angle neutron scattering (SANS) & Mass number & $0.01-3$ \\
\hline Small-angle X-ray scattering (SAXS) & Electron density & $1-100$ \\
\hline Wide-angle X-ray scattering (WAXS) & Electron density & $0.1-1$ \\
\hline
\end{tabular}

blend's components, or for the study of homopolymer properties. Several reviews of the SANS application for the characterization of polymer conformation and morphology have been published (Sperling 1984; Rennie 1992; Balsara et al. 1992; Lohse 1994; Krishnamoorti et al. 1995; Takeno et al. 1996; Hammouda 2010).

It should be noted that deuteration will change the conformation of macromolecules and also their miscibility/solubility, especially for high $M_{w}$ (Utracki 1989). Theory predicts phase separation (with UCST) for a blend of hydrogenated with deuterated polymer (Buckingham and Hentschel 1980; Edwards 1983; Honeycutt 1992a, b); see also Fig. 2.7, a behavior that has been experimentally observed in multiple systems (Eitouni 2007): Phase separation of poly-1,4-butadiene with its deuterated analog confirmed the prediction (Bates et al. 1985, 1986; Bates and Wignall 1986; Bates and Wilzinius 1989; Schelten et al. 1977; Yang et al. 1983; Atkin et al. 1984). Similarly, blends of hydrogenated with deuterated PDMS show immiscibility (Lapp et al. 1985). Apparently, there are three principle origins for these isotopic effects: (i) position of the isotope, (ii) conditions for SANS measurements, and (iii) difference in segmental volume upon deuteration. In conclusion, SANS is an excellent method for determination of molecular size and intermolecular interaction in polymer blends, provided that the isotopic effects are either absent or appropriately corrected for. The method is precise in quantifying the dependencies on molecular weight, molecular structure, macromolecular architecture, chemical substituents and additives, as well as on independent variables, $P, T$, deformation, etc. In Table 2.24, a few examples of blend studies are summarized. The interested reader is referred to consult specialized publications (Utracki 1989; Balsara et al. 1992; Lohse 1994; Krishnamoorti et al. 1995; Eitouni 2007; Hammouda 2010) or books.

SANS has been used extensively to determine $\chi_{12}$ of polymer blends, by fitting SANS profiles measured from blends to RPA. This approach was pioneered by Hadziioannou and Stein (1983, 1984), Murray and Stein (1985), and HerktMaetzky and Schelten (1983). In particular, the phase diagram in PVME/d-PS was among the first blends studied and has been extensively studied since then (Hadziioannou et al. 1983, 1984; Schwahn et al. 1987; Hammouda et al. 1995; Takeno et al. 1996; Choi et al. 1998, 2000; etc.), partly due to its well-documented LCST and the small difference in LCST $\left(\Delta T_{c r}^{L C S T} \approx 40^{\circ} \mathrm{C}\right)$ upon deuteration. In the vicinity of the critical point, a non-mean-field behavior was observed. The SANS results can be summarized as follows (Utracki 1989):

- The $M_{w}$ determined by the solution methods agreed with values obtained by SANS; the average ratio $M_{w}{ }^{\text {(sol) }}$ to $M_{w}{ }^{\text {(SANS) }}$ was found to be 1.02 ; 
Table 2.24 Example SANS studies of polymer blends ( $d$ before an acronym indicates a deuterated polymer). See also Table 2.8 for selected $\chi_{12}(T)$ based on SANS data

\begin{tabular}{|c|c|c|}
\hline Blend & Comment & References \\
\hline$d$-PS/PpMS & $\begin{array}{l}\text { For } 1: 1 \text { polystyrene/poly }(p \text {-methylstyrene }) \text { blend: } \\
1000 \chi_{12}=-(0.2 \pm 0.1)+(2160 \pm 60) / T\end{array}$ & 1 \\
\hline$d$-PS/PpMS & $M_{w}, \phi$, and $T$ dependence of $\chi_{12}$ & 2 \\
\hline $\mathrm{PS} / \mathrm{PoCS}$ & $\begin{array}{l}\text { PS/poly }(o \text {-chloro styrene) blends showed both LCST and } \\
\text { UCST; } \chi_{12} \text { was independent of } \phi \text { and } T\end{array}$ & 3 \\
\hline$d$-PB/PI & $\begin{array}{l}\chi_{12} \text { and phase diagrams for various blend compositions }(3 / 7 \text {; } \\
5 / 5 ; 7 / 3) ; \text { LCST from SANS and SALS }\end{array}$ & 4 \\
\hline $\mathrm{PVE} / \mathrm{PI}$ & $\begin{array}{l}\text { QENS showed PI dynamics same as in homopolymer, but PVE } \\
\alpha \text {-relaxation plasticized by PI }\end{array}$ & 5 \\
\hline LDPE/HDPE & Studies of PE/PE miscibility & 6 \\
\hline $\mathrm{PE} / \mathrm{PE}$ & $\begin{array}{l}\text { Blends of linear-PE/branched-PE showed difficulties in } \\
\text { interpretation of SANS data }\end{array}$ & 7 \\
\hline$d$-PE/EEA & $\begin{array}{l}\text { PE/EEA ( } 18 \% \text { EA copolymer) showed immiscibility } \\
\text { (EA domains of } 3-4 \mathrm{~nm} \text { ) }\end{array}$ & 8 \\
\hline $\mathrm{PIB} / \mathrm{PP}$ and $\mathrm{EB}$ & Composition and $T$ dependence of $\chi_{12}(\phi, T)$ & 9 \\
\hline $\begin{array}{l}\mathrm{PIB} / \mathrm{PP} \text { and } \mathrm{EB}, \\
d \text {-PS/PS }\end{array}$ & Compressibility effects, cf. Sanchez-Lacombe theory & 10 \\
\hline $\mathrm{PP} /$ polyolefins & Isotopic, $\phi$, and $T$ effects on $\chi_{12}$ & 11 \\
\hline Polyolefin blends & PP/EPR, etc., comparison between SANS and $P V T$ data & 12 \\
\hline Polyolefin blends & $\begin{array}{l}\text { LDPE/HDPE immiscible at } 143{ }^{\circ} \mathrm{C} \text { with } \chi_{12}=+0.00056 \text {; } \\
d \text {-HDPE } / d \text {-PB had } \chi_{12}=+0.0004 ; \mathrm{PB} / d \text {-PB had } \\
\chi_{12}=+0.00053 \text { at } 130{ }^{\circ} \mathrm{C}\end{array}$ & 13 \\
\hline Polyolefin blends & $\begin{array}{l}\mathrm{PP} / \text { poly(ethylene-co-1-hexene): } \chi_{12} \text { showed a min at } 50 \% \\
\text { 1-hexene comonomer }\end{array}$ & 14 \\
\hline $\mathrm{PMB} / \mathrm{PEB}$ & $\begin{array}{l}\text { Poly(methyl butylene)/poly(ethyl butylene) binary and ternary } \\
\text { systems; } \chi_{12}=0.0028-2.30 / T+584.45 / T^{2}\end{array}$ & 15 \\
\hline $\mathrm{PMB} / \mathrm{PEB}$ & $P$ dependence of $\chi_{12}(T)\left(\chi_{12}\right.$ becomes $\propto 1 / T$ at higher $\left.P\right)$ & 16 \\
\hline $\begin{array}{l}d \text {-PS/PVME, } d \text {-PB/ } \\
\mathrm{PB}, d \text {-PB/PI }\end{array}$ & $\begin{array}{l}\text { Blends studied over various } T \text { regions (regions } \gg T_{g}, \sim T_{g} \text {, } \\
\text { and }<T_{g} \text { ) }\end{array}$ & 17 \\
\hline $\mathrm{PP} / \mathrm{PI}$ & $\begin{array}{l}\chi_{12}(\phi, T) \text { of binary and ternary blends of head-to-head PP with } \\
\text { head-to-tail PP and PI }\end{array}$ & 18 \\
\hline
\end{tabular}

References: 1. Jung and Fischer 1988; 2. Londono and Wignall 1997; 3. Murray et al. 1985; 4. Hasegawa et al. 1991; 5. Arbe et al. 1999; 6. Londono et al. 1994; 7. Schipp et al. 1996; 8. Marr 1995; 9. Krishnamoorti et al. 1995; 10. Taylor et al. 1996; 11. Graessley et al. 1995; 12. Krishnamoorti et al. 1996; 13. Alamo et al. 1997; 14. Seki et al. 2000; 15. Lin et al. 1996; 16. Lefebvre et al. 2000, 2002; 17. Takeno et al. 1996; 18. Reichart et al. 1997

- Increasing the blends' temperature causes the second virial coefficient, $A_{2}$, to decrease, thus, leading to an LCST as a rule for the studied systems.

- The radius of gyration, $\left\langle s_{o}^{2}\right\rangle^{1 / 2}$, of the deuterated polymer decreased with $M_{w}$, in the matrix of the same chemical character. In most cases, the coil size of the probe molecules was found to be slightly increasing with $A_{2}$.

- Given the small positive values of $A_{2}$, blends of PMMA/SAN, PVC/PMMA, PPE/PS, and PVME/PS are miscible. However, in each system, the value of $A_{2}$ 
decreased with $M_{w}$, indicating worsening miscibility. In these systems $\left\langle s_{o}^{2}\right\rangle^{1 / 2} \propto$ $M_{W}^{n}$, with the exponent $n$ depended on the system and temperature. Specifically, for PS/PVME, $n=0.57\left(25^{\circ} \mathrm{C}\right)$ or $0.52\left(120^{\circ} \mathrm{C}\right)$; for PPE/PS, $n=0.55$; whereas, for PS in PS and for PMMA in PMMA, $n=0.5$; and for PMMA in PSAN-19, $n=0.60$, and for PSAN-19 in PMMA, $n=0.64$.

SAXS and SANS were used to study PMMA with PVDF blends (Wendorff 1980, 1982; Hadziioannou and Stein 1984). The binary interaction parameter $\chi_{12}$ was plotted versus PVDF content, and the isothermal data (at $T=200{ }^{\circ} \mathrm{C}$ ) could be expressed by a linear dependence (Wendorff 1980, 1982): $-1 /\left(\chi_{12}+0.0035\right)=-0.72$ $+0.76 \phi_{P V D F}$. Several authors reported data of $\left\langle s_{o}^{2}\right\rangle^{1 / 2}$ and $A_{2}$ for polymer blends (Hadziioannou et al. 1983; Ree 1987; Maconnachie et al. 1984). The binary interaction parameter $\chi_{12}$ can be extracted from the second virial parameter $A_{2}$. As discussed in Sect. 2.5.1, $\left\langle s_{o}^{2}\right\rangle^{1 / 2}$ and $A_{2}$ are measured using scattering methods, including light scattering (LS). One innovative way of using LS involves polymer/polymer/solvent ternary systems: This approach requires either that one of the polymers is iso-refractive with the solvent or that the polymers have equal contrast (Pinder 1997). The method was successfully used to measure $\chi_{12}$ for PS/PMMA blends of different $M_{w}$. Similarly, $A_{2}$ can be determined from osmotic pressure measurements of polymer/polymer/solvent ternary systems, yielding $\chi_{12}(\phi)$ for PVCVAc with acrylic copolymers in cyclohexanone (Sato et al. 1997).

SAXS has been mainly used to study morphology of the semicrystalline blends, cf. how it is affected by composition, crystallization rate, compatibilization, additives, etc. However, it can also be used to study local structures in molten polymer blends, for example, within the interphasial region. The method has been used for liquid, glassy, and crystalline systems to determine the spinodal and binodal temperatures as well as to measure $\chi_{12}$. A reasonable agreement between the values measured by different methods was obtained (Harris et al. 1983; Riedl and Prud'homme 1984; Barlow and Paul 1987).

The depression of the melting point, $T_{m}$, has also been used to determine $\chi_{12}$. Development of the method is credited to Nishi and Wang $(1975,1977) . T_{m}$ depends on two factors: (1) the unit cell geometry, as well as the type and dimensions of the crystals/crystallites, and (2) the interactions between the crystalline polymer and other ingredients. To determine $\chi_{12}$ from $T_{m}$, it is important that there are no chemical reactions and all specimens (e.g., representing different compositions) are identically treated (identical thermal history), as well as that the mutual solubility of low molecular weight fractions is either small or independent of the blend composition.

However, it is important to ascertain that incorporation of other ingredients changes crystallinity only through thermodynamic interactions, while other effects on crystallinity are negligibly small. Blending can affect crystallinity in diverse ways, due to the effects of added components on nucleation and growth rates. Thus, blending method and parameters, especially rates, can have serious effects on crystallizability and crystal size. Experimentally, the presence of a miscible amorphous polymer in the blend usually slows down, or even prevents, crystallization of the semicrystalline polymer. For fewer systems, enhancement of crystallinity and increase in $T_{m}$ upon blending have also been reported (Harris and Robeson 1987; 
Table 2.25 Examples of determination of $\chi_{12}$ from melting point depression studies

\begin{tabular}{|c|c|c|}
\hline Blend & Comment & References \\
\hline PCL/PVDC- $x$ & $\begin{array}{l}\text { PCL blends with PVDC, PVDC-VC, PVDC-VAc, or PVDC- } \\
\text { AN: } \chi_{12}\left(\phi_{P C L}\right) \text { showed a maximum at ca. } 30 \mathrm{wt} \% \text { and small } \\
T \text { dependence }\end{array}$ & 1 \\
\hline PVDF/acrylates & $\begin{array}{l}\text { PVDF blends with PMMA, PEMA, PMHA copolymers: } B_{12} \\
\text { was negative for all PVDC/acrylate blends }\end{array}$ & 2 \\
\hline PVDF/PMMA & $\begin{array}{l}\text { PVDF blends with PMMA and review of procedures used to } \\
\text { determine } \chi_{12}\end{array}$ & 3 \\
\hline $\begin{array}{l}\text { PVDF/PMA } \\
\text { PVF-VDF/PMA }\end{array}$ & $\begin{array}{l}T \text { dependence of } \chi_{12}(\phi) \text { was determined; miscibility turns into } \\
\text { immiscibility with increased PVF content }\end{array}$ & 4 \\
\hline $\mathrm{PVDF} / \mathrm{PBA}$ & $\begin{array}{l}\text { PVDF blends with poly(1,4-butylene adipate) were miscible } \\
\text { over full range of compositions: } \chi_{12}=-0.19\end{array}$ & 5 \\
\hline $\mathrm{PA} / M$-sPS & $\begin{array}{l}\text { PA blends with Mn- or Zn-sulfonated PS were miscible (with } \\
\chi_{12}<0 \text { ); results confirmed by FTIR and SAXS }\end{array}$ & 6 \\
\hline $\mathrm{PBT} / \mathrm{PAr}$ & $\begin{array}{l}\text { PAr depressed PBT's } T_{m} \text { by } 17^{\circ} \mathrm{C} \text {; calculated } \chi_{12} \text { varied from } \\
-0.65 \text { (20 wt } \% \text { PAr) to }-0.22(80 \mathrm{wt} \% \text { of PAr })\end{array}$ & 7 \\
\hline PBT/ester-ethers & $\begin{array}{l}\text { PBT blends with segmented/block poly(ester-co-ether): } \\
\text { miscibility depended on the copolymer composition }\end{array}$ & 8 \\
\hline $\mathrm{PA} / \mathrm{MXD}$ & $\begin{array}{l}\text { PA-g blends with poly }(m \text {-xylene adipamide }): \chi_{12}<0 \text { indicated } \\
\text { miscibility in amorphous phase; miscibility increased with } \\
\text { transamidation during aging. }\end{array}$ & 9 \\
\hline $\begin{array}{l}\text { PP/SEBS/oil } \\
\text { PP/EPR/oil }\end{array}$ & $\begin{array}{l}\text { Miscibility was concluded for PP/SEBS/oil, } \chi_{12}=-0.043 \text {, and } \\
\text { immiscibility for PP/EPR/oil }\end{array}$ & 10 \\
\hline PCL/P4HS & $\begin{array}{l}\text { PCL blends with poly(4-hydroxy styrene): } \chi_{12} / V=-0.013 \text { and } \\
\text { single } T_{g} \text { indicated miscibility }\end{array}$ & 11 \\
\hline CR/PEMA & $\begin{array}{l}\text { Miscibility concluded from the negative } \chi_{12}:-0.030 \text { to }-0.122 \text {, } \\
\text { and from FTIR }\end{array}$ & 12 \\
\hline PET/PETG & Miscibility concluded from the negative $\chi_{12}=-0.122\left(280^{\circ} \mathrm{C}\right)$ & 13 \\
\hline
\end{tabular}

References: 1. Zhang and Prud'homme 1987; 2. Goh and Siow 1988; 3. Runt and Gallagher 1991; 4. Maiti and Nandi 1996; 5. Pennings and Manley 1996; 6. Lu and Weiss 1991, 1992; 7. Huo and Cebe 1993; 8. Gallagher et al. 1993; 9. Shibayama et al. 1995; 10. Ohlsson and Tørnell 1996; 11. Lezcano et al. 1996; 12. Kundu et al. 1996; 13. Papadopoulou and Kalfoglu 1997

Dumoulin et al. 1987). As a result, despite its simplicity, obtained values of $\chi_{12}$ from the $T_{m}$ method should be confirmed by other techniques (Utracki 1989; Groeninckx et al. 1998). Enthalpic interaction parameters determined for low molecular analogs via direct calorimetric measurements of the enthalpy of melting, $\Delta H_{m}$, provide data which correlate well with $\chi_{12}$ determined by other methods (Barlow and Paul 1987; Rana et al. 1996; Ziaee and Paul 1996, 1997). The most crucial aspect here is the selection of suitable analogs. In this task, consideration of the partial charges of the atoms in each molecule can be used as guide (Ziaee and Paul 1996, 1997); as discussed in the heat of mixing Sect. 2.6.2.2, there are several disadvantages in such an approach, for example, inability to account for structural and/or polydispersity effects. Another method that can address some of these concerns is the microcalorimetric determination of $\Delta H_{m}$ using low viscosity oligomeric mixtures (Singh and Walsh 1986; Sham and Walsh 1987) (Table 2.25). 


\subsubsection{Ternary Systems Containing Solvent}

The difficulties in the calorimetric determination of the interaction parameters are caused by the high viscosity of most commercially relevant or academically interesting polymers and the accompanying slow diffusion rates, heat generation during mixing or processing, etc. These problems do not exist for solutions.

One way to overcome such problems is to consider solvent(1)/polymer(2)/ polymer(3) ternary systems; any method that determines either $\Delta G_{m}$ or its derivatives should make it possible to calculate $\chi_{23}$. Thus, for example, osmotic pressure measurements were used to characterize PS/PVME blends dissolved in either toluene or ethylbenzene (Shiomi et al. 1985). The $\chi_{23}$ was found to depend on the blends' composition. Elimination of the solvent effects gave $\chi_{23} / V_{1}=-10^{4}$ $\left(7.41-11.01 \phi_{3}\right)$. Thus, the system was expected to remain miscible up to a PVME volume fraction of $\phi_{3}=0.67$. Osmotic pressure has also been used to determine $\chi_{23}=0.070$ for PS with poly( $p$-chloro styrene) in toluene, 2-butanone, and cumene (Ogawa et al. 1986). For the same system, $\chi_{23}=0.087$ was calculated from intrinsic viscosity measurements. Thus, the system is thermodynamically immiscible. More recently, osmotic pressure measurements in cyclohexanone of a ternary system resulted in $\chi_{23}(\phi)$ for poly(vinylchloride-co-vinylacetate) blends with a series of acrylic copolymers (Sato et al. 1997).

Vapor sorption of PS/poly $\left(\alpha\right.$-methylstyrene) gave $\chi_{23}=0.504$, varying with $T$ and polymer concentration, indicating that this system is immiscible with UCST $>100{ }^{\circ} \mathrm{C}$ (Saeki et al. 1981). Light-scattering measurements of ternary systems, polymer(1)/polymer(2)/solvent(3), were also successfully used to determine polymer/polymer interaction parameters, $\chi_{12}(\phi)$. The method is particularly easy to use either if one of the two polymers is iso-refractive with the solvent or if the polymers have equal contrast (Pinder 1997). The method was successfully used to measure $\chi_{12}$ for PS/PMMA blends of different $M_{w}$.

Over the years, several authors tried to correlate polymer/polymer miscibility with solution viscosity in a common solvent (e.g., Bohdanecky and Kovar 1982). An interesting report in this field was (Chee 1990) considered that the parameter $b=k_{H}[\eta]^{2}\left(k_{H}\right.$ is the Huggins constant of Eq. 2.33, and [ $\left.\eta\right]$ is the intrinsic viscosity) can be set as a measure of the interactions between the solvent and the polymeric species. For polymer blends, the author wrote

$$
\left.\begin{array}{l}
{[\eta]_{\text {blend }}=w_{2}[\eta]_{2}+w_{3}[\eta]_{3}} \\
b_{\text {blend }}=w_{2}^{2} b_{22}+w_{3}^{2} b_{33}+2 w_{2} w_{3} b_{23}
\end{array}\right\} \Rightarrow \mu \equiv \frac{\left[b_{23}-\left(b_{22}+b_{33}\right) / 2\right]}{\left([\eta]_{3}-[\eta]_{2}\right)^{2}}
$$

where the parameter $\mu$ as defined in Eq. 2.75 is a measure of the polymer/polymer miscibility (viz., negative $\mu$ values indicate immiscibility, and positive $\mu$ miscibility). Three series of blends were examined: (1) PVC/PMMA, (2) PiBMA/PMMA, and (3) PiBMA/PVC. In agreement with the calculated values of the parameter $\mu$, the first of these three blends was found miscible, whereas the two other immiscible in the full range of composition. However, the method is, at best, qualitative. For example, the effect of the common solvent on the parameter $\mu$ was not investigated, 
but fundamentals of intermolecular interactions make it dubious that nonpolar solvents will lead to the same value of the parameter $\mu$ as strongly polar ones. The author observed that the method breaks down for polymer pairs that can form associations. Intrinsic viscosity measurements were also used to evaluate intermolecular interactions in blends of cellulose diacetate with poly(vinyl pyrrolidone) (Jinghua et al. 1997).

Another method is based on the principle that the change in any thermodynamic state function depends only on the initial and final states (Hess cycle). For example, in path I, two polymers are dissolved separately in the same solvent and then mixed together; in path II, polymers are first blended together and then dissolved in the same solvent. From the balance of the dissolution enthalpies, the heat of mixing of two polymers, $\Delta H_{m}$, can be calculated at the corresponding temperature. However, since $\Delta H_{m}$, in the above example, is a small number determined by subtracting two large values from each other, the error of these estimation can be large. Furthermore, $\chi_{23}$ determined from $\Delta H_{m}$ above is from dilute systems, and its extrapolation to melt may be impractical (Koningsveld et al. 1974). $\Delta H_{m}$ measurements have been used to characterize PPE blends with either PS, halogenated PS, or copolymers (Zacharius et al. 1983). At $34.8{ }^{\circ} \mathrm{C}$, the heat of mixing of PS/PPE blend was small and negative, indicating weak specific interactions. By contrast, in blends of PS with poly(2-chloro styrene), $\Delta H_{m}$ was small and positive, dependent on molecular weight and temperature. In agreement with the observations, for PS/poly (2-chloro styrene-co-4-chloro styrene), $\Delta H_{m}=0.31 \mathrm{~J} / \mathrm{g}$ was found in the full range of copolymer compositions, confirming immiscibility.

Size exclusion chromatography, SEC, has also been used for the determination of polymer/polymer interaction coefficients in solvent/polymer/polymer threecomponent systems (Narasimhan et al. 1979, 1983, 1984). The method was found precise and thermodynamically significant. Strong solvent concentration dependence of $\chi_{23}>0$ was reported from tests of toluene/PMMA/PS system (Lau et al. 1984, 1985).

In conclusion, it is important to note that the determination of $\chi_{23}$ is of dubious value for predicting polymer/polymer miscibility, especially for processing conditions. The chi parameter is a complex function of many variables, including $T$ and $P$ that can become extreme during processing. The solution methods require high polymer dilution and low temperatures, significantly lower than those used for compounding or forming of polymer blends. Methods capable to accurately extrapolate solution data to the processing conditions do not exist. The above comments are pertinent to any of the ternary solvent methods of $\chi_{23}$ determination.

\subsubsection{Phase Diagrams}

\subsubsection{Turbidity Measurements}

The method consists of preparation of a series of mixtures of varied concentrations (near the phase separation condition) then causing the separation to occur, e.g., by ramping the temperature. The onset of turbidity is observed visually, using 
a photoelectric cell, or by a UV-visible spectrophotometer. The ensemble of the cloud points defines the cloud-point curves (CPC) that closely follow the system's binodal. The method can be extended to rigorous studies of phase separation by measuring the light-scattering intensity.

For polymer blends, the CPC is usually determined by preparing films under conditions of miscibility, in a wide range of compositions. The films are then heated through the cloud point at a rate not exceeding $0.1{ }^{\circ} \mathrm{C} / \mathrm{min}$. Depending on the rate, type of system, and polydispersity, the hysteresis (difference between CPC on heating and cooling) can be significant. Examples of blends whose phase diagrams were determined are listed in Table 2.26.

The scattered intensity of light due to concentration fluctuations, extrapolated to zero-scattering angle, is inversely proportional to the second derivative of $\Delta G_{m}$. Thus, it can be used to determine the location of a spinodal, i.e., the spinodal temperature, $T_{s}$, for the given mixture. As Eq. 2.32 indicates, LS makes it possible to determine also the second virial coefficient $\left(A_{2}\right)$ and from it the binary interaction parameter $(\chi$ or $B)$. However, this technique is applicable only to homogenous systems, i.e., at temperatures $T \leq T_{s}$ for LCST systems or at $T \geq T_{s}$ for those having UCST. As mentioned in Sect. 2.8.2.2, the LS methods has been used primarily to study the phase equilibria of polymer solutions.

Pulse-induced critical scattering, PICS, is an elegant method of LS measurements that makes it possible to extend the measurements closer to the spinodal. It uses a small mass of a homogenous liquid mixture very rapidly heated or cooled into the metastable region. The laser light-scattering intensity is measured after thermal equilibrium is reached, but before the system can phase separate, the mixture is brought out into the homogenous region and the cycle repeated (Gordon et al. 1973). The temperature change can be accomplished in milliseconds, afforded by the small specimen size, and the time of one full cycle is less than a minute.

One of the most serious obstacles in the phase equilibrium studies of polymer blends is the viscosity of the system. At the accessible temperatures, between softening point and thermal degradation, the self-diffusion coefficient of macromolecules is of the order of $10^{-4}$ to $10^{-6} \mathrm{~m}^{2} / \mathrm{s}$ (Kausch and Tirrell 1989). As a result, phase separation is very slow. To accelerate the process, a low-speed centrifuge, the "centrifugal homogenizer" (CH), with PICS has been used (Koningsveld et al. 1982). In short, centrifugation within the immiscibility zone permits determination of binodal and critical points, while use of the PICS mode allows location of the spinodal.

\subsubsection{Scattering Methods}

Turbidity, light scattering, and PICS methods, discussed in the preceding paragraphs, are based on the scattering of light by liquid systems with optical heterogeneities. These principles have been extended to other types of radiation, e.g., X-rays and neutrons, cf. SAXS and SANS, which have been used to study polymer blend structures. In contrast to light scattering, SAXS uses the regularity of crystalline, or pseudocrystalline arrays of atoms, whereas SANS that of different mass of atoms. The data are treated via a relation derived for the conventional lightscattering equation (Eq. 2.32): 
Table 2.26 Examples of polymer blends with known phase diagram(s)

\begin{tabular}{|c|c|c|}
\hline Blend & Comment & References \\
\hline \multirow[t]{5}{*}{ PS/PVME } & \multirow{5}{*}{$\begin{array}{l}\text { LCST. A rare case of miscible } \\
\text { homopolymers. } M_{w} \text { only slightly } \\
\text { affects the critical concentration } \\
\text { (about } 10 \mathrm{wt} \% \text { of PS), but strongly } \\
\text { changes the critical temperature } \\
102-145^{\circ} \mathrm{C}\end{array}$} & Nishi and Kwei 1975 \\
\hline & & Nishi et al. 1975 \\
\hline & & Reich 1986 \\
\hline & & Qian et al. 1991 \\
\hline & & Radusch et al. 1996 \\
\hline \multirow[t]{3}{*}{ SAN/PMMA } & \multirow{3}{*}{$\begin{array}{l}\text { LCST. PMMA miscibility with SAN } \\
(5.7-38.7 \mathrm{wt} \% \mathrm{AN} \text {; at } \\
\left.T=140-170{ }^{\circ} \mathrm{C}\right) . \text { Interfacial } \\
\text { thickness data. PMMA is immiscible } \\
\text { with both PS and PAN }\end{array}$} & McMaster 1975 \\
\hline & & McBrierty et al. 1978 \\
\hline & & Higashida et al. 1995 \\
\hline \multirow[t]{4}{*}{$\mathrm{SAN} / \mathrm{PCL}$} & \multirow{4}{*}{$\begin{array}{l}\text { LCST. Miscibility chimney } \\
\text { dependent on the blend composition } \\
\text { and AN content in SAN }\end{array}$} & McMaster 1973; \\
\hline & & Schulze et al. 1993; \\
\hline & & Kammer et al. 1996 \\
\hline & & Higashida et al. 1995 \\
\hline $\mathrm{PS} / 4 \mathrm{MPC}$ & $\mathrm{LCST}=220^{\circ} \mathrm{C}$ & Shaw 1974 \\
\hline \multirow[t]{2}{*}{ PVDF/i-PEMA } & \multirow[t]{2}{*}{$\mathrm{LCST}=220^{\circ} \mathrm{C}$} & Saito et al. 1987 \\
\hline & & Hahn et al. 1987 \\
\hline $\mathrm{PMMA} / \alpha-\mathrm{MSAN}$ & $\mathrm{LCST}=185^{\circ} \mathrm{C}$ & Goh et al. 1982 \\
\hline PMMA/CPE & $\mathrm{LCST}=100{ }^{\circ} \mathrm{C}$ & Walsh et al. 1982 \\
\hline PMMA/PVC & $\mathrm{LCST}=190^{\circ} \mathrm{C}$ & Jagger et al. 1983 \\
\hline \multirow[t]{2}{*}{ PMMA/Phenoxy } & \multirow{2}{*}{$\begin{array}{l}\mathrm{LCST}=158{ }^{\circ} \mathrm{C}(30 \mathrm{wt} \% \text { phenoxy }) . \\
\text { Phase diagram from turbidity }\end{array}$} & Chiou and Paul 1991; \\
\hline & & Etxeberria et al. 1997 \\
\hline PES/Phenoxy & $\begin{array}{l}\mathrm{LCST}=194^{\circ} \mathrm{C}(57 \mathrm{wt} \% \text { of } \\
\text { phenoxy })\end{array}$ & Walsh and Singh 1986 \\
\hline PES/PEO & $\mathrm{LCST}=80^{\circ} \mathrm{C}$ & Walsh and Rostami 1985 \\
\hline PVC/NBR/plasticizer & $\begin{array}{l}\text { Miscibility only for the PVC/AN } \\
\text { part }\end{array}$ & Inoue et al. 1985 \\
\hline \multirow[t]{3}{*}{ PVC/Acrylates } & $\mathrm{LCST}=106^{\circ} \mathrm{C}$ for $\mathrm{PVC} / \mathrm{PPrA}$ & \multirow[t]{3}{*}{ Sham and Walsh 1987} \\
\hline & $\mathrm{LCST}=127^{\circ} \mathrm{C}$ for $\mathrm{PVC} / \mathrm{PBA}$ & \\
\hline & $\mathrm{LCST}=131^{\circ} \mathrm{C}$ for $\mathrm{PVC} / \mathrm{PPeA}$ & \\
\hline PS/PMPS & $\mathrm{UCST}=103{ }^{\circ} \mathrm{C}$ & Takahashi et al. 1986 \\
\hline SBR-45/BR & $\mathrm{UCST}=140{ }^{\circ} \mathrm{C}$ & Ougizawa et al. 1985 \\
\hline NBR-40/SAN & $\mathrm{UCST}=140{ }^{\circ} \mathrm{C}$ & Ougizawa and Inoue 1986 \\
\hline
\end{tabular}

$$
\frac{K c_{2}}{R(q)}=\frac{A_{1}}{M_{w, 2} P(q)}+2 A_{2} c^{2}+\ldots
$$

where $q \equiv(4 \pi / \lambda) \sin \theta$ is the scattering vector (cf. Eq. 2.71$) ; c_{2}$ is the polymer concentration, $M_{W, 2}$ the polymer molecular weight, and $A_{2}$ the polymer second virial coefficient (the subscript 2 indicates that the polymer is present in lower concentration); $R(q) \equiv I(q) \omega^{2} / I_{o} V_{s c}$ is the ratio of scattered to incident intensities (Rayleigh ratio); $P(q)$ is Debye's one-particle scattering form function, same with the one used in light scattering; $\lambda$ is the neutron wavelength; and $\theta$ is the scattering 
half-angle. The constant $K$ contains the scattering lengths of hydrogen $\left({ }^{1} \mathrm{H}\right)$ and deuterium $\left({ }^{2} \mathrm{H}\right)$ as the most important quantities, whereas the average scattering radius of the polymer, cf. $R_{g}^{s c}$ or $\left(R_{g}^{2} s c / M_{W}\right)^{1 / 2}$, is calculated from $P(q)$.

The phase equilibria in systems containing di-block poly(styrene- $b$-butadiene), $\mathrm{SB}$, mixed with either a homopolymer or a random copolymer were established by plotting the reciprocal of the intensity of the main SAXS peak as a function of 1/T; thus, the spinodal, $\mathrm{T}_{\mathrm{s}}$, and binodal, $\mathrm{T}_{\mathrm{B}}$, temperatures were measured ( $\mathrm{Zin}$ and Roe 1984). SAXS has been typically used to study the morphology of polymer blends in the solid state (Khambatta 1976; Russel, 1979; Russel and Stein, 1982, 1983). For example, in the interlamellar regions of PCL/PVC blend, the system is miscible on a molecular scale. Addition of PVC impeded crystallization of PCL. At high PVC concentration, PCL remained in solution. The radius of gyration was larger than that under unperturbed conditions, in spite of the fact that the second virial coefficient, $A_{2}$, was virtually zero. SAXS was also used to study the morphology of LDPE/HDPE blends (Reckinger et al. 1984, 1985). It was found that during the crystallization, macromolecules segregate. This segregation was also observed during rapid quenching at about $100{ }^{\circ} \mathrm{C} / \mathrm{min}$; at the high rates associated with the process, the segregation distance was comparable to coil dimension in the melt.

\subsubsection{Fluorescence Techniques}

"Excitation fluorescence" is the principle of the fluorescence techniques used for studying polymer blends. The method comprises of three steps: incorporation of an excimer, its excitation, and recording the excitation delay. The excimer can be an aromatic polymer component of the blend (viz., PS, poly(vinyl-dibenzyl), polyvinylnaphthalene, an aromatic group grafted onto the macromolecular chain, etc.), or it can be added as "probe" molecule (e.g., anthracene). There are three possibilities for the aromatic rings to form excimers: intramolecular adjacent, intramolecular nonadjacent, and intermolecular types. Each of these types is sensitive to different aspects of the chain conformation and environment, thus, sensitive to blend miscibility effects. The most important of these for studies of polymer blends is the intermolecular, usually identified from concentration measurements (Winnik et al. 1988).

In a second method, the "non-radiative energy transfer" method (NRET), the energy is transferred from a donor to a receptor chromophore, when the distance between them is of the order of $2-5 \mathrm{~nm}$. Phase separation is concluded from a decrease of the chromophore energy transfer. The method has been used to study PVC miscibility with PMMA or with SAN; PS or poly- $\alpha$-methylstyrene (P $\alpha$ MS) with PS- $\alpha$ MS copolymer; PS or P $\alpha$ MS or PBS [poly(tert-butyl styrene)] or PS + PBS with PS-BS copolymer; etc. (Morawetz 1980, 1981, 1983; Albert et al. 1986).

\subsubsection{Ultrasonic Velocity}

For homogenous systems, the ultrasonic velocity is related to the ratio of modulus to density. Thus, one may expect that any method that determines density changes with adequate precision can provide a measurement or an indication of 
miscibility (Singh and Singh 1983). The ultrasonics can also be used as a fast screening method for the optimization of processing and its parameters, e.g., employed as online characterization of polymers blends (Piau and Verdier 1993; Verdier and Piau 1995; Gendron et al. 1995).

The compressive ultrasonic velocity (6 MHz, at room temperature) was used to study cast blend films of PMMA/PVAc, PMMA/PS, PVC/CR, and PS/EPDM (Singh and Singh 1983; Shaw and Singh 1987). A linear correlation between the sound velocity and the composition was observed for miscible blends, whereas immiscibility, viz., in PMMA/PS blends, the same dependence was irregular. Phase separation in PVC/CR was detected at $w=70 \mathrm{wt} \%$ of CR, indicated by a sudden departure from linear correlation. The ultrasonic absorption versus composition gave even stronger evidence of immiscibility. Ultrasonics have been also successfully used to study the phase behavior in polyurethanes (Volkova 1981).

Acoustic emission has been frequently used in studies of the fracture behavior of fiber-reinforced composites. This method was also adopted to studies of blends. Since the sound is most frequently generated by debonding of two phases, there should be a drastic difference in the acoustic activity for blends located on the two sides of spinodal. To quantify miscibility between PVC and EVAc, acoustic emission measurements during a peel test of a-PVC/EVAc/PVC sandwich were carried out (Muniz et al. 1992). The authors considered that the acoustic emissions at slow rates of peeling are related not to the viscoelastic dissipation processes, but rather to the work necessary to pull apart polymeric chains or break bonds. The highest acoustic emission was obtained for VAc content in EVAc of 18 and $29 \mathrm{wt} \%$.

\subsubsection{Indirect Methods for Polymer/Polymer Miscibility}

These methods do not provide data for the binodal, spinodal, or the numerical value of the interaction parameter, but general information about the polymer/polymer miscibility. However, the information can frequently be used, e.g., to construct a map of miscibility - a simplified phase diagram.

\subsubsection{Glass Transition Temperature $\left(T_{g}\right)$ Measurements}

When polymer is cooled, from either the liquid or rubbery state, its molecular motion slows down, and eventually it undergoes a glass transition, or vitrification, preceeded by crystallization for semicrystalline polymers. The glass formation is a nonequilibrium phenomenon and is kinetic in nature, i.e., not a genuine first-order thermodynamic transition; thus, its characteristic temperature, $T_{g}$, is detected at different temperature values depending on the cooling rate, the probing method, the thermal history, etc. Nevertheless, most theoretical treatments consider the glass to be at a pseudo-equilibrium state, endowing $T_{g}$ with characteristics of a critical temperature of a second-order thermodynamic transition. The thermodynamics of such a state demands knowledge of "order parameters," $z_{i}$ : 


$$
d G=\left(\frac{\partial G}{\partial T}\right)_{P, z_{i}} d T+\left(\frac{\partial G}{\partial P}\right)_{T, z_{i}} d P+\sum_{i=1}^{n}\left(\frac{\partial G}{\partial z_{i}}\right)_{P, T, z_{j}} d z_{i}
$$

At equilibrium, the affinity coefficients, $A_{i} \equiv\left(\partial G / \partial z_{i}\right)_{P, T, z_{j}} \rightarrow 0$, and the equilibrium equation, Eq. 2.11, regain validity.

When liquids are viewed as collections of inherently non-crystallizable macromolecules, they are expected to show equilibrium amorphous properties in all $T$ ranges. Furthermore, in the thermodynamic description of the glassy state, it was postulated that cooling a liquid causes its configurational entropy to decrease, becoming zero at $T_{g}$ (DiMarzio and Gibbs 1958; Dong and Fried 1997). This concept gives legitimacy to the pseudo- or semi-equilibrium theories of the glassy state, viz., Couchman's theories (Couchman 1978, 1979a, b). It is widely accepted that when the test methods are slow enough (usually less than $1{ }^{\circ} \mathrm{C} / \mathrm{min}$ and/or $1 \mathrm{~Hz}$ ) the glass behaves in a semi-equilibrium manner and its behavior can be generalized.

In a first approximation, the polymer's glass transition is related to the cooperative segmental motion involving 50-100 backbone chain carbon atoms, or 15-30 statistical segments, i.e., a domain of a size $d_{d}=2-3 \mathrm{~nm}$ (Boyer 1966; Warfield and Hartmann 1980). However, the glass transition is not a phenomenon occurring at constant free volume. Along these lines, the most common use of $T_{g}$ in determination of polymer/polymer miscibility is based on the premise that a single $T_{g}$ indicates that a uniform blend domain size comparable to the macromolecular cooperative length or to the macromolecular radius of gyration, i.e., $2 \leq d_{d} \leq 15 \mathrm{~nm}$. This approach has already been discussed in Sect. 2.5.2. It is important to recognize that a single $T_{g}$ is not a measure of miscibility, but rather an indication of the state of dispersion. There are several equations relating $T_{g}$ to composition (Utracki 1989). One approach (Couchman 1978) proposed the following relation for the $T_{g}$ of miscible systems:

$$
\ln T_{g}=\frac{\sum_{i} w_{i} \Delta C_{P i} \ln T_{g i}}{\sum_{i} w_{i} \Delta C_{P i}} \quad\left(\text { with } \Delta C_{P i} \equiv C_{P}^{\text {liquid }}-C_{P}^{\text {glass }} \text { for polymer } i\right)
$$

where $w_{i}$ and $T_{g i}$ are, respectively, the weight fraction and glass transition temperature of polymer $i$ in the blend and $\Delta C_{P i}$ is a difference of the isobaric heat capacity, $C_{P}$, in the liquid and glass states of polymer $i$, assumed to be independent of $T$. From this relationship, several empirical and semiempirical formulas were derived, including the Gordon-Taylor equation, as well as the Fox equation. Note that these relations are valid only for miscible systems. The latter one

$$
\frac{1}{T_{g}}=\sum_{i} \frac{w_{i}}{T_{g i}} \quad\left(\text { or : } \quad \sum_{i} w_{i}\left(1-\frac{T_{g}}{T_{g i}}\right)=0\right)
$$

is particularly simple and ubiquitously used, even applied to calculate blends' composition from measured values of $T_{g}$ (this use should be limited to situations where 
the $T_{g}$ versus composition was "calibrated" and confirmed to follow Eq. 2.79). Comparing Eqs. 2.78 and 2.79, it is obvious that the Fox equation ignores the contributions of $\Delta C_{P i}$. To account for this omission, a different relation can be derived, also for miscible blends, for example, in a two-component system (Lu and Weiss 1991, 1992):

$$
T_{g}=\frac{w_{1} T_{g 1}+k w_{2} T_{g 2}}{w_{1}+k w_{2}}-\frac{\chi_{12} R\left(T_{g 2}-T_{g 1}\right) b w_{1} w_{2}}{\Delta C_{P 1}\left(w_{1}+k w_{2}\right)\left(w_{1}+b w_{2}\right)^{2}}
$$

where $k$ and $b$ are ratios of, respectively, $\Delta C_{P}$ 's and densities of polymers 1 and 2 and $\chi_{12}$ is the binary interaction parameter. Thus, the relation makes it possible to compute the interaction parameter of miscible blends from $T_{g}$ versus composition dependencies.

In a different approach, starting from Eq. 2.78, the following dependence was derived for binary blends (Utracki and Jukes 1984):

$$
w_{1} \ln \left(T_{g} / T_{g 1}\right)+k w_{2} \ln \left(T_{g} / T_{g 2}\right)=0
$$

For a miscible blend, the parameter $k$ is equal to $k=\Delta C_{P 1} / \Delta C_{P 2}$ (relaxing this condition, transforms Eq. 2.81 into a semiempirical one, valid for either miscible or immiscible systems). The dependence should be symmetrical, i.e., it must be valid when the indices are exchanged. Thus, miscibility requires that $k=1 / k=1$. The larger the difference between $k$ and $1 / k$, the larger is the immiscibility of the system. The dependence should not be used for strongly associating polymer blends where blend $T_{g}$ may reach values higher than those observed for either pure component. Such miscible, hydrogen-bonded, or donor-acceptor pairs are well described by a single parameter relation (Utracki 1989):

$$
T_{g}=\left(1+K^{*} w_{1} w_{2}\right)\left[w_{1} T_{g 1}^{3 / 2}+w_{2} T_{g 2}^{3 / 2}\right]^{2 / 3}
$$

where $K^{*}$ is a material parameter, with a value that increases with stronger polymer/ polymer association.

Several methods of $T_{g}$ determination make it possible to measure the width of the glass transition temperature (TW). The value of TW can be more reliable in assessing the degree of miscibility than $T_{g}$. For example, TW of $6{ }^{\circ} \mathrm{C}$ was determined for neat polymers, $\mathrm{TW}=10{ }^{\circ} \mathrm{C}$ for miscible blends, and $\mathrm{TW}=32{ }^{\circ} \mathrm{C}$ for blends approaching immiscibility (Fried et al. 1978). By measuring $T_{g}$ and TW for samples annealed at different temperatures and then quenched, one may be able to determine the level of miscibility and hence construct a simplified phase diagram. This has been done for numerous blends, like those listed in Table 2.26, and others, e.g., for PS/PTMPC, $\mathrm{PVC} /$ poly $(\alpha$-methylstyrene-co-methylmethacrylate-co-acrylonitrile), and NBR/EVAc (Casper and Morbitzer 1977) (vide infra, Table 2.27).

To construct the phase diagram, thin blend specimens should be prepared. The preferred method is to cast film from a common solvent. However, it has been 
Table 2.27 A few examples of $T_{g}$ measurements of polymer blends

\begin{tabular}{|c|c|c|}
\hline Blend & Comment & References \\
\hline PVDC/aliphatic polyesters & $\begin{array}{l}\text { THF cast films; } T_{g} \text { measured by DSC on } \\
\text { samples annealed at } 460 \mathrm{~K}\end{array}$ & 1 \\
\hline $\begin{array}{l}\text { Oligo(styrene-co-allyl alcohol)/ } \\
\text { aliphatic polyesters }\end{array}$ & $\begin{array}{l}\text { Specimens mechanically mixed at } T=T_{g}+ \\
70^{\circ} \mathrm{C} \text {; DSC at } 20^{\circ} \mathrm{C} / \mathrm{min} \text {; UCST directly } \\
\text { observed }\end{array}$ & 2 \\
\hline PS/poly(styrene-co-4-bromostyrene) & $\begin{array}{l}\text { Films cast from } \mathrm{CHCl}_{3} \text { of } \mathrm{CH}_{2} \mathrm{Cl}_{2} ; \mathrm{DSC} \\
(10 \mathrm{mg}) \text { at } 20^{\circ} \mathrm{C} / \mathrm{min} . \mathrm{UCST} \text { reported }\end{array}$ & 3 \\
\hline Poly(aryl ether ketone) blends & $\begin{array}{l}T_{g} \text { linear dependence on mole fraction of } \\
\text { ketone groups }\end{array}$ & 4 \\
\hline $\begin{array}{l}\text { PMMA/SAN/SMA PEM/SAN/SMA } \\
\text { MAN/SAN/SMA }\end{array}$ & $\begin{array}{l}\text { Samples were either cast from MEK or melt } \\
\text { blended; DSC at } 20{ }^{\circ} \mathrm{C} / \mathrm{min} ; T_{g} \text { from the } \\
\text { onset during the second heating cycle }\end{array}$ & 5 \\
\hline 1,2-PB/1,4-PI & $\begin{array}{l}\text { Polymers co-dissolved in benzene, then } \\
\text { freeze-dried; DSC at } 10{ }^{\circ} \mathrm{C} / \mathrm{min}^{\prime} T_{g} \text { and TW } \\
\text { measured in duplicate or triplicate }\end{array}$ & 6 \\
\hline SAN/SMMA/MAN & $\begin{array}{l}\text { Ternary blends prepared in THF, } \\
\text { precipitated by } \mathrm{MeOH} \text {, then dried; DSC at } \\
20^{\circ} \mathrm{C} / \text { min over } T=310-430 \mathrm{~K}\end{array}$ & 7 \\
\hline PC/TMPC/SAN/SMMA & $\begin{array}{l}\text { Samples cast from THF; DSC at } 20^{\circ} \mathrm{C} / \mathrm{min} \text {; } \\
T_{g} \text { taken at onset. Phase diagrams } \\
\text { constructed }\end{array}$ & 8 \\
\hline $\begin{array}{l}\text { SMMA/poly(butyl-co-hexafluoro- } \\
\text { carbonate) }\end{array}$ & $\begin{array}{l}\text { Samples dissolved in } \mathrm{CH}_{2} \mathrm{Cl}_{2} \text {, precipitated } \\
\text { by } \mathrm{MeOH} \text {, and dried. } T_{g} \text { taken at onset } \\
\text { during the second heating }\end{array}$ & 9 \\
\hline $\begin{array}{l}\text { Poly }(\alpha-\text { MSAN }) / S A N, \text { TMPC, PVC, } \\
\text { PPE or PMMA; PMMA-GMA/SAN } \\
\text { or TMPC }\end{array}$ & $\begin{array}{l}\text { Samples either cast from THF, or hot cast } \\
\text { from DMF or acetonitrile, dried at } 150^{\circ} \mathrm{C} \text { for } \\
2 \text { days; DSC at } 20^{\circ} \mathrm{C} / \mathrm{min} \text {. Diverse phase } \\
\text { diagrams }\end{array}$ & 10 \\
\hline PC/poly(ET-co-caprolactone) & $\begin{array}{l}\text { Samples cast from } \mathrm{CHCl}_{3} ; \mathrm{DSC} \text { at } 20^{\circ} \mathrm{C} / \mathrm{min} \\
T \leq 530 \mathrm{~K} . T_{g} \text { taken at half-height }\end{array}$ & 11 \\
\hline PMMA-GMA/PVDF & $\begin{array}{l}\text { Samples cast from DMF; DSC at } 20^{\circ} \mathrm{C} / \mathrm{min} \\
\text { to } 190^{\circ} \mathrm{C} \text {; miscibility only for PMMA-GMA } \\
\text { with GMA }<35.7 \text { wt } \%\end{array}$ & 12 \\
\hline PS/PC or oligo(cyclic-carbonate) & $\begin{array}{l}\text { Samples hot cast from } o \text {-dichlorobenzene; } \\
\text { DSC at } 20^{\circ} \mathrm{C} \text {; samples annealed at } 200{ }^{\circ} \mathrm{C} \\
\text { for } 5 \mathrm{~min}\end{array}$ & 13 \\
\hline PS/PCHMA & $\begin{array}{l}\text { Dissolved in THF, precipitated by } \mathrm{MeOH} \text {; } \\
\text { DSC at } 10^{\circ} \mathrm{C} / \mathrm{min} ; T_{g} \text { taken at midpoint of } \\
\text { inflection }\end{array}$ & 14 \\
\hline PBT/Poly(ester carbonate) & $\begin{array}{l}\text { DSC at } 20^{\circ} \mathrm{C} / \mathrm{min} ; T_{g} \text { from second scan. } \\
\text { Solution cast samples gave two } T_{g} \text { 's; } \\
\text { precipitated from solution or melt mixed } \\
\left(\text { at } 250^{\circ} \mathrm{C} \text { ) systems had only one } T_{g}\right.\end{array}$ & 15 \\
\hline $\mathrm{PEI} / \mathrm{PAr}$ & $\begin{array}{l}\text { Melt mixed at } 300^{\circ} \mathrm{C} \text {; DSC at } 20^{\circ} \mathrm{C} / \mathrm{min} . T_{g} \\
\text { taken at onset }\end{array}$ & 16 \\
\hline CR/PEMA & Melt mixed at $100^{\circ} \mathrm{C}$; DSC at $20^{\circ} \mathrm{C} / \mathrm{min}$ & 17 \\
\hline
\end{tabular}


Table 2.27 (continued)

\begin{tabular}{|c|c|c|}
\hline Blend & Comment & References \\
\hline PMMA/PBMA & $\begin{array}{l}\text { Samples prepared by } \mathrm{MeOH} \text { precipitation of } \\
\text { acetone solutions; DSC }(20 \mathrm{mg}) \text { at } 10^{\circ} \mathrm{C} / \mathrm{min}\end{array}$ & 18 \\
\hline PEEK/PEI & $\begin{array}{l}\text { Melt mixed at } 400^{\circ} \mathrm{C} \text {, quenched in ice water } \\
\text { into } 0.2-0.4 \mathrm{~mm} \text { sheets. DSC }(10 \mathrm{mg}) \text { at } \\
20^{\circ} \mathrm{C} / \mathrm{min} \text {. Miscible blends }\end{array}$ & 19 \\
\hline $\begin{array}{l}\text { Poly(aryl ether ketone)/aromatic } \\
\text { thermoplastic polyimides }\end{array}$ & $\begin{array}{l}\text { Melt mixed at } 400-455^{\circ} \mathrm{C}(\mathrm{ca} .50 \mathrm{mg} \text {, } \\
\left.\text { between two sheets of Kapton }{ }^{\mathrm{TM}}\right) \text {; DSC at } \\
20^{\circ} \mathrm{C} / \mathrm{min}\end{array}$ & 20 \\
\hline $\begin{array}{l}\text { Poly(styrene-co-2,4-dinitrostyrene)/ } \\
\text { PVME or PPE }\end{array}$ & $\begin{array}{l}\text { Samples cast from DMF; DSC at } 20^{\circ} \mathrm{C} / \mathrm{min} \text {. } \\
\text { Phase diagram with LCST found }\end{array}$ & 21 \\
\hline $\begin{array}{l}\mathrm{PVC} \text { or c-PVC/poly(caprolactam- } \\
\text { co-caprolactone) }\end{array}$ & $\begin{array}{l}\text { Samples co-precipitated from } p \text {-xylene, } \\
\text { THF, or DMSO; DSC at } 10^{\circ} \mathrm{C} ; T_{g} \text { from } \\
\text { onset and inflection point }\end{array}$ & 22 \\
\hline Cellulose diacetate/PVP & Samples by solution casting; DSC at $10^{\circ} \mathrm{C}$ & 23 \\
\hline PMMA/PEG/Phenoxy & $\begin{array}{l}\text { Samples melt mixed; DSC at } 20^{\circ} \mathrm{C} / \mathrm{min} \text {. } \\
\text { Immiscibility window found }\end{array}$ & 24 \\
\hline SAN/PAr-co-TMPAr & $\begin{array}{l}\text { Samples } \mathrm{MeOH} \text { precipitated from } \mathrm{CH}_{2} \mathrm{Cl}_{2} \text {; } \\
\text { DSC at } 20^{\circ} \mathrm{C} / \mathrm{min} \text {. Miscibility map given }\end{array}$ & 25 \\
\hline PVC/SMMA & $\begin{array}{l}\text { Samples melt mixed; DSC at } 20^{\circ} \mathrm{C} / \mathrm{min} \\
\text { (contrast enhanced by physical aging, } 46 \mathrm{~h} \text { at } \\
60^{\circ} \mathrm{C} \text { ) }\end{array}$ & 26 \\
\hline
\end{tabular}

References: 1. Aubin et al. 1983; 2. Woo et al. 1984; 3. Strobl et al. 1986; 4. Harris and Robeson 1987; 5. Brannock and Paul 1990; 6. Roovers and Toporowski 1992; 7. Cowie et al. 1992c; 8. Kim and Paul 1992; 9. Takakuwa et al. 1994; 10. Gan et al. 1994; Gan and Paul 1994b; 11. Dezhu et al. 1995; 12. Gan and Paul 1995; 13. Nachlis et al. 1995; 14. Friedrich et al. 1996; 15. Rodriguez et al. 1996; 16. Bastida et al. 1996; 17. Kundu et al. 1996; 18. Sato et al., 1996a, b; 19. Goodwin and Simon 1996; 20. Sauer et al. 1996; 21. Fernandez et al. 1997; 22. van Ekenstein et al. 1997; 23. Jinghua et al. 1997; 24. Hong et al. 1997; 25. Ahn et al. 1997; 26. Dompas et al. 1997

observed that the blend thermograms depend on the type of solvent used for casting the film. For example, PVC/PHMT blends cast from toluene had a single $T_{g}$, whereas when cast from 1,4-dioxane it exhibited two $T_{g}$ 's, a fact that it is not completely unexpected, since small differences in the polymer/solvent $\chi$ results in quite substantial enthalpic contributions in the free energy of mixing, in the order of $\chi N, \mathrm{~N}$ being the size (degree of polymerization) of the polymer, which would cause in a better dissolution of one of the two polymers in any given solvent and, in turn, would result in differences in the miscibility of the cast blend. Clearly, caution is advised: Preferably the procedure should be carried out using specimens prepared by different methods; specimens should be annealed at temperatures located on both sides of the expected spinodal and then quenched. The most popular method for detecting $T_{g}$ is the differential scanning calorimetry (DSC), owing to the simplicity of the experiments and the small specimen sizes required (a few $\mathrm{mg}$ ). Using substantially more complex experiments and analysis, more information can be obtained from a dynamic test: either dielectric relaxation spectroscopy, or dynamic mechanical analysis (shear, bending or tensile, preferably at low and 
constant test frequency). $T_{g}$ can also be determined by dilatometry. The method extends testing of the blend miscibility to higher pressures, as those expected during processing (Jain et al. 1982; Walsh and Zoller 1987; Zoller and Walsh 1995). The pressure effects are not negligible, for example, for PPE/PS system, the pressure gradient of $T_{g}\left(d T_{g} / d P\right)$ was reported to range from 4.3 to $8.2^{\circ} \mathrm{C} / \mathrm{GPa}$, depending on the composition (Zoller and Hoehn 1982).

One shortcoming of the method can be demonstrated by the fact that, even for immiscible blends, rarely two $T_{g}$ 's can be detected for compositions containing less than $20 \mathrm{wt} \%$ of the dispersed phase. Beyond any instrumental detection limits, the experimental range of resolution depends also on the difference between the $T_{g}$ 's of the two polymers $\left(\Delta T_{g}=T_{g 1}-T_{g 2}\right)$. Since the width of the glass transition can be as large as $\mathrm{TW}=40^{\circ} \mathrm{C}$, this method of assessment of miscibility should not be used for systems with $\Delta T_{g} \leq T W / 2 \approx 20^{\circ} \mathrm{C}$. Table 2.27 provides some examples studies of blend miscibility by means of $T_{g}$ measurements; older data can be found in Utracki (1989).

\subsubsection{Spectroscopic Methods: NMR}

For the studies of interactions in polymer blends, nuclear magnetic resonance (NMR) and Fourier transform infrared spectroscopy (FTIR) are of principal significance. The NMR methods for the studies of polymer blends are well described in textbooks as well as in several reviews (Olabisi et al. 1979; Robeson 1980; Martuscelli et al. 1983; Kaplan 1984; McBrierty and Packer 1993; Cheng et al. 2011). The NMR parameters used in the determination of polymer/polymer miscibility are mainly the times related to the half-life of the spin relaxation, such as the spin-lattice relaxation time $\left(T_{1}\right)$, the spin-spin $\left(T_{2}\right)$, and the spin-lattice in the rotating frame $\left(T_{1 \rho}\right)$. The shorter the relaxation time, the broader is the NMR line width (for solid samples the lines are broad, a manifestation of slow reorientation of bonds, whereas for liquids and solution they are narrow, as expected from faster bond reorientation). The position of the lines, i.e., the precessional frequency of the nucleus, depends on its chemical environment, spatial configuration, and interactions. The position of the peak or the so-called chemical shift (usually quoted as $d$ in parts-per-million, ppm) is a reflection of the energetic state of the nucleus, while the line intensity is that of its population. For example, $T_{1 \rho}$ was used to analyze interactions between PVC and polymethacrylates: poly(cyclohexyl methacrylate) (PCHMA), poly(phenyl methacrylate) (PPMA), and poly(benzyl methacrylate) (PBzMA) (Sankarapandian and Kishore 1996). The NMR-detected domain size was between 3 and $15 \mathrm{~nm}$. The miscibility of the same systems was also analyzed by measuring $T_{g}$. Both methods indicated miscibility for the PVC/PCHMA blends and immiscibility for the PVC/PBzMA system; however, for the PVC/PPMA 1:1 blends, while $T_{g}$ indicated miscibility, the $T_{1 \rho}$ NMR data showed that the same system is immiscible.

Modern solid-state NMR involves the use of very short radio-frequency pulses (of variable duration from 1 to $200 \mathrm{~ms}$ ) and can be complemented with real-time Fourier transform analysis and multiple scan capability. Standard NMR enhancements nowadays, such as scalar (low power, ca. $4 \mathrm{kHz}$ ) and dipolar (about $45 \mathrm{kHz}$ ) decoupling, magic angle spinning, spectra of multiple elemental isotopes beyond 
Fig. 2.26 An example of NMR used to probe local environment of a polymer in a blend. ${ }^{15} \mathrm{~N}$ CPMAS (crosspolarization, magic angle spinning) NMR spectra of polyamide- 6 in a blend with polyketone. PA-6 in a PK/PA 6:4 blend (a) shows primarily (70\%) its $\alpha$-crystal phase, whereas in its pure form (b) PA-6 shows a $60 \% \gamma$ and $40 \% \alpha$ crystal (Data from Asano, Chap. 5 in Cheng et al. 2011)
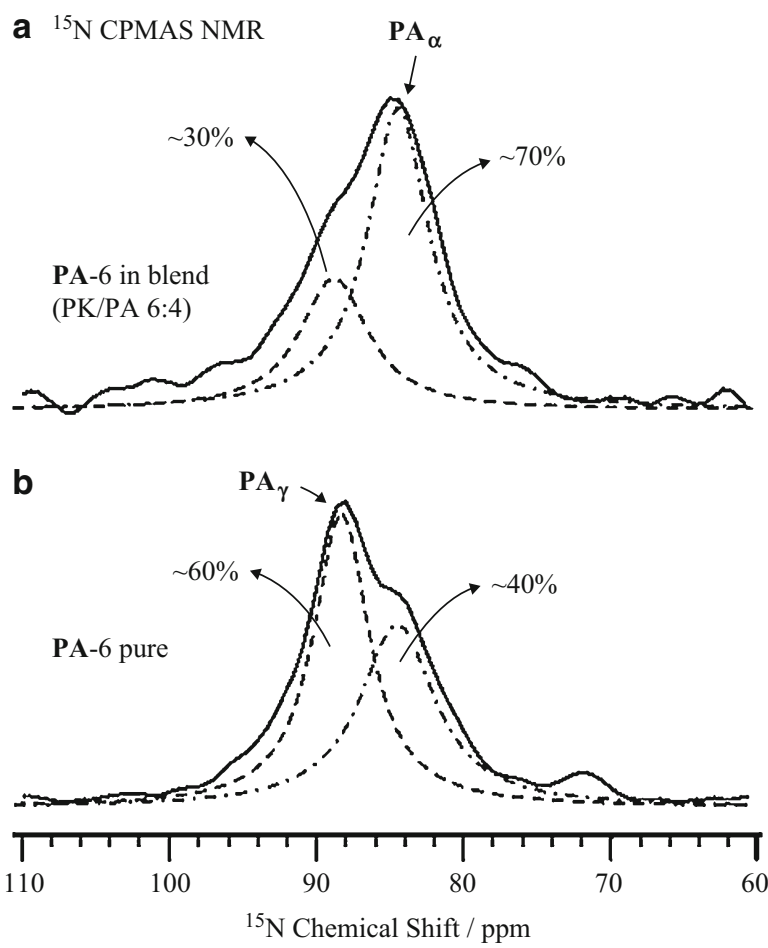

${ }^{1} \mathrm{H}$ and ${ }^{13} \mathrm{C}$, multi-pulse (multidimensional NMR), and cross-polarization methods, enable spectra for solid samples with resolutions similar to those known for liquids (Webb 2006; White and Wachowicz 2008). Such spectra provide precise information on the local environment of selected nuclei, configuration, interactions, and sample morphology (Fig. 2.26).

The interactions between PVDF with PMMA, PVAc, or PVME were studied by dipolar-dipolar decoupling, cross-polarization, magic angle spinning, and highresolution ${ }^{13} \mathrm{C}$ NMR (Lin 1983; Ward and Lin 1984). Clear peak assignment for each carbon was made, and peak intensities were measured. It was found that blending has little effect on the peak frequency but a significant effect on its intensity. For example, the attenuation $\left(A_{t}\right.$ as a $\%$ of the observed to the expected intensities) varied with the method of blend preparation. For PVDF/PMMA 1:1 blends, when cast from DMF $A_{t}$ was $100 \%$ (immiscible), while when cast from MEK $A_{t}$ was $60-75 \%$, and for extruded blends $A_{t}$ was $26-49 \%$ (miscible). Much clearer differences were obtained in PMAA/PVAc blends studied by ${ }^{13} \mathrm{C}$ CPMAS (cross-polarization, magic angle spinning) NMR (Fig. 2.27).

Information on short-distance spatial proximity between different segments of molecules can be obtained using the proton spin-diffusion NMR method. This is a particularly valuable method for the characterization of polymer blends. For example, in case of PS/PVME cast films, the method provided information on blend composition, fraction of interacting groups (phenyl from PS with ether from 

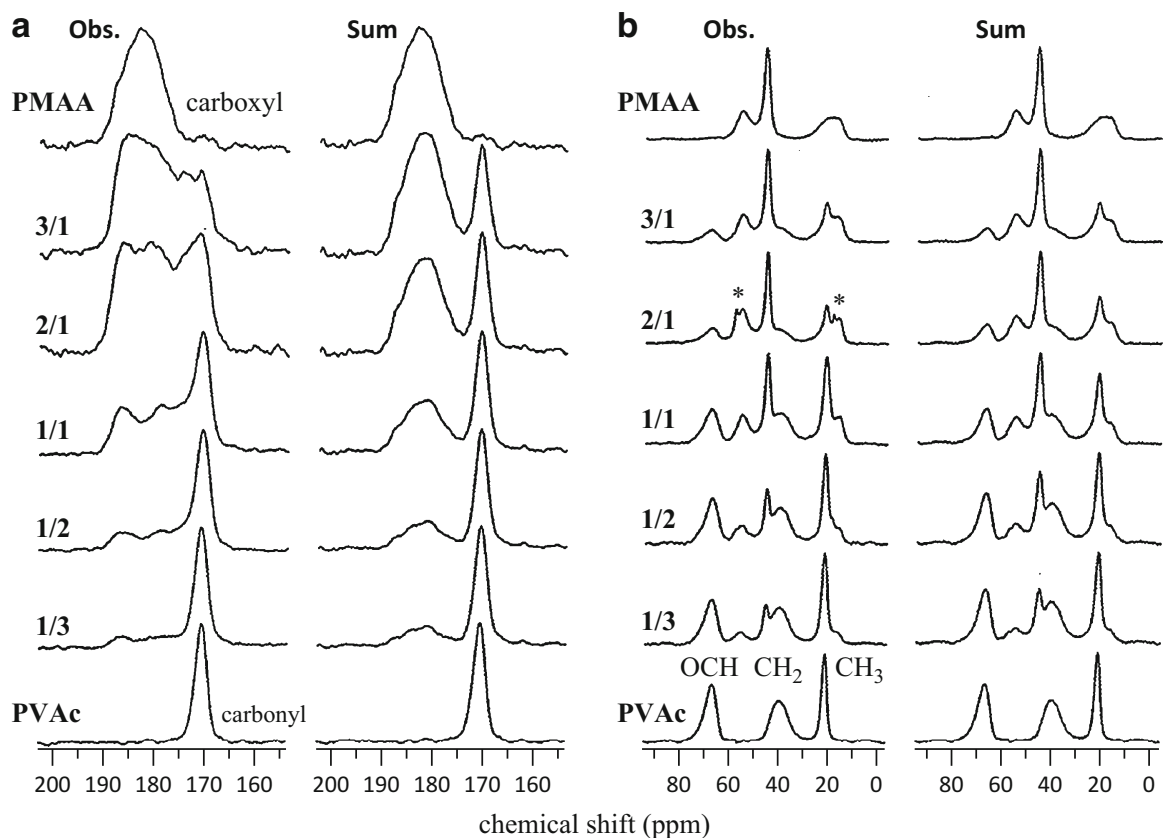

Fig. $2.27{ }^{13} \mathrm{C}$ CPMAS NMR spectra of PMAA (top line), PVAc (bottom line), and several PMAA/PVAc blends. (a) carboxyl regions of PMAA and carbonyl regions of PVAc; (b) aliphatic regions. The weighted sums (of the pure PMAA and pure PVAc ${ }^{13} \mathrm{C}$ NMR spectra) are also depicted on the right of the corresponding observed spectra (left columns). The blend formation results in strong qualitative changes in the $\mathrm{OC}=\mathrm{O}$ carbon, but not so much in the carbons of the aliphatic region (Data from Asano et al. 2002)

PVME), and group mobility within each of the three domains (PS, PVME, and PS-PVME). Again, it was found that different degree of dispersion is obtained when casting films from different solvents (Caravatti et al. 1985; 1986).

Miscibility of PEEKK/PEI 1:1 blend was analyzed using solid-state NMR (Schmidt-Rohr et al. 1990). This work involved tagging of ${ }^{1} \mathrm{H}$ magnetization, based on chemical shift difference, ${ }^{1} \mathrm{H}$ spin diffusion for controlled mixing times (tuned to probe $0.5-50 \mathrm{~nm}$ length scales), and high-resolution ${ }^{13} \mathrm{C}$ detection. The sample was prepared by compounding at $653 \mathrm{~K}$. Intimate mixing on a molecular scale was concluded. An extension of this method employed $2 \mathrm{D}{ }^{13} \mathrm{C}-{ }^{13} \mathrm{C}$ CPMAS NMR combined with multiple alternating depolarization (MAD) ${ }^{13} \mathrm{C}(\mathrm{HH}){ }^{13} \mathrm{C}$ pulses, to probe PS/PXE blends (Hou et al. 2004); albeit tedious and lengthy, this method yielded substantially improved sensitivity in unlabeled samples and much better contrast between blend components compared to ${ }^{1} \mathrm{H}$ spin-diffusion NMR.

Two-dimensional ${ }^{2} \mathrm{H}$ NMR was used to analyze miscibility in blends of poly1,4-polyisoprene with polyvinylethylene (PI/PVE) (Arendt et al. 1994; Chung et al. 1994). The blends were prepared by casting $3 \mathrm{wt} \%$ toluene solution. The rate of reorientation as a function of temperature near $T_{g}$ was determined for both components. It was found that the system is miscible, but the glass transition is 
broad owing to the wide distribution of segmental motions arising from the differences in the rates of the two polymers. As a result, the PIP/PVE blends were found to be rheologically complex: In spite of miscibility, the timetemperature superposition was found to be invalid.

High-resolution ${ }^{13} \mathrm{C}$ NMR spectroscopy was used to analyze miscibility of POM with terphenol $\left(M_{w}=600 \mathrm{~g} / \mathrm{mol}\right)$. The size of the heterogeneity in the amorphous phase was estimated as $1 \mathrm{~nm}$. The ${ }^{1} \mathrm{H}$ spin-diffusion analysis indicated a homogenous mixing on the molecular level (Egawa et al. 1996).

\subsubsection{Spectroscopic Methods: Infrared}

The use of infrared spectroscopy for the characterization of polymer blends is extensive (Olabisi et al. 1979; Coleman and Painter 1984; Utracki 1989; He et al. 2004 and references therein; Coleman et al. 1991, 2006). The applicability, fundamental aspects, as well as principles of experimentation using infrared dispersive double-beam spectrophotometer (IR) or computerized Fourier transform interferometers (FTIR) were well described (e.g., Klopffer 1984).

FTIR has been extensively used to study hydrogen bonding in polymer blends (Ting 1980; Cangelosi 1982; Moskala 1984; Pennacchia 1986; He et al. 2004; Coleman et al. 1991, 2006). These interactions affect not only the $-\mathrm{OH}$ absorption region $\left(3,500-3,600 \mathrm{~cm}^{-1}\right)$, but also the $=\mathrm{CO}$ stretching $\left(1,737 \mathrm{~cm}^{-1}\right)$, the $-\mathrm{CH}_{2}$ symmetric stretching $\left(2,886 \mathrm{~cm}^{-1}\right)$, as well as the fingerprint frequency region $\left(1,300-650 \mathrm{~cm}^{-1}\right)$. As discussed in Sect. 2.6.2.3, FTIR has been used to calculate the strong interaction term of the free energy of mixing, $\Delta G_{H}$ (see Eqs. $2.35 \mathrm{~b}$ and 2.58) (Painter et al. 1988, 2006). The combination of FTIR spectra, which can yield a miscibility map for specific systems, with the fitted model parameters, which can give "theoretical" phase boundaries, can be combined to construct phase diagrams for specific polymer blend systems (Fig. 2.28).

FTIR was also used to analyze the mechanism of interactions in blends of aliphatic polyesters with chlorinated polymers, viz., PVC, PVDC, or PVC-DC. In miscible blends, the polyesters' carbonyl stretching absorption frequency $\left(1,700-1,775 \mathrm{~cm}^{-1}\right)$ was shifted - the shift was absent in immiscible systems - revealing hydrogen bonding between $\mathrm{C}-\mathrm{O}$ and $\alpha$ - or $\beta$-hydrogen. However, there are indications that the interaction mechanism does vary from system to system, e.g., the dipolar $\mathrm{C}=\mathrm{O}$ with $\mathrm{C}-\mathrm{Cl}$ interactions have been also identified (Coleman et al. 1983; Prud'homme 1982; Garton et al. 1983; Morra and Stein 1984; Albert et al. 1986). A typical approach to construct a miscibility map from FTIR data is shown in Fig. 2.28, and a short summary of FTIR studies of polymer blends in Table 2.28.

There are publications on the use of other spectroscopic techniques, such as Brillouin scattering, photoacoustic, and Raman spectroscopy. The primary application of these has been to study the heterogeneities in polymer blends, viz., crystallization or phase separation.

\subsubsection{Microscopy}

Microscopy methods, in the broader sense of methods that provide direct morphology imaging, can be divided into several categories: optical microscopy (OM), 

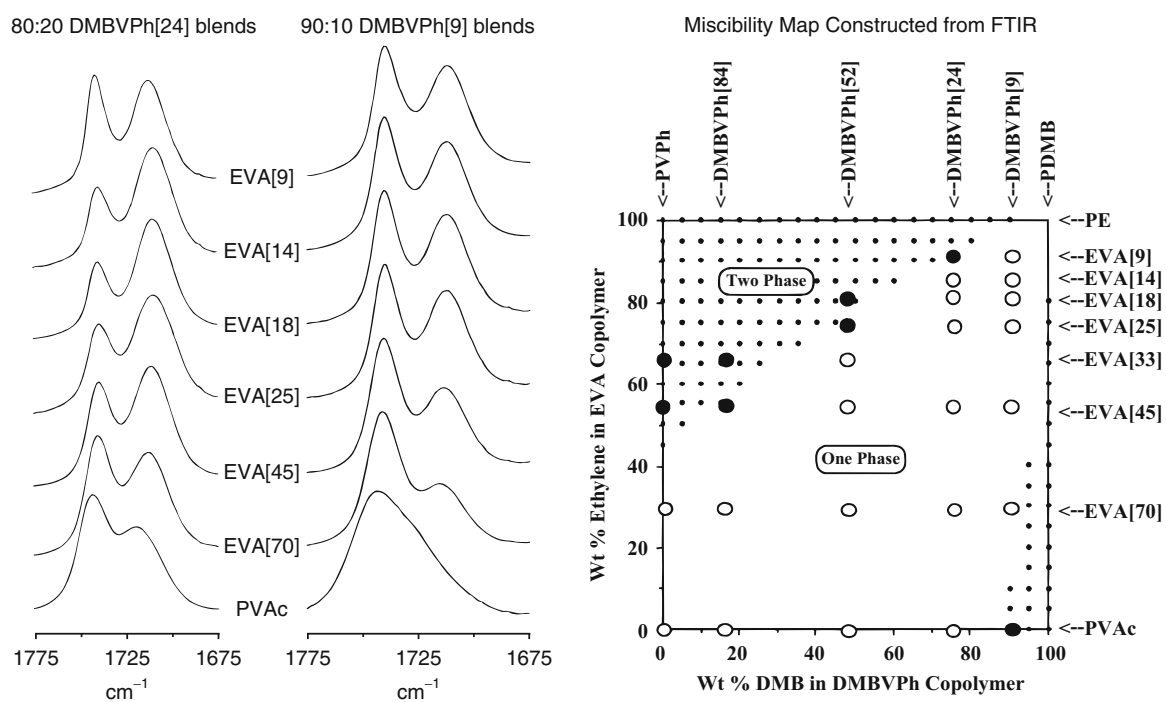

Fig. 2.28 IR spectra in the carbonyl stretching region for blends of 2,3-dimethylbutadiene-co-4vinylphenol (DMBVPh) with PVAc, EVA[70], EVA[45], EVA[25], EVA[18], EVA[14], and EVA[9] recorded at $100{ }^{\circ} \mathrm{C}$; numbers in brackets indicate the \% of the comonomer, $\mathrm{Ph}$ or VA, in each polymer. (Left) IR spectra of 80:20 wt\% DMBVPh-[24] blends; (Middle) IR spectra of 90:10 wt\% DMBVPh-[9] blends; (Right) Miscibility map calculated at $100{ }^{\circ} \mathrm{C}$ for DMBVPh/EVA blends: areas encompassed by small black dots denote the predicted two-phase regions; experimentally determined single- and two-phase blends are denoted by the open and filled large circles, respectively (Data from Pehlert et al. (1997)

scanning electron microscopy (SEM), transmission electron microscopy (TEM), atomic force microscopy (AFM), and several modifications of these techniques. For example, the scanning transmission electron microscopy (STEM) and low-voltage scanning electron microscopy (LVSEM, at $0.1-2 \mathrm{kV}$ accelerating voltage) are particularly useful for polymer blends (Vesely and Finch 1988). STEM uses ultrathin stained films, providing images with a few nanometers resolution. LVSEM provides about tenfold increased image contrast (in comparison to the conventional SEM) with almost no charging problem (Berry 1988). Owing to shallow sampling depth and low energy of the secondary electrons, conductive coating is not needed. The method uses flat, microtomed specimens providing image quality comparable to that of TEM (Vesely 1996; a nice review of microscopy methods for blends). In all cases, microscopy is considered a necessary second method of characterization for polymer blends, since it provides the required morphological information needed to explain results from spectroscopy, $T_{g}$, or other measurements.

In most cases, some mode of sample "preparation" has to be used after the blend formation, viz., staining, swelling, fracturing, or etching. These are very appropriate for and have been extensively used to characterize morphology of immiscible blends, but they have obvious severe shortcomings in miscible or partially miscible 
Table 2.28 A few examples of FTIR measurements of polymer blends

\begin{tabular}{|c|c|c|}
\hline Blend & Comment & References \\
\hline PVDF/PMMA & $\begin{array}{l}\text { Blending slightly affected the carbonyl stretching near } \\
1,735 \mathrm{~cm}^{-1}\end{array}$ & 1 \\
\hline $\mathrm{PPE} / \mathrm{PS}$ & $\begin{array}{l}\text { FTIR peak ratio } 1,030 / 700 \mathrm{~cm}^{-1} \text { used for the determination } \\
\text { of composition }\end{array}$ & 2 \\
\hline $\mathrm{PPE} / \mathrm{PS}$ & Strongest interactions for $30 \mathrm{wt} \% \mathrm{PPE}$ & 3 \\
\hline $\mathrm{PPE} / \mathrm{PS}$ & $\begin{array}{l}\text { FTIR was used for characterizing macromolecular } \\
\text { orientation in solid state }\end{array}$ & 4 \\
\hline $\mathrm{PC} / \mathrm{PCL}$ & Miscibility in the amorphous phase & 5 \\
\hline $\mathrm{PC} / \mathrm{PBT}$ & Miscibility in the amorphous phase & 6 \\
\hline PCL/Phenoxy & $\begin{array}{l}\text { H-bonding between the }-\mathrm{OH} \text { group of phenoxy and } \mathrm{C}=\mathrm{O} \text { of } \\
\text { PCL }\end{array}$ & 7 \\
\hline PS/PVME & $\begin{array}{l}\text { Changes in molecular environment were easiest detected } \\
\text { using the vibrations: in PS the out-of-plane } \mathrm{CH} \text {, and in } \\
\text { PVME the } \mathrm{COCH}_{3} \text { at } 700 \text { and } 1,100 \mathrm{~cm}^{-1}\end{array}$ & 8 \\
\hline $\begin{array}{l}\text { PF (Novolac)/PS, } \\
\text { SAN, PEA, PVAc, } \\
\text { PEMA, PMMA, } \\
\text { PMPS, PC, or PVME }\end{array}$ & $\begin{array}{l}\text { Novolac (PF) blends: frequency shifts in CO vibration from } \\
1,774 \text { to } 1,752 \mathrm{~cm}^{-1} \text { due to hydrogen bonding in miscible } \\
\text { blends }\end{array}$ & 9 \\
\hline $\mathrm{PET} / \mathrm{PC}$ & FTIR used to study transesterification & 10 \\
\hline PET/PA-6 & Studies of ester-amide exchange reactions & 11 \\
\hline PU/poly(EG-co-PG) & $\begin{array}{l}\text { The N-H stretching }\left(3,500-3,100 \mathrm{~cm}^{-1}\right) \text { was found sensitive } \\
\text { to interactions }\end{array}$ & 12 \\
\hline $\begin{array}{l}\text { PVPh/PVAc, EVAc, } \\
\text { PCL, PPL, PMA, PEA, } \\
\text { PBA, or P2EHA }\end{array}$ & $\begin{array}{l}\text { Poly(4-vinyl phenol) blends: the amount of free and bonded } \\
\mathrm{C}=\mathrm{O} \text { vibrations were determined fort the } \mathrm{PVPh} / \text { hydrogen- } \\
\text { bonding polymer blends, using a curve fitting procedure }\end{array}$ & 13 \\
\hline $\begin{array}{l}\text { poly(DMB-co-VPh)/ } \\
\text { EVA }\end{array}$ & $\begin{array}{l}\text { 2,3-dimethylbutadiene-co-4-vinylphenol (DMBVPh) } \\
\text { blends with EVA (VA comonomer: } 0 \% \text { to } 100 \% \text { ). } \\
\text { Miscibility map constructed (see also Fig. } 2.28 \text { ) }\end{array}$ & 14 \\
\hline $\begin{array}{l}\text { poly(S-co-VPh)/ } \\
\text { PBMA, PHMA, or } \\
\text { PTHF }\end{array}$ & $\begin{array}{l}\text { Poly(styrene-co-4-vinyl phenol) blends: the hydroxyl } \\
\text { stretching }\left(3,100-3,700 \mathrm{~cm}^{-1}\right) \text { and "fingerprint" } \\
\left(600-1,650 \mathrm{~cm}^{-1}\right) \text { regions were analyzed. The bands } 3,525 \\
\text { and } 3,100-3,500 \mathrm{~cm}^{-1} \text { were assigned, respectively, to free } \\
\text { and hydrogen-bonded structures }\end{array}$ & 15 \\
\hline PVC/SAN & $\begin{array}{l}\text { Nitrile stretch vibration region }\left(2,260-2,220 \mathrm{~cm}^{-1}\right) \text { and } \\
\mathrm{C}-\mathrm{Cl} \text { absorption }\left(660-580 \mathrm{~cm}^{-1}\right) \text { were used to characterize } \\
\text { the interactions }\end{array}$ & 16 \\
\hline PPE/PS & $\begin{array}{l}\text { New method of IR-data treatment was proposed. Weight } \\
\text { fraction of polymer- } 1 \text { in the blend: } x_{1}=a_{o}+a_{1} R /\left(1+a_{2} R\right) \text {, } \\
\text { where } a_{i} \text { are parameters, and } R=A_{1} /\left(A_{1}+A_{2}\right) \text { is the } \\
\text { absorbency fraction }\end{array}$ & 17 \\
\hline $\mathrm{PVPh} / \mathrm{PMMA}$ & $\begin{array}{l}\text { Measurements of solvent cast films showed the H-bonding } \\
\text { extent depended on solvent }\end{array}$ & 18 \\
\hline
\end{tabular}

References: 1. Saito et al. 1987; 2. Mukherji et al. 1980; 3. Koenig and Tovar-Rodriguez 1981; 4. Wang and Porter 1983; 5. Coleman et al. 1984; 6. Birley and Chen 1984; 7. Garton 1983, 1984; 8. Garcia 1987; 9. Coleman and Varnell 1982; Fahrenholtz 1982; 10. Huang and Wang 1986; 11. Pillon and Utracki 1986; 12. Coleman et al. 1988; 13. Coleman et al. 1989; 14. Pehlert et al. 1997; 15. Xu et al. 1991; Serman et al. 1991; 16. Kim et al. 1996; 17. Cole et al. 1996; 18. Dong and Ozaki 1997 
blends. There are numerous factors that reduce resolution in blends, well above instrumental capabilities, and several sources for introduction of artifacts in electron microscopy, e.g., metallization in SEM and $\mathrm{OsO}_{4}$ staining in TEM, introduce an artificial grain structure, especially under greater magnifications. However, even at the highest resolution, it is difficult to obtain sufficient confidence to declare whether the blend is thermodynamically miscible. One of the better sources of this information comes from studies of the diffusion rate of one polymer into another using any of the previously mentioned techniques. Preparation of samples for the observation under TEM is more tedious and exacting than that used for SEM. The specimens have to be hardened and microtomed into ca. $200 \mathrm{~nm}$ thin slices, and most often stained with $\mathrm{Br}_{2}, \mathrm{OsO}_{4}$, or $\mathrm{RuO}_{4}$. Frequently, the SEM and TEM methods are being used in parallel (Karger-Kocsis and Kiss 1987; Kyotani and Kanetsuna 1987; Hsu and Geil 1987; Vesely 1996).

For example, the effects of AN content on miscibility of SAN with PMMA were studied by measuring the thickness of the interphase (Higashida et al. 1995). The effects of concentration, compatibilization, and annealing for PA with either PS or PE (compatibilized by $5 \mathrm{wt} \%$ of PP-MAh or SMA) were studied by SEM (Chen et al. 1988). Compatibilization reduced the diameter of dispersed phases by a factor of ten and stabilized the system against coalescence at the annealing temperature $\left(T=200-230{ }^{\circ} \mathrm{C}\right.$, for at least $\left.1.5 \mathrm{~h}\right)$.

Interesting studies of phase coarsening in PMMA/SMMA blends were followed using interference contrast light microscopy and/or TEM (Andradi and Hellmann 1993). Films, cast from toluene, were homogenized at low temperature and then brought to the spinodal region for phase separation and coarsening; owing to the difference in the refractive indices, good contrast was obtained without etching. The kinetics of phase coarsening in blends of PS with poly(sila- $\alpha$-methylstyrene) was followed under an optical microscope (Maier et al. 1996). The blends have UCST that depends on $M_{w}$. Annealing within the single-phase region, and then jumping to the spinodal region, causes SD and phase coarsening. Similarly, optical and SEM methods were used to study phase separation in blends of PP with isotactic poly(1-butene) (Cham et al. 1994); this system was found to have an UCST.

Reactive compatibilization of PA/SAN blends was followed with careful TEM (Mujumdar et al. 1994a, b). Better contrast was obtained using phosphotungstic acid than $\mathrm{RuO}_{4}$. The binary interaction parameter, $\chi_{12}$, was calculated from the micelle spacing in microphase-separated PS- $b$-PVP system (Clarke et al. 1997). The spacing was determined using an AFM. Details of the polymer blends' morphology and the methods of its characterization were discussed in $>$ Chap. 8 , "Morphology of Polymer Blends" in the first edition of this handbook.

Finally, a creative approach employing optical microscopy involved highthroughput (combinatorial) methods: Here, a single sample was made with a gradient of blend composition in one direction and a linear change in temperature in the normal direction. After sufficient annealing of the samples, the LCST phase diagram can be directly observed with optical microscopy (Meredith et al. 2000, 2002; Karim et al. 2002; Yurekli et al. 2004); see also Fig. 2.29. 
Fig. 2.29 Optical microscopy photograph of a PS and poly(vinyl methyl ether) (PS/PVME) $T-\phi$ combinatorial sample (library) after $16 \mathrm{~h}$ of annealing, showing the lower critical solution temperature (LCST) cloud-point curve. White points are conventional light-scattering cloud points measured independently on separate uniform samples (Meredith et al. 2000)

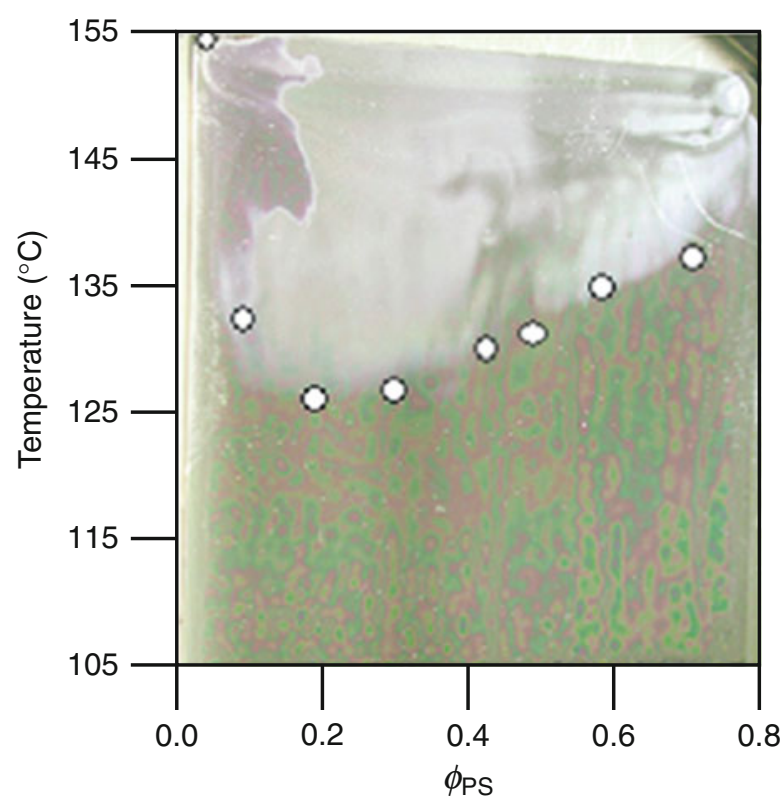

\subsection{Summary and Conclusions}

The information provided in this chapter can be divided into four parts: 1. introduction, 2. thermodynamic theories of polymer blends, 3. characteristic thermodynamic parameters for polymer blends, and 4. experimental methods. The introduction presents the basic principles of the classical equilibrium thermodynamics, describes behavior of the single-component materials, and then focuses on the two-component systems: solutions and polymer blends. The main focus of the second part is on the theories (and experimental parameters related to them) for the thermodynamic behavior of polymer blends. Several theoretical approaches are presented, starting with the classical Flory-Huggins lattice theory and, those evolving from it, solubility parameter and analog calorimetry approaches. Also, equation of state (EoS) types of theories were summarized. Finally, descriptions based on the atomistic considerations, in particular the polymer reference interaction site model (PRISM), were briefly outlined.

As the volume of tabulated values of thermodynamic parameters indicates, the largest pool of data is based on Flory-Huggins type of relations. This is only to be expected since the theory, and the related concept of the binary interaction parameters, either $B$ or $\chi_{12}$, was introduced to polymer science very early, more than a half century ago, in 1941 to be precise. Even with its rather limited applicability, due to its strict assumptions, and the well-recognized complexity of functional dependence [viz., $\chi=\operatorname{chi}\left(T, P, \phi, M_{w}\right.$, MWD, molecular 
structure, stresses, ....), needed for most practical applications] the FH model remains to broadest used and continually developed theoretical framework for blend thermodynamics.

In hindsight, due to its ability to address high $T$ and $P$, the EoS approach has been used with growing frequency to describe the thermodynamic behavior of multicomponent polymer-based systems. Such problems as the temperature- and pressure-dependent miscibility of low molecular weight liquids or polymeric liquids in selected polymers have been successfully solved. For binary blends, the EoS uses two parameters, the first related to the change of specific volume and the other to the energetic interactions. It has been shown that for many cases the values of these experimental constants can be well predicted using the algebraic and geometric means, respectively. Since during polymer processing pressure plays a major role, the EoS is potentially a very valuable tool for polymer researchers and engineers.

Biography The second edition of this chapter was written after Les Utracki had passed away. In an effort to maintain the look and feel of the chapter's first edition, we retained the structure of the original chapter and only updated the content to reflect developments within the last decade. All shortcomings and concerns regarding this chapter should be addressed to manias@psu.edu.

EM would like to dedicate this chapter to the memory of Les Utracki, as well as to my Ph.D. advisors Gerrit ten Brinke and Georges Hadziioannou; all three of these exceptional scientists have seminal and pioneering accomplishments in the field of polymer blends, as reflected in the contents of this chapter, and made invaluable contributions to the first author's education in thermodynamics and blends.

\subsection{Cross-References}

- Crystallization, Micro- and Nano-structure, and Melting Behavior of Polymer Blends

- Interphase and Compatibilization by Addition of a Compatibilizer

- Mechanical Properties of Polymer Blends

- Morphology of Polymer Blends

- Polymer Blends Containing "Nanoparticles"

- Properties and Performance of Polymer Blends

$\checkmark$ Recycling Polymer Blends

\section{Notations and Abbreviations}

Most of the abbreviations used in this chapter are listed in Appendix 1. Pertinent ones are listed under Tables 2.11 and 2.12. 


\section{References}

J.-H. Ahn, C.-K. Kang, W.-C. Zin, Eur. Polym. J 33, 1113 (1997a)

T.O. Ahn, U. Nam, M. Lee, H.M. Feong, Polymer 38, 577 (1997b)

R.G. Alamo, W.W. Graessley, R. Krishnamoorti, D.J. Lohse, J.D. Lonono, L. Mandelkern, F.C. Stehling, G.D. Wignall, Macromolecules 30, 561 (1997)

B. Albert, R. Jerome, P. Teyssie, B. Baeyens-Volant, J. Polym. Sci. Polym. Chem. Ed. 24, 551, 2577 (1986)

R.A. Alberty, Pure Appl. Chem. 73, 1349 (2001)

S.H. Anastasiadis, I. Gancarz, J.T. Koberstein, Macromolecules 21, 2980 (1988)

S.H. Anastasiadis, Interfacial tension in binary polymer blends and the effects of copolymers as emulsifying agents, in Polymer Thermodynamics. Advances in Polymer Science, vol 238 (2011), p. 179

L.N. Andradi, G.P. Hellmann, Polymer 34, 925 (1993)

A. Arbe, A. Alegria, J. Colmenero, S. Hoffmann, L. Willner, D. Richter, Macromolecules 32, 7572 (1999)

B.H. Arendt, R.M. Kannan, M. Zewail, J.A. Kornfield, S.D. Smith, Rheol. Acta. 33, 322 (1994)

A. Asano, M. Eguchi, M. Shimizu, T. Kurotsu, Macromolecules 35, 8819 (2002)

M. Aubin, Y. Bdard, M.-F. Morrissette, R.E. Prud'homme, J. Polym. Sci. Polym. Phys. Ed. 21, 233 (1983)

H.E. Bair, P.C. Warren, J. Macromol. Sci. Phys. B20, 381 (1980)

N.P. Balsara, L.J. Fetters, N. Hadjichristidis, D.J. Lohse, C.C. Han, W.W. Graessley, R. Krishnamoorti, Macromolecules 25, 6137 (1992)

N.P. Balsara, D.J. Lohse, W.W. Graessley, R. Krishnamoorti, J. Chem. Phys. 100, 3894 (1994)

S. Bastida, J.I. Eugizabal, J. Nazabal, Polymer 37, 2317 (1997)

F.S. Bates, G.D. Wignall, Macromolecules 19, 932 (1986)

F.S. Bates, P. Wilzinius, J. Chem. Phys. 91, 3258 (1989)

F.S. Bates, G.D. Wignall, W.C. Koechler, Phys. Rev. Lett. 55, 2425 (1985)

F.S. Bates, S.B. Dierker, G.D. Wignall, Macromolecules 19, 1938 (1986)

V.K. Berry, Scanning 10, 19 (1988)

N.A.M. Besseling, J.M.H.M. Scheutjens, J. Phys. Chem. 98, 11597 (1994)

D.E. Bhagwagar, P.C. Painter, M.M. Coleman, Macromolecules 27, 7139 (1994)

U.R. Bidkar, I.C. Sanchez, Macromolecules 28, 3963 (1995)

A.W. Birley, X.Y. Chen, Brit. Polym. J. 16, 77 (1984)

J. Biros, L. Zeman, D.D. Patterson, Macromolecules 4, 30 (1971)

M. Bohdanecky, J. Kovar, Viscosity of Polymer Solutions (Elsevier, Amsterdam, 1982)

R.F. Boyer, J. Polym. Sci. Part C 14, 267 (1966)

J. Brandrup, E.H. Immergut (eds.), Polymer Handbook, 3rd edn. (Wiley, New York, 1989)

G.R. Brannock, D.R. Paul, Macromolecules 23, 5240 (1990)

G.R. Brannock, J.W. Barlow, D.R. Paul, J. Polym. Sci. Part B Polym. Phys. Ed. 29, 413 (1991)

D. Broseta, G.H. Frederickson, E. Helfand, L. Leibler, Macromolecules 23, 132 (1990)

S.F. Bush, J.M. Methven, D.R. Blackburn, High Perform. Polym. 8, 67 (1996)

T.A. Callaghan, D.R. Paul, Macromolecules 26, 2439 (1993)

T.A. Callaghan, D.R. Paul, J. Polym. Sci. Part B Polym. Phys. 32, 1813 (1994a)

T.A. Callaghan, D.R. Paul, J. Polym. Sci. Part B Polym. Phys. 32, 1847 (1994b)

F. Cangelosi, Ph.D. thesis, University of Connecticut, Storrs, 1982

M.J.R. Cantow, Polymer Fractionation (Academic, New York, 1967)

P. Caravatti, P. Neuenschwander, R.R. Ernst, Macromolecules 18, 119 (1985)

P. Caravatti, P. Neuenschwander, R.R. Ernst, Macromolecules 19, 1889 (1986)

P.M. Cham, T.H. Lee, H. Marand, Macromolecules 27, 4263 (1994)

D. Chandler, H.C. Andersen, J. Chem. Phys. 57, 1930 (1972)

K.K. Chee, Europ. Polym. J. 26, 423 (1990)

C.C. Chen, E. Fontan, K. Min, J.L. White, Polym. Eng. Sci. 28, 69 (1988) 
H.N. Cheng, T. Asakura, A.D. English (eds.), NMR Spectroscopy of Polymers: Innovative Strategies for Complex Macromolecules. ACS Symposium Series, vol. 1077 (Oxford University Press, New York, 2011)

J.S. Chiou, D.R. Paul, J. Appl. Polym. Sci. 42, 279 (1991)

J. Cho, I.C. Sanchez, in Polymer Handbook, ed. by J. Brandrup, E.H. Immergut, E.A. Grulke, 4th edn. (Wiley, New York, 1999)

S. Choi, X. Liu, R.M. Briber, J. Polym. Sci. B Polym. Phys. 36, 1 (1998)

S. Choi, X. Liu, R.M. Briber, Macromolecules 33, 6495 (2000)

G.-C. Chung, J.A. Kornfield, S.D. Smith, Macromolecules 27, 964, 5729 (1994)

C.J. Clarke, A. Eisenberg, J. La Scala, M.H. Rafailovich, J. Sokolov, Z. Li, S. Qu, D. Nguyen, S.A. Schwarz, Y. Strzhemechny, B.B. Sauer, Macromolecules 30, 4184 (1997)

K.C. Cole, Y. Thomas, E. Pellerin, M.M. Dumoulin, R.M. Paroli, Appl. Spectrosc. 50, 774 (1996)

M.M. Coleman, P.C. Painter, Appl. Spectrosc. Rev. 20, 255 (1984)

M.M. Coleman, P.C. Painter, Prog. Polym. Sci. 20, 1 (1995)

M.M. Coleman, P.C. Painter, Miscible Polymer Blends: Background and Guide for Calculations and Design (DEStech Publications, Lancaster, 2006a)

M.M. Coleman, P.C. Painter, Aust. J. Chem. 59, 499 (2006b)

M.M. Coleman, D.F. Varnell, Macromolecules 15, 59 (1983)

M.M. Coleman, D.F. Varnell, J.P. Runt, Polym. Sci. Technol. 20, 937 (1982)

M.M. Coleman, D.F. Varnell, J.P. Runt, Contemp. Topics Polym. Sci. 4, 807 (1984)

M.M. Coleman, D.J. Skrovanek, J. Hu, P.C. Painter, Macromolecules 21, 59 (1988)

M.M. Coleman, A.M. Lichkus, P.C. Painter, Macromolecules 22, 586 (1989)

M.M. Coleman, C.J. Serman, D.E. Bhagwar, P.C. Painter, Polymer 31, 1187 (1990)

M.M. Coleman, J.T. Graf, P.C. Painter, Specific Interactions and the Miscibility of Polymer Blends (Technomic Publishing, Lancaster, 1991)

P.R. Couchman, Macromolecules 11, 1156 (1978)

P.R. Couchman, Phys. Lett. A 70, 155 (1979a)

P.R. Couchman, J. Appl. Phys. 50, 6043 (1979b)

J.M.G. Cowie, J.H. Harris, Polymer 33, 4592 (1992)

J.M.G. Cowie, in Encyclopedia of Polymer Science and Engineering, Supplement to 2nd edn, ed. by H.F. Mark, N.M. Bikales, C.G. Overberger, Menges G. (Wiley, New York, 1989)

J.M.G. Cowie, E.M. Elexpuru, I.J. McEwen, Polymer 33, 1993 (1992a)

J.M.G. Cowie, M.D. Fernandez, M.J. Fernandez, I.J. McEwen, Polymer 33, 2744 (1992b)

J.M.G. Cowie, G. Li, R. Ferguson, I.J. McEwen, J. Polym. Sci. Polym. Phys. Ed. 30, 1351 (1992c)

J.G. Curro, Polymeric equations of state. J. Macromol. Sci. Rev. Macromol. Chem. C11, 321-366 (1974)

J.G. Curro, Macromolecules 27, 4665 (1994)

J.G. Curro, K.S. Schweizer, Macromolecules 20, 1928 (1987)

J.G. Curro, R.R. Lagasse, R. Simha, J. Appl. Phys. 52, 5892 (1981)

J.G. Curro, R.R. Lagasse, R. Simha, Macromolecules 15, 1621 (1982)

W. De Oliveira, W.G. Glasser, J. Appl. Polym. Sci. 51, 563 (1994)

G.T. Dee, D.J. Walsh, Macromolecules 21, 811, 815 (1988)

M. Dezhu, Z. Ruiyun, L. Xiaolie, Polym. Commun. 36, 3963 (1995)

E.A. DiMarzio, J.H. Gibbs, J. Chem. Phys. 28, 373 (1958)

L. Dinililuc, C. DeKesel, C. David, Eur. Polym. J. 28, 1365 (1992)

D. Dompas, G. Groeninckx, M. Isogawa, T. Hasegawa, M. Kadukura, Polymer 38, 421 (1997)

Z. Dong, J.R. Fried, Comp. Theor. Polym. Sci. 7, 53 (1997)

J. Dong, Y. Ozaki, Macromolecules 30, 286 (1997)

D.C. Douglass, ACS Polym. Prepr. 20(2), 251 (1979)

D.C. Douglass, V.J. McBrierty, Macromolecules 11, 766 (1978)

J. Dudowicz, K.F. Freed, Macromolecules 24, 5076, 5112 (1991)

J. Dudowicz, K.F. Freed, Macromolecules 26, 213 (1993)

J. Dudowicz, K.F. Freed, Macromolecules 28, 6625 (1995) 
J. Dudowicz, K.F. Freed, Macromolecules 29, 7826 (1996a)

J. Dudowicz, K.F. Freed, Macromolecules 29, 8960 (1996b)

J. Dudowicz, M.S. Freed, K.F. Freed, Macromolecules 24, 5096 (1991)

Y. Egawa, S. Imanishi, A. Matsumoto, F. Horii, Polymer 25, 5569 (1996)

H.B. Eitouni, N.P. Balsara, Thermodynamics of polymer blends, Chapter 19, in Physical Properties of Polymers Handbook, ed. by J.E. Mark, 2nd edn. (Springer, New York, 2007)

T.S. Ellis, Polymer 29, 2015 (1988)

T.S. Ellis, Macromolecules 19, 72 (1989)

T.S. Ellis, Polymer 31, 1057 (1990a)

T.S. Ellis, Polym. Eng. Sci. 30, 998 (1990b)

T.S. Ellis, Polymer 33, 1469 (1992)

T.S. Ellis, Polymer 36, 3919 (1995)

T.S. Ellis, Polymer 38, 3837 (1997)

A. Etxeberria, A. Unanue, C. Uriarte, J.J. Iruin, Polymer 38, 4085 (1997)

H. Eyring, M.S. Jhon, Significant Liquid Structures (Wiley, New York, 1969)

S.R. Fahrenholtz, Macromolecules 15, 937 (1982)

A.C. Fernandes, J.W. Barlow, D.R. Paul, J. Appl. Polym. Sci. 32, 5357 (1986)

M.D. Fernandez, M.J. Fernandez, I.J. McEwen, Polymer 38, 2767 (1997)

P.J. Flory, J. Chem. Phys. 9, 660 (1941)

P.J. Flory, Principles of Polymer Chemistry (Cornell University Press, Ithaca, 1953)

P.J. Flory, Dicus. Faraday Soc. 49, 7 (1970)

P.J. Flory, R.A. Orwoll, A. Vrij, J. Am. Chem. Soc. 86, 3507 (1964)

B.H. Flowers, E. Mendoza, Properties of Matter (Wiley, London, 1970)

K.F. Freed, J. Dudowicz, Trends. Polym. Sci. 3, 248 (1995)

K.F. Freed, J. Dudowicz, Macromolecules 29, 625 (1996a)

K.F. Freed, J. Dudowicz, Macromol. Symp. 112, 17 (1996b)

K.F. Freed, J. Dudowicz, Adv. Polym. Sci. 183, 63 (2005)

C. Friedrich, C. Schwarzwlder, R.-E. Riemann, Polymer 37, 2499 (1996)

K.C. Frisch, D. Klempner, H.L. Frish, Polym. Eng. Sci. 22, 1143 (1982)

K.P. Gallagher, X. Zhang, J.P. Runt, G. Hyunh-ba, J.S. Lin, Macromolecules 26, 588 (1993)

P.P. Gan, D.R. Paul, J. Appl. Polym. Sci. 54, 317 (1994a)

P.P. Gan, D.R. Paul, Polymer 35, 3513 (1994b)

P.P. Gan, D.R. Paul, J. Polym. Sci. Part B Polym. Phys. 33, 1693 (1995)

P.P. Gan, D.R. Paul, A.R. Padwa, Polymer 35, 1487, 3351 (1994)

D. Garcia, in Current Topics in Polymer Science, ed. by R.M. Ottenbrite, L.A. Utracki, S. Inoue (Hanser, Munich, 1987)

A. Garton, Polym. Eng. Sci. 23, 663 (1983)

A. Garton, Polym. Eng. Sci. 24, 112 (1984)

R. Gendron, J. Tatiboueet, J. Guevremont, M.M. Dumoulin, L. Piche, Polym. Eng. Sci. 35, 79 (1995)

S.H. Goh, K.S. Siow, Polym. Bull. 20, 393 (1988)

S.H. Goh, D.R. Paul, J.W. Barlow, Polym. Eng. Sci. 22, 34 (1982)

A.A. Goodwin, G.P. Simon, Polymer 37, 991 (1996)

W.W. Graessley, R. Krishnamoorti, N.P. Balsara, L.J. Fetters, D.N. Schulz, J.A. Sissano, Macromolecules 26, 1137 (1993)

W.W. Graessley, R. Krishnamoorti, N.P. Balsara, L.J. Fetters, D.J. Lohse, D.N. Schulz, J.A. Sissano, Macromolecules 27, 2574 (1994a)

W.W. Graessley, R. Krishnamoorti, N.P. Balsara, A. Butera, L.J. Fetters, D.J. Lohse, D.N. Schulz, J.A. Sissano, Macromolecules 27, 3896 (1994b)

W.W. Graessley, R. Krishnamoorti, G.C. Reichart, N.P. Balsara, L.J. Fetters, D.J. Lohse, Macromolecules 28, 1260 (1995)

E.A. Grulke, in Polymer Handbook, ed. by J. Brandrup, E.H. Immergut, 3rd edn. (Wiley, New York, 1989) 
E.A. Guggenheim, Proc. R. Soc. Lond. A 183, 203 (1944)

E.A. Guggenheim, Mixtures (Oxford University Press, Oxford, 1952)

M.C. Gupta, Statistical Thermodynamics (Wiley, New York, 1990)

G. Hadziioannou, R.S. Stein, J. Polym. Sci. B Polym. Phys. 21, 159 (1983)

G. Hadziioannou, R.S. Stein, Macromolecules 17, 567 (1984)

B.R. Hahn, O. Herrmann-Schnherr, J.H. Wendorff, Polymer 28, 201 (1987)

B. Hammouda, J. Macromol. Sci. Part C Polym. Rev. 50, 14 (2010)

B. Hammouda, B.J. Bauer, Macromolecules 28, 4505 (1995)

C.C. Han, J.W. Baurer, J.C. Clark, Y. Muroga, Y. Matsushita, M. Okada, Q. Tran-Cong, T. Chang, I. Sanchez, Polymer 29, 2002 (1988)

C.M. Hansen, J. Paint Technol. 39, 104 (1967)

C.M. Hansen, Europ. Coat. J. 5/94, 305 (1994)

C.M. Hansen, Paint Testing Manual (ASTM, Philadelphia, 1995)

C.M. Hansen, Hansen Solubility Parameters: A Users Handbook (CRC Press, Boca Ratton, 2000)

C. M. Hansen, A. Beerbower, Encyclopaedia of Chemical Technology, A. Standen (eds.), Suppl vol 2 (Interscience, New York, 1971)

J.E. Harris, L.M. Robeson, J. Polym. Sci. Part B Polym. Phys. 25, 311 (1987)

B. Hartmann, M.A. Haque, J. Appl. Polym. Sci. 30, 1553 (1985)

M. Hasegawa, S. Sakurai, M. Takenaka, T. Hashimoto, C.C. Han, Macromolecules 24, 1813 (1991)

T. Hashimoto, in Current Topics in Polymer Science, ed. by R. Ottenbrite, L.A. Utracki, S. Inoue (Carl Hanser, Munich, 1987)

T. Hashimoto, Phase Trans. 12, 47 (1988)

T. Hashimoto, J. Kumaki, H. Kawai, Macromolecules 16, 641 (1983)

T. Hashimoto, T. Takebe, S. Suehiro, Polym. J. 18, 123 (1986)

Y. He, B. Zhu, Y. Inoue, Prog. Polym. Sci. 29, 10211051 (2004)

E. Helfand, Macromolecules 8, 552 (1975a)

E. Helfand, J. Chem. Phys. 62, 999 (1975b)

E. Helfand, J. Chem. Phys. 63, 2192 (1975c)

E. Helfand, A. Sapse, J. Chem. Phys. 62, 1327 (1975)

E. Helfand, Y. Tagami, Polym. Lett. 9, 741 (1971a)

E. Helfand, Y. Tagami, J. Chem. Phys. 57, 1812 (1971b)

E. Helfand, Y. Tagami, J. Chem. Phys. 56, 3592 (1972)

C. Herkt-Maetzky, J. Schelten, Phys. Rev. Lett. 51, 896 (1983)

W.M. Hess, C.R. Herd, P.C. Vegvari, Rubber Chem. Technol. 66, 329 (1993)

N. Higashida, J. Kressler, T. Inoue, Polymer 36, 2761 (1995)

H. Higuchi, Z. Yu, A.M. Jamieson, R. Simha, J.D. McGgervey, J. Polym. Sci.: Polym. Phys. 33, 2295 (1995)

J. Hildebrand, R.L. Scott, The Solubility of Nonelectrolytes, 3rd edn. (Reinhold, New York, 1950)

J. Hildebrand, R.L. Scott, Regular Solutions (Prentice-Hall, Englewood Cliffs, 1962)

J.D. Honeycutt, SPE Tech. Pap. 38, 1602 (1992a)

J.D. Honeycutt, ACS Polym. Prepr. 33, 529 (1992b)

B.K. Hong, J.Y. Kim, W.H. Jo, S.C. Lee, Polymer 38, 4373 (1997)

K.G. Honnell, J.G. Curro, K.S. Schweizer, Macromolecules 23, 3496 (1990)

K.G. Honnell, J.D. McCoy, J.G. Curro, K.S. Schweizer, A.H. Narten, A. Habenschuss, J. Chem. Phys. 94, 4659 (1991)

S.-S. Hou, Q. Chen, K. Schmidt-Rohr, Macromolecules 37, 1999 (2004)

K.L. Hoy, J. Paint Technol. 42, 76 (1970)

C.C. Hsu, P.H. Geil, Polym. Eng. Sci. 27, 1542 (1987)

Z.H. Huang, L.H. Wang, Makromol. Chem. Rapid Commun. 7(255) (1986)

M.L. Huggins, J. Chem. Phys. 9, 440 (1941)

P.P. Huo, P. Cebe, Macromolecules 26, 3127 (1993)

K. Ikawa, S. Hosoda, Polym. Netw. Blends 1, 102 (1991) 
H. Inagaki, H. Suzuki, M. Kurata, J. Polym. Sci. C15, 409 (1966)

T. Inoue, T. Ougizawa, O. Yasuda, K. Miyasaka, Polym. Commun. 18, 57 (1985)

M. Iriarte, J.I. Iribarren, A. Etxeberria, J.J. Iruin, Polymer 30, 1160 (1989)

T. Izumitani, T. Hashimoto, J. Chem. Phys. 83, 3694 (1985)

H. Jagger, E.J. Vorenkamp, G. Challa, Polym. Commun. 24, 290 (1983)

R.K. Jain, R. Simha, Macromolecules 13, 1501 (1980)

R.K. Jain, R. Simha, Macromolecules 17, 2663 (1984)

R.K. Jain, R. Simha, P. Zoller, J. Polym. Sci. Polym. Phys. Ed. 20, 1399 (1982)

Y. Jinghua, C. Xue, G.C. Alfonso, A. Turturro, E. Pedemonte, Polymer 38, 2127 (1997)

F. Joanny, L. Leibler, J. Phys. (Paris) 39, 951 (1978)

H.Y. Jung, Polym. J. 28, 1048 (1996)

W.G. Jung, E.W. Fischer, Makromol. Chem. Macromol. Symp. 16, 281 (1988)

R.P. Kambour, R.C. Bopp, A. Maconnachie, W.J. MacKnight, Polym. Commun. 21, 133 (1980)

H.W. Kammer, C. Kumerlowe, Polym. Eng. Sci. 36, 1608 (1996)

D.S. Kaplan, J. Appl. Polym. Sci. 20, 2615 (1976)

S. Kaplan, ACS Polym. Prepr. 25(1), 356 (1984)

J. Karger-Kocsis, L. Kiss, Polym. Eng. Sci. 27, 254 (1987)

A. Karim, K. Yurekli, J.C. Meredith, E.J. Amis, R. Krishnamoorti, Polym. Eng. Sci. 42, 1836 (2002)

C.K. Kim, D.R. Paul, Polymer 33, 1630, 2089 (1992)

J.H. Kim, J.W. Barlow, D.R. Paul, J. Polym. Sci. Part B Polym. Phys. Ed. 27, 223 (1989)

C.H. Kim, J.-K. Park, T.-S. Hwang, Polym. Eng. Sci. 36, 535 (1996)

A.M. Kisselev, E. Manias, Fluid Phase Equilibria 261, 69 (2007)

L.A. Kleintjens, Fluid Phase Equilibria 10, 183 (1983)

V.J. Klenin, Stability and Phase Separation (Elsevier, Amsterdam, 1999)

W. Klopffer, Introduction to Polymer Spectroscopy (Springer, Berlin, 1984)

J.L. Koenig, M.J.M. Tovar-Rodriguez, Appl. Spectrosc. 35, 543 (1981)

R. Koningsveld, Ph.D. thesis, University Leiden (1967)

R. Koningsveld, L.A. Kleintjens, H.M. Schoffaleers, Pure Appl. Chem. 39, 1 (1974)

R. Koningsveld, L.A. Kleintjens, A.M. Leblans-Vinck, J. Phys. Chem. 91, 6423 (1987)

R. Koningsveld, W.H. Stockmayer, E. Nies, Polymer Phase Diagrams: A Textbook (Oxford University Press, New York, 2001)

R. Krishnamoorti, W.W. Graessley, N.P. Balsara, D.J. Lohse, J. Chem. Phys. 100, 3894 (1994a)

R. Krishnamoorti, W.W. Graessley, N.P. Balsara, D.J. Lohse, Macromolecules 27, 3073 (1994b)

R. Krishnamoorti, W.W. Graessley, L.J. Fetters, R.T. Garner, D.J. Lohse, Macro-molecules 28, 1252 (1995)

R. Krishnamoorti, W.W. Graessley, G.T. Dee, D.J. Walsh, L.J. Fetters, D.J. Lohse, Macromolecules 29, 367 (1996)

P.P. Kundu, D.K. Tripathy, S. Banners, Polymer 37, 2423 (1996)

S.L. Kwolek, P.W.,Morgan, W.R. Sorenson, U.S. Patent 3,063,966, 13 Nov 1962, Appl. 5 Feb 1958, to E. I. du Pont de Nemours \& Company

M. Kyotani, H. Kanetsuna, J. Macromol. Sci. Phys. B26, 325 (1987)

T. Kyu, J.M. Saldanha, M.K. Kiesel, in Two-Phase Polymer Systems, ed. by L.A. Utracki (Hanser Publications, Munich, 1991)

D. Lath, J.M.G. Cowie, Makromol. Chem. Macromol. Symp. 16, 103 (1988)

A.A. Lefebvre, J.H. Lee, N.V. Balsara, B. Hommouda, Macromolecules 33, 7977 (2000)

A.A. Lefebvre, J.H. Lee, N.V. Balsara, B. Hommouda, Macromolecules 35, 7758 (2002)

E.G. Lezcano, C.S. Coll, M.G. Prolongo, Polymer 37, 3603 (1996)

T.S. Lin, Ph.D. thesis, Virginia Polytechnic Institute (1983)

C.C. Lin, S.V. Jonnalagadda, N.P. Balsara, C.C. Han, R. Krishnamoorti, Macromolecules 29, 661 (1996)

D. Lohse, Rubber Chem. Technol. 67, 367 (1994)

J.D. Londono, G.D. Wignall, Macromolecules 30, 3821 (1997) 
J.D. Londono, A.H. Narten, G.D. Wignall, K.G. Honnell, E.T. Hsieh, T.W. Johnson, F.S. Bates, Macromolecules 27, 2864 (1994)

X. Lu, R.A. Weiss, Macromolecules 24, 4381 (1991)

X. Lu, R.A. Weiss, Macromolecules 25, 6185 (1992)

A. Luciani, M.F. Champagne, L.A. Utracki, Polym. Netw. Blends 6, 41 (1996a)

A. Luciani, M.F. Champagne, L.A. Utracki, Polym. Netw. Blends 6, 51 (1996b)

A. Luciani, M.F. Champagne, L.A. Utracki, J. Polym. Sci. B Polym. Phys. 35, 1393 (1997)

A. Maconnachi, J. Kressler, B. Rudolf, P. Reichert, F. Koopmann, H. Frey, R. Mülhaupt, Macromolecules 29, 1490 (1996)

R.-D. Maier, R.P. Kambour, D.M. White, S. Rostami, D.J. Walsh, Macromolecules 17, 2645 (1984)

R.-D. Maier, J. Kressler, B. Rudolf, P. Reichert, F. Koopmann, H. Frey, R. Mülhaupt, Macromolecules 29, 1490 (1996)

P. Maiti, A.K. Nandi, Macromolecules 28, 8511 (1995)

W.M. Marr, Macromolecules 28, 8470 (1995)

E. Martuscelli, G. Demma, E. Rossi, A.L. Segre, Polym. Commun. 6, 125 (1983)

E. Martuscelli, M. Pracella, W.P. Yue, Polymer 25, 1097 (1984)

S. Matsuda, Polym. J. 23, 435 (1991)

V.J. McBrierty, K.J. Packer, Nuclear Magnetic Resonance in Solid Polymers (Cambridge University Press, Cambridge, 1993)

V.J. McBrierty, D.C. Douglass, T.K. Kwei, Macromolecules 11, 1265 (1978)

J.E. McKinney, R. Simha, Macromolecules 7, 894 (1974)

L.P. McMaster, Macromolecule 6, 760 (1973)

L.P. McMaster, ACS Adv. Chem. Ser. 142, 43 (1975)

L.P. McMaster, O. Olabisi, ACS Org. Coat. Plast. Chem. Prepr. 35, 322 (1975)

J.C. Meredith, A. Karim, E.J. Amis, Macromolecules 33, 5760 (2000)

J.C. Meredith, A. Karim, E.J. Amis, MRS Bull. 27, 330 (2002)

A. Molnar, A. Eisenberg, Macromolecules 25, 5774 (1992)

B.S. Morra, R.S. Stein, Polym. Eng. Sci. 24, 311 (1984)

E.J. Moskala, Ph.D. thesis, Pennsylvania State University (1984)

B. Mujumdar, H. Keskkula, D.R. Paul, N.G. Harvey, Polymer 35, 4263 (1994a)

B. Mujumdar, H. Keskkula, D.R. Paul, Polymer 35, 5453, 5468 (1994b)

A.K. Mukherji, M.A. Butler, D.L. Evans, J. Appl. Polym. Sci. 25, 1145 (1980)

E.C. Muniz, P.A.M. Vasquez, R.E. Bruns, S.P. Nunes, B.A. Wolf, Makromol. Chem. Rapid Commun. 13, 45 (1992)

C.T. Murray, J.W. Gilmer, R.S. Stein, Macromolecules 18, 996 (1985)

W.L. Nachlis, J.T. Bendler, R.P. Kambour, W.J. MacKnight, Macromolecules 28, 7869 (1995)

M.K. Neo, S.H. Goh, Polymer 33, 3203 (1992)

E. Nies, A. Stroeks, R. Simha, R.K. Jain, Colloid Polym. Sci. 268, 731 (1990)

T. Nishi, T.K. Kwei, Polymer 16, 285 (1975)

T. Nishi, T.T. Wang, Macromolecules 8, 909 (1975)

T. Nishi, T.T. Wang, Macromolecules 10, 421 (1977)

T. Nishi, T.T. Wang, T.K. Kwei, Macromolecules 8, 227 (1975)

M. Nishimoto, Y. Takami, A. Tohara, H. Kasahara, Polymer 36, 1441 (1995)

S. Nojima, K. Tsutsumi, T. Nose, Polym. J. 14, 225, 289, 907 (1982)

E. Ogawa, N. Yamaguchi, M. Shima, Polym. J. 18, 903 (1986)

B. Ohlsson, B. Tørnell, Polym. Eng. Sci. 36, 1547 (1996)

T. Ohnaga, T. Sato, S. Nagata, Polymer 38, 1073 (1997)

O. Olabisi, R. Simha, J. Appl. Polym. Sci. 21, 149 (1977)

O. Olabisi, L.M. Robeson, M.T. Shaw, Polymer-Polymer Miscibility (Academic, New York, 1979)

T. Ougizawa, T. Inoue, Polym. J. 18, 521 (1986)

T. Ougizawa, T. Inoue, H.W. Kammer, Macromolecules 18, 2089 (1985)

T. Ougizawa, G.T. Dee, D.J. Walsh, Macromolecules 24, 3834 (1991) 
P.C. Painter, M.M. Coleman, Hydrogen bonding systems, Chapter 4, in Polymer Blends, ed. by

D.R. Paul, C.B. Bucknall, vol. 1 (Wiley, New York, 2000)

P.C. Painter, Y. Park, M.M. Coleman, Macromolecules 21, 66 (1988)

P.C. Painter, Y. Park, M.M. Coleman, Macromolecules 22, 570 (1989a)

P.C. Painter, Y. Park, M.M. Coleman, Macromolecules 22, 580 (1989b)

P.C. Painter, J. Graf, M.M. Coleman, J. Chem. Phys. 92, 6166 (1990)

C.P. Papadopoulou, N.K. Kalfoglu, Polymer 38, 631 (1997)

D.D. Patterson, Polym. Eng. Sci. 22, 64 (1982)

D.R. Paul, J.W. Barlow, Polymer 25, 487 (1984)

D.R. Paul, C.B. Bucknall (eds.), Polymer Blends: Vol. 1: Formulation and Vol. 2: Performance (Wiley, New York, 2000)

G.J. Pehlert, P.C. Painter, B. Veytsman, M.M. Coleman, Macromolecules 30, 3671 (1997)

J. Peng, S.H. Goh, S.Y. Lee, K.S. Siow, Polym. Netw. Blends 4, 139 (1994)

J. Pennacchia, Ph.D. thesis, Polytechnic Institute, New York (1986)

J.P. Pennings, St. R. Manley, Macromolecules 29, 77 (1996)

M. Piau, C. Verdier, Ultrason. Int. 93, 423 (1993)

L.Z. Pillon, L.A. Utracki, Polym. Proc. Eng. 4, 375 (1986)

D.N. Pinder, Macromolecules 30, 226 (1997)

J. Plans, W.J. MacKnight, F.E. Karasz, Macromolecules 17, 810 (1984)

I. Prigogine, N. Trappeniers, V. Mathot, Disc. Faraday Sci. 15, 93 (1953)

I. Prigogine, A. Bellemans, V. Mathot, The Molecular Theory of Solutions (North-Holland, Amsterdam, 1957)

R.E. Prud'homme, Polym. Eng. Sci. 22, 1138 (1982)

M. Puma, Polym. Adv. Technol. 8, 39 (1997)

C. Qian, S.J. Mumby, B.E. Eichinger, Macromolecules 24, 1655 (1991)

H.-J. Radusch, N.T. Tung, C. Wohlfarth, Angew. Makromol. Chem. 235, 175 (1996)

D. Rana, B.M. Mandal, S.N. Bhattacharyya, Polymer 37, 2439 (1996)

L. Ratke, W.K. Thieringer, Acta Metal. 33, 1793 (1985)

S. Reich, Phys. Lett. 114A, 90 (1986)

G.C. Reichart, W.W. Graessley, R.A. Register, R. Krishnamoorti, D.J. Lohse, Macromolecules 30, 3363 (1997)

A.R. Rennie, Characterization of Solid Polymers, S.J. Spells (eds.), (Chapman \& Hall, London, 1992)

L.M. Robeson, in Polymer Compatibility and Incompatibility, ed. by K. Solc (Harwood Academy, New York, 1980)

P.A. Rodgers, J. Appl. Polym. Sci. 48, 1061 (1993a)

P.A. Rodgers, J. Appl. Polym. Sci. 50, 2075 (1993b)

J.L. Rodriguez, J.I. Eguizabal, J. Nazabal, Polym. J. 28, 501 (1996)

J.M. Rodriguez-Parada, V. Percec, J. Polym. Sci. Chem. Ed. 24, 579 (1986)

R.J. Roe, J. Chem. Phys. 62, 490 (1975)

J. Roovers, P.M. Toporowski, Macromolecules 25, 3454 (1992)

B. Rudolf, J. Kressler, K. Shimomami, T. Ougizawa, T. Inoue, Acta Polym. 46, 312 (1995)

J. Runt, K.P. Gallagher, Polym. Comm. 32, 180 (1991)

H. Saito, Y. Fujita, T. Inoue, Polym. J. 19, 405 (1987)

I.C. Sanchez, Polymer 30, 471 (1989)

I.C. Sanchez, J. Cho, Polymer 36, 2929 (1995)

I.C. Sanchez, R.H. Lacombe, J. Phys. Chem. 80, 2352 (1976)

I.C. Sanchez, R.H. Lacombe, J. Polym. Soc. Polym. Lett. Ed. 15, 71 (1977)

I.C. Sanchez, R.H. Lacombe, Macromolecules 11, 1145 (1978)

I.C. Sanchez, J. Cho, W.-J. Chen, Macromolecules 26, 4234 (1993)

S.I. Sandler, H.S. Wu, Ind. Eng. Chem. Res. 30, 881, 889 (1991)

M. Sankarapandian, K. Kishore, Polymer 37, 2957 (1996)

T. Sato, C.C. Han, J. Chem. Phys. 88, 2057 (1988) 
T. Sato, M. Endo, T. Shiomi, K. Imai, Polymer 37, 2131 (1996a)

T. Sato, M. Tohyama, M. Suzuki, T. Shiomi, K. Imai, Macromolecules 29, 8231 (1996b)

T. Sato, M. Suzuki, M. Tohyama, M. Endo, T. Shiomi, K. Imai, Polym. J. 29, 417 (1997)

B.B. Sauer, B.S. Hsiao, K.L. Faron, Polymer 37, 445 (1996)

C. Schipp, M.J. Hill, P.J. Barham, V.M. Clocke, J.S. Higgins, L. Oiarzabal, Polymer 37, 2291 (1996)

B. Schlund, L.A. Utracki, Polym. Eng. Sci. 27, 1523 (1987a)

B. Schlund, L.A. Utracki, Polym. Eng. Sci. 27, 359, 380 (1987b)

K. Schmidt-Rohr, J. Clauss, B. Blmich, H.W. Spiess, Magn. Reson. Chem. 28, S3-S9 (1990)

K. Schulze, J. Kressler, H.W. Kammer, Polymer 34, 3704 (1993)

D. Schwahn, Adv. Polym. Sci. 183, 1 (2005)

D. Schwahn, K. Mortensen, T. Springer, H. Yee-Madeira, R. Thomas, J. Chem. Phys. 87, 6078 (1987)

K.S. Schweizer, Macromolecules 26, 6050 (1993)

K.S. Schweizer, J.G. Curro, J. Chem. Phys. 91, 5059 (1989)

M. Seki, H. Uchida, Y. Maeda, S. Yamauchi, K. Takagi, Y. Ukai, Y. Matsushita, Macromolecules 33, $9712(2000)$

C.J. Serman, Y. Xu, J. Graf, P.C. Painter, M.M. Coleman, Macromolecules 22, 2019 (1989)

C.J. Serman, Y. Xu, J. Graf, P.C. Painter, M.M. Coleman, Polymer 32, 516 (1991)

V.S. Shah, J.D. Keitz, D.R. Paul, J.W. Barlow, J. Appl. Polym. Sci. 32, 3863 (1986)

C.K. Sham, D.J. Walsh, Polymer 28, 804 (1987)

M.T. Shaw, J. Appl. Polym. Sci. 18, 449 (1974)

M. Shibayama, K. Uenoyama, J.-I. Oura, S. Nomura, T. Iwamoto, Polymer 36, 4811 (1995)

K. Shinoda, Principles of Solutions and Solubility (Marcel Dekker, New York, 1978)

T. Shiomi, F.E. Karasz, W.J. MacKnight, Macromolecules 19, 2274 (1986)

G.O. Shonaike, G.P. Simon (eds.), Polymer Blends and Alloys (Marcel-Dekker, New York, 1999)

A.R. Shultz, A.L. Young, Macromolecules 13, 663 (1980)

A. Silberberg, W. Kuhn, Nature 170, 450 (1952)

A. Silberberg, W. Kuhn, J. Polym. Sci. 13, 21 (1954)

R. Simha, T. Somcynsky, Macromolecules 2, 342 (1969)

R. Simha, C.E. Weil, J. Macromol. Sci. Phys. B4, 215 (1970)

R. Simha, P.S. Wilson, Macromolecules 6, 908 (1973)

R. Simha, U. Yahsi, Statistical thermodynamics of hydrocarbon fluids. J. Chem. Soc. Faraday Trans. 91, 2443 (1995)

K. Solc (ed.), Polymer Compatibility and Incompatibility (Harwood Academic Publishers, New York, 1982)

R.S. Spencer, G.D. Gilmore, J. Appl. Phys. 20, 504 (1949)

E.O. Stejskal, J. Schaefer, M.D. Sefcik, R.A. McKay, Macromolecules 14, 2683 (1981)

R. Steller, D. Zuchowska, J. Appl. Polym. Sci. 41, 1595 (1990)

G.R. Strobl, J.T. Bendler, R.P. Kambour, A.R. Schulz, Macromolecules 19, 2683 (1986)

A. Stroeks, E. Nies, Macromolecules 23, 4092 (1990)

P.G. Tait, Phys. Chem. 3, 1 (1888)

M. Takahashi, S. Kinoshita, T. Nose, Polym. Prepr. Jap. 34, 2421 (1985)

M. Takahashi, H. Hirouchi, S. Kinoshita, T. Nose, J. Phys. Soc. Jap. 55, 2687 (1986)

H. Takahashi, Y. Inoue, O. Kamigaito, K. Osaki, Kobunshi Ronbunshu 47, 7, 611 (1990)

K. Takakuwa, S. Gupta, D.R. Paul, J. Polym. Sci. Part B Polym. Phys. 32, 1719 (1994)

M. Takenaka, K. Tanaka, T. Hashimoto, in Contemporary Topics in Polymer Science, ed. by W.M. Culberston, vol. 6 (Plenum Press, New York, 1989)

H. Takeno, S. Koizumi, H. Hasegawa, T. Hashimoto, Macromolecules 29, 2440 (1996)

G. ten Brinke, F.E. Karasz, Macromolecules 17, 815 (1984)

G. ten Brinke, F.E. Karasz, W.J. MacKnight, Macromolecules 16, 1827 (1983)

S.-P. Ting, Ph.D. thesis, Polytechnic Institute, New York (1980)

H. Tompa, Polymer Solutions (Butterwords Scientific Publications, London, 1956) 
L.A. Utracki, J. Appl. Polym. Sci. 6, 399 (1962)

L.A. Utracki, Polym. J. 3, 551 (1972)

L.A. Utracki, Polym. Eng. Sci. 25, 655 (1985)

L.A. Utracki, J. Rheol. 30, 829 (1986)

L.A. Utracki, Polymer Alloys and Blends (Hanser, Munich, 1989)

L.A. Utracki, J. Rheol. 35, 1615-1637 (1991)

L.A. Utracki, in Rheological Fundamentals of Polymer Processing, ed. by J.A. Covas, J.F. Agassant, A.C. Diogo, J. Vlachopoulos, K. Walters (Kluver Academic, Dordrecht, 1995)

L.A. Utracki, B. Schlund, Polym. Eng. Sci. 27, 367 (1987a)

L.A. Utracki, B. Schlund, Polym. Eng. Sci. 27, 1512 (1987b)

L.A. Utracki, R. Simha, U. Yahsi, Interrelationships between P-V-T and flow behavior of hydrocarbons. in Proceedings of the XII International Congress on Rheology, Quebec, 18-23 Aug 1996

L.A. Utracki, R. Simha, in Free-Volume Application to Foaming, NRCC/IMI Symposium on Polymer Foaming, Boucherville 28, Jan 1997

J.J. van Aarsten, Eur. Polym. J. 6, 919 (1970)

G.O.R.A. van Ekenstein, H. Deuring, G. ten Brinke, T.S. Ellis, Polymer 38, 3025 (1997)

D.W. van Krevelen, Properties of Polymers, 2nd edn. (Elsevier, Amsterdam, 1976)

D.W. van Krevelen, in Computational Modeling of Polymers, ed. by J. Bicerano (Marcel Dekker, New York, 1992)

C.J. van Oss, M.K. Chaudhury, R.J. Good, Chem. Rev. 88, 927 (1988)

C. Verdier, M. Piau, Recent Prog. Genie Procedes, 9(38), (Genie des Procedes dans la Chaine des Polymeres et dans la Chaine Catalytique), 25-30 (1995)

D. Vesely, Polym. Eng. Sci. 36, 1586 (1996)

D. Vesely, D.S. Finch, Makromol. Chem. Macromol. Symp. 16, 329 (1988)

I.G. Voigt-Martin, K.-H. Leister, R. Rosenau, R. Koningsveld, J. Polym. Sci. Part B Polym. Phys. 24, 723 (1986)

J.R. Waldram, The Theory of Thermodynamics (Cambridge University Press, Cambridge, 1985)

D.J. Walsh, S. Rostami, Macromolecules 18, 216 (1985)

D.J. Walsh, P. Zoller, Makromol. Chem. 188, 2193 (1987)

D.J. Walsh, J.S. Higgins, C. Zhikuan, Polymer 22, 1005 (1982)

L.H. Wang, R.S. Porter, J. Polym. Sci. Polym. Phys. Ed. 21, 1815 (1983)

T.C. Ward, T.S. Lin, ACS Adv. Chem. Ser. 206, 59 (1984)

R.W. Warfield, B. Hartmann, Polymer 21, 31 (1980)

G. Webb (ed.), Modern Magnetic Resonance (Springer, Dordrecht, 2006)

J.L. White, M. Wachowicz, Polymer blend miscibility - NMR, Chapter 7. in Annual Reports on NMR Spectroscopy, vol. 64 (Academic Press, Elsevier, London, 2008)

C. Wohlfarth, CRC Handbook of Thermodynamic Data of Aqueous Polymer Solutions (CRC Press, Boca Raton, 2004)

C. Wohlfarth, CRC Handbook of Liquid-Liquid Equilibrium Data of Polymer Solutions (Taylor \& Francis, Boca Raton, 2008)

B.A. Wolf, Makromol. Chem. Rapid Commun. 189, 1613 (1980)

B.A. Wolf, Macromolecules 17, 615 (1984)

E.M. Woo, J.W. Barlow, D.R. Paul, J. Appl. Polym. Sci. 28, 1347 (1983)

E.M. Woo, J.W. Barlow, D.R. Paul, J. Appl. Polym. Sci. 29, 3837 (1984)

E.M. Woo, J.W. Barlow, D.R. Paul, Polymer 26, 763 (1985)

E.M. Woo, J.W. Barlow, D.R. Paul, J. Appl. Polym. Sci. 32, 3889 (1986)

H.-K. Xie, E. Nies, A. Stroeks, R. Simha, Polym. Eng. Sci. 32, 1654 (1992)

H.-K. Xie, R. Simha, P. Moulin, in FoamTech Meeting, NRCC/IMI, Boucherville, 12 Sept 1997

Y. Xu, J. Graf, P.C. Painter, M.M. Coleman, Polymer 32, 3103 (1991)

U. Yahsi, Ph.D. thesis, Case Western Reserve University, Department of Physics, Cleveland (1994)

H. Yamakawa, Modern Theory of Polymer Solutions (Harper \& Row, New York, 1971) 
H. Yang, G. Hadziioannou, R.S. Stein, J. Polym. Sci. B: Polym. Phys. 21, 159 (1983)

K. Yurekli, A. Karim, E.J. Amis, R. Krishnamoorti, Macromolecules 37, 507 (2004)

S. Zhang, R. Prud'homme, J. Polym. Sci. Part B Polym. Phys. 24, 723 (1987)

S. Zheng, J. Huang, Y. Li, Q. Guo, J. Polym. Sci. Part B Polym. Phys. 35, 1383 (1997)

S. Ziaee, D.R. Paul, J. Polym. Sci. Part B Polym. Phys. 34(1996), 2641 (1996)

S. Ziaee, D.R. Paul, J. Polym. Sci. Part B Polym. Phys. 35, 489, 831 (1997)

P. Zoller, in Polymer Handbook, ed. by J. Brandrup, E.H. Immergut, 3rd edn. (Wiley, New York, 1989)

P. Zoller, H.H. Hoehn, J. Polym. Sci. Polym. Phys. Ed. 20, 1385 (1982)

P. Zoller, D. Walsh, Standard Pressure-Volume-Temperature Data for Polymers (Technomic Publishing Company, Lancaster-Basel, 1995) 